\title{
Competition between parallel sensorimotor learning systems
}

Scott T. Albert ${ }^{1,7}$, Jihoon Jang ${ }^{1}$, Shanaathanan Modchalingam², Marius 't Hart', Denise Henriques' Gonzalo Lerner ${ }^{3}$, Valeria Della-Maggiore ${ }^{3}$, Adrian M. Haith ${ }^{4}$, John W. Krakauer ${ }^{4,5,6}$, and Reza Shadmehr ${ }^{1}$

1. Department of Biomedical Engineering, Johns Hopkins School of Medicine, Baltimore MD

2. Department of Kinesiology and Health Science, York University, Toronto, Canada

3. Deparamento de Fisiología y Biofísia, Universidad de Buenos Aires, Buenos Aires, Argentina

4. Department of Neurology, Johns Hopkins School of Medicine, Baltimore MD

5. Department of Neuroscience, Johns Hopkins School of Medicine, Baltimore MD

6. The Santa Fe Institute, Santa Fe, NM

7. University of North Carolina, Chapel Hill, NC

Correspondence: Scott Albert, 7302 Mary Ellen Jones, University of North Carolina at Chapel Hill School of Medicine, Chapel Hill, NC 27599, USA. Email: scottalbert1@gmail.com.

Acknowledgements: This work was supported by grants from the National Institutes of Health (R01NS078311, F32NS095706), and the National Science Foundation (CNS-1714623). 


\section{Abstract}

Sensorimotor learning is supported by at least two parallel systems: a strategic process that benefits from explicit knowledge, and an implicit process that adapts subconsciously. How do these systems interact? Does one system's contributions suppress the other, or do they operate independently? Here we illustrate that during reaching, implicit and explicit systems both learn from visual target errors. This shared error leads to competition such that an increase in the explicit system's response siphons away resources that are needed for implicit adaptation, thus reducing its learning. As a result, steady-state implicit learning can vary across experimental conditions, due to changes in strategy. Furthermore, strategies can mask changes in implicit learning properties, such as its error sensitivity. These ideas, however, become more complex in conditions where subjects adapt using multiple visual landmarks, a situation which introduces learning from sensory prediction errors in addition to target errors. These two types of implicit errors can oppose each other, leading to another type of competition. Thus, during sensorimotor adaptation, implicit and explicit learning systems compete for a common resource: error.

\section{Introduction}

When our movements are perturbed, we become aware of our errors, and through our own strategy, or instructions from a coach, engage an explicit learning system to improve our outcome ${ }^{1,2}$. This awareness, is not required to adapt; our brain also uses an implicit learning system that partially corrects behavior without our conscious awareness ${ }^{1,2}$. How do these two systems interact during sensorimotor adaptation?

Suppose that both systems learn from the same error. In this case, when one system adapts, it will reduce the error that drives learning in the other system; thus, the two parallel systems will compete to "consume" a common error. Alternatively, suppose the two systems learn from separate errors, and each produces an output to minimize its own error. In this case, when one system adapts to its error, it could change behavior in ways that paradoxically increase the other system's error.

Current models suggest that adaptation is driven by two distinct error sources: a task error ${ }^{3-5}$, and a prediction error ${ }^{2,6,7}$. One leading theory suggests that the explicit system acts to decrease errors in task performance, while the implicit system acts to reduce errors in predicting sensory outcomes ${ }^{2,8,9}$. In this model, strategies have no impact on implicit learning. A second theory suggests that task errors can drive learning in both systems ${ }^{3,10-12}$. In this model, implicit and explicit systems will compete with one another.

Suppose implicit and explicit systems share at least one common error source. What will happen when experimental conditions enhance one's explicit strategy? In this case, increases in explicit strategy will siphon away the error that the implicit system needs to adapt, thus reducing total implicit learning without directly changing implicit learning properties (e.g., its memory retention or sensitivity to error). This reduction in implicit learning creates the illusion that the implicit system was directly altered by the experimental manipulation, when in truth, it was only responding to changes in strategy.

Competitive interactions like this highlight the need to distinguish between an adaptive system's learning properties such as its sensitivity to an error, and its learning timecourse, i.e., the contribution it makes to overall adaptation at any point in time. In a competitive system, an adaptive processes' learning timecourse depends not only on its own learning properties, but also its competitors' learning properties. In cases where implicit and explicit systems share an error source, one system's behavior can 
be shaped not only by its past experience, but also by changes in the other system. Thus, competition may play an important role in savings $s^{13-17}$ and interference paradigms ${ }^{18-20}$ where learning properties change over time. Measuring the interdependence between implicit and explicit learning may help to explain the disconnect between studies that have suggested acceleration in motor learning is subserved solely by explicit strategy ${ }^{13,21-24}$, and studies that have pointed to concomitant changes in implicit learning systems $\mathrm{s}^{3,25,26}$.

Here, we begin by mathematically ${ }^{11,12,27-29}$ considering the extent to which implicit and explicit systems are engaged by task errors and prediction errors. The hypotheses make diverging predictions, which we test in various contexts. Our work suggests that in some contexts ${ }^{2,8}$, prediction errors and task errors both make important contributions to implicit learning (Results Part 3). In other contexts, the data suggest that the implicit system is primarily driven by task errors shared with the explicit system (Results Part 1). In this latter case, the competition theory explains why increases ${ }^{30,31}$ or decreases ${ }^{32,33}$ in explicit strategy cause an opposite change in implicit learning. This model explains why in some cases implicit adaptation can saturate as perturbations grow ${ }^{30,34,35}$, but not others ${ }^{35,36}$. The model also explains why participants that utilize large explicit strategies can exhibit less implicit ${ }^{12}$ or procedural learning ${ }^{32}$, than those who do not. Finally, the theory provides an alternate way to interpret implicit contributions to two learning hallmarks: savings ${ }^{13}$ and interference ${ }^{19}$ (Results Part 2).

Altogether, our results illustrate that sensorimotor adaptation is shaped by competition between parallel learning systems, both engaged by task errors.

\section{Results}

In visuomotor rotation paradigms, participants move a cursor that travels along a rotated path (Fig. 1A). This perturbation causes adaptation, resulting in both implicit recalibration (Fig. 1A, implicit) and explicit (intentional) re-aiming (Fig. 1A, aim) $)^{2,8,37,38}$.

Current models suggest that the rotation $r$ creates two distinct error sources. One error source is the deviation between cursor and target: a target error ${ }^{3-5}$. Notably, this target error (Fig. 1A, target error) is altered by both implicit $\left(x_{i}\right)$ and explicit $\left(x_{e}\right)$ adaptation:

$$
e_{\text {target }}^{(n)}=r^{(n)}-\left(x_{i}^{(n)}+x_{e}^{(n)}\right)
$$

In addition, a second error is created due to our expectation that the cursor should move toward where we aimed our movement: a sensory prediction error $(\mathrm{SPE})^{2,6,7}$. SPE is the deviation between the aiming direction (the expected cursor motion) and where we observed the cursor's actual motion (Fig. 1A, sensory prediction error). Critically, because this error is anchored to our aim location, it changes over time in response to implicit adaptation alone:

$$
e_{S P E}^{(n)}=r^{(n)}-x_{i}^{(n)}
$$

How does the implicit learning system respond to these two error sources? State-space models describe implicit adaptation as a process of learning and forgetting ${ }^{11,12,27-29}$ :

$$
x_{i}^{(n+1)}=a_{i} x_{i}^{(n)}+b_{i} e^{(n)}
$$

Forgetting is controlled by a retention factor $\left(a_{i}\right)$ which determines how strongly we retain the adapted state. Learning is controlled by error sensitivity $\left(b_{i}\right)$ which determines the amount we adapt in response to an error (e.g., an SPE or a target error). 
Here we will contrast two possibilities: (1) the implicit system responds primarily to target error, or (2) the implicit system responds primarily to SPE. In a target error learning system, explicit strategy will reduce the target error in Eq. (1). This decrease in target error will lead to a competition between implicit and explicit systems, i.e., increasing explicit strategy reduces target error, which will then decrease implicit learning. Competition in a target error model will occur over the entire learning timecourse and can lead to unintuitive implicit learning phenotypes (Appendix 1.2). While these implicit behaviors can be observed at any point during adaptation, they are easiest to examine during steadystate adaptation (Appendix 1.1).

Consider how Eq. (3) behaves in the steady-state condition. Like adapted behavior ${ }^{10,26,39,40}$, Eq. (3) approaches an asymptote with extended exposure to a rotation. This steady-state (Fig. 1B, implicit) occurs when learning and forgetting counterbalance each other.

Consider a system where target errors alone drive implicit learning. In this system, total (steadystate) implicit learning is determined by Eqs. (1) and (3):

$$
x_{i}^{s s}=\frac{b_{i}}{1-a_{i}+b_{i}}\left(r-x_{e}^{s s}\right)
$$

Eq. (4) demonstrates a competition between implicit and explicit systems; the total amount of implicit adaptation $\left(x_{i}^{s s}\right)$ is driven by the difference between the rotation $r$ and total explicit adaptation $\left(x_{e}^{s s}\right)$.

Now consider a system where SPEs drive implicit learning. SPEs (Eq. (2)) are unaltered by strategy. In this case, total implicit learning is determined by Eqs. (2) and (3):

$$
x_{i}^{s s}=\frac{b_{i}}{1-a_{i}+b_{i}} r
$$

Eq. (5) demonstrates an independence between implicit and explicit systems; the total amount of implicit adaptation depends solely on the rotation's magnitude, not one's explicit strategy.

Here we explore how implicit learning systems respond to explicit strategy, and whether behavior is more consistent with competition or independence. Competition and independence can be studied at any point during the adaptation timecourse (Appendix 1). We will primarily examine steadystate learning, where the competition equation (Eq. (4)) and independence equation (Eq. (5)) make simple predictions. The critical insight is that in an independent system (SPE learning), increasing the explicit strategy (Fig. 1B, magenta solid \&dashed) does not alter implicit adaptation (Fig. 1B, independence, compare black \& cyan). However, in a competitive system (Eq. (4)), the same increase in strategy will indirectly decrease implicit learning (Fig. 1B, competition, compare black \& cyan).

To analyze these possibilities, we begin by examining how changes in explicit strategy alter implicit learning in response to variations in rotation magnitude, experimental instructions, rotation type, and at the individual participant level (Part 1). Next, we describe how competition between implicit and explicit systems could in principle mask changes in implicit learning (Part 2). Finally, we will examine studies which suggest implicit error sources vary across experimental conditions due to the presence and/or absence of multiple visual stimuli in the experimental workspace (Part 3).

\section{Part 1: Measuring how implicit learning responds to changes in explicit strategy}

Here, we measure how implicit learning and explicit learning vary across several factors: (1) rotation size, (2) instructions, (3) gradual versus abrupt rotations, and (4) individual subjects. We will ask whether 
the variations in implicit and explicit learning are consistent with the competition or independence theories.

\section{1: Implicit responses to rotation size suggest a competition with explicit strategy}

Over extended exposure to a rotation, adaptation appears to saturate $\mathrm{e}^{1,26,39,40}$. How does implicit learning contribute to steady-state saturation, and what learning model best describes its behavior?

In Neville \& Cressman $(2018)^{30}$, participants adapted to a $20^{\circ}, 40^{\circ}$, or $60^{\circ}$ rotation (Fig. 1C). As is common, adaptation reached a steady-state prior to eliminating the target error $^{26}$ (Fig. 1C, solid vs. dashed lines). To measure implicit learning, participants were instructed to reach to the target without aiming (Fig. 1C, no aiming). The independence model (Eq. (5)) predicts that the implicit response should scale as the rotation increases. On the contrary, total implicit learning was insensitive to rotation size; it reached only $10^{\circ}$ and remained constant despite a three-fold increase in rotation magnitude (Fig. 1D). To estimate explicit strategy, we subtracted the implicit learning measure from the total adapted response. Contrary to implicit learning, explicit strategy increased proportionally with the rotation's size ${ }^{34,35}$ (Fig. $1 E)$.

In the competition model, implicit learning is driven by the difference between the rotation and explicit strategy $\left(r-x_{e}^{s s}\right.$ in Eq. (4)). As a result, when an increase in rotation magnitude is matched by an equal increase in explicit strategy (Fig. 1-Supplement $1 \mathrm{~A}$, same), the implicit learning system's driving force will remain constant (Fig. 1-Supplement $1 B$, same). This constant driving input leads to a phenotype where implicit learning appears to "saturate" with increases in rotation size (Fig. 1Supplement $1 \mathrm{C}$, same).

To investigate whether this mechanism is consistent with the implicit response, we examined how explicit strategy and the implicit driving force varied with rotation size. As rotation size increased, explicit strategies increased substantially (Fig. 1E). Under the competition model, these rapid changes in explicit strategy produced an implicit driving force that responded little to rotation magnitude; while the rotation increased by $40^{\circ}$, the driving force changed by less than $2.5^{\circ}$ (Fig. 1F). Thus, the competition equation (Fig. 1G, competition) suggested that implicit learning would not vary with rotation size, as we observed in the measured data (Fig. 1G, data).

In other words, the competition model suggests that the implicit system can exhibit an unintuitive saturation when its driving input remains constant. The key prediction is that by altering explicit strategy, this driving input will change, altering the implicit response to rotation size. One possibility is to weaken the explicit system's response to the rotation (Fig. 1-Supplement $1 \mathrm{~A}$, slower) which should increase the steady-state of the implicit system (Fig. 1-Supplement 1C, slower).

To test this idea, we used a stepwise rotation ${ }^{25}$. In Experiment 1, participants $(n=37)$ adapted to a stepwise perturbation which started at $15^{\circ}$ but increased to $60^{\circ}$ in $15^{\circ}$ increments (Fig. $1 \mathrm{H}$ ). Twice towards the end of each rotation block, we assessed implicit adaptation by instructing participants to aim directly to the target (Fig. $1 \mathrm{H}$, gray regions). Supplemental analysis suggested that the implicit system reached its steady-state during each learning period (Appendix 2), though this is not required to test the competition theory (Appendix 1.2). Critically, the stepwise rotation onset decreased explicit responses relative to the abrupt rotations used by Neville and Cressman ${ }^{30}$; explicit strategies increased with a $94.9 \%$ gain (change in strategy divided by change in rotation) across the abrupt groups in Fig. $1 \mathrm{E}$, but only a $55.5 \%$ gain in the stepwise condition shown in Fig. $1 \mathrm{~J}$. In the competition model, this 
reduction in strategy increased the implicit system's driving input (Fig. 1K). The increased driving input produced a "scaling" phenotype in the competition model's implicit response (Fig. 1L, competition) which closely matched the measured implicit data (Figs. 1l; 1 L, data; rm-ANOVA, $F(3,108)=99.9, p<0.001$, $\left.\eta_{p}^{2}=0.735\right)$.

Thus, the implicit system can exhibit both saturation (Fig. 1G) and scaling (Fig. 1L), consistent with the competition model. Recent work by Tsay et al. ${ }^{35}$ suggests a third steady-state implicit phenotype: non-monotonicity. In their study, the authors examined a wider range in rotation size, $15^{\circ}$ to $90^{\circ}$ (Fig. 1M). A no-aiming period revealed total implicit adaptation each group ( $n=25 /$ group). Curiously, whereas implicit learning increased between the $15^{\circ}$ and $30^{\circ}$ rotations, it appeared similar in the $60^{\circ}$ rotation group, and then decreased in the $90^{\circ}$ rotation group (Fig. $1 \mathrm{~N}$ ). This non-monotonic behavior was inconsistent with the independence model where implicit learning is proportional to rotation size (Fig. 1Q, independence).

To determine whether this non-monotonicity could be captured by the competition theory, we considered again how explicit re-aiming increased with rotation size (Fig. 10). We observed an intriguing pattern. When the rotation increased from $15^{\circ}$ to $30^{\circ}$, explicit strategy responded with a very low gain (4.5\%, change in strategy divided by change in rotation). An increase in rotation size to $60^{\circ}$ was associated with a medium-sized gain (80.1\%). The last increase to $90^{\circ}$ caused a marked change in the explicit system: a $53.3^{\circ}$ increase in explicit strategy ( $177.7 \%$ gain). Thus, explicit strategy increased more than the rotation had. Critically, this condition produces a decrease in the implicit driving input in the competition theory (Fig. 1-Supplement 1, faster). Overall, we estimated that this large variation in explicit learning gain ( $4.5 \%$ to $80.1 \%$ to $177.7 \%$ ) should yield non-monotonic behavior in the implicit driving input (Fig. 1P): an increase between $15^{\circ}$ and $30^{\circ}$, no change between $30^{\circ}$ and $60^{\circ}$, and a decrease between $60^{\circ}$ and $90^{\circ}$. As a result, the competition theory (Fig. 1Q, competition) exhibited a nonmonotonic envelope, which closely tracked the measured data (Fig. 1Q, data).

Unfortunately, there is a potential problem in our analysis: implicit and explicit learning measures were not independent, because explicit strategy was estimated using implicit reach angles (i.e., explicit learning equals total learning minus implicit learning). Did this bias our analysis towards the competition model? To answer this question, Eq. (4) can be stated as $x_{i}^{s s}=p_{i}\left(r-x_{e}^{s s}\right)$ where $p_{i}$ is the learning gain determined by the implicit system's retention and error sensitivity (i.e., $a_{i}$ and $b_{i}$ ). We can replace the explicit strategy $\left(x_{e}^{s s}\right)$ appearing in this equation noting that $x_{e}^{s s}=x_{T}^{s s}-x_{i}^{s s}$, where $x_{T}^{s s}$ equals total steady-state adaptation. With this, the model relates implicit learning to total learning: $x_{i}^{s s}=p_{i}(1-$ $\left.p_{i}\right)^{-1}\left(r-x_{T}^{s s}\right)$, as opposed to explicit learning, and can be used to test the competition model without correlated learning measures (see Appendix 3). We reexamined all 3 experiments in Fig. 1, using total adaptation to predict implicit learning with the competition model (Fig. 1-Supplement 2). This alternate method yielded nearly identical predictions (Fig. 1-Supplement 2, 'model-2') as Eq. (4) (Fig. 1Supplement 2, 'model-1'). Thus, the qualitative and quantitative correspondence between the competition model and the measured data was not due to how we operationalized implicit and explicit learning (see Appendix 3).

Collectively, these studies demonstrate that the implicit system can exhibit at least three distinct behavioral phenomena: saturation, scaling, or non-monotonicity. The competition model matched all 3 phenotypes, due to the implicit system's response to explicit strategy. The SPE learning model described by the independence equation, however, could only produce a scaling phenotype (Fig. 1I). Could the SPE 
learning model be altered to produce implicit learning phenotypes other than scaling? One possibility is that a saturation phenotype (Fig. 1D) could be built into the SPE model by adding a restriction, i.e., upper bound, on total implicit adaptation, as observed in studies where participants experience invariant error perturbations ${ }^{1,40}$. With that said, the $10^{\circ}$ implicit responses observed across the 3 rotations in Neville and Cressman ${ }^{30}$, are much lower than the $20-25^{\circ}$ ceiling suggested by recent errorclamp studies ${ }^{40}$, and the $35-45^{\circ}$ implicit responses observed in some standard rotation studies ${ }^{36,41}$. More importantly, a learning model with a rotation-insensitive upper bound on implicit learning would be inconsistent with the scaling (Fig. 1I) and nonmonotonic (Fig. 1N; see Appendix 6.6) phenotypes we observed. We will explore other extensions to this SPE model in control analyses in Section 1.6 below.

\section{2: Increase in explicit strategy suppresses implicit learning}

The competition model predicts that increasing explicit strategy will decrease implicit learning, even when the rotation size is the same. In contrast, the independence theory predicts that implicit learning will be insensitive to differences in explicit strategy (extensions to this model are considered in Section 1.6).

To test these ideas, we considered another condition tested by Neville \& Cressman $(2018)^{30}$ where participants were exposed to the same $20^{\circ}, 40^{\circ}$, or $60^{\circ}$ rotation, but received coaching instructions. The coaching sharply improved adaptation over the non-instructed group (Fig. 2A, compare purple with black). To understand how implicit and explicit learning contributed to these changes, we analyzed the mean implicit and explicit reach angles measured across all three rotation sizes (each individual response is shown in Fig. 2-Supplement 1).

Unsurprisingly, adaptation was enhanced in the participants that received coaching instructions. Explicit re-aiming increased by approximately $10^{\circ}$ (Fig. $2 B, t(61)=2.29, p=0.026, d=0.56$ ). However, while instruction enhanced explicit strategy, it suppressed implicit learning, decreasing total implicit learning by approximately $32 \%$ (Fig. $2 \mathrm{C}$, data, $\mathrm{t}(61)=2.62, \mathrm{p}=0.011$, $\mathrm{d}=0.66$ ). To interpret this implicit response, we fit the competition (Eq. (4)) and independence equations (Eq. (5)) to the behavior across all experimental conditions ( 6 groups: 3 rotation magnitudes, 2 instruction conditions), while holding the implicit learning parameters in the model constant (i.e., holding $a_{i}$ and $b_{i}$ constant across all conditions).

As in Fig. 1, implicit learning in the independence model does not respond to explicit strategy, and is not altered by instruction (Fig. 2C, implicit learning, indep.). On the other hand, the competition model accurately suggested that total implicit learning would decrease by approximately $3^{\circ}$ (data showed $2.98^{\circ}$ decrease, model produced a $2.92^{\circ}$ decrease) in response to increases in explicit strategy (Fig. 2C, implicit learning, competition, $\mathrm{t}(61)=2.05, \mathrm{p}=0.045, \mathrm{~d}=0.52$ ). Altogether, the competition theory parsimoniously captured how the implicit system responded to explicit instruction (Fig. 2C) as well as changes in rotation size (Fig. $1 \mathrm{G}$ ) with the same model parameter set (same $a_{i}$ and $b_{i}$ in the competition equation).

\section{3: Decrease in explicit strategy enhances implicit learning}

Next, we examined how implicit learning responds to decreases in explicit strategy. Yin and Wei ${ }^{25}$ recently demonstrated that explicit strategies can be suppressed using gradual rotations. The competition theory predicts that decreasing explicit strategy will lead to greater implicit adaptation. We tested this prediction in Exp. 1. Participants were exposed to a $60^{\circ}$ rotation, either abruptly $(n=36)$, or in 
a stepwise manner $(n=37)$ where perturbation magnitude increased by $15^{\circ}$ across 4 distinct learning blocks (Fig. 2D). We measured implicit and explicit learning during each block, as in Fig. 1. To compare gradual and abrupt learning, we analyzed reach angles during the $4^{\text {th }}$ learning block, where both groups experienced the $60^{\circ}$ rotation size (Fig. 2E).

As in Yin and $\mathrm{Wei}^{25}$, participants in the stepwise condition exhibited a $10^{\circ}$ reduction in explicit re-aiming (Fig. 2F, two-sample t-test, $\mathrm{t}(71)=4.97, \mathrm{p}<0.001, \mathrm{~d}=1.16$ ). Reductions in strategy led to a decrease in total adaptation in the stepwise group by approximately $4^{\circ}$, relative to the abrupt group (Fig. $2 \mathrm{E}$, right-most gray region (last 20 trials); two-sample t-test, $t(71)=3.33, p=0.001, d=0.78$ ), but an increase in implicit learning by approximately 80\% (Fig. 2G, data, two-sample t-test, $t(71)=6.4, p<0.001$, $d=1.5$ ). Thus, the data presented a curious pattern; greater total adaptation in the abrupt condition was paradoxically associated with reduced implicit adaptation. As expected, these surprising patterns did not match the independence model (Fig. 2G, indep.), in which implicit learning does not respond to changes in explicit strategy.

To test whether implicit learning patterns matched the competition model we fit Eq. (4) to implicit and explicit reach angles measured in Blocks 1-4, across the stepwise and abrupt conditions, while holding the model's implicit learning parameters $\left(a_{i}\right.$ and $\left.b_{i}\right)$ constant. The competition model correctly predicted that the decrease in strategy in the gradual condition should produce an increase in implicit learning (Fig. 2G, comp., two-sample t-test, $t(71)=4.97, p<0.001, d=1.16$ ). In addition, the competition model predicted a decrease in total learning, consistent again with the data (the model yielded $53.47^{\circ}$ total adaptation in abrupt, and $50.42^{\circ}$ in gradual: values not provided in Fig. 2). The model's negative correlation between implicit learning and total adaptation occurred in two steps: (1) greater abrupt strategies increased overall adaptation, but (2) siphoned away target errors, reducing implicit adaptation.

We analyzed another hypothesis: changes in implicit adaptation were caused by variation in error sensitivity (e.g., greater implicit error sensitivity in the stepwise condition), rather than competition. Note, however, that the implicit learning gain, $p_{i}$, is given by $p_{i}=b_{i}\left(1-a_{i}+b_{i}\right)^{-1}$. Because the $b_{i}$ term appears in both numerator and denominator, total implicit learning varies slowly with changes in $b_{i}$ (Appendix 4). Accordingly, supplemental analyses (Appendix 4, Fig. 2-Supplement 2) showed that no change in $b_{i}$ could yield the $80 \%$ increase in stepwise implicit learning in Fig. $2 \mathrm{G}$, let alone the $46 \%$ increase in implicit learning in the no-instruction group in Fig. $2 \mathrm{C}$. Thus, while variation in implicit error sensitivity might contribute to changes in steady-learning learning, its role is minor compared to error competition.

In summary, we observed that explicit strategies could be suppressed by increasing the rotation gradually. Reductions in explicit strategy were associated with increased implicit adaptation (Fig. 2G) as predicted by the competition theory. Furthermore, the same competition theory parameter set (i.e., same $a_{i}$ and $b_{i}$, see Methods) accurately matched the extent to which implicit learning responded to decreases in explicit strategy (Fig. 2G) as well as increases in rotation size (Fig. 1L). It is interesting to note that these implicit patterns are broadly consistent with the observation that gradual rotations improve procedural learning ${ }^{33,42}$, although these earlier studies did not properly tease apart implicit and explicit adaptation (see the Saijo \& Gomi analysis described in Appendix 5).

\section{4: Implicit adaptation responds to between-subject differences in explicit adaptation}


Use of explicit strategy is highly variable between individuals ${ }^{12,32,43}$. According to the competition theory (Eq. (4)), implicit and explicit learning will negatively co-vary according to a line whose slope and bias are determined by the properties of the implicit learning system $\left(a_{i}\right.$ and $\left.b_{i}\right)$. In Experiment 2, we tested this prediction. In one group, we limited preparation time to inhibit time-consuming explicit strategies ${ }^{32,44}$ (Figs. 3D-F, PT Limit). In the other group, we imposed no preparation time constraints (Figs. 3A-C, No PT Limit). We measured $a_{i}$ and $b_{i}$ in the Limit PT group and used these values to predict the implicit-explicit relationship across No PT Limit participants.

As expected, PT Limit participants dramatically reduced their reach latencies throughout the adaptation period (Fig. 3F), whereas the No PT Limit participants exhibited a sharp increase in movement preparation time after perturbation onset (Fig. 3C), indicating explicit re-aiming ${ }^{5,13,26,32,44}$. Consistent with explicit strategy suppression, learning proceeded more slowly and was less complete under the PT-Limit (compare Figs. 3B\&E; two-sample t-test on last 10 adaptation epochs: $t(20)=3.27$, $p=0.004, d=1.42$ ).

Next, we measured the retention factor $a_{i}$ during a terminal no feedback period (Fig. 3E, dark gray, no feedback) and error sensitivity $b_{i}$ during the steady-state adaptation period. Steady-state implicit error sensitivity (note errors are small at steady-state creating high $b_{i}$ ) was consistent with recent literature (Fig. 3-Supplements $1 \mathrm{~A}-\mathrm{C})$. Together, this retention factor $\left(a_{i}=0.943\right)$ and error sensitivity $\left(b_{i}=0.35\right)$, produced a specific form of Eq. (4), $x_{i}=0.86\left(30-x_{e}\right)$. We used this result to predict how implicit and explicit learning should vary across participants in the No PT Limit group (Fig. 3G, blue line).

To measure implicit and explicit learning in the No PT Limit group, we instructed participants to move their hand through the target without any re-aiming at the end of the rotation period (Fig. 3B, no aiming). The precipitous change in reaching angle revealed implicit and explicit components of adaptation (post-instruction reveals implicit; voluntary decrease in reach angle reveals explicit). We observed a striking correspondence between the No PT Limit implicit-explicit relationship (Fig. 3G, black dot for each participant; $\rho=-0.95$ ) and that predicted by the competition equation (Fig. 3G, blue). The slope and bias predicted by Eq. (4) (-0.86 and $25.74^{\circ}$, respectively) differed from the measured linear regression by less than $5 \%$ (Fig. 3G, black line, $\mathrm{R}^{2}=0.91$; slope is -0.9 with $95 \% \mathrm{Cl}[-1.16,-0.65]$ and intercept is $25.46^{\circ}$ with $\left.95 \% \mathrm{Cl}\left[22.54^{\circ}, 28.38^{\circ}\right]\right)$.

In addition, we also asked participants to verbally report their aiming angles prior to concluding the experiment. These responses were variable, with $25 \%$ reported in the incorrect direction. Because strategies are susceptible to sign-flipped errors ${ }^{44}$, we assumed these misreported strategies represented the correct magnitude, but the incorrect sign, and thus took their absolute value. While reported explicit strategies were on average greater than our probe-based measure, and report-based implicit learning was on average smaller than our probe-based measure (Figs. 3-Supplement 2A\&B; paired t-test, $t(8)=2.59, p=0.032, d=0.7)$, the two report-based measures exhibited a strong correlation which aligned with the competition theory's prediction (Fig. 3-Supplement $2 \mathrm{C}$; $\mathrm{R}^{2}=0.95$; slope is -0.93 with $95 \% \mathrm{Cl}[-$ $1.11,-0.75]$ and intercept is $25.51^{\circ}$ with $\left.95 \% \mathrm{Cl}\left[22.69^{\circ}, 28.34^{\circ}\right]\right)$.

In summary, individual participants exhibited an inverse relationship between implicit and explicit learning; participants who used large explicit strategies inadvertently suppressed their implicit learning due to error-based competition, a pattern consistent with error-based competition. 


\section{5: Limiting reaction time strongly suppresses explicit strategy and increases implicit learning}

Our analysis in Experiment 2 had two important limitations. First, the competition theory used implicit learning parameters measured under limited preparation time conditions ${ }^{3,32,45}$ : how effectively does this condition suppress explicit learning? Second, our individual-level implicit and explicit learning measures were intrinsically correlated because they both depended on probe-based reach angles (i.e., implicit is no aiming probe, and explicit is total learning minus no aiming probe).

To address these limitations, we conducted a laptop-based control experiment (Experiment 3 ). Participants $(n=35)$ adapted to a $30^{\circ}$ rotation (Fig. 31 ), but this time, we measured implicit adaptation using the no-aiming instruction over an extended 20-cycle period (Fig. 31, no aiming). We calculated early (the first no-aiming cycle; Fig. 3Q) and late (last 15 no-aiming cycles; Fig. 3P) implicit learning measures. Explicit strategy was estimated by subtracting the first no-aiming cycle from total adaptation. Thus, our explicit strategy measure was not calculated using late implicit learning trials; these two measures were no longer spuriously correlated. Regardless, we still observed a strong relationship between explicit strategy and late implicit learning; greater strategy use was associated with reduced late implicit adaptation (Fig. 3P, $\rho=-0.78, p<0.001$ ).

Next, we repeated this experiment, but under limited preparation time conditions in a separate participant cohort (Fig. 3L, Experiment 3, Limit PT, n=21). As for the Limit PT group in Exp. 2, we imposed a strict bound on reaction time to suppress movement preparation time (compare Figs. 3J\&M). Once the rotation period ended, participants were told to stop re-aiming. The decrease in reach angle revealed each participant's explicit strategy (Fig. 3N). When no reaction time limit was imposed (No PT Limit), reaiming totaled $11.86^{\circ}$ (Fig. 3N, E3, black). In addition, we did not detect a statistically significant

difference in re-aiming across Exps. 2 and $3(t(42)=0.50, p=0.621)$. As in earlier reports ${ }^{3,26,32,45}$, limiting reaction time dramatically suppressed explicit strategy, yielding only $2.09^{\circ}$ of re-aiming (Fig. $3 \mathrm{~N}, \mathrm{E} 3$, red). Thus, these data showed that our limited reaction time technique was highly effective at suppressing explicit strategy.

Consistent with the competition theory, suppressing explicit strategy increased implicit learning by approximately $40 \%$ (Fig. 30, No PT-Limit vs. Limit PT, two-sample t-test, t(54)=3.56, $p<0.001, d=0.98$ ). We again used the Limit PT group's behavior to estimate implicit learning parameters $\left(a_{i}\right.$ and $\left.b_{i}\right)$ as we did in Exp. 2 (Fig. 3G). Using these parameters, the competition theory (Eq. (4)) predicted that implicit and explicit adaptation should be related by the line: $x_{i}=0.658\left(30-x_{e}\right)$. As in Exp. 2, we observed a striking correspondence between this model (Fig. 3Q, bottom, model) and the actual implicit-explicit relationship measured in participants in the No PT Limit group (Fig. 3Q, bottom, points). The slope and bias predicted by Eq. (4) (-0.665 and $19.95^{\circ}$, respectively) differed from the measured linear regression by less than $5 \%$ (Fig. $3 Q$, bottom brown line, $\mathrm{R}^{2}=0.78$; slope is -0.63 with $95 \% \mathrm{Cl}[-0.74,-0.51$ ] and intercept is $19.7^{\circ}$ with $\left.95 \% \mathrm{Cl}\left[18.2^{\circ}, 21.3^{\circ}\right]\right)$.

In summary, Exp. 3 provided additional evidence that implicit and explicit systems compete with one another at the individual-participant level. Participants who relied more on strategy exhibited reductions in implicit learning, as predicted by the competition theory. Moreover, by limiting preparation time on each trial, explicit strategies were strongly suppressed, allowing us to estimate the time course of the implicit system's adaptation.

\section{6: Control analyses}


Implicit learning exhibits generalization: a decay in adaptation measured when subjects move to positions across the workspace ${ }^{46-48}$. Implicit generalization is centered where participants aim ${ }^{49,50}$. For this reason, implicit learning measured when aiming towards the target, can underapproximate total implicit learning. Subjects that aim more (larger strategy) can exhibit a larger reduction in measured implicit learning. Might this contribute to the negative implicit-explicit correlations in Exps. 1-3?

To test this idea, we compared our data to generalization curves measured in past studies $47,49,50$ (Fig. 4). Absolute implicit responses are shown in Fig. 4B, and normalized measures are shown in Fig. 4A (see Appendix 6.1). Implicit learning in Exps. 2\&3 declined 300\% more rapidly than predicted by past generalization studies (Fig. 4A\&B). Moreover, this comparison in Figs. 4A-C is not appropriate under the generalization hypothesis. In Exps. 2\&3, explicit strategies are estimated as total learning minus implicit learning. If implicit learning measured at the target underapproximates total implicit learning measured at the aim location, then the explicit strategies we calculate will overapproximate the actual strategy used by each participant. We need to correct these strategies prior to comparing to past generalization curves (Appendix 6.2). The corrected generalization curves (Fig. 4C, E2 and E3 lines) that produce the patterns in Figs. $4 A \& B$ exhibited an unphysiological narrowing: their standard deviation (width) was $85 \%$ smaller than that reported in recent studies ${ }^{47,49,50}$ ( $\sigma$ is about $5.5^{\circ}$ versus $37.76^{\circ}$ in McDougle et al., see Appendix 6.1). These same issues occurred in the group-level phenomena that we analyzed in Figs. 1\&2: no plausible generalization curve could explain the implicit response to instruction, rotation onset (abrupt/gradual), and rotation size (Appendices $6.4 \& 6.5$ ). As an example, the variations in implicit learning across abrupt and stepwise groups in Exp. 1 would require a generalization curve that is $90 \%$ narrower than recent estimates ${ }^{50}$ (see Appendix 6.4 and Fig. 4-Supplement $1 ; \sigma=3.87^{\circ}$ versus $37.76^{\circ}$ in McDougle et al. ${ }^{50}$ ).

We extended the independence model with implicit generalization and compared its behavior to the competition theory. The competition model is given by $x_{i}^{s s}=p_{i}\left(r-x_{e}^{s s}\right)$, where $p_{i}$ is an implicit learning gain. The SPE generalization model is $x_{i}^{\text {measured }}=p_{i} r g\left(x_{e}^{s s}\right)$, where $\mathrm{g}\left(x_{e}^{s s}\right)$ encodes generalization 418 (derivation in Appendix 6.2). We specified $g\left(x_{e}^{s s}\right)$ with McDougle et al. ${ }^{50}$ We considered models where $\mathrm{g}\left(x_{e}^{s S}\right)$ was linear (Figs. 4D-F, SPE gen. linear) and $\mathrm{g}\left(x_{e}^{\text {ss }}\right)$ was normal (SPE gen. normal). Then we fit each model's $p_{i}$ to match implicit learning during the $60^{\circ}$ stepwise rotation in Exp. 1 . We used this gain to predict the implicit-explicit relationship across the three earlier learning periods (B1-B3 in Fig. 4D). The generalization models yielded poor matches to the held-out data (model RMSE in Fig. 4E, rm-ANOVA, $\left.F(2,72)=13.7, p<0.001, \eta_{p}^{2}=0.276\right)$. Further, a model comparison showed that competition best described individual subject data, minimizing AIC in $84 \%$ of stepwise participants (Fig. 4G, Appendix 6.3). Poor SPE generalization model performance was not due to misestimating generalization curve properties; we conducted a sensitivity analysis in which we varied the generalization curve's width. The competition model was superior across the entire range (Fig. 4H, Appendix 6.3).

To understand why the competition theory alone generalized across rotation sizes, we fit linear regressions to the data in each rotation period. The regression slopes and $95 \% \mathrm{Cls}$ are shown in Fig. $4 \mathrm{~F}$ (data). Remarkably, the measured implicit-explicit slope appeared to be constant across all rotation sizes. This invariance was directly consistent with the competition theory (Fig. 4F, competition) which possesses an implicit gain $p_{i}$ that remains constant across rotations (like the data). But in generalization models (Fig. 4F, generalization), the gain relating implicit and explicit learning is not constant; it changes 
as the rotation gets larger (see Appendix 6.3). In sum, data in Exps. 1-3 were poorly explained by an SPE model extended with generalization.

We considered one last control analysis. The competition equation predicts that implicit-explicit correlations are caused by the implicit system's response to variations in strategy. An SPE learning model could create correlations the opposite way: individuals who possess less implicit learning compensate by increasing their explicit strategy. This scenario can be described by $x_{e}^{s s}=p_{e}\left(r-x_{i}^{s s}\right)$ where $p_{e}$ is the explicit response gain. This model has 3 properties (Appendix 7.2). First, implicit and explicit learning will show a negative relationship (Fig. 5A). Second, increases in implicit learning will tend to increase total adaptation (Fig. 5C). Finally, increasing implicit learning leaves smaller errors to drive explicit strategy, resulting in a negative correlation between strategy and total adaptation (Fig. 5B). While the competition model, also predicts negative implicit-explicit correlations (Fig. 5D), the other pairwise correlations differ (Appendix 7.1). Increases in explicit strategy lead to greater total learning (Fig. 5E), but reduce the error which drives implicit learning, leading to a negative correlation between implicit learning and total adaptation (Fig. 5F).

We analyzed these predictions in the No PT Limit group in Exp. 3 (Appendix 7.4). Our observations matched the competition theory; greater explicit strategy was associated with greater total adaptation (Fig. 5G, $\rho=0.84, p<0.001$ ), whereas greater implicit learning was associated with lower total adaptation (Fig. $5 \mathrm{H}, \rho=-0.70, \mathrm{p}<0.001$ ). We repeated these analyses in other datasets (Appendix 7.4) that measured implicit learning with no-aiming probe trials: (1) $60^{\circ}$ rotation groups (combined across gradual and abrupt groups) in Experiment 1, (2) $60^{\circ}$ groups reported by Maresch and colleagues ${ }^{41}$ (combined across the CR, IR-E, and IR-El groups), and (3) $60^{\circ}$ rotation group in Tsay et al. ${ }^{35}$ These data matched the competition theory: negative implicit-explicit correlations (Fig. 5-Supplements 1G-I), positive explicittotal correlations (Fig. 5-Supplements 1D-F), and negative implicit-total correlations (Fig. 5-Supplements $1 \mathrm{~A}-\mathrm{C})$.

In summary, while an SPE learning model could exhibit negative correlations between implicit and explicit adaptation, it does not predict a negative correlation between steady-state implicit learning and total adaptation (nor a positive relationship between steady-state explicit strategy and total adaptation), as we observed in the data. The data were consistent with the competition theory, where the implicit system responds to variations in explicit strategy. However, there is a critical caveat. The predictions outlined above assumed that implicit learning properties, i.e., $p_{i}$, is the same across every participant. This is unlikely to be true, and variation in $p_{i}$ across subjects (e.g., changes in error sensitivity) will undermine some correlations in Fig. 5, particularly the relationship between implicit learning and total adaptation. This phenomenon and past studies where it appears to occur are treated in Appendix 8.

\section{Part 2: Competition with explicit learning can mask changes in the implicit learning system}

Here we show that in the competition model, implicit learning may undergo savings, without changing its learning timecourse. Next, we limit preparation time to detect increases and decreases in implicit learning.

\section{1: Two ways to interpret the implicit response in a savings paradigm}


When participants are exposed to the same perturbation twice, they adapt more quickly the second time. This phenomenon is known as savings and is a hallmark of sensorimotor adaptation ${ }^{27,51,52}$. Multiple studies have attributed this process solely to changes in explicit strategy ${ }^{13,21,22,24,53}$.

For example, in an earlier work ${ }^{13}$, we trained participants $(n=14)$ to reach to one of two targets, coincident with an audio tone (Fig. 6A). By shifting the displayed target approximately $300 \mathrm{~ms}$ prior to tone onset on a minority of trials (20\%), we forced participants to execute movements with limited preparation time (Low preparation time; Fig. 6A, middle). On all other trials (80\%) the target did not switch resulting in high preparation time movements (Fig. 6A, left). We measured adaptation to a $30^{\circ}$ rotation during high preparation time (Fig. 6B, left) and low preparation time trials (Fig. 6B, middle) across two separate exposures (Day 1 and Day 2).

To detect savings, we calculated the learning rate on low and high preparation time trials. Savings appeared to require high preparation time; learning rate increased during the second exposure on high preparation time trials, but not low preparation time trials (Fig. 6B, right; two-way rm-ANOVA, preparation time by exposure number interaction, $F(1,13)=5.29, p=0.039$; significant interaction followed by one-way rm-ANOVA across Days 1\&2: high prep. time with $F(1,13)=6.53, p=0.024$, $\eta_{p}{ }^{2}=0.335$; low preparation time with $\left.F(1,13)=1.11, p=0.312, \eta_{p}{ }^{2}=0.079\right)$. To corroborate this rate analysis, we also measured savings via early changes in reach angle (first 40 trials rotation trials) across Days 1 \& 2 (Fig. 6C, left and middle). Only high preparation time trials exhibited a statistically significant increase in reach angle, consistent with savings (Fig. 6C, right; two-way rm-ANOVA, prep. time by exposure interaction, $F(1,13)=13.79, p=0.003$; significant interaction followed by one-way rm-ANOVA across days: high prep. time with $F(1,13)=11.84, p=0.004, \eta_{p}{ }^{2}=0.477$; low prep. time with $F(1,13)=0.029$, $\mathrm{p}=0.867, \eta_{\mathrm{p}}{ }^{2}=0.002$ ).

Because explicit strategies can be suppressed by limiting movement preparation time under some conditions ${ }^{21,32,44}$, in our initial study we interpreted these data to mean that savings relied solely on time-consuming explicit strategies. Multiple studies have reached similar conclusions ${ }^{13,21,22,24,53}$, suggesting that the implicit learning system is not improved by multiple exposures to a rotation.

However, the competition theory provides an alternate possibility: changes in the implicit learning system may occur but are hidden because of competition with explicit learning. To show this unintuitive phenomenon, we fit the competition model to individual participant behavior under the assumption that low preparation time trials relied solely on implicit adaptation, but high preparation time trials relied on both implicit and explicit adaptation. The model generated implicit (Fig. 6D, blue) and explicit (Fig. 6D, magenta) states that tracked the behavior well on high preparation time trials (Fig. 6D, solid black line) and also low preparation time trials (Fig. 6D, dashed black line).

Next, we considered the implicit and explicit error sensitivities estimated by the model, which are commonly linked to changes in learning rate ${ }^{14,17,19,26,51}$. The model unmasked a surprising possibility: even though savings was observed only on high preparation time trials, but not low preparation time trials (Figs. 6B\&C), the model suggested that both the implicit and explicit systems exhibited a statistically significant increase in error sensitivity (Fig. 6D, right; two-way rm-ANOVA, within-subject effect of exposure number, $F(1,13)=10.14, p=0.007, \eta_{p}{ }^{2}=0.438$; within-subject effect of learning process, $F(1,13)=0.051, p=0.824, \eta_{p}{ }^{2}=0.004$; exposure by learning process interaction, $F(1,13)=1.24, p=0.285$ ).

In contrast, a model where the implicit system adapted to SPEs as opposed to target errors (the independence model) suggested that only the explicit system exhibited a statistically significant increase 
in error sensitivity (Fig. 6E; two-way rm-ANOVA, learning process (i.e., implicit vs explicit) by exposure interaction, $F(1,13)=7.016, p=0.02$; significant interaction followed by 1-way rm-ANOVA across exposures: explicit system, $F(1,13)=9.518, p=0.009, \eta_{p}{ }^{2}=0.423$; implicit system, $F(1,13)=2.328, p=0.151$, $\left.\eta_{\mathrm{p}}^{2}=0.152\right)$.

In summary, when we reanalyzed our earlier data, the competition and independence theories suggested that our data could be explained by two contrasting hypothetical outcomes. If we assumed that implicit and explicit systems were independent, then only explicit learning contributed to savings, as we concluded in our original report. However, if we assumed that the implicit and explicit systems learned from the same error (competition model), then both implicit and explicit systems contributed to savings. Which interpretation is more parsimonious with measured behavior?

\section{2: Competition with explicit strategy can alter measurement of implicit learning}

The idea that implicit error sensitivity can increase without any change in implicit learning rate (Fig. 6) is not intuitive. What the competition model suggests is that when the explicit system increases its learning rate as in Fig. 6D, it leaves a smaller target error to drive implicit learning. However, despite this decrease in target error, low preparation time learning was similar on Days 1 and 2 (Fig. 6B). Because we assumed that low preparation time learning relied on the implicit system, the competition theory required that the implicit system must have experienced an increase in error sensitivity to counterbalance the reduction in target error magnitude. In other words, though increases in implicit error sensitivity did not increase total implicit learning, they still contributed to savings. That is, had implicit error sensitivity remained the same, low preparation time learning would decrease on Day 2, and less overall savings would occur.

To understand how our ability to detect changes in implicit adaptation can be altered by explicit strategy we constructed a competition map (Fig. 7A). Imagine that we want to compare behavior across two timepoints or conditions. Fig. 7A shows how changes in implicit error sensitivity ( $x$-axis) and explicit error sensitivity (y-axis) both contribute to measured implicit aftereffects (denoted by map colors), based on the competition equation (note that the origin denotes a $0 \%$ change in error sensitivity relative to Day 1 adaptation in Haith et al. ${ }^{13}$ ). The left region of the map (cooler colors) denotes combinations of implicit and explicit changes that decrease implicit adaptation. The right region of the map (hotter colors) denotes combinations that increase implicit adaptation. The middle black region represents combinations that manifest as a perceived invariance in implicit adaptation $(<5 \%$ absolute change in implicit adaptation).

This map defines several distinct areas (Fig. 7B). Region A denotes a "matching" decrease between implicit adaptation and error sensitivity; total implicit learning will decline across two separate learning periods due to a reduction in implicit error sensitivity. Region D is similar. Here, total implicit learning will increase across two separate learning periods due to an increase in implicit error sensitivity.

The other regions show less intuitive cases. In Region B, there is a "mismatching" change in total implicit learning and implicit error sensitivity; here total implicit learning decreases even though implicit error sensitivity has increased or stayed the same. Likewise, in Region E, total implicit learning will increase across two separate learning periods, though implicit error sensitivity has decreased or stayed the same. 
Indeed, we have already described these cases in Fig. 2. For example, by enhancing the explicit system via coaching (Figs $2 A-C$ ), implicit learning decreased. This scenario is equivalent to moving up the $y$-axis of the map (Fig. 7C, top). The same implicit system will decrease its output (Fig. 7C, bottom) when normal levels of explicit strategy are increased (Fig. 7C, middle). On the other hand, suppressing explicit strategy by gradually increasing the rotation (Figs. 2D-G), or limiting reaction time (Figs. 3N\&O), increased implicit learning without changing any implicit learning properties. This scenario is equivalent to moving down the $y$-axis of the competition map (Fig. 7D, top). The same implicit system will increase its output (Fig. 7D, bottom) when normal levels of explicit strategy are then suppressed (Fig. 7D, middle).

Now, let us consider the savings experiment in Fig. 6. The competition theory predicted (Fig. 6D) that explicit error sensitivity increased by approximately $70.6 \%$ during the second exposure, whereas the implicit system's error sensitivity increased by approximately $41.5 \%$ (Fig. 7E, middle). These changes in implicit and explicit adaptation describe a single point in the competition map, denoted by the gray circle in Fig. 7E (top). This experiment occupies Region C, which indicates that despite the $41.5 \%$ increase in implicit error sensitivity, the total implicit learning will increase by less than 5\% (Fig. 7E, bottom). In other words, the competition model suggests the possibility that implicit learning improved between Exposures 1 and 2, but this change was hidden by a dramatic increase in explicit strategy.

To test this prediction, we can suppress explicit adaptation, thus eliminating competition (Fig. $7 F$, middle). Such an intervention would move our experiment from Region C to Region D (Fig. 7F, top) where we will observe greater change in the implicit process (Fig. 7D, bottom). We examined this possibility in a new experiment.

\section{3: Savings in implicit learning is unmasked by suppression of explicit strategy}

In Exp. 4 (Fig. 8), participants experienced two $30^{\circ}$ rotations, separated by washout trials with veridical feedback (mean reach angle over last three washout cycles was $0.55 \pm 0.47^{\circ}$, 1-sample t-test against zero, $t(9)=1.16, p=0.28$; not shown in Fig. 8). To suppress explicit strategy, we restricted reaction time on every trial, which in Exp. 3, greatly reduced explicit learning (Fig. $3 \mathrm{~N}$; re-aiming decreases from $12^{\circ}$ to about $2^{\circ}$ ). Under these reaction time constraints, participants exhibited reach latencies around $200 \mathrm{~ms}$ (Fig. 8B, top).

While limiting preparation time prevented savings in Haith et al. ${ }^{13}$ (Fig. 8A, low preparation time on $20 \%$ of trials), inhibiting strategy use on every trial in Experiment 4 yielded the opposite outcome (Fig. 8B). Low preparation time learning rates increased by more than $80 \%$ in Experiment 4 (Fig. $8 \mathrm{C}$ top; mixed-ANOVA exposure number by experiment type interaction, $F(1,22)=5.993, p=0.023$; significant interaction followed by one-way rm-ANOVA across exposures: Haith et al. with $F(1,13)=1.109, p=0.312$, $\eta_{p}{ }^{2}=0.079$; Experiment 4 with $\left.F(1,9)=5.442, p=0.045, \eta_{p}{ }^{2}=0.377\right)$. Statistically significant increases in reach angle were detected immediately following rotation onset in Experiment 4 (Fig. 8B, bottom), but not our earlier data (Fig. 8C, bottom; mixed-ANOVA exposure number by experiment interaction, $F(1,22)=4.411, p=0.047$; significant interaction followed by one-way rm-ANOVA across exposures: Haith et al. with $F(1,13)=0.029, p=0.867, \eta_{p}{ }^{2}=0.002$; Experiment 4 with $F(1,9)=11.275, p=0.008, \eta_{p}{ }^{2}=0.556$ ).

In sum, when explicit learning was inhibited on every trial, low preparation time behavior showed savings (Fig. 8B). But when explicit learning was inhibited less frequently, low preparation time behavior did not exhibit a statistically significant increase in learning rate (Fig. 8A). The competition 
theory provided a possible explanation; that an implicit system expressible at low preparation time exhibits savings, but these changes in implicit error sensitivity can be masked by competition with explicit strategy.

However, the savings we measured at limited preparation time may not be solely due to changes in implicit learning, but also cached explicit strategies ${ }^{21,44}$. Indeed, when we limited preparation time in Exp. 3, participants still exhibited a small decrease $\left(2.09^{\circ}\right)$ in reach angle when we instructed them to stop aiming (Fig. 3L, no aiming; Fig. 3N, E3, red). These small residual strategies could have contributed to the $8^{\circ}$ reach angle measured early during the second rotation in Exp. 4 (Fig. 8C, implicit difference, no comp.).

What that said, the "aiming angle" we measured in the Limit PT group in Exp. 3, may overestimate the extent to which participants can use explicit strategy in our limited preparation time paradigm. That is, the decrease in reach angle we observed when participants were told to stop aiming (Fig. 3L, no aiming) may be due to time-based decay in implicit learning ${ }^{30,41}$ over the 30 second instruction period, as opposed to a voluntary reduction in strategy.

To test this alternate interpretation, we collected another limited preparation group ( $n=12$, Fig. 8-Supplement $1 \mathrm{~A}$, decay-only, black). But this time, participants were instructed that the experiment's disturbance was still on, and that they should continue to move the 'imagined' cursor through the target during the terminal no feedback period. Despite this instruction, reach angles decreased by approximately $2.1^{\circ}$ (Fig. 8-Supplement 1B, black). Indeed, we detected no statistically significant difference between the change in reach angle in this decay-only group, and the Limit PT group in Experiment 3 (Fig. 8-Supplement 1B; two-sample t-test, t(31)=0.016, $\mathrm{p}=0.987$ ).

This control experiment suggested that 'explicit strategies' we measured in the Limit PT condition were more likely caused by time-dependent decay in implicit learning. Indeed, our Limit PT protocol may eliminate explicit strategy. This additional analysis lends further credence to the hypothesis that savings in Experiment 4 was primarily due to changes in the implicit system rather than cached explicit strategies.

\section{4: Impairments in implicit learning contribute to anterograde interference}

Exp. 4 suggested that the implicit system can exhibit savings. We next wondered whether these changes are bidirectional: can the implicit learning rate decrease? When subjects learn two opposing perturbations in sequence, their adaptation slows due to another hallmark of adaptation, anterograde interference.

In Experiment 5, we exposed two groups of participants to opposing visuomotor rotations of $30^{\circ}$ and $-30^{\circ}$ in sequence (Experiment 5 ). In one group, the perturbations were separated by a 5 -minute break (Fig. 9A). In a second group, the break was 24 hours in duration (Fig. 9B). We inhibited explicit strategies by strictly limiting reaction time. Under these constraints, participants executed movements at latencies near $200 \mathrm{~ms}$ (Figs. 9A\&B, middle, blue). These reaction times were approximately 50\% lower than those observed when no reaction time constraints were imposed on participants, as in our earlier work $^{19}$ (Figs. 9A\&B, middle, green).

To assess changes in low preparation time learning, we measured the adaptation rate during each rotation period. In addition, we re-analyzed the adaptation rates obtained in our earlier work ${ }^{19}$ where participants were tested in a similar paradigm but without any reaction time constraints. While 
both low preparation time and high preparation time trials exhibited decreases in learning rate which improved with the passage of time (Fig. 9C; two-way ANOVA, main effect of time delay, $F(1,50)=5.643$, $p=0.021, \eta_{p}^{2}=0.101$ ), these impairments were greatly exacerbated by limiting preparation time (Fig. 9C; two-way ANOVA, main effect of preparation time, $\left.F(1,50)=11.747, p=0.001, \eta_{p}{ }^{2}=0.19\right)$. This result was unrelated to initial differences in error across rotation exposures; we obtained analogous results (see Methods) when learning rate was calculated after the 'zero-crossing' in reach angle (two-way ANOVA, main effect of time delay, $F(1,50)=4.23, p=0.045, \eta_{p}{ }^{2}=0.067$; main effect of prep. time, $F(1,50)=8.303$, $\mathrm{p}=0.006, \eta_{\mathrm{p}}^{2}=0.132$ ).

Thus, inhibiting explicit strategy via preparation time constraints revealed a strong and sustained anterograde deficit in implicit learning. Under normal reaction time conditions, adaptation rates were less impaired, suggesting that explicit strategies may have partially compensated and masked lingering deficits in the implicit system's sensitivity to error.

\section{Part 3: Limitations of the Competition Theory}

The competition theory assumes that learning in the implicit system is driven by only one error. Here we show that this single error hypothesis is unlikely to be true in every condition. To demonstrate the theory's limitations, we examine two earlier studies and speculate how the theory might be extended to account for these more sophisticated behaviors.

\section{1: The implicit system may adapt to multiple target errors at the same time}

In Mazzoni and $\mathrm{Krakauer}^{2}$, we tested two sets of participants. In a no-strategy group, participants adapted to a standard $45^{\circ}$ rotation (Fig. 10A, blue, no-strategy, adaptation) followed by washout (Fig. $10 \mathrm{~A}$, blue, no-strategy, washout). In a second group, participants made two initial movements with the rotation (Fig. 10A, red, strategy, 2 movements no instruction). Then we coached subjects to aim towards a neighboring target $\left(45^{\circ}\right.$ away) which entirely compensated for the rotation. Participants adopted the aiming strategy, bringing the primary target error to zero (Fig. 10A, red, strategy, instruction). Curiously, even though the primary target error had now been eliminated, reaching movements gradually drifted beyond the primary target, overcompensating for the rotation. These involuntary changes implicated an implicit process.

When we compared the rate of learning with and without strategy in Mazzoni and Krakauer², we found that it was not different during the initial exposure to the perturbation (Fig. 10B, gray, mean adaptation over rotation trials $1-24$, Wilcoxon rank sum, $p=0.223$ ). This statistical test led us to conclude in Mazzoni and Krakauer, that implicit adaptation was driven by a sensory prediction error that did not depend on the primary target and was not altered by explicit strategy.

However, there remained an unsolved puzzle. While the initial rates of adaptation were the same irrespective of strategy, adaptation diverged later in learning (Fig. 10B, compare strategy and nostrategy curves after initial gray region; two-sample t-test, $p<0.005)$, with the no-strategy group exhibiting a larger aftereffect (see aftereffect in Fig. 10C; two-sample t-test, $p<0.005$ ). Might these late differences have been caused by participants in the strategy group abandoning their explicit strategy as it led to larger and larger errors? This possibility seemed unlikely. When we asked participants to stop using their aiming strategy and to move instead toward the primary target (Fig. 10A, do not aim rotation on) their movement angle changed by $47.8^{\circ}$ (difference between 3 movements before and 3 
movements after instruction), indicating that they had continued to maintain the instructed explicit reaiming strategy near $45^{\circ}$.

We wondered if interactions between implicit and explicit learning could help solve this puzzle. First, we considered the competition model that best described the experiments in Figs. 1-7. In this model, the implicit system is driven exclusively by error with respect to the primary target (Eq. (1)) (Fig. 10D, top, $e_{1}$ ). While this model predicted learning in the standard no-strategy condition, it failed to account for the drift observed when participants were given an explicit strategy (Fig. 10D, no learning in strategy group). This was not surprising. If implicit learning is driven by the primary target's error, it will not adapt in the strategy group because participants explicitly reduce target error to zero at the start of adaptation (note that $45^{\circ}$ in Fig. 10D means a $0^{\circ}$ primary target error).

We next considered the possibility that implicit learning was driven exclusively by an error with respect to the aimed target (target 2 , Fig. 10E, top, $e_{2}$ ), as we concluded in our original study ${ }^{2}$. While this model correctly predicted non-zero implicit learning in the no-strategy and strategy groups, it could not account for any differences in learning that emerged later during the adaptation period (Fig. 10E, bottom).

Finally, we noted that participants in the strategy group were given two contrasting goals. One goal was to aim for the neighboring target, whereas the other goal was to move the cursor through the primary target (both targets were always visible). Therefore, we wondered if participants in the strategy group learned from two distinct target errors: cursor with respect to target 1, and cursor with respect to target 2 (Fig. 10F, top). In contrast, participants in the no-strategy group attended solely to the primary target, and thus learned only from the error between the cursor and target 1 . Thus, we imagined that implicit learning in the strategy group was driven by two target errors: $e_{1}$ was cursor with respect to target 1 , and $e_{2}$ was cursor with respect to target 2 :

$$
\begin{aligned}
& x_{i, 1}^{(n+1)}=a_{i} x_{i, 1}^{(n)}+b_{i} e_{1}^{(n)} \\
& x_{i, 2}^{(n+1)}=a_{i} x_{i, 2}^{(n)}+b_{i} e_{2}^{(n)}
\end{aligned}
$$

These two modules then combined to determine the total amount of implicit learning (i.e., $x_{i}=x_{i, 1}+x_{i, 2}$ ).

Interestingly, when we applied the dual target error model (Eq. (6)) to the strategy group, and the single target error model (Eqs. (1) \& (3)) to the no-strategy group, the same implicit learning parameters $\left(a_{i}\right.$ and $\left.b_{i}\right)$ closely tracked the observed group behaviors (black model in Fig. 10B). These models correctly predicted that initial learning would be similar across the strategy and no-strategy conditions but would diverge later during adaptation (Fig. 10F). How was this possible?

In Fig. 10G, we show how the primary target error and aiming target error evolved over time in the instructed strategy group. Initially, strategy reduces primary target error to zero (Fig. 10G, primary target error). Thus, early in learning, the implicit system is driven predominantly by aiming target error. For this reason, initial learning will appear similar to the no-strategy group which also adapts to only one error. However, as the error with respect to the aimed target decreases, error with respect to the primary target increases but in the opposite direction (Fig. 10G; see schematic in Fig. 10F). Therefore, the primary target error opposes adaptation to the aiming target error. This counteracting force causes implicit adaptation to saturate prematurely. Hence, participants in the no-strategy group, who do not experience this error conflict, adapt more. 
It is important, however, to note a limitation in these analyses. Our earlier study did not employ the standard conditions used to measure implicit aftereffects: i.e., instructing participants to aim directly at the target, and also removing any visual feedback. Thus, the proposed model relies on the assumption that differences in washout were primarily related to the implicit system. These assumptions need to be tested more completely in future experiments.

In summary, the conditions tested by Mazzoni and Krakauer show that the simplistic idea that adaptation is driven by only one target error, or only one SPE, cannot be true in general ${ }^{54}$. We propose a new hypothesis that when people move a cursor to one visual target, while aiming at another visual target, cursor error with respect to each target contributes to implicit learning. When one target error conflicts with the other target error, the implicit learning system may exhibit an attenuation in total adaptation.

This experiment alone does not reveal the nature of aiming target error. That is, in the strategy group, the error between the aim direction and the cursor is both an SPE, but also a target error (because participants are aiming at a neighboring target). We explore this distinction in the next section.

\section{2: The persistence of sensory prediction error, in the absence of target error}

Our analysis in Figs. 10A-G suggested that when participants see two targets, one to aim toward with their hand and one to move the cursor to, the landmarks can act as two different target errors. To what extent do these errors depend on the target's physical presence in the workspace? Taylor \& Ivry ${ }^{8}$ tested this idea, repeating the instruction paradigm used by Mazzoni and Krakauer, though with nearly 4 times the number of adaptation trials (Fig. 10l, instruction with target, black). Interestingly, while the reach angle exhibited the same implicit drift described by Mazzoni and Krakauer, with many more trials participants eventually counteracted this drift by modifying their explicit strategies, bringing their target error back to zero (Fig. 10l, black). At the end of adaptation, participants exhibited large implicit aftereffects when instructed to stop aiming (Fig. 10I, right, aftereffect; $t(9)=5.16, p<0.001$, Cohen's $d=1.63$ ).

In a second experiment, participants were taught how to re-aim their reach angles during an initial baseline period, but during adaptation itself, they were not provided with physical aiming targets (Fig. 10I, instruction without target). In this case, only SPEs (not a target error) could drive implicit learning towards the aimed location. Even without physical aiming landmarks, participants immediately eliminated error at the primary target after being instructed to re-aim (Fig. 10l, middle, yellow). Curiously, without the physical aiming target, these participants did not exhibit an implicit drift in reach angle at any point during the adaptation period and exhibited only a small implicit aftereffect during the washout period (Fig. 10I, right, $t(9)=3.11, p=0.012$, Cohen's $d=0.985$ ). In fact, the aftereffect was approximately 3 times larger when participants aimed towards a physical target during adaptation than when this target was absent (Fig. 10l, right, aftereffect; two-sample t-test, $t(18)=2.85, p=0.012$, Cohen's $d=0.935$ ).

A target error (competition) model is consistent with some of these results, but not all. The model correctly predicts that when only a single target is present, performance during adaptation will not exhibit a drift, even though people are aiming. However, it does not explain why this condition still leads to the small aftereffect. Further, with two targets, it correctly predicts that adaptation will drift, as 
in Mazzoni \& Krakauer, but it does not explain how this is eliminated late during adaptation; this reversal in drift would seem to indicate a compensatory and gradual reduction in explicit strategy ${ }^{8,11,37}$.

Together, the data suggested a remarkable depth to the implicit system's response to error. While implicit learning was greatest in response to target error, removing the physical target still permitted SPE-driven learning, albeit to a smaller degree. Whether this aiming-related error is both a target error and an SPE occurring together, or solely an SPE enhanced by a salient visual stimulus, remains unknown.

\section{Discussion}

Sensorimotor adaptation relies on an explicit process shaped by intention ${ }^{37,55}$, and an implicit process driven by unconscious correction ${ }^{1,2,40}$. Here we examined the possibility that these two parallel systems can become entangled when they respond to a common error source: target (i.e., task) error ${ }^{3,10}$. The data suggested that this coupling resembles a competition by which enhancing the explicit system's response rapidly depletes error, decreasing the driving force for implicit adaptation. Thus, providing instructions on how to reduce errors enhances the explicit system, but comes at the cost of robbing the implicit system from what it needs to adapt.

This simple rule explained why the implicit system can operate in three modes, one that appears insensitive to perturbation magnitude, another that scales with the perturbation's size, and a third that exhibits non-monotonic behavior (Fig. 1). It also predicted that priming or suppressing explicit awareness can inversely change implicit adaptation (Fig. 2). As a result, subjects that utilize strategies inadvertently suppress their implicit learning (Figs. 3-5). This inhibition can continue to the extent that improvements in implicit learning (e.g., savings) are masked by dramatic upregulation in strategic learning (Figs. 6-8).

The task-error driven implicit system likely exists in parallel with other implicit processes $3,10,56$. For example, in cases where primary target errors are eliminated, small amounts of implicit adaptation persist (Fig. 10). These residual changes are likely due to sensory prediction errors ${ }^{2,3,8,10}$ as well as other target errors that remain in the workspace (Fig. 10I). When these error sources oppose one another, competition between parallel implicit learning modules may inhibit the overall implicit response (Figs. $10 \mathrm{~A}-\mathrm{C})$.

In a broader sense, these competitive interactions extend beyond implicit and explicit processes, to other parallel neural circuits that respond to a common error. Changes in one neural circuit's response to error may be indirectly driven, or hidden, by a parallel circuit. Thus, competition may lead to long-range interactions between neuroanatomical regions that subserve separate neural processes. For example, strategic learning systems housed within the cortex ${ }^{38,57,58}$, may exert indirect changes on a subcortical structure like the cerebellum, which is widely implicated in subconscious adaptation ${ }^{6,59-64}$.

\section{Flexibility in the implicit response to error and its contribution to savings}

When two similar perturbations are experienced in sequence, the rate of relearning is enhanced during the second exposure ${ }^{13,14,17,52,65}$. This hallmark of memory ${ }^{66,67}$ is referred to as savings, which is often quantified based on differences in the learning curves for each exposure ${ }^{13,22}$, or the rate of adaptation ${ }^{68}$. These conventions are based on an underlying assumption: when a learning system is enhanced, its total adaptation will also change. Here we showed that this intuition is incorrect. 
The state space model ${ }^{27-29}$ quantified behavior using two processes: learning and forgetting. This model described savings as a change in sensitivity to error ${ }^{14,17,51}$. When similar errors are experienced on consecutive trials, the brain becomes more sensitive to their occurrence and responds more strongly on subsequent trials ${ }^{26,51,69}$. Generally, as error sensitivity increases, so too does the rate at which we adapt to the perturbation (e.g., High PT trials in Fig. 6). However, under certain circumstances, changes in one's implicit sensitivity to error may not lead to differences in measured behavior (e.g., Low PT trials in 812 Fig. 6).

The reason is competition. When strategy is enhanced, it reduces the error available for implicit

\section{sensitivity is canceled out by the decrease in error size.}

For example, recent lines of work have suggested that increases in learning rate depend solely on the explicit recall of past actions. Implicit adaptation does not seem to contribute to faster relearning, whether implicit learning is estimated via reported strategies ${ }^{22}$, or by intermittently restricting movement preparation time ${ }^{13,21}$ (Fig. 6). These results suggested that implicit processes do not show savings. Our data suggest a different possibility. When we limited reaction time on all trials in Experiment 4, thus suppressing explicit contributions to behavior, we found that the implicit system exhibited savings (Fig. 8). The disconnect between studies that have detected changes in both implicit and explicit learning rates ${ }^{3,25,26}$, versus studies that have only observed changes in explicit learing ${ }^{13,21-24}$, can be resolved by the competition equation (Eq. (4)).

The competition equation links steady-state implicit learning to both implicit and explicit learning properties (Fig. 7). When both implicit and explicit systems become more sensitive to error, the explicit response can hide changes in the implicit response (Fig. 7B, Region C). Moreover, dramatic enhancement in explicit adaptation could even lead to a decrease in implicit learning, even when implicit error sensitivity has increased (Fig. 7B, Region B). Indeed, this prediction can explain cases whereby re-exposure to a rotation increases explicit strategies, but can attenuate implicit learning ${ }^{21,24,70}$. For example, in a recent study by Huberdeau and colleagues ${ }^{21}$, seven exposures to a rotation dramatically enhanced the strategic learning system, but simultaneously attenuated implicit learning. Prolonged multi-day exposure to a rotation appears to have a similar outcome ${ }^{70}$.

It is critical to distinguish between cases where implicit learning is indirectly reduced by increases in explicit strategy, versus contexts that lead to direct impairments in the implicit system's sensitivity to error. For example, when two opposing perturbations are experienced sequentially, the response to the second exposure is impaired by anterograde interference ${ }^{18,20,27,71}$. Recently, we linked these impairments in learning rate to a transient reduction in error sensitivity which recovers over time ${ }^{19}$. Here, we limited reaction time to try and isolate the implicit contributions to this impairment. Impairments in learning at low preparation time were long-lasting, persisting even 24 hours, and exceeded those measured at normal movement preparation times (Fig. 9C). These results suggested that less-inhibited explicit strategies may sometimes compensate, at least in part, for lingering deficits in implicit adaptation ${ }^{3,21}$. Our analysis in Fig. 9, however, compares Exp. 5 to our earlier work in Lerner et al. ${ }^{19}$ where we did not tease apart implicit and explicit learning. Thus, future work needs to test these ideas more carefully.

There is a possible limitation in this interpretation. Recent studies have demonstrated that with multiple exposures to a rotation, explicit responses can be expressed at lower reaction times: a process 
termed caching ${ }^{21,44}$. Thus, changes in low preparation time adaptation commonly ascribed to the implicit system, may be contaminated by cached explicit strategies. This possibility seems unlikely to have altered our results. First, it is not clear why caching would occur in Experiment 4, but not our earlier study in Haith et al. ${ }^{13}$ (Fig. 8); these earlier data implied that caching remains limited with only two exposures to a rotation (at least during the initial exposure to the second rotation over which savings was assessed). Nevertheless, to test the caching hypothesis, we measured explicit re-aiming under limited preparation time conditions in Experiment 3. We found that our method restricted explicit reaiming to only $2^{\circ}$, compared to about $12^{\circ}$ in the standard condition (Fig. $3 \mathrm{~N}$ ). Moreover, this $2^{\circ}$ decrement in reach angle was more likely due to forgetting in implicit learning ${ }^{30,72-76}$. That is, we measured a similar $2^{\circ}$ decrease in reach angle occurred over the $30 \mathrm{sec}$ instruction period, even when participants were not told to stop aiming (Fig. 8-Supplement 1). Thus, while it appears that caching played little role in our results, our results should be taken cautiously. It is critical that future studies investigate how caching varies across experimental methodologies, and how cached strategies interact with implicit learning. In addition, such experiments should dissociate these cached explicit responses from associative implicit memories that may be rapidly instantiated in the appropriate context.

\section{Competition-driven enhancement and suppression of implicit adaptation}

The competition theory cautions that increases or decreases in implicit learning do not necessarily imply that the implicit system has altered its response to error. That is, changes in implicit learning may occur indirectly through competition with explicit strategies.

For example, when participants are coached about a visuomotor rotation prior to its onset, their explicit strategies are greatly enhanced ${ }^{30,31}$. These increases in explicit strategy are coupled to decreases in implicit adaptation (Fig. 2). A similar phenomenon is observed in other experiments where participants report their strategy using visual landmarks. In such paradigms, increased reporting frequency leads to increased explicit strategy, but decreased implicit learning ${ }^{41,43,77}$. Subjects themselves exhibit substantial variations in strategic learning, leading to negative individual-level correlations between implicit and explicit learning ${ }^{30-32}$ (Fig. 3).

The competition theory helps to reveal the input that drives implicit learning. This competitive relationship (Eq. (4)) naturally arises when implicit systems are driven by errors in task outcome (Eq. (1)). We can observe these negative interactions not solely when enhancing explicit strategy, but also when suppressing re-aiming. For example, in cases where perturbations are introduced gradually, thus reducing conscious awareness, implicit "procedural" adaptation appears to increase ${ }^{25,33,42}$ (Fig. 2, Fig. 2Supplement 3, and Appendix 5). Similarly, when participants are required to move with minimal preparation time, thus suppressing time-consuming explicit re-aiming ${ }^{13,32,44}$, the total extent of implicit adaptation also appears to increase (Fig. 30) ) $^{26,32}$.

Although the implicit system varies with experimental conditions, a common phenomenon is its invariant response to changes in rotation size $e^{1,30,34,35,40}$. For example, in the Neville and Cressman ${ }^{30}$ data examined in Fig. 1, total implicit learning remained constant despite tripling the rotation's magnitude. While this saturation in implicit learning is sometimes due to a restriction in implicit adaptability ${ }^{1,40}$, in some cases this rotation-insensitivity may have another cause entirely: competition. That is, when rotations increase in magnitude, rapid scaling in the explicit response may prevent increases in total implicit adaptation. In the competition theory, implicit learning is driven not by the rotation, but by the 
residual error that remains between the rotation and explicit strategy. Thus, when we used gradual rotations to reduce explicit adaptation (Experiment 1), prior invariance in the implicit response was lifted: as the rotation increased, so too did implicit learning ${ }^{36}$ (Fig. 11). The competition theory readily described these two implicit learning phenotypes: saturation and scaling (Figs. 1G\&L). Furthermore, it also provided insight as to why implicit learning can even exhibit a non-monotonic response, as in Tsay et al. $(2021)^{35}$.

With that said, changes in implicit learning occur not solely due to error-based competition, but also variations in implicit learning properties such as error sensitivity. For example, Morehead et al. ${ }^{1}$ show that total implicit learning paradoxically decreases when rotations exceed about $90^{\circ}$. A possible cause is error sensitivity, which declines as errors become larger ${ }^{40,78,79}$. Because no aiming was permitted in their study, steady-state errors were $>80^{\circ}$, which would dramatically reduce error sensitivity. Reductions in error sensitivity could contribute to the non-monotonic phenotype we described in Tsay et al. (2021). On the other hand, Tsay et al. permitted aiming, so steady-state errors were only about $5^{\circ}$ in the $90^{\circ}$ rotation group. These residual errors would not be associated with dramatic reduction in error sensitivity, so error-based competition seems a more likely mechanism (Fig. 1Q). In addition, note that total implicit learning varies strongly with error but not error sensitivity; the implicit learning gain $p_{i}=$ $b_{i}\left(1-a_{i}+b_{i}\right)$, responds weakly to changes in $b_{i}$ (see Appendix 4). Thus, large changes in total implicit learning are much more likely driven by a competition for error, than by changes in implicit error sensitivity (Appendix 6.6 provides additional comparisons between Morehead et al. and Tsay et al.).

In addition, there may be other ways to cast the adaptation model, that also produce competition between implicit learning and explicit strategy. Here, implicit and explicit systems are treated as parallel states that adapt to the same error. A recent inference-based model of motor adaptation ${ }^{80}$ suggests the possibility that implicit and explicit systems participate in a credit assignment problem: with the explicit state estimating the external perturbation, and implicit state estimating the mismatch between vision and proprioception. This inference model will also produce a competition because both states attempt to sum to total state feedback. When more credit is assigned to the external perturbation, explicit adaptation will increase, and implicit adaptation will decrease. All in all, this model will produce similar phenotypes to the competition equation, given that they both describe a competitive learning process.

Variations in individual learning unveil competition between implicit and explicit processes Individuals exhibit substantial variation in how they adapt to rotations ${ }^{12,32,81}$. For example, in Experiments 1-3, we observed that individuals who relied more on explicit strategy inadvertently suppressed their own implicit learning. In one prime example, Miyamoto and colleagues ${ }^{12}$ exposed participants to sum-of-sines rotations. Curiously, participants with more vigorous explicit responses to the perturbation exhibited less vigorous implicit learning. In a second case, Fernandez-Ruiz and colleagues $^{32}$ observed that increases in movement preparation time helped participants adapt more rapidly, but led to reductions in aftereffects. As a third example, when Bromberg et al. ${ }^{43}$ measured eye movements during adaptation, participants who tended to look towards their re-aiming locations not only exhibited greater explicit strategies, but less implicit adaptation.

These results suggest that a subject's strategy suppresses their implicit learning ${ }^{32}$. To explain these individual correlations, Miyamoto et al. ${ }^{12}$ suggested that there may be an intrinsic relationship 
between implicit and explicit sensitivity to error: when an individual's explicit error sensitivity is high, their implicit error sensitivity is low. Here our results describe another way to account for a similar observation (Fig. 3). In Exps. 2 and 3, we used the competition equation (Eq. (4)) to predict an individual's implicit adaptation from their measured explicit strategy, assuming each participant had the same sensitivity to error. This equation could accurately predict the negative relationship between implicit and explicit learning. Thus, negative individual-level correlations between implicit and explicit adaptation can arise from variation in strategy, even under an extreme scenario where implicit error sensitivity is constant across participants.

There are alternate ways that such negative correlations between implicit and explicit learning might arise. For example, here we described an implicit-centered competition equation where explicit strategies suppress implicit learning. The opposite is also possible; implicit learning might be immune to explicit strategy, but strategies respond to variation in implicit learning. These contrasting possibilities both predict negative relationships between implicit learning and explicit strategy but diverge in how total adaptation should vary with implicit and explicit states (Fig. 5). When we tested these ideas in Experiment 3, our data were highly consistent with the competition model: increases in total learning were associated with greater strategy, but less implicit learning (Figs. 5G\&H). We observed similar phenomena across 3 additional studies (Fig. 5-Supplement 1). Thus, in cases where implicit learning is dominated by target errors, greater total adaptation may be supported by less implicit learning. Note, however, that negative correlations at the individual-level are more nuanced. Variation in implicit learning properties will weaken the relationship between implicit learning and total adaptation (Appendices 7 \& 8). Further, in conditions with enhanced SPE learning (e.g., multiple visual landmarks), these correlations can easily be invalidated.

These results imply that implicit learning responds to variations in explicit strategy, but strategies are immune to implicit learning. A similar phenomenon was noted by Miyamoto et al. ${ }^{12}$, using structural equation modeling. This unidirectional causality, however, is not true in general. For example, early during learning, it is common that explicit strategies increase, peak, and then decline. That is, when errors are initially large, strategies increase rapidly. But as implicit learning builds, the explicit system's response can decline in a compensatory manner ${ }^{8,11,37}$. This dynamic phenomenon can also occur in the competition theory, where both implicit and explicit systems respond to target error (Fig. 6D). But in many cases, a second error source may drive this behavioral phenotype. That is, in cases with aiming landmarks ${ }^{8,11,37}$, errors between the cursor and primary target can be eliminated, but implicit learning persists. This implicit learning is likely driven by SPEs and target errors that remain between the cursor and aiming landmark ${ }^{8}$. Persistent implicit learning is counteracted by decreasing explicit strategy to avoid overcompensation. In sum, competition between implicit learning and explicit strategy is complex. Both systems can respond to one another in ways that change with experimental conditions.

\section{Comparisons to invariant error-clamp experiments}

The competition and independence models described here apply solely to standard visuomotor rotations where target errors decrease throughout the adaptation process. Another popular visuomotor paradigm is an invariant error-clamp: experiments where the target error is fixed to a constant value, noncontingent on the participant's movement. In this paradigm, implicit adaptation reaches a ceiling whose value varies somewhere between 15 degrees $^{1}$ and 25 degrees $^{40}$ and does not change with 
rotation size. It is important not to conflate this rotation-invariant saturation, with the implicit saturation phenotype we explored with the competition model in our Neville and Cressman ${ }^{30}$ analysis (Fig. 1G). The ceiling in the invariant error-clamp paradigms appears to be due to an upper bound on implicit corrections ${ }^{40}$. The saturation phenotype in Fig. $1 \mathrm{G}$ is due to implicit competition with explicit strategy.

In invariant error-clamp studies, there is no explicit strategy. In such a case, the competition and independence models are equivalent. However, the models encoded in Eqs. (4) and (5) only describe the standard rotation learning conditions considered in our Results. When there is no explicit strategy, these models predict implicit learning via: $x_{i}^{s s}=b_{i}\left(1-a_{i}+b_{i}\right)^{-1} r$. In an error-clamp study, however, the correct model would be $x_{i}^{s s}=b_{i}\left(1-a_{i}\right)^{-1} r$. These equations differ in their implicit learning gains: $b_{i}\left(1-a_{i}\right)^{-1}$ for constant error-clamp and $b_{i}\left(1-a_{i}+b_{i}\right)^{-1}$ for standard rotations. This has critical implications. For example, in an error-clamp condition, for $a_{i}=0.98$ and $b_{i}=0.3$, the state-space model predicts an implicit steadystate of 15 times the imposed rotation, $r$. In other words, implicit adaptation would need to exceed the rotation size by at least an order of magnitude to reach its steady-state; a $5^{\circ}$ error-clamp would require $75^{\circ}$ of implicit learning to reach a dynamic steady-state and a $30^{\circ}$ rotation would require $450^{\circ}$. In sum, error-clamp rotations require implicit learning that cannot reach the dynamic steady-state described by the state-space model. For these reasons, the steady-states reached in error-clamp studies are likely caused by another mechanism: the ceiling effect shown in Morehead et al. (2017) ${ }^{1}$ and Kim et al. $(2018)^{40}$. However, in a standard rotation, implicit learning must be less than the rotation size (proportional to difference between rotation size and explicit strategy: proportionality constant between 0.6-0.8 in the data sets we consider here). Under these conditions the dynamic steady-state described by the competition model is attainable.

Now, a separate but related question, is what causes the implicit system's upper limit and does it vary across experimental settings. We suspect it does. For example, in Morehead et al. (2017) ${ }^{1}$ the implicit system was limited to $10-15^{\circ}$ learning, but in Kim et al. $(2018)^{40}$ this limit increased to $20-25^{\circ}$. It may be that these limits relate to a reliance on proprioceptive error signals ${ }^{82}$ : implicit learning may be "halted" by some unknown mechanism when the hand deviates too far from the target. This would make sense, as participants are told to move their hand straight to the target and ignore the cursor in this paradigm. In standard rotation paradigms, however, visual errors between the cursor and target may dominate this proprioceptive signal, extending the implicit system's capacity. This might explain why some studies have observed implicit learning levels (e.g., about $35^{\circ}$ in Salomonczyk et al., $2011^{36}$, and even $45^{\circ}$ in Maresch et al., 2021 ${ }^{41}$ ) which greatly exceed the error-clamp limits observed in Morehead et al. and Kim et al.

A critical puzzle that remains, however, is savings. The savings in implicit adaptation observed in Exp. 4 (Fig. 8) contrasts with error-clamp behavior ${ }^{24}$, where implicit learning decreases during the second exposure. We can only speculate why these phenotypes differ. The discrepancy may relate to a divergence in goals. In error-clamp studies, the overall objective is to move straight to the target: to not change one's reach angle. In standard rotation studies, the objective is to move the cursor to the target: to change one's reach angle. This goal could play a role in enhancing or suppressing the implicit system's response; some utility associated with adapting more rapidly may be necessary to obtain savings. On the other hand, responses to visual errors may be suppressed over time during error-clamp, as they are 
1015

1016

1017

1018

1019

1020

1021

1022

1023

1024

1025

1026

1027

1028

1029

1030

1031

1032

1033

1034

1035

1036

1037

1038

1039

1040

1041

1042

1043

1044

1045

1046

1047

1048

1049

1050

1051

1052

1053

1054

1055

1056

irrelevant to the arm's motion. Interestingly, interacting with the visual target in error-clamp does appear to attenuate the implicit response to the rotation ${ }^{10}$.

A second idea relates to the reward system. Sedaghat-nejad et al. (2021) ${ }^{83}$ has shown that saccade adaptation is accelerated when learning improves task success. Learning speeds up when this leads to an increase in reward probability. Adaptation rates are not improved when learning does not impact reward probability. In other words, a higher-level 'desire' to obtain reward may be needed to increase learning rate. Again, such motivation is clear in standard rotation experiments where adaptation will improve task success and reward probability. There is no motivation to adapt more rapidly in error-clamp paradigms; participants are never rewarded. Moreover, as noted above, hitting the target in invariant error-clamp paradigms appears to attenuate the implicit response ${ }^{10}$. Interestingly, a link between reward and savings may be present in the cerebellum. Several studies ${ }^{84}$ have shown that both granule cell layers ${ }^{85}$ and climbing fiber inputs ${ }^{86,87}$ carry reward-related signals to the cerebellum. Thus, it may be that the cerebellum, a potential locus for implicit adaptation ${ }^{6,59-64}$ responds to errors differently when rewards are not attainable $e^{1,24,40}$ such as error-clamp paradigms, versus conventional rotations where more rapid learning promotes reward acquisition. These ideas are speculative and remain to be tested.

Overall, our data suggest that some implicit learning properties may vary across standard rotation and error-clamp paradigms. Considerable future work is needed to better compare these paradigms and test the suppositions outlined above.

\section{The relationship between competition and implicit generalization}

One potential limitation in our analyses relates to implicit generalization. Earlier studies have shown that implicit learning generalizes around the reported aiming direction ${ }^{49,50}$. Thus, participants who aim further away from the target may show smaller implicit adaptation when asked to 'move straight to the target'. While generalization could have contributed to the negative implicit-explicit correlation, its role would be small relative to competition. In earlier studies (Figs. 4B\&C), implicit learning decayed only $5^{\circ}$ or so with $22.5^{\circ}-30^{\circ}$ changes in aiming (Fig. 4-Supplement $2 \mathrm{~B}$ shows $22.5^{\circ}$ re-aiming ${ }^{50}$; Fig. 4-Supplement $2 \mathrm{~A}$ shows $30^{\circ}$ re-aiming ${ }^{49}$ ). However, in Exps. 1-3, we observed between $15^{\circ}-20^{\circ}$ changes in implicit learning (see Figs. 4B\&C) over similar ranges in explicit strategy. Thus, generalization-based decay in implicit learning would need to occur over $300 \%$ more rapidly than earlier reports to match our data.

Critically, in Exps. 1-3 explicit strategy was estimated as total adaptation minus implicit learning. Had generalization reduced the implicit measures, it would falsely inflate our explicit measures. While it is tempting to compare our data in Figs. 2 or $4 \mathrm{~A}-\mathrm{C}$ with past generalization curves, this should not be done without correcting the explicit strategy measures. These corrections revealed that implicit generalization would need to exhibit an implausible narrowing to explain our group-level (e.g., response to stepwise rotation, or instruction) and individual-level results (Appendices 6.1-6.5, Figs. 4C, and 4Supplement 1). Altogether, generalization is not a viable alternative to the competition theory.

Generalization may have played a smaller role in the studies we analyzed, because participants trained with 2 (Tsay et al., 2021), 3 (Exp. 1, Neville \& Cressman, 2018), 4 (Exps. 2-4), 8 (Exp. 5, Maresch et al., 2021), or 12 (Saijo \& Gomi, 2011) targets. Past studies that measured plan-based generalization, only used 1 training target ${ }^{49,50}$ (Fig. 4-Supplements $2 A \& B$ ). Thus, decreases in implicit learning would likely be smaller in our studies, because the generalization curve widens with additional training 
1057

1058

1059

1060

1061

1062

1063

1064

1065

1066

1067

1068

1069

1070

1071

1072

1073

1074

1075

1076

1077

1078

1079

1080

1081

1082

1083

1084

1085

1086

1087

1088

1089

1090

1091

1092

1093

1094

1095

1096

1097

targets $^{47,88}$. For, example, in Neville and Cressman $^{30}$, subjects trained with 3 targets. Given the targets' geometries, 2 had coincided with the neighboring target's aim direction, but 1 did not. A narrow generalization curve would predict a larger aftereffect for the targets that coincided with aim directions, yet no variations in implicit learning were detected across targets (see their supplementary analyses).

Note that unlike past generalization studies ${ }^{49,50}$, we did not use aiming reports to measure explicit strategy ${ }^{49,50}$. We speculate this may play a role in generalization, given that aiming landmarks themselves drive implicit learning ${ }^{8}$ (Fig. 10). For example, past generalization studies observed a discrepancy between exclusion-based implicit learning and report-based implicit learning: the exclusion measures were smaller due to plan-based generalization (Fig. 4-Supplement 2C). But in Exp. 2, the opposite occurred. Exclusion-based implicit learning was larger than implicit learning estimated with reporting (Fig. 4-Supplement 2E). The same phenomenon was noted by Maresch and colleagues ${ }^{41}$ in a condition where reporting was used sparsely during adaptation (Fig. 4-Supplement 2D).

Exp. 1 provided a direct way to test how our data may have been impacted by generalization. In Exp. 1 , a $60^{\circ}$ rotation resulted in $22^{\circ}$ of implicit learning, whereas a $15^{\circ}$ rotation caused about $7^{\circ}$ (Fig. 11 ). Suppose that implicit learning exhibits about $20 \%$ generalization-based decay with a $15^{\circ}$ change in aiming direction as in McDougle et al. $(2017)^{50}$. This decay causes a $(0.2)\left(22^{\circ}\right)=4.4^{\circ}$ decrease in implicit learning in the $60^{\circ}$ rotation, but only a $(0.2)(7)=1.4^{\circ}$ in the $15^{\circ}$ rotation (i.e., $7^{\circ}$ implicit learning). Thus, the absolute change in implicit learning driven by generalization depends on total implicit learning achieved at steady-state, or in Exp. 1, the rotation's size. This is not true in the competition theory: Eq. (4) predicts that the gain relating implicit and explicit adaptation does not depend on rotation size. We tested these diverging predictions in Figs. 4D-F. Critically, behavior matched the competition theory (Fig. $4 F)$. AIC indicated that the competition model better described participant behavior than SPE learning models extended with plan-based generalization (Figs. 4G\&H, Appendix 6.3).

With that said, while the generalization hypothesis did not match important patterns in our data, it remains a very important phenomena that may alter implicit learning measurements. It is imperative that implicit generalization is more thoroughly examined to determine how it varies across experimental methodologies. These data will be needed to accurately evaluate the competitive relationship between implicit and explicit learning.

\section{Error sources that drive implicit adaptation}

Mazzoni and Krakauer ${ }^{2}$ exposed participants to a visuomotor rotation, but also provided instructions for how to re-aim their hand to achieve success. While participants immediately used this strategy to move the cursor through the target, the elimination of task error failed to stop implicit adaptation. These data suggested that the implicit system responded to errors in the predicted sensory consequence of their actions $^{6,89}$, rather than errors in hitting the target.

However, such a model, where implicit systems learn solely based on the angle between aiming direction and the cursor (Eq. (2)), could not account for the implicit-explicit interactions we observed in our data (Figs. 1-5). These interactions could only be described by an implicit error source that is altered by explicit strategy, such as the angle between the cursor and the target (Eq. (1)). For example, in Experiments $2 \& 3$, participants did not aim straight to the target, but rather adjusted their aiming angle by $5-20^{\circ}$ (Fig. 3). These changes in re-aiming appeared to alter implicit adaptation via errors between the 
cursor and the target. This target-cursor error source (Eq. (1)) appeared to provide an accurate account of short-term visuomotor adaptation across a number of studies ${ }^{11,12,26,30-33}$.

We do not mean to suggest, however, that implicit adaptation is solely driven by a single target error. In fact, there are many cases where this idea fails ${ }^{3,8,37}$. We speculate that one feature which alters implicit learning is the simultaneous presence of multiple visual targets. In Figs. 1-9, there was only one visual target on the screen at a time. However, in Mazzoni \& Krakauer (Fig. 10), there were two important visual targets: the adjacent target towards which participants explicitly aimed their hand, and the original target towards which the cursor should move. In theory, the brain could calculate errors with respect to both targets. When we considered the idea that the implicit system adapted to both errors at the same time, we could more completely account for these earlier data (Fig. 10F).

The idea that both kinds of visual error (cursor with respect to the primary target, and cursor with respect to the aimed target) drive implicit learning, could account for other surprising observations. For example, in cases where landmarks are provided to report explicit aiming ${ }^{11,37,49}$, target-cursor error is often rapidly eliminated, but implicit adaptation persists. A dual-error model (Eq. (6)) would explain this continued adaptation based on persistent aim-cursor error. In other words, aiming landmarks may continue to drive adaptation even when primary target errors have been eliminated.

However, the nature of aim-cursor errors remains uncertain. For example, while this error source generates strong adaptation when the aim location coincides with a physical target (Fig. 10I, instruction with target), implicit learning is observed even in the absence of a physical aiming landmark ${ }^{8}$ (Fig. 10I, instruction without target), albeit to a smaller degree. This latter condition may implicate SPE learning that does not require an aiming target. Thus, it may be that the aim-cursor error in Mazzoni and Krakauer is actually an SPE that is enhanced by the presence of a physical target. In this view, implicit learning is driven by a target error module and an SPE module that is enhanced by a visual target error $^{3,10,90}$.

These various implicit learning modules are likely strongly dependent on experimental contexts, in ways we do not yet understand. For example, Taylor \& Ivry $(2011)^{8}$ would suggest that all experiments produce some implicit SPE learning, but less so in paradigms with no aiming targets. Yet, the competition equation accurately matched single-target behavior in Figs. 1-9 without an SPE learning module. It is not clear why SPE learning would be absent in these experiments. One idea may be that the aftereffect observed by Taylor \& Ivry (2011) in the absence of an aiming target, was a lingering associative motor memory that was reinforced by successfully hitting the target during the rotation period. Indeed, such a model-free learning mechanism ${ }^{91}$ should be included in a more complete implicit learning model. It is currently overlooked in error-based systems such as the competition and independence equations.

Another idea is that some SPE learning did occur in the no aiming target experiments we analyzed in Figs. 1-9 but was overshadowed by the implicit system's response to target error. A third possibility is that the SPE learning observed by Taylor \& Ivry (2011) was contextually enhanced by participants implicitly recalling the aiming landmark locations (akin to memory-guided saccade adaptation) provided during the baseline period. This possibility would suggest SPEs vary along a complex spectrum: (1) never providing an aiming target causes little or no SPE learning (as in our experiments), (2) providing an aiming target during past training allows implicit recall that leads to small SPE learning, (3) providing an aiming target that disappears during the movement promotes better recall 
and leads to medium-sized SPE learning (i.e., the disappearing target condition in Taylor \& Ivry), and (4) an aiming target that always remains visible leads to the largest SPE learning levels. This contextdependent SPE hypothesis may be related to recent work suggesting that target errors and SPEs drive implicit learning, but SPEs are altered by distraction ${ }^{54}$.

We speculate that the cerebellum might play an important role in supporting multiple implicit learning modules ${ }^{60,62,92-94}$. Current models propose that complex spikes in Purkinje cells (P-cells) in the cerebellar cortex cause LTD (Marr-Albus-Ito hypothesis). These complex spikes are reliably evoked by olivary input in response to a sensory error ${ }^{93,95,96}$. However, different P-cells are activated by different error directions, thus organizing P-cells into error-specific subpopulations ${ }^{95,96}$. Therefore, our model suggests that two different sources of error might simultaneously transduce learning in two different Pcell subpopulations, which then combine their adapted states into a total implicit correction at the level of the deep nuclei. Thus, errors based on the original target, and the aiming target, might simultaneously activate two implicit learning modules in the cerebellum (Fig. 10H).

Alternatively, it is equally possible that these aim-cursor errors and target-cursor errors engage separate brain regions both inside and outside the cerebellum. In this view, an interesting possibility is that patients with cerebellar disorders ${ }^{6,58,61,97,98}$ may have learning deficits specific to one error but not the other, as recent results suggest ${ }^{62}$. These possibilities remain to be fully tested.

\section{Methods}

1159 Our work involves reevaluation of earlier literature; this includes data from Haith and colleagues $(2015)^{13}$ in Figs. 6 and 8, data from Lerner and Albert et al. $(2020)^{19}$ in Fig. 9, data from Neville and Cressman (2018) ${ }^{30}$ in Figs. 1 and 2, data from Saijo and Gomi (2010) ${ }^{33}$ in Fig. 2-Supplement 3, data from Mazzoni and Krakauer (2006) $)^{2}$ in Fig. 10, data from Taylor and Ivry (2011) in Fig. 10, data from McDougle et al. (2017) $)^{50}$ in Fig. 4, data from Day et al. (2016) ${ }^{49}$ in Fig. 4, data from Tsay et al. (2021) ${ }^{35}$ in Fig. 1, data from Maresch et al. (2021) ${ }^{41}$ in Fig. 5-Supplement 1, and data from Krakauer et al. (2000) ${ }^{47}$ in collected for this work (Exps. 1-5). Note that some methods are described in Appendices 1-8.

\section{Participants}

Here we report the sample sizes used in past studies analyzed here: Haith and colleagues ${ }^{13}(n=14)$, Lerner and Albert et al. ${ }^{19}$ ( $n=16$ for 5 min group, $n=18$ for $24 \mathrm{hr}$ group), Neville and Cressman ${ }^{30}$ (no strategy: $n=11$ for $20^{\circ}, n=10$ for $40^{\circ}, n=10$ for $60^{\circ}$; strategy: $n=10$ for $20^{\circ}, n=11$ for $40^{\circ}, n=10$ for $60^{\circ}$ ), Mazzoni and $\operatorname{Krakauer}^{2}(\mathrm{n}=18)$, Saijo and Gomi ${ }^{33}$ ( $n=9$ for abrupt, $n=9$ for gradual), Maresch et al. ${ }^{41}(n=40$ across the CR, IR-E, and IR-El groups), Tsay et al. ${ }^{35}$ ( $n=25 /$ rotation size), McDougle et al. $(n=15)$, and Taylor and $\operatorname{Ivry}^{8}$ ( $\mathrm{n}=10$ for instruction with visual target, $\mathrm{n}=10$ for instruction without visual target).

All volunteers (ages 18-62) in Experiments 1-5 were neurologically healthy and right-handed. Experiment 1 included $n=36$ participants in the abrupt group (12 Male, 24 Female), $n=37$ participants in the stepwise group ( 6 Male, 30 Female, 1 opted to not report). Experiment 2 included $n=9$ participants (5 Male, 4 Female) in the No PT-Limit group and included $n=13$ participants ( 6 Male, 7 Female) in the PTLimit group. Experiment 3 included $n=35$ participants in the No PT-Limit group (7 Male, 14 Female), $n=21$ participants in the Limit PT group ( 20 Male, 15 Female), and $n=12$ ( 5 Male, 7 Female) participants in the decay-only group. Experiment 4 included $n=10$ participants ( 6 Male, 4 Female). Experiment 5 included 
$\mathrm{n}=20$ participants (10 Male, 10 Female) with $\mathrm{n}=9$ in the 5 min group and $\mathrm{n}=11$ in the 24 group. Experiment 1 was approved by the York Human Participants Review Sub-committee. Experiments 2-5 were approved by the Institutional Review Board at the Johns Hopkins School of Medicine.

\section{Data extraction}

When acquiring data from published figures we first attempted to open it in Adobe Illustrator. Depending on how these figures were saved and embedded, occasionally the figure could be decomposed into its layers. This allowed us to extract the $x$ and $y$ pixel values for each data point (which appeared as an object) to interpolate the necessary data from the figure. However, in some cases, objects and layers could not be obtained in Illustrator. In these cases, we used the utility GRABIT in MATLAB to extract the necessary data. We clearly indicate which approach was used when discussing each dataset below. Note that the authors provided source data for our Maresch et al., $2021^{41}$ and Tsay et al., $2021^{35}$ analyses.

\section{Apparatus}

In Experiments 1, 2, 4, and 5 participants held the handle of a robotic arm and made reaching movements to different target locations in the horizontal plane. The forearm was obscured from view by an opaque screen. An overhead projector displayed a small white cursor (diameter $=3 \mathrm{~mm}$ ) on the screen that tracked the hand's motion. We recorded the position of the handle at submillimeter precision with a differential encoder. Data were recorded at $200 \mathrm{~Hz}$. Protocol details were similar for Haith and colleagues $^{13}$, Neville and Cressman ${ }^{30}$, Saijo and $\mathrm{Gomi}^{33}$, and Maresch et al. ${ }^{41}$ in that participants gripped a two-link robotic manipulandum, were prevented from viewing their arm, and received visual feedback of their hand position in the form of a visual cursor. In Lerner and Albert et al. ${ }^{19}$, participants performed pointing movements with their thumb and index finger while gripping a joystick with their right hand. In Mazzoni and Krakauer $^{2}$, participants rotated their hand to displace an infrared marker placed on the index finger. In Taylor and Ivry ${ }^{8}$, hand position was tracked via a sensor attached to the index finger while participants made horizontal reaching movements along the surface of a table. In Day et al. $(2016)^{49}$, Krakauer et al. (2000) ${ }^{47}$, and McDougle et al. $(2017)^{50}$, participants moved a stylus over a digitizing tablet. In Experiment 3, participants were tested remotely on a personal computer. They moved a cursor on the screen by sliding their index finger along the track pad. These conditions were similar in Tsay et al $(2021)^{35}$.

\section{Visuomotor rotation}

1215 Experiments 1-5 followed a similar protocol. At the start of each trial, the participant brought their hand 1216 to a center starting position (circle with $1 \mathrm{~cm}$ diameter). After maintaining the hand within the start 1217 circle, a target circle $\left(1 \mathrm{~cm}\right.$ diameter) appeared in 1 of 4 positions $\left(0^{\circ}, 90^{\circ}, 180^{\circ}\right.$, and $\left.270^{\circ}\right)$ at a 1218 displacement of $8 \mathrm{~cm}$ (Experiments 2, 4, and 5). In Experiment 5, 8 targets were used, spaced in 1219 increments of $45^{\circ}$. In Experiment 1, 3 targets were used positioned in a triangular wedge $\left(45^{\circ}, 90^{\circ}\right.$, and $1220135^{\circ}$ ). Participants made a brisk movement that terminated on (Exp. 1) or moved through (Exps. 2-5) the 1221 target. Each experiment consisted of epochs of 4 trials ( 3 trials for Experiment 1, 8 trials for Experiment

1222 5) where each target was visited once in a pseudorandom order. 
Participants were provided audiovisual feedback about their movement speed and accuracy. If a movement was too fast (duration $<75 \mathrm{~ms}$ ) or too slow (duration $>325 \mathrm{~ms}$ ) the target turned red or blue, respectively. If the movement was the correct speed, but the cursor missed the target, the target turned white. Successful movements were rewarded with a point (total score displayed on-screen), an onscreen animation, and a pleasing tone $(1000 \mathrm{~Hz})$. If the movement was unsuccessful, no point was awarded, and a negative tone was played $(200 \mathrm{~Hz})$. Participants were instructed to obtain as many points as possible throughout the experimental session. Experiment 1 was similar but used $10 \mathrm{~cm}$ reach displacements and had no upper bound on movement duration.

Once the hand reached the target, visual cursor feedback was removed, and a yellow marker

1231

1232

1233

1234

1235

1236

1237

1238

1239

1240

1241

1242

1243

1244

1245

1246

1247

1248

1249

1250

1251

1252

1253

1254

1255

1256

1257

1258

1259

1260

1261

1262

1263

As a measure of adaptation, we analyzed the reach angle on each trial. The reach angle was was frozen on-screen to indicate the final hand position. At this point, participants were instructed to move their hand back to the starting position (in Exp. 1, this return movement was aided by a circle centered on the start position, whose radius matched the hand's displacement). The cursor remained hidden until the hand was moved within $2 \mathrm{~cm}$ of the starting circle $(1 \mathrm{~cm}$ in Exp. 1).

Movements were performed in one of three conditions: null trials, rotation trials, and no feedback trials. On null trials, veridical feedback of hand position was provided. On rotation trials, the on-screen cursor was rotated relative to the start position. On no feedback trials, the subject cursor was hidden during the entire trial. No feedback was given regarding movement endpoint, accuracy, or timing. measured as the angle between the hand and the target (relative to the start position), at the moment where the hand exceeded $95 \%$ of the target displacement. In Experiment 1, reach angles were measured at the hand's maximum velocity.

Experiments in Haith et al. $(2015)^{13}$, Lerner \& Albert et al. (2020) ${ }^{19}$, McDougle et al. (2017), Taylor and Ivry $(2011)^{8}$, Neville and Cressman $(2018)^{30}$, Saijo and Gomi $(2010)^{33}$, Day et al. $(2016)^{49}$, McDougle et al. $(2017)^{50}$, Krakauer et al. (2000) ${ }^{47}$, Maresch et al. $(2021)^{41}$, Tsay et al. $(2021)^{35}$, and Mazzoni \& Krakauer $^{2}$ were collected using similar, but separate protocols. Important differences between these studies and the rotation protocol mentioned above are briefly described in the sections below.

\section{Statistics}

Parametric t-tests were performed in MATLAB R2018a. For these tests, we report the t-statistic, p-value, and Cohen's $d$ as a measure of effect size. A repeated measures ANOVA (rm-ANOVA) was used to measure differences in prediction error in Fig. 4E. Two-way repeated measures ANOVAs were used in Figs. 6B-D to measure how preparation time (low vs high) and exposure number (Day 1 vs. Day 2) altered learning rate, reach angle, and model-based error sensitivity measurements, respectively. Mixed-ANOVAs were used in Fig. $8 \mathrm{C}$ to examine how learning (both rate and mean over initial trials) was altered by preparation time conditions (between-subject factor: Haith et al. ${ }^{13}$ vs Experiment 4) and exposure number (within-subjects factor, exposure 1 vs exposure 2). A two-way ANOVA was used in Fig. $9 \mathrm{C}$ to determine how interference patterns changed with movement preparation time (no limit vs limit) and time passage ( $5 \mathrm{~min}$ vs $24 \mathrm{hr}$ ). For all two-way and mixed-ANOVAs, we initially determined whether there was a statistically significant interaction effect between each factor. In cases where this 
interaction effect was statistically significant, we next measured simple main effects via one-way ANOVA.

\section{Competition Map}

In Fig. 7 we created a competition map to describe the interactions between explicit strategy and implicit learning predicted by the competition theory. To generate this map, we used a state-space model (Eqs. (1-3)) where implicit learning and explicit learning were both driven by target errors:

$$
\begin{aligned}
& x_{i}^{(n+1)}=a_{i} x_{i}^{(n)}+b_{i} e^{(n)} \\
& x_{e}^{(n+1)}=a_{e} x_{e}^{(n)}+b_{e} e^{(n)}
\end{aligned}
$$

1272

1273

1274

1275

1276

1277

1278

1279

1280

1281

1282

1283

1284

1285

1286

1287

1288

1289

1290

1291

The terms $a_{i}$ and $a_{e}$ represent implicit retention and explicit retention. The terms $b_{i}$ and $b_{e}$ represent implicit error sensitivity and explicit error sensitivity.

Because implicit and explicit systems share a common error source in this target error model, their responses will exhibit competition. That is, increases in explicit adaptation will necessarily be coupled to decreases in implicit adaptation. To summarize this interaction, we created a competition map. The competition map describes common scenarios in which the goal is to compare two different learning curves. For example, one might want to compare the response to a $30^{\circ}$ visuomotor rotation under two different experimental conditions. Another example would be savings, where we compare adaptation to the same perturbation at two different timepoints. In these cases, it is common to measure the amount of implicit and explicit adaptation, and then compare these across conditions or timepoints.

The critical point is that changes in the amount of implicit adaptation reflect the modulation of both implicit and explicit responses to error. This competition will occur at all points during the adaptation timecourse (Appendix 1), but is easiest to mathematically validate at steady-state. As described in the main text, the steady-state level of implicit adaptation can be derived from Eqs. (1-3). This derivation resulted in the competition equation shown in Eq. (4). Note that Eq. (4) predicts the steady-state level of implicit learning from the implicit retention factor, implicit error sensitivity, mean of the perturbation, and critically, the steady-state explicit strategy. If the explicit system is also described using a state-space model as in Eq. (7), it can be shown that Eq. (4) can be equivalently expressed in terms of the implicit and explicit learning parameters according to Eq. (8):

$$
x_{i}^{s s}=\frac{b_{i}\left(1-a_{e}\right)}{\left(1-a_{i}+b_{i}\right)\left(1-a_{e}+b_{e}\right)-b_{i} b_{e}} r
$$

Eq. (8) provides the total amount of implicit adaptation as a function of the retention factors, $a_{i}$ and $a_{e}$, as well as the error sensitivities, $b_{i}$ and $b_{e}$. We used Eq. (8) to construct the competition map in Fig. 7A, by comparing the total amount of implicit learning across a reference condition and a test condition.

For our reference condition, we fit our state space model to the mean behavior in Haith et al. ${ }^{13}$ (Fig. 6B, Day 1, left). This model best described adaptation during the first perturbation exposure using the parameter set: $a_{i}=0.9829, a_{e}=0.9278, b_{i}=0.0629, b_{e}=0.0632$. Next, we imagined that implicit error sensitivity and explicit error sensitivity differed across the reference and test conditions. On the $x$-axis of the map, we show a percent change in $b_{i}$ from the reference condition to the test condition. On the $y-$ axis of the map, we show a percent change in $b_{e}$ from the reference condition to the test condition. The retention factors were held constant across conditions. Then for each condition we calculated the total 
amount of implicit learning using Eq. (8). The color at each point in the map represents the percent change in the total amount of implicit learning from the reference condition to the test condition.

As described in the main text, the competition map (Fig. 7A) is composed of several important regions (Fig. 7B). In Region A, there is a decrease in implicit error sensitivity (from reference to test) as well as a decrease in the total amount of implicit adaptation predicted by Eq. (8). In Region B, Eq. (8) predicts a decrease in implicit adaptation, despite an increase in implicit error sensitivity. In Region $D$, there is an increase both in implicit error sensitivity as well as steady-state implicit learning. In Region E, there is an increase in implicit adaptation, despite a decrease in implicit error sensitivity. Finally, Region $C$ shows cases where there are changes in implicit error sensitivity, but the total absolute change in implicit adaptation (Eq. (8)) is less than $5 \%$. To localize this region, we solved for the linear bounds that describe a $5 \%$ increase or a $5 \%$ decrease in the output of Eq. (8).

Neville \& Cressman (2018) 30

To understand how enhancing explicit strategy might alter implicit learning, we considered data collected by Neville and Cressman ${ }^{30}$. Here the authors tested how awareness of a visuomotor rotation altered the adaptation process. To do this, participants $(n=63)$ were divided into several groups. In the instructed groups (Fig. 2A, purple), participants were instructed about the rotation and a compensatory strategy prior to perturbation onset. In other groups, no instruction was provided (Fig. 1C; Fig. 2A, black). During rotation periods, participants reached to three potential targets. Implicit contributions to behavior were measured at 4 different periods using "exclusion" trials. During exclusion trials, the authors instructed participants to reach (without visual feedback) as they did during the baseline period prior to perturbation onset (without using any knowledge of the perturbation gained thus far). Exclusion trial reach angles served as our implicit learning measure. The difference between total adaptation and exclusion trial reach angles served as our explicit learning measure.

At the start of the experiment all participants performed a baseline period without a rotation for 30 trials. Baseline implicit and explicit reach angles were then assayed. At this point, participants in the strategy group were briefed about the perturbation with an image that depicted how feedback would be rotated, and how they could compensate for it. Then all groups were exposed to the first block of a visuomotor rotation for 30 trials. Some participants experienced a $20^{\circ}$ rotation, others a $40^{\circ}$ rotation, and others a $60^{\circ}$ rotation. After this first block, implicit and explicit learning were assayed. This block structure was repeated two more times.

Here we focused on implicit and explicit adaptation measures obtained at the end of the final block. To obtain these data, we extracted the mean participant response and the associated standard error of the mean, directly from the primary figures reported by Neville and Cressman ${ }^{30}$ using Adobe Illustrator CS6. The implicit and explicit responses in all 6 groups are shown in Fig. 2-Supplement 1. The marginal effect of instruction (average over rotation sizes) is shown in Figs. 2B and 2C.

Finally, we tested whether the competition equation (Eq. (4)) or independence equation (Eq. (5)) could account for the levels of implicit learning observed across rotation magnitude and awareness conditions. To do this, we used a bootstrapping approach. Using the mean and standard deviation obtained from the primary figures, we sampled hypothetical explicit and implicit aftereffects for 10 participants. We then calculated the mean across these 10 simulated participants. After this, we used 
fmincon in MATLAB R2018a to find an implicit error sensitivity that minimized the following cost 1345 function:

$$
\theta_{\text {fit }}=\underset{\theta}{\operatorname{argmin}} \sum_{n=1}^{6}\left(x_{i_{n}}^{s s}-\hat{x}_{i_{n}}^{s s}\right)^{2}
$$

This cost function represents the difference between the simulated level of implicit adaptation, and the amount of implicit learning that would be predicted for a given perturbation size and simulated explicit adaptation, according to our competition framework (Eq. (4)) or independence framework (Eq. (5)). For this process, we set the implicit retention factor to 0.9565 (see Measuring properties of implicit learning). Therefore, only the implicit error sensitivity remained as a free parameter. In sum, we aimed to determine if a single implicit error sensitivity could account for the amount of adaptation across the no instruction group, instruction group, and each of the 3 perturbation magnitudes $\left(20,40\right.$, and $\left.60^{\circ}\right)$. The combination of instruction and perturbation magnitude yielded 6 groups, hence the upper limit on the sum in Eq. (9). We repeated this process for a total of 10,000 simulated groups.

In Figs. 2B and 2C, we show the marginal effect of instruction on the implicit aftereffect. This was obtained by averaging across each of the 3 rotation magnitudes shown in Fig. 2-Supplement 1, for each model. In Fig. 1, we show the implicit learning levels predicted by the model across all rotation sizes in the no-instruction group. Model predictions across all rotations sizes in the instruction group are shown in Fig. 2-Supplement 1. Again, all model predictions were made using the same underlying implicit learning parameter set.

\section{Experiment 1}

To examine how changes in rotation onset and magnitude altered implicit learning, we recruited two participant groups. In the abrupt group, subjects $(n=36)$ experienced a $60^{\circ}$ visuomotor rotation abruptly. In the stepwise group, subjects $(n=37)$ experienced 4 separate rotation magnitudes in sequence: $15^{\circ}$, $30^{\circ}, 45^{\circ}$, and $60^{\circ}$. Thus, the experiment had 4 learning periods, one for each rotation size. Each period lasted 66 trials, over which 3 targets $\left(45^{\circ}, 90^{\circ}, 135^{\circ}\right)$ were visited 22 times. This same structure was used in the abrupt group, though the rotation magnitude remained constant over each learning block. Twice during each block (about 75\% into each block and again at the end), exclusion trials were used to measure implicit adaptation. On these trials, subjects were told to stop using explicit strategies and to reach as they had during the baseline period. The average exclusion trial reach angle (across both probe periods in each block) served as our implicit learning measure. The difference between total adaptation and the average exclusion trial reach angle served as our explicit learning measure. Total adaptation was calculated as the average reach angle on the last 20 trials in each learning period.

Here we focused on implicit and explicit adaptation measures obtained during each block. These measures are shown in Figs. $1 \mathrm{I}$ and 2J. In Figs. 1 and 2, we tested how well these measures were predicted by the competition and independence equations. The same model parameters were used in equation can be written as $x_{i}^{s s}=p_{i}\left(r-x_{e}^{s s}\right)$ and the independence equation can be written as $x_{i}^{s s}=p_{i} r$, where $p_{i}$ is a scalar gain determined by $a_{i}$ and $b_{i}$. Thus, the gain $p_{i}$ is the only unknown model parameter.

Our goal was to identify one gain (one for each model) that could parsimoniously explain

1383 behavior across the stepwise and abrupt groups. Thus, we identified the optimal gain that minimized the 
squared error between the model predictions and implicit adaptation across 5 measures: $15^{\circ}$ stepwise learning, $30^{\circ}$ stepwise learning, $45^{\circ}$ stepwise learning, $60^{\circ}$ stepwise learning, and $60^{\circ}$ abrupt learning. For the abrupt condition, we did not observe a statistically significant difference in implicit aftereffect across the 4 learning periods ( $r$-ANOVA, $F(3,105)=2.21, p=0.091, \eta_{p}{ }^{2}=0.059$ ); thus, we averaged across learning periods to obtain a single implicit measure. We then identified the $p_{i}$ parameter that minimized squared error according to Eq. (9), with all 5 terms described above appearing in the sum.

To construct the model predictions shown in Figs. 1 and 2, we used a bootstrapping approach. Participants in the stepwise and abrupt group were resampled with replacement 1000 times. Each time the average implicit learning measure was calculated across the 5 conditions described above. Each model was then fit to these average data. Thus, Figs. 1 and 2 show the mean implicit learning predicted by each model across all 1000 iterations, as well as the associated standard deviation.

In the main text, we also report a statistical comparison between implicit learning predicted by the competition theory in the $60^{\circ}$ stepwise and $60^{\circ}$ abrupt conditions. This statistic was obtained using a different procedure. Here the optimal $p_{i}$ was determined again using Eq. (9), but without bootstrapping. Average across-subject implicit adaptation in the $15^{\circ}$ stepwise period, $30^{\circ}$ stepwise period, $45^{\circ}$ stepwise period, $60^{\circ}$ stepwise period, and $60^{\circ}$ abrupt period appeared within the sum in Eq. (9). Then implicit learning was predicted using Eq. (4) assuming that each participant had the same $p_{i}$ learning gain. We then conducted a paired t-test between $60^{\circ}$ stepwise and $60^{\circ}$ abrupt implicit learning predicted by the model.

Exp. 1 was used extensively to compare the competition theory with an SPE generalization model.

All details concerning this analysis are provided in Appendix 6. Results are depicted in Fig. 4.

Finally, we analyzed subject-to-subject pairwise relationships between implicit learning, explicit strategy, and total adaptation (average over last 40 rotation trials) in Fig. 5-Supplements $1 B$, E, and $H$. For these analyses, we combined subjects across the $60^{\circ}$ rotation period in the abrupt and stepwise groups. Note we excluded 3 outlying participants whose reach angles differed by more than 3 median absolute deviations from the total population on at least $33 \%$ of all trials. This yielded a total dataset of $n=70$. To analyze each pairwise relationship, we used linear regressions. In addition, we analyzed the same relationships during the $30^{\circ}$ rotation period in the stepwise group (Fig. 5-Supplements $2 \mathrm{~A}$ and 3B).

Tsay et al. $(2021)^{35}$

To evaluate the competition and independence models, we analyzed how implicit and explicit systems responded to rotation sizes between $15^{\circ}$ and $90^{\circ}$ in experiments conducted by Tsay and colleagues ${ }^{35}$. Data in these experiments was collected remotely via a laptop-based experiment. Participants moved to targets at $45^{\circ}$ and $135^{\circ}$, which alternated across trials. Participants were exposed to a $15^{\circ}, 30^{\circ}, 60^{\circ}$, or $90^{\circ}$ rotation ( $n=25 /$ rotation size). The reach angles during an initial baseline period, rotation period, and terminal no aiming period are shown in Fig. 1M. During the no aiming period, participants reached to each target 10 times ( 20 trials total). To calculate implicit learning (Fig. 1M, no aiming; Fig. 1N, Fig. 1Q, data) we averaged the reach angle across the 20 no aiming trials. To calculate total adaptation, we measured the average reach angle over the last 40 reaching trials (Fig. 5-Supplements $1 \mathrm{C}, 1 \mathrm{~F}, 2 \mathrm{~A}$, and 3A). To calculate explicit strategy, we computed the difference between total adaptation and implicit learning (Fig. 10). We also calculated the implicit driving input in the competition theory (rotation minus 
explicit strategy) in Fig. 1P. We also reported an explicit gain in the main text. This gain was calculated by dividing the difference between explicit strategies by the difference in rotation sizes corresponding to each strategy (and then multiplying by 100 to obtain a percentage).

To investigate the non-monotonic relationship between implicit learning and rotation size (Fig. $1 \mathrm{~N})$, we used the competition and independence models. In Fig. 1Q, we fit each model to the measured data. To do this, we estimated the implicit retention factor using the reach angle decay rate during the terminal no aiming period (see Measuring properties of implicit learning, estimate $=0.974$ ). Next, we used a least-squares approach to determine the optimal implicit error sensitivity $\left(b_{i}\right)$ that best matched the implicit reach angles measured across all 4 rotation sizes. Note that since $a_{i}$ and $b_{i}$ appear together in the implicit learning gain, $p_{i}$, fitting the gain directly would produce the same results.

For the competition theory, we averaged the implicit and explicit responses within each rotation group, and then identified the $b_{i}$ value that best predicted implicit learning across rotation sizes according to the competition equation (Eq. (4)). To do this, we used the fminbnd utility in MATLAB R2018a. This yielded $b_{i}=0.0319$. We then used the same $a_{i}$ and $b_{i}$ parameter values to predict total implicit learning across all 4 rotation sizes via Eq. (4), assuming all participants had the same implicit learning parameters (Fig. 1Q, competition). Again, this is equivalent to directly fitting the implicit learning gain $p_{i}$.

We used a bootstrapping procedure to identify the optimal $b_{i}$ parameter in the independence model. To do this, we sampled participants in each rotation group with replacement 10,000 times. Each time, we calculated the average implicit response, and then minimized the squared error (fminbnd in MATLAB R2018a) between this implicit response and that predicted by the independence model (Eq. (5)), across all 4 rotations sizes. We used the $a_{i}$ and $b_{i}$ estimated in the bootstrapping procedure to predict total implicit learning according to the independence model (Fig. 1Q, independence).

Finally, we analyzed subject-to-subject pairwise relationships between implicit learning, explicit strategy, and total adaptation in Fig. 5-Supplements 1C, F, and I. For this, we considered participants in the $60^{\circ}$ rotation group. To analyze each relationship, we used linear regressions. We also analyzed these same relationships during the $30^{\circ}$ rotation period (Fig. 5-Supplements $2 \mathrm{~A}$ and $3 \mathrm{~A}$ ).

Note that Tsay et al. also tested participants in an invariant error-clamp experiment. We did not analyze these data here for two reasons. First, no strategy is used in invariant error-clamp paradigms. This means that SPE and target errors are the same, meaning that the competition model and independence model cannot be distinguished (they make the same predictions). Second, as described in our Discussion (see the section on invariant error-clamp learning), the competition and independence models derived in Eqs. (4) and (5) only apply to standard rotation learning. The implicit learning gain in the invariant error-clamp paradigm is not the same and predicts implicit learning levels that cannot be physically achieved (see Discussion).

\section{Experiment 2}

1463 To test whether changes in explicit strategy altered implicit learning at the individual-level, we tested 1464 two adaptation conditions. In the first experiment, participants adapted to a visuomotor rotation without any limits applied to preparation time (No PT Limit), thus allowing participants to use explicit strategy. In a second experiment, we strictly limited preparation time in order to suppress explicit strategy (Limit PT). 
Participants in the No PT Limit condition began with 10 epochs of null trials ( 1 epoch $=4$ trials), followed by a rotation period of 60 epochs. Other details concerning the experiment paradigm are described in Visuomotor rotation. At the end of the perturbation period, we measured the amount of implicit and explicit learning. To do this, participants were instructed to forget about the cursor and instead move their hand through the target without applying any strategy to compensate for the perturbation. Furthermore, visual feedback was completely removed during these trials. All 4 targets were tested in a randomized sequence. To quantify the total amount of implicit learning, we averaged the reach angle across all targets (Fig. $3 B \& G$ ). To calculate the amount of explicit adaptation, we subtracted this measure of implicit learning from the mean reach angle measured over the last 10 epochs of the perturbation prior to the verbal instruction (results did not change whether we used 5, 10, 15 or 20 epochs to calculate total learning). Explicit measures are shown in Fig. 3G and 3N (E2).

In the Limit PT group, we suppressed explicit adaptation for the duration of the experiment by limiting the time participants had to prepare their movements. To enforce this, we limited the amount of time available for the participants to start their movement after the target location was shown. This upper bound on reaction time was set to $225 \mathrm{~ms}$ (we corrected reaction times by the average screen delay, $55 \mathrm{~ms}$ ). If the reaction time of the participant exceeded the desired upper bound, the participant was punished with a screen timeout after providing feedback of the movement endpoint. In addition, a low unpleasant tone $(200 \mathrm{~Hz})$ was played. This condition was effective in limiting reaction time (Fig. 3F). This experiment started with 10 epochs ( 1 epoch $=4$ trials) of null trials. After this, the visuomotor rotation was introduced for 60 epochs. At the end of the perturbation period, we measured retention of the visuomotor memory in a series of 15 epochs of no feedback trials (Fig. 3E, no feedback).

Our goal was to test whether the putative implicit learning properties measured in the Limit PT group could be used to predict the subject-to-subject relationship between implicit and explicit adaptation in the No PT-Limit group (according to Eq. (4)). To do this, we measured each participant's implicit retention factor and error sensitivity in the Limit PT condition (see Measuring properties of implicit learning below). We then averaged each parameter across participants. Next, we inserted these mean parameters into Eq. (4). With these variables specified, Eq. (4) predicted a specific linear relationship between implicit and explicit learning (Fig. 3G, model). We overlaid this prediction on the actual amounts of implicit and explicit adaptation measured in each No PT-Limit participant (Fig. 3G, black dots). We performed a linear regression across these measured data (Fig. 3G, black line, measured). We report the slope and intercept of this regression as well as the corresponding $95 \%$ confidence intervals.

Lastly, we also asked participants to verbally report their explicit strategy. After the implicit probe trials, we showed each target once again, with a ring of small white landmarks placed at an equal radial distance around the screen ${ }^{11}$. A total of 108 landmarks was used to uniformly cover the circle. Each landmark was labeled with an alphanumeric string. Subjects were asked to report the nearest landmark that they were aiming towards at the end of the experiment in order to move the cursor through the target when the rotation was on. The mean angle reported across all 4 targets was calculated to provide an additional assay of explicit adaptation. However, several (25\% across all participants and trials) reports appeared inaccurate in that they had the incorrect sign (participants 
1509

1510

1511

1512

1513

1514

1515

1516

1517

1518

1519

1520

1521

1522

1523

1524

1525

1526

1527

1528

1529

1530

1531

1532

1533

1534

1535

1536

1537

1538

1539

1540

1541

1542

1543

1544

1545

1546

1547

1548

1549

sign errors ${ }^{44}$ (errors of same magnitude, opposite sign), we took each report's absolute value when calculating explicit recalibration.

Next, we calculated a report-based implicit measure by subtracting report-based explicit strategy from total adaptation. While report-based implicit learning was smaller than reach-based implicit learning (Fig. 3-Supplement 2B), and report-based explicit strategy was larger than reach-based strategy (Fig. 3-Supplement 2A), the two exhibited close correspondence with Eq. (4) (Fig. 3-Supplement 2C).

Lastly, we also analyzed our reach-based implicit and explicit learning measures in a generalization analysis (Figs. 5A\&B). This analysis is described in Appendix 6.

\section{Experiment 3}

We remotely tested 3 participant groups (No PT Limit, Limit PT, and decay-only). Participants controlled a cursor by moving their index finger across the track pad of their personal computer. The experiment was coded in Java. To familiarize themselves with the task, participants watched a 3-minute instructional video. In this video, the trial structure, point system, and feedback structure were described. After this video, there was a practice period. During the practice period, the software tracked the participant's reach angle on each trial. If the participant achieved success on fewer than $65 \%$ of trials (measured based on an angular target-cursor discrepancy $\leq 30^{\circ}$, reaction time $\leq 1 \mathrm{sec}$, and movement duration $\leq$ $0.6 \mathrm{sec}$ ), they had to re-watch the instructional video and re-do the practice period. Movements were brisk and straight, as in standard in-person rotation studies (two example participants are shown in the No PT Limit and Limit PT groups in Fig. 3-Supplement 3).

After the practice period ended, the testing period began. This testing period was similar to the No PT Limit condition in Experiment 2. On each trial, participants reached to 1 of 4 targets (up, down, left, and right). Each target was visited once pseudorandomly in a cycle of 4 targets. After an initial 10cycle null period, a $30^{\circ}$ visuomotor rotation was imposed that lasted for 60 epochs. At the end of the rotation period, we measured implicit and explicit adaptation. The experiment briefly paused, and an audiovisual recording was played that instructed participants to not use any strategy and to move their hand straight through the target. After the experiment resumed, feedback was removed, and participants performed 20 cycles of no-feedback probe trials. In the No PT-Limit group, participants were told to stop aiming on these no-feedback trials, and to move their hand straight to the target.

We measured subject-to-subject correlations between implicit and explicit adaptation in the No PT Limit group. For this, we calculated two implicit learning measures. The early implicit aftereffect was simply the aftereffect observed on the first no-aiming, no-feedback probe cycle (Fig. 3Q). The late implicit aftereffect was the average aftereffect observed on the last 15 cycles of this no-aiming, nofeedback period (Fig. 3P). To measure explicit learning, we calculated the difference between the total amount of adaptation (mean reach angle over last 10 cycles of the rotation period) and the first cycle of the no-aiming, no-feedback period. We investigated the relationship between explicit adaptation and the early and late implicit aftereffects via linear regression in Figs. 3Q and 3P, respectively. For the early implicit aftereffect, we measured the $95 \% \mathrm{Cl}$ for the slope and intercept. Note that explicit learning measures are also reported in Fig. $3 \mathrm{~N}$ (E3, black) and late implicit learning measures are reported in Fig. 30 (No Lim.). 
In addition, we also analyzed the relationship between total adaptation and implicit and explicit adaptation in the No PT Limit group. As described in the main text, the competition theory predicted that total adaptation and explicit strategy should have a positive relationship, whereas total adaptation and implicit strategy should have a negative relationship (see Appendix 7). In Fig. 5G, we show the relationship between total adaptation and the explicit learning measure. In Fig. $5 \mathrm{H}$, we show the relationship between total adaptation and the late implicit learning measure. The brown lines denote a linear regression across individual participants.

Finally, we also considered the No PT Limit data in our generalization analyses in Figs. 4A\&C. This process was the same as for Experiment 2 as shown in Fig. 4A\&B. See Appendix 6.

Next, we also tested a Limit PT group in Exp. 3. Here we attempted to suppress explicit strategies by limiting movement preparation time. To determine the limiting preparation time, we used an adaptive algorithm during the baseline period to decrease or increase the preparation time limit in response to a correct or incorrect reach responses (i.e., reaches to the correct or incorrect target). This limit was capped at $350 \mathrm{~ms}$, but this upper bound did not include screen delay. We used audiovisual feedback throughout the experiment to enforce the preparation time limit. If the reaction time of the participant exceeded the desired upper bound, the participant was played a low-pitched tone during which the screen briefly timed out and shown a message to "react faster". This condition produced the preparation times shown in Fig. 3M. Apart from this, the experiment protocol was the same as the No PT Limit group.

To test whether limiting preparation time was successful in inhibiting explicit strategy, we calculated explicit strategy as in the No PT Limit. Explicit strategies were dramatically inhibited by limiting preparation time (Fig. 30, E3, red). Second, we wanted to measure implicit learning properties in the Limit PT condition and use these to predict the implicit-explicit relationship in the No PT Limit group, with the competition theory. For the latter, we used the same method described above for Experiment 2 (also see Measuring properties of implicit learning). Using the Limit PT data, the competition theory predicted the line shown in blue in Fig. 3Q. The black data points show the implicit and explicit learning measures in the No PT Limit group. Also note that consistent with the competition theory, limiting preparation time led to an increase in implicit learning (Fig. 30, PT-Limit).

As stated above, in the No PT Limit and Limit PT groups, participants were instructed to stop reaiming during the no feedback period, and to move their hand straight to the target (Figs. 3I\&L, no aiming). We used the voluntary change in reach angle to estimate explicit strategy. However, the instruction period lasted about 30 seconds, which may have caused decay in temporally-labile implicit learning ${ }^{30,41,72}$. To measure how much implicit learning had decayed over this time delay, we varied the instruction condition in a decay-only group $(n=12)$. The decay-only group adapted using the same restricted reaction time paradigm as the Limit PT group. However, prior to the no feedback period, participants were told that the disturbance between the cursor and their movement would still be present when they returned to the experiment, but they would no longer be able to see the cursor. Still, they were told to imagine this disturbance and to try and move the imagined cursor to the target. Changes in reach angle in this group, would be due solely to decay in implicit learning (Fig. 8Supplement 1). We compared the behavior in the decay-only group to the Limit PT group in Fig. 8Supplement 1. 
Finally, we used a separate procedure to estimate screen delay. To do this, participants were told to tap a circle that flashed on and off in a regular, repeating cycle. Participants were told to predict the appearance of the circle, and to tap exactly as the circle appeared. Because the stimulus was predictable, the difference between the appearance time, and the participant's button press, revealed the system's visual delay. The average visual delay we measured was $154 \mathrm{~ms}$. This average value was subtracted out in the preparation times reported in Figs. 3J\&M, as well as Fig. 8-Supplement 1. rotation at a single target. On each trial they reported their aiming direction, using a ring of visual landmarks. This study measured implicit generalization, by instructing participants to aim towards untrained targets. We reproduce this curve in Fig. 4A (Day 1T). We only show the curve starting at the average aiming direction $\left(0^{\circ}\right.$ on the $x$-axis), towards the training target direction (i.e., in the direction participants will change their aim when instructed to aim to the primary target). Note in Figs. 4B and 4C, only the initial two points along the curve are shown.

Last, we also compared implicit learning measured across 2 groups reported in their Fig. 2 . In the "target" group in Fig. 4-Supplement 2A, implicit aftereffects were periodically probed at the trained target location, by asking subjects to reach to the target without aiming. In the "aim" group, implicit aftereffects were probed at a target location $30^{\circ}$ away from the trained target, consistent with the direction of the most frequently reported aim. In Fig. 4-Supplement 2A, we show the implicit aftereffect measured on the first aftereffect trial at the end of the experiment. In Fig. 4-Supplement $2 \mathrm{C}$ we again show the implicit aftereffect measured at the trained target location in the "probe" condition. The "report" condition shows the amount of implicit learning estimated by subtracting the reported explicit strategy from the reported reach angle on the last cycle of the rotation. Note that all data were extracted using the primary source's figures with MATLAB's GRABIT utility.

Fig. 4A reproduces generalization curves measured in Krakauer et al. We extracted curves shown in Fig. 7B in Krakauer et al. (2000) ${ }^{47}$ using GRABIT in MATLAB R2018a. To demonstrate how generalization curves are altered by the number of adaptation targets, we show the 1 target (1T), 2 target (2T) 4 target (4T) and 8 target (8T) curves reported in Krakauer et al. (2000). In this study, participants moved a stylus across a digitized tablet and adapted to a $30^{\circ}$ rotation. manuscript. Here participants were exposed to a $45^{\circ}$ rotation while reaching to a single target. At the end of the experiment, participants were exposed to an aftereffect block where they reached 3 times to 16 different targets spaced in varying increments around the unit circle. In this aftereffect block feedback was removed and participants were told to move straight to the target without re-aiming. This aftereffect block was used to construct a generalization curve. In Fig. 4-Supplement 2B we show data only from 2 relevant locations on this curve. The "target" condition represents aftereffects probed at the 
training target. The "aim" condition shows the aftereffect measured at $22.5^{\circ}$ away from the primary target, which was the target closest to the mean reported explicit re-aiming strategy of $26.2^{\circ}$.

We also use the study's implicit generalization curve (their Fig. 3A) in our SPE generalization model analysis. This curve is reproduced in Fig. 4A. We extracted only one side: the one pointing along the vector which connected the aiming direction and the adaptation target. We also normalized the curve by dividing by the maximum implicit learning they measured along the aiming direction. These data were extensively used in our generalization analysis in Appendix 6. All relevant details are provided there. We selected this study because implicit and explicit learning were dissociated and because CW and CCW were counterbalanced across participants (alleviating potential position-based biases). Note that all data were extracted using the primary source's figures with MATLAB's GRABIT utility.

\section{Maresch et al. $(2021)^{41}$}

To evaluate the competition and independence models, we analyzed how implicit and explicit learning varied across individual participants in a study conducted by Maresch and colleagues ${ }^{41}$. In this analysis, we collapsed across participants in the $C R, I R-E$, and IR-EI groups ( $n=40$ total). Note that we did not include participants in the IR-I group, because implicit learning was only measured at one timepoint, unlike the 3 other groups. In this task, participants reached to 8 targets ( $45^{\circ}$ between each target) while holding a robotic manipulandum. Participants were exposed to a $60^{\circ}$ rotation. Implicit learning and explicit strategy were probed in various ways throughout the experiment. Here, we used the authors' exclusion-based implicit and explicit learning measures. In other words, implicit learning was measured by telling subjects to stop aiming. Explicit strategy was estimated as the voluntary decrease in reach angle that occurred when participants were told not to aim (the difference between total adaptation and implicit learning). To calculate total adaption, we averaged the reach angle over the 40 terminal rotation trials. We analyzed subject-to-subject pairwise relationships between implicit learning, explicit strategy, and total adaptation in Fig. 5-Supplements 1A, D, and G. To analyze each pairwise relationship, we used linear regressions.

Lastly, in Fig. 5-Supplement 3D we show data collected by Maresch et al. ${ }^{41}$, reported in Fig. $4 \mathrm{~b}$ of the original manuscript. This study calculated implicit learning directly with exclusion trials and indirectly with aim reports. In Fig. 5-Supplement 3D we show data from the IR-E group. This group was comparable to our data because aim was reported intermittently (4 times every 80 trials), meaning that on most trials, aiming targets would not cause adaptation (only the primary target). In addition, there were 8 adaptation targets, which will widen implicit generalization. The probe condition in Fig. 5Supplement 3D corresponds to the total implicit learning measured at the end of adaptation by telling participants to reach without re-aiming. The "report" condition corresponds to total implicit learning estimated at the end of adaptation by subtracting the reported aim direction from the measured reach angle.

Haith et al. (2015) ${ }^{13}$

To investigate savings, Haith et al. ${ }^{13}$ used a forced preparation time task. Briefly, participants $(n=14)$ performed reaching movements to two targets, T1 and T2, under a controlled preparation time scenario. To control movement preparation time, four audio tones were played (at $500 \mathrm{~ms}$ intervals) and participants were instructed to reach coincident with the 4th tone. On high preparation time trials (High 
PT), target T1 was shown during the entire tone sequence. On low preparation time trials (Low PT), T2 was initially shown, but was then switched to target T1 approximately $300 \mathrm{~ms}$ prior to the 4th tone. High PT trials were more probable (80\%) than Low PT trials (20\%).

After a baseline period (100 trials for each target), a $30^{\circ}$ visuomotor rotation was introduced for target T1 only. After 100 rotations trials (Exposure 1), the rotation was turned off for 20 trials. After a 24 hr break, participants then returned to the lab. On Day 2, participants performed 10 additional reaching movements without a rotation, followed by a second $30^{\circ}$ rotation (Target T1 only) of 100 trials (Exposure 2). The experiment then ended with a washout period of 100 trials for each target.

We quantified the amount of savings expressed upon re-exposure to the perturbation, on High PT and Low PT trials. We measured savings using two metrics. First, we measured the rate of learning during each exposure to the perturbation using an exponential fit. We fit a two-parameter exponential function to both Low PT and High PT trials during the first and second exposure (we constrained the third parameter to enforce that the exponential begin at each participant's measured baseline reach angle). We compared the exponential learning rate across high PT trials, low PT trials, and Exposures 1 and 2 with a two-way repeated-measures ANOVA (two within-subject factors: PT and exposure number), followed by one-way repeated-measures ANOVA to test simple main effects (Fig. 6B, right).

We also quantified savings in a manner similar to that reported by Haith and colleagues $^{13}$; we calculated the difference between the reach angles before and after the introduction of the perturbation, during each exposure (Fig. 6C, 1st and 2nd columns). For High PT trials, we then computed the mean reach difference over the 3 trials preceding, and 3 trials following perturbation onset. Given their reduced frequency, for Low PT trials, we focused solely on the trial before and trial after perturbation onset. We used the same statistical testing procedure (two-way rm-ANOVA with follow-up simple main effects) to test for savings in the pre-perturbation and post-perturbation differences (Fig. 6C, right).

Finally, we also used a state-space model of learning to measure properties of implicit and explicit learning during each exposure. We modeled implicit learning according to Eq. (3) and explicit learning according to Eq. (7). In our competition theory, we used target error as the error in both the implicit and explicit state-space equations. In our SPE model, we used target error as the explicit system's error, and SPE as the implicit system's error.

The total reach angle was set equal to the sum of implicit and explicit learning. Each system possessed a retention factor and error sensitivity. Here, we asked how implicit and explicit error sensitivity might have changed from Exposure 1 to Exposure 2, noting that savings is related to changes in error sensitivity ${ }^{14,17,19,26,51}$. Therefore, we assumed that the implicit and explicit retention factors were constant across perturbations but allowed a separate implicit and explicit error sensitivity during Exposures 1 and 2 . Therefore, our modeling approach included six free parameters. We fit this model to the measured behavior by minimizing the following cost function using fmincon in MATLAB R2018a:

$$
\theta_{\text {fit }}=\underset{\theta}{\operatorname{argmin}} \sum_{n=1}^{N}\left(y_{1}^{(n)}-\hat{y}_{1}^{(n)}\right)^{2}+\left(y_{2}^{(n)}-\hat{y}_{2}^{(n)}\right)^{2}
$$

Here $y_{1}$ and $y_{2}$ represent the reach angles during the $1^{\text {st }}$ and $2^{\text {nd }}$ rotation. These reach angles are composed of High PT and Low PT trials. On Low PT trials, the reach angle is equal to the implicit process. 
On High PT trials, the reach angle is equal to the sum of the implicit adaptive process and the explicit

1714

1715

1716

1717

1718

1719

1720

1721

1722

1723

1724

1725

1726

1727

1728

1729

1730

1731

1732

1733

1734

1735

1736

1737

1738

1739

1740

1741

1742

1743

1744

1745

1746

1747

1748

1749

1750

1751

1752

1753 adaptive process.

We fit this model to individual participant behavior, in the case where implicit learning was driven by target errors (Eq. (1)), and also in the alternate case where it was driven by SPEs (Eq. (2)). The implicit and explicit model simulations in Fig. 6D (columns 1 and 2) represent the competition theory (target error learning). For the SPE model, these states are not shown, but model parameters are reported in Fig. 6E.

We used a two-way repeated-measures ANOVA to test whether error sensitivity differed across implicit and explicit learning (within-subject factor) and across exposures (within-subject factor). We used follow-up one-way repeated measures ANOVA to test for differences across exposures (separately for implicit and explicit learning) for the SPE model, after detecting a statistically significant main effect.

Finally, we also fit the target-error (Eq. (1)) model to the mean behavior across all participants in Exposure 1 and Exposure 2. We obtained the parameter set: $a_{i}=0.9829, a_{e}=0.9278, b_{i, 1}=0.0629$, $b_{i, 2}=0.089, b_{e, 1}=0.0632, b_{e, 2}=0.1078$. Note that the subscripts 1 and 2 denote error sensitivity during Exposure 1 and 2, respectively. These parameters were used for our simulations in Fig. 7 (see Competition Map).

\section{Experiment 4}

The competition theory (Fig. 7) predicted that more consistently suppressing explicit strategy, relative to the conditions used by Haith et al. $(2015)^{13}$ ), should reveal savings in the implicit system. That is, Haith et al. (2015) inhibited strategy only on $20 \%$ of all trials. Strategies were able to compete with the implicit system on the remaining $80 \%$ of trials. To test this prediction, we inhibited strategy on every trial in Exp. 4. To inhibit strategies, we limited reaction time using the procedure described above for Experiments 2 and 3. In Exp. 3, we observed that limiting movement preparation time drastically suppressed explicit reaiming (Fig. $3 \mathrm{~N}$ ). Limiting preparation time in Exp. 4 was effective in reducing reaction times (Fig. 8B, top row), even lower than the 300 ms threshold used by Haith and colleagues ${ }^{13}$.

Experiment 4 used the 4-target protocol reported in Visuomotor rotation. Apart from that, its trial structure was similar to that of Haith et al. ${ }^{13}$. After a familiarization period, subjects completed a baseline period of 10 epochs ( 1 epoch $=4$ trials for each target). At that point, we imposed a $30^{\circ}$ visuomotor rotation for 60 epochs (Exposure 1). At the end of this first exposure, participants completed a washout period with no perturbation that lasted for 70 epochs. At the end of the washout period, subjects were once again exposed to a $30^{\circ}$ visuomotor rotation for 60 epochs (Exposure 2).

We quantified savings in a manner consistent with Haith et al. ${ }^{13}$. First, we fit a two-parameter exponential function to the reach angle during Exposures 1 and 2 (third parameter was used to constrain the fit so exponential curve started at the reach angle measured prior to perturbation onset). Second, we also tested for differences in the initial response to the perturbation across each exposure. To do this, we calculated the difference between reach angle during Exposures 1 and 2 (Figs. 8A\&B, bottom row). We then calculated the difference in reach angle between the 5 epochs preceding and 5 epochs following rotation onset. Differences between these two savings indicators (rate and early learning) were tested with a mixed-ANOVA, to determine how adaptation differed across each perturbation exposure (within-subject) in Exp. 4 and Haith et al. (between-subject factor). Statistically 
significant interaction effects were followed by one-way repeated-measures ANOVA (testing simple

1755

1756

1757

1758

1759

1760

1761

1762

1763

1764

1765

1766

1767

1768

1769

1770

1771

1772

1773

1774

1775

1776

1777

1778

1779

1780

1781

1782

1783

1784

1785

1786

1787

1788

1789

1790

1791

1792

1793

1794

1795

main effect of exposure number). Results are shown in Fig. 8C.

\section{Experiment 5}

Lerner and Albert et al. ${ }^{19}$ demonstrated that anterograde interference slows the rate of learning after 5 min (also $1 \mathrm{hr}$ ), but dissipates over time and is nearly gone after $24 \mathrm{hr}$. Here we wondered if this reduction in learning rate could at least be in part driven by impairments in implicit learning. Because Lerner and Albert et al. ${ }^{19}$ did not constrain preparation time, one would expect that participants used both implicit and explicit learning processes. In Experiments 2-4, we isolated the implicit component of adaptation by limiting reaction time. We used the same technique to limit reaction time in Experiment 5. The experiment paradigm is described in Visuomotor rotation above. With that said, we used 8 adaptation targets as opposed to 4 targets, to match the protocol used by Lerner and Albert et al. ${ }^{19}$.

The perturbation schedule is shown in Figs. $9 A \& B$ at top. We recruited two groups of participants, a 5 min group ( $n=9)$, and a $24 \mathrm{hr}$ group $(n=11)$. After familiarization, all participants were exposed to a baseline period of null trials lasting 5 epochs ( 1 epoch $=8$ trials). Next participants were exposed to a $30^{\circ}$ visuomotor rotation for 80 cycles (Exposure A). At this point, the experiment ended. After a break, participants returned to the task. For the 5 min group, the second session occurred on the same day. For the $24 \mathrm{hr}$ group, participants returned the following day for the second session. At the start of the second session, participants were exposed to a $30^{\circ}$ visuomotor rotation (Exposure B) whose orientation was opposite to that of Exposure A. This rotation lasted for 80 epochs.

We analyzed the rate of learning by fitting a two-parameter exponential function to the learning curve during Exposures $A$ and $B$ (the third parameter was used to constrain the exponential curve to start from the behavior on the first epoch of the rotation). For each participant we computed an interference metric by dividing the exponential rate of learning during Exposure $B$, by that measured during Exposure A (Fig. 9C, blue). We tested how interference was impacted by passage of time between Exposures A and B ( 5 min or $24 \mathrm{hr}$ ) as well as by the preparation time condition (no limit in Lerner \& Albert et al., limit in Exp. 5) using a two-way ANOVA. In addition, we calculated each exponential's $x$-intercept (i.e., zero-crossing), which we used in the control analysis described below.

One potential issue with this technique, is that it does not consider differences in the initial errors experienced during re-exposure to the rotation (Figs. 9A\&B, bottom row), which could alter sensitivity to error ${ }^{26,40,78}$. To examine this, we recalculated learning rate during the second rotation exposure only after the zero-crossing in reach angle (i.e., the point at which the error reach $30^{\circ}$, as in the initial exposure). To estimate this zero-crossing point, we used the exponential model's $x$-intercept as described above. Then we used a two-way ANOVA (same as above) to test how this alternate interference metric was altered by time passage (between exposures) and preparation time.

Lerner and Albert et al. $(2020)^{19}$

Recently, Lerner and Albert et al. ${ }^{19}$ demonstrated that slowing of learning in anterograde interference paradigms is caused by reductions in sensitivity to error. Here, we re-analyze some of these data.

Lerner and Albert et al. ${ }^{19}$ studied how learning one visuomotor rotation altered adaptation to an opposing rotation when these exposures were separated by time periods ranging from $5 \mathrm{~min}$ to $24 \mathrm{hr}$. Here we focused solely on the 5 min group $(n=16)$ and the $24 \mathrm{hr}$ group $(n=18)$. A full methodological 
description of this experiment is provided in the earlier manuscript. Briefly, participants gripped a joystick with the thumb and index finger which controlled an on-screen cursor. Their arm was obscured from view using a screen. Targets were presented in 8 different positions equally spaced at $45^{\circ}$ intervals around a computer monitor. Each of these 8 targets was visited once (random order) in epochs of 8 trials. On each trial, participants were instructed to shoot the cursor through the target.

All experiment groups started with a null period of 11 epochs ( 1 epochs $=8$ trials). This was followed by a $30^{\circ}$ visuomotor rotation for 66 epochs (Exposure A). At this point, the experiment ended. After a break, participants returned to the task. For the 5 min group, the second session occurred on the same day. For the $24 \mathrm{hr}$ group, participants returned the following day for the second session. At the start of the second session, participants were immediately exposed to a $30^{\circ}$ visuomotor rotation (Exposure B) whose orientation was opposite to that of Exposure A. This rotation lasted for 66 epochs. Short set breaks were taken every 11 epochs during Exposures $A$ and $B$.

Here, as in the earlier work $^{19}$, we analyzed the rate of learning by fitting a two-parameter exponential function to the learning curve during Exposures $A$ and $B$ (the third parameter was used to constrain the exponential curve to start from the behavior on the first epoch of the rotation). For each participant we computed an interference metric by dividing the exponential rate of learning during Exposure B, by that measured during Exposure A (Fig. 9C, green). In addition, we also analyzed the reaction time of the participants during Exposure $B$. The mean reaction time over the first perturbation block is shown in Figs. 9A\&B (middle, green traces).

\section{Mazzoni and Krakauer (2006) ${ }^{2}$}

In this study, subjects sat in a chair with their arm supported on a tripod. An infrared marker was attached to a ring placed on the participant's index finger. The hand was held closed with surgical tape. Participants moved an on-screen cursor by rotating their hand around their wrist. These rotations were tracked with the infrared marker. On each trial, participants were instructed to make straight out-andback movements of a cursor through 1 of 8 targets, spaced evenly in $45^{\circ}$ intervals. A $2.2 \mathrm{~cm}$ marker translation was required to reach each target. Note that all 8 targets remained visible throughout the task.

Two groups of participants were tested with a $45^{\circ}$ visuomotor rotation. In the no-strategy group, participants adapted as per usual, without any instructions. After an initial null period, the rotation was turned on (Fig. 10A, blue, adaptation). After about 60 trials of adaptation, the rotation was turned off and participants performed another 60 washout trials (Fig. 10A, blue, washout). The break between the adaptation and washout periods in Fig. 10A, no-strategy, is simply for alignment purposes.

The strategy group followed a different protocol. After the null period, participants reached for 2 movements under the rotation (Fig. 10A, 2 cycles no instruction, red). At this point, the subjects were told that they made 2 errors, and that they could counter the error by reaching to the neighboring clockwise target (all targets always remained onscreen). After the instruction, participants immediately reduced their error to zero (point labeled instruction in red, Fig. 10A). They continued to aim to the neighboring target under the rotation throughout the adaptation period. Note that the directional errors became negative. This convention indicates overcompensation for the rotation, i.e., that participants are altering their hand angle by more than their strategic aim of $45^{\circ}$. Towards the end of the adaptation period, participants were told to stop re-aiming, and direct their movement back to the 
original target (Fig. 10A, do not aim, rotation on). Then after several movements, the rotation was turned off as participants continued to aim for the original target during the washout period.

In Fig. 10A we show the error between the primary target (target 1 ) and cursor during the entire experiment. In Fig. 10B we show the error between the aimed target (target 2) and cursor during the adaptation period. Note that the aimed and primary targets are generally related by $45^{\circ}$ when the strategy group is re-aiming. We observed that initial adaptation rates (over first 24 movements, gray area in Fig. 10B) were similar, but the no-strategy group ultimately achieved greater implicit adaptation. These data were all obtained by using the GRABIT routine in MATLAB 2018a to extract the mean (and standard error of the mean) performance in each group from the figures shown in the primary article.

We fit 1 of 3 models to the direction error during the adaptation period shown in Fig. 10B. In all cases we modeled explicit re-aiming in the strategy group as an aim sequence that started at zero during the initial two movements, and then $45^{\circ}$ for the rest of the adaptation period (i.e., after the instruction to re-aim). In the no-strategy group, we modeled explicit learning as an aim sequence that remained at zero throughout the adaptation period.

In Fig. 10D, we modeled implicit learning based on the state-space model in Eq. (3) and target error term defined in Eq. (1). This target error was defined as the difference between the primary target (i.e., the initial target displayed associated with task outcome) and the cursor. In Fig. 10E, we modeled implicit learning based on the state-space model in Eq. (3) and the aim-cursor error defined in Eq. (2). This aim-cursor error was defined as the difference between the aimed target (either $0^{\circ}$ or $45^{\circ}$ ) and the cursor. Fig. 10F, shows our third and final model. In this model, implicit learning in the strategy group was modeled using the dual-error system shown in Eq. (6). That is, there were two implicit modules, one as in Fig. 10E. The evolution of these errors is shown in Fig. 10G. In the no-strategy group, we modeled implicit learning based on the primary target error and cursor alone.

Each model in Figs. 10D-F was fit in an identical manner. We fit the implicit retention factor and implicit error sensitivity to minimize squared error according to:

$$
\theta_{f i t}=\underset{\theta}{\operatorname{argmin}} \sum_{n=1}^{N}\left(y_{\text {strategy }}^{(n)}-\hat{y}_{\text {strategy }}^{(n)}\right)^{2}+\left(y_{\text {no-strategy }}^{(n)}-\hat{y}_{\text {no-strategy }}^{(n)}\right)^{2}
$$

In other words, we minimized the sum of squared error between our model fit and the observed behavior across the strategy and no-strategy groups in Fig. 10B. In other words, we constrained each group to have the same implicit learning parameters. In the case of our dual-error model in Fig. 10F, we assumed that each implicit module also possessed the same retention and error sensitivity. In sum, all model fits had two free parameters (error sensitivity and retention) which were assumed to be identical independent of instruction. This fit was performed using fmincon in MATLAB R2018a. The predicted behavior is shown in Figs. 10D-F at bottom. For our best model (Fig. 10F), the model behavior is also overlaid in Fig. 10B.

\section{Taylor and Ivry (2011)}

1875

In Fig. 10I, we show data collected and originally reported by Taylor and Ivry ${ }^{8}$. In this experiment, participants moved their arm at least $10 \mathrm{~cm}$ towards 1 of 8 targets, that were pseudorandomly arranged

1877 in cycles of 8 trials. Only endpoint feedback of the cursor position was provided. The hand was slid along 
the surface of a table while the position of the index finger was tracked with a sensor. After an initial familiarization block (5 cycles), participants were trained how to explicitly rotate their reach angle clockwise by $45^{\circ}$. That is, on each trial they were shown veridical feedback of their hand position, but were told to reach to a neighboring target, that was $45^{\circ}$ away from the primary illuminated target. After this training and another null period, the adaptation period started where the cursor position was rotated by $45^{\circ}$ in the counterclockwise direction for 40 cycles. The first 2 movements in the rotation exhibited large errors (Fig. 10I, 2 movements no instruction). As in Mazzoni and Krakauer², the participants were then instructed that they could minimize their error by adopting the aiming strategy they learned at the start of the experiment. Using this strategy, participants immediately reduced their direction error to zero.

Here we report data from two critical groups in this experiment. In the "instruction with target" group (Fig. 10I, black, $\mathrm{n}=10$ ) participants were shown the neighboring targets during the adaptation period to assist their re-aiming. However, in the "instruction without target" group (Fig. 10l, yellow, $\mathrm{n}=10$ ) participants were only shown the primary target; the neighboring targets did not appear on the screen to help guide re-aiming. Only participants in the "instruction with target" group exhibited the drift reported by Mazzoni and Krakauer ${ }^{2}$. However, both groups exhibited an implicit aftereffect (Fig. 10 , aftereffect; first cycle of washout period as reported in Fig. $4 \mathrm{C}$ of the original manuscript ${ }^{8}$ ).

Data were extracted from the primary figures in Taylor and Ivry ${ }^{8}$ using Adobe Illustrator CS6. We used the means and standard deviations for our statistical tests on the implicit aftereffect in Fig. 10 I.

\section{Measuring properties of implicit learning}

Many of our model's predictions depended on estimates of implicit retention factor and error sensitivity. We obtained these using the Limit PT groups in Experiment 2 and 3. To calculate the retention factor for each participant, we focused on the no feedback period at the end of Experiment 2 (Fig. 3E, no feedback) and the no aiming period at the end of Experiment 3 (Fig. 3L, no aiming). During these error-free periods trial errors were hidden, thus causing decay of the learned behavior. The rate of this decay is governed by the implicit retention factor according to:

$$
y^{(n)}=a_{i}^{n} y_{s s}
$$

Here $y^{(n)}$ refers to the reach angle on feedback trial $n$, and $y_{s s}$ corresponds to the asymptotic behavior prior to the no feedback period. We used fmincon in MATLAB R2018a to identify the retention factor which minimized the difference between the decay predicted by Eq. (13) and that measured during the no feedback period. For Experiment 2, we obtained an epoch-by-epoch retention factor of $0.943 \pm 0.011$ (mean \pm SEM). Note that an epoch consisted of 4 trials, so this corresponded to a trial-by-trial retention factor of 0.985 . When modeling Neville and Cressman ${ }^{30}$ (Fig. 1), we cubed this trial-by-trial term because each cycle consisted of 3 different targets (final retention factor of 0.9565). For Experiment 3, we obtained an epoch-by-epoch retention factor of 0.899 (trial-by-trial: 0.9738).

Next, we measured implicit error sensitivity in the Limit PT group during rotation period trials. To measure implicit error sensitivity on each trial, we used its empirical definition:

$$
b^{\left(n_{1}\right)}=\frac{y^{\left(n_{2}\right)}-a^{n_{2}-n_{1}} y^{\left(n_{1}\right)}}{e^{\left(n_{1}\right)}}
$$


Eq. (14) determines the sensitivity to an error experienced on trial $n_{1}$ when the participant visited a

1918

1919

1920

1921

1922

1923

1924

1925

1926

1927

1928

1929

1930

1931

1932

1933

1934

1935

1936

1937

1938

1939

1940

1941

1942

1943

1944

1945

1946

1947

1948

1949

1950

1951

1952

1953

1954

1955

1956

1957 particular target $\mathrm{T}$. This error sensitivity is equal to the change in behavior between two consecutive visits to target $\mathrm{T}$, on trials $n_{1}$ and $n_{2}$ divided by the error that had been experienced on trial $n_{1}$. In the numerator, we account for decay in behavior by multiplying the behavior on trial $n_{1}$ by a decay factor that accounted for the number of intervening trials between trials $n_{1}$ and $n_{2}$. For each target, we used the retention factor estimated for that target with Eq. (13).

Using this procedure, we calculated implicit error sensitivity as a function of trial in Experiment 2. To remove any potential outliers, we identified error sensitivity estimates that deviated from the population median by over 3 median absolute deviations within windows of 10 epochs. As reported by Albert and colleagues ${ }^{26}$, implicit error sensitivity increased over trials. Eqs. (4) and (5) require the steady-state implicit error sensitivity observed during asymptotic performance. To estimate this value, we averaged our trial-by-trial error sensitivity measurements over the last 5 epochs of the perturbation. This yielded an implicit error sensitivity of $0.346 \pm 0.071$ (mean \pm SEM).

To corroborate this value, we compared our estimate to data reported in Kim et al., $(2018)^{40}$. Here, error sensitivity is reported as a function of error size across various experiments in Fig. $3 a$. These data are reproduced in Fig. 3-Supplement 1C. Note that error sensitivity increases as errors get smaller. For our analyses, we required steady-state error sensitivity, which is the error sensitivity reached at the end of the training period. Fig. 3-Supplement 1B shows how error in the PT-Limit group changed with adaptation. The terminal error (horizontal black line) corresponding to the steady-state condition was equal to about $7.6^{\circ}$ (Fig. 3-Supplement 1B). For this error, error sensitivity fell somewhere between 0.25 and 0.35 (see Fig. 3-Supplement 1C) according to Experiments 1 and 2 reported by Kim et al. (2018). Thus, our value 0.346 appeared in agreement with these data.

Finally, we conducted a similar analysis in Experiment 3. However, trial-by-trial behavior was more variable and overall adaptation was lower in this laptop-based experiment. Thus, to obtain a more stable steady-state implicit error sensitivity estimate, we averaged error sensitivity over the asymptotic period apparent in Fig. 3-Supplement 1D (cycles 37 to 60). The average error sensitivity was approximately 0.193 (Fig. 3-Supplement 1D). To corroborate this value, we calculated the terminal error in the Limit PT group. This value was approximately $13.1^{\circ}$ (Fig. 3-Supplement 1E). This error corresponded to an error sensitivity between about 0.13 and 0.22 (Fig. 3-Supplement 1F) according to Kim et al. (2018). Thus, our Limit PT error sensitivity estimate 0.193 was within this range.

\section{Appendix 1}

In the main text, the competition and independence models predict steady-state implicit learning, $x_{i}^{s s}$. But each theory applies to the entire timecourse, not solely asymptotic learning. Here, we will derive general expressions that apply on each trial $k$. We will then show that the scaling, saturation, and nonmonotonic phenotypes in Fig. 1, occur throughout the entire implicit learning timecourse (in the competition model).

\subsection{General model derivation}

Consider the state-space model where the implicit system adapts to target error driven by the rotation $r$, as in the competition model (this is the competition model simulated in Figs. 6 and 7): 


$$
x_{i}^{(n)}=a_{i} x_{i}^{(n-1)}+b_{i}\left(r-x_{i}^{(n-1)}-x_{e}^{(n-1)}\right)
$$

Recall that $a_{i}$ and $b_{i}$ are implicit retention and error sensitivity. The $x_{i}^{(\mathrm{n})}$ and $x_{e}^{(\mathrm{n})}$ terms represent implicit and explicit learning on a given trial (numerator). This equation can be rewritten recursively to represent $x_{i}^{(\mathrm{n})}$ with respect to all prior trials:

$$
x_{i}^{(n)}=\left(a_{i}-b_{i}\right)^{n-1} x_{i}^{(1)}+b_{i} \sum_{k=1}^{n-1}\left(a_{i}-b_{i}\right)^{n-k-1}\left(r-x_{e}^{(k)}\right)(\mathrm{A} 1.2)
$$

In the case where implicit learning starts at zero (naïve learner), this equation simplifies to:

$$
x_{i}^{(n)}=b_{i} \sum_{k=1}^{n-1}\left(a_{i}-b_{i}\right)^{n-k-1}\left(r-x_{e}^{(k)}\right)(\mathrm{A} 1.3)
$$

Equation A1.3 shows how the implicit system on trial $n$ is driven by the explicit system on all prior trials, the rotation, and the implicit error sensitivity and retention factor. An excellent approximation to this equation can be obtained by replacing $x_{e}{ }^{(k)}$ as the average $x_{e}$ across all prior trials (this approximation's accuracy can be seen in the red and cyan lines in Fig. 1-Supplement 3A; cyan shows true implicit learning in Eq. A1.3; red shows the approximation in A1.4 below. This approximation yields:

$$
x_{i}^{(n)} \approx b_{i} \frac{1-\left(a_{i}-b_{i}\right)^{n-1}}{1-\left(a_{i}-b_{i}\right)}\left(r-x_{e}^{a v g}\right)(\mathrm{A} 1.4)
$$

Eq. A1.4 is analogous to the competition model in Eq. (4) in the main text. It states that implicit learning on a given trial $n$ is approximately proportional to $\left(r-x_{e}^{a v g}\right)$, the difference between the rotation and the average explicit strategy used by the participant. Note that in the limit as $n$ goes to infinity (i.e., steadystate), we obtain the competition model in Eq. (4). The implication of Eq. A1.4 is that a linear competition between implicit learning and explicit learning can be observed throughout the adaptation process, not only in the asymptotic learning phase. Thus, the scaling, saturation, and non-monotonic phenotypes we describe in Fig. 1, can be observed throughout the adaptation process.

Finally, note that the derivations above apply for the independence (SPE) model in Eq. (5). For this model, simply set all $x_{e}$ terms in A1.1-A1.4 to zero.

\section{A1.2 Scaling, saturation, and non-monotonic phenotypes across the entire implicit learning timecourse} Here we illustrate the scaling, saturation, and non-monotonic implicit learning phenotypes produced by the competition model. In Fig. 1-Supplement 3A, we simulate the implicit and explicit response to a $30^{\circ}$ rotation using A1.1 and its explicit learning analogue. This means that the implicit and explicit systems are driven by target error. Note that the red line shows the implicit approximation in A1.4 above, the blue line shows exact implicit learning in A1.3 above, and the magenta line shows explicit strategy. In this simulation, we used the $a_{i}, b_{i}$, and $a_{e}$ parameters identified in our model fit to Haith et al., (2015), as in the competition map shown in Fig. 7.

The scaling, saturation, and nonmonotonic phenotypes in Fig. 1 are due to how the explicit system responds to changes in rotation size. When strategy increases slower than the rotation, implicit learning will increase in the scaling phenotype. When it increases at the same rate as the rotation, implicit learning will stay the same, leading to the saturation phenotype. When it increases more rapidly than the rotation, implicit learning will decreases as the rotation increases: the nonmonotonic phenotype. All these scenarios are depicted in Fig. 1-Supplement 1.

Thus, to produce these phenotypes, we must change the way explicit strategy 'responds' to error: least vigorously in the scaling phenotype, and most vigorously in the nonmonotonic phenotype. To do this we simulated implicit and explicit responses to $30^{\circ}$ and $45^{\circ}$ rotations and tuned explicit error 
sensitivity: $b_{e}$. For all $30^{\circ}$ simulations, $b_{e}$ remained 0.2 . To obtain the scaling implicit phenotype, explicit error sensitivity remained 0.2 in the $45^{\circ}$ simulation. To obtain the saturation implicit phenotype, we increased $b_{e}$ to 0.435 in the $45^{\circ}$ condition (i.e., the explicit system became more reactive to the higher rotation). Lastly, to obtain the nonmonotonic phenotype, we increased the explicit error sensitivity dramatically, to 0.93 . In Figs. 1-Supplement 3B\&C, we calculate implicit and explicit learning at various points across these simulations: from left to right, 5, 10, 20, 40, and 150 rotation cycles. The explicit responses are shown in inset $B$. Implicit responses are shown in inset $A$.

There are many critical things to note. Firstly, at all time points, changes in explicit error sensitivity produce 3 distinct levels: less explicit learning when $b_{e}=0.2$, medium explicit learning when $b_{e}=0.435$, and high explicit learning when $b_{e}=0.93$. These changes have dramatic effects on the implicit system. For the low explicit strategy level, the implicit system scales when the rotation increases from $30^{\circ}$ to $45^{\circ}$. For the medium explicit strategy level, the implicit system remains the same when the rotation increases from $30^{\circ}$ to $45^{\circ}$. For the high explicit strategy level, implicit learning decreases when the rotation increases from $30^{\circ}$ to $45^{\circ}$. Most critically, all 3 phenotypes occur at all phases in the implicit learning time course: as early as cycle 5, and as late as cycle 150 (compare each set of bars in Fig. 1Supplement 3B). The only thing that changes is the total difference (i.e., effect size) between $30^{\circ}$ and $45^{\circ}$ implicit learning, which is smallest and hardest to detect early in learning (e.g., cycle 5), and largest (easiest to detect) when implicit learning reaches its steady-state (e.g., cycle 150).

What this means is that the competition model does not solely pertain to asymptotic learning. The same phenomena that occur due to implicit-explicit competition, appear throughout all phases in the learning process. To simplify matters we have chosen in our main text to focus on steady-state learning, where the mathematical relationship between steady-state implicit and explicit learning converges and is easy to test (i.e., the competition equation).

\section{Appendix 2}

In Figs. $1 \mathrm{H}-\mathrm{L}$ we applied the competition and independence theories to the stepwise group in Exp. 1. While these models do not solely apply to asymptotic learning (see Appendix 1 ), here we analyze whether the exposures during B1 $\left(15^{\circ}\right), B 2\left(30^{\circ}\right), B 3\left(45^{\circ}\right)$, and B4 $\left(60^{\circ}\right)$, lasted long enough to achieve implicit steady-state adaptation. That is, the scaling phenotype we noted in the implicit response could be influenced by 2 factors: (1) rotation size, and (2) exposure time.

In the stepwise condition in Exp. 1 we observed that implicit adaptation increased across the $15^{\circ}, 30^{\circ}, 45^{\circ}$, and $60^{\circ}$ rotation blocks (Fig. 1-Supplement $4 A$; rm-ANOVA, $F(3,38)=99.9, p<0.001$, $\left.\eta_{p}{ }^{2}=0.735\right)$. Were these increase due to changing the rotation's magnitude, or additional accumulation in the implicit response over time which had not yet saturated? Comparison with other comparable data sets, suggests that implicit learning likely saturated during each block in the Exp. 1 stepwise group. Here, we will describe each data set in turn.

Most importantly, the abrupt rotation group provides a way to verify the timecourse of implicit learning in Exp. 1. Total implicit learning was assayed 4 times in the abrupt group. Each probe overlapped with a stepwise rotation period. For example, the initial probe in the abrupt condition occurred during B1, when implicit learning was measured in the $15^{\circ}$ stepwise rotation. Similarly, the B1, B2, and B3 implicit measurements in the abrupt group overlapped with the $30^{\circ}, 45^{\circ}$, and $60^{\circ}$ rotation periods is the stepwise groups. Implicit learning measures across all 4 abrupt periods are shown in Fig. 1- 
Supplement 4B. We tested whether total implicit learning varied across the 4 blocks in the abrupt condition. We did not detect a statistically significant effect of block number on implicit learning (rmlast blocks (paired t-test, $\mathrm{t}(35)=1.53, \mathrm{p}=0.134$ ). This indicated that in the $60^{\circ}$ rotation condition there was little to no change in implicit learning following B1. This suggests that a single rotation block was sufficient to achieve steady-state adaptation even in the largest rotation condition tested: $60^{\circ}$.

Does this generalize to smaller rotation sizes? While we did not measure implicit learning across

2046

2047

2048

2049

2050

2051

2052

2053

2054

2055

2056

2057

2058

2059

2060

2061

2062

2063

2064

2065

2066

2067

2068

2069

2070

2071

2072

2073

2074

2075

2076

2077

2078

2079

2080

2081 multiple blocks in the $15^{\circ}, 30^{\circ}$, and $45^{\circ}$ stepwise conditions, we used a very similar experimental protocol in Salomonczyk et al. $(2011)^{36}$ : here 3 learning targets were also used, separated by $45^{\circ}$ between targets, in an upper triangular wedge (the exact same conditions as Exp. 1). Note that these data provided another example where the implicit response scales with rotation size during a stepwise rotation sequence (Fig. 1-Supplement $4 \mathrm{C}$ ). That is, when we increased the rotation in a stepwise manner across 3 blocks: $30^{\circ}$ then $50^{\circ}$ and lastly $70^{\circ}$, we observed strong increases in asymptotic implicit learning $(p<0.001)$. In this study we exposed another participant group to a $30^{\circ}$ rotation and measured implicit learning across 3 consecutive blocks (Fig. 1-Supplement 4D). Critically, we did not detect any change in implicit learning across the 3 learning periods (all contrasts, $p>0.05$ ). This matched our $60^{\circ}$ rotation analysis in the abrupt group in Exp. 1 . Thus, for both $30^{\circ}$ and $60^{\circ}$ rotations, a single block provides enough time to reach steady-state learning.

Lastly, we did not measure extended exposures to a $15^{\circ}$ or $45^{\circ}$ rotation, but Neville and Cressman $(2018)^{30}$ tested prolonged exposure to a $20^{\circ}$ and $40^{\circ}$ rotation in a similar paradigm ( 3 targets, separated by $45^{\circ}$ in an upper triangular wedge). No-instruction group implicit learning is shown in Fig. 1Supplements $4 \mathrm{E}$ and $\mathrm{F}$. We do not have access to the raw data, and so cannot run a repeated-measures ANOVA to test whether these groups exhibited a block-by-block change in implicit learning. However, comparing the first and third rotation blocks, we can calculate a maximum $0.7-1.4^{\circ}$ change in implicit learning. This represents a change between $7.6-16.5 \%$, which are daunted by the $313 \%$ increase in implicit learning exhibited from the $15^{\circ}$ to the $60^{\circ}$ blocks in the Exp. 1 stepwise group.

In sum, the $60^{\circ}$ implicit learning timecourse we measured in the abrupt group, the $30^{\circ}$ implicit learning timecourse measures in Salomonczyk et al. (2011), and the $20^{\circ}$ and $40^{\circ}$ groups in Neville and Cressman (2018), suggest that the implicit learning system exhibits little to no increase beyond the initial learning block. Each study was extremely comparable, testing participants with 3 learning targets, located in an upper triangular wedge spanning $45-135^{\circ}$ in the workspace. We estimate that any changes in implicit learning due to exposure time alone were limited to about $1.4^{\circ}$, which is an order magnitude smaller than that observed across the stepwise blocks in Exp. $1\left(14.5^{\circ}\right)$. Therefore, our Exp. 1 analyses in Figs. 1, 2, and 4, likely reflects steady-state implicit learning, or very nearly so.

\section{Appendix 3.}

In Section 1.1, we note a potential concern in our Fig. 1 analysis, where our implicit and explicit learning measures were not independent; explicit strategy was estimated as total adaptation - implicit learning: $x_{e}^{s s}=x_{T}^{s s}-x_{i}^{s s}$. Thus, when total adaptation is constant, implicit and explicit learning will exhibit a negative correlation simply due to the dependencies in our implicit and explicit learning measures. At a glance, this negative correlation may appear similar to the negative correlation between implicit and explicit learning embedded within the competition equation: $x_{i}^{s s}=p_{i}\left(r-x_{e}^{s s}\right)$. Thus, it is reasonable to 
question whether the correspondence between our empirical data and the competition model in Fig. 1, is unfairly biased by the intrinsic implicit-explicit dependencies in our empirical measures.

In short, suppose that two conditions, $A$ and $B$, exhibit an increase in implicit learning. Using our explicit learning measures, we would expect a decrease in explicit strategy. This negative correspondence between implicit learning and explicit strategy may appear trivially predicted by the competition model. This, however, is not the case. For this trivial relationship to occur, total adaptation must be the same in $A$ and $B$. In Fig. 1, we calculate implicit and explicit learning measures across changes in rotation size, over which total adaptation varies in proportion to rotation magnitude. Thus, our empirical implicit and explicit learning measures will not necessarily match the competition model.

To explain this, let us consider a hypothetical scenario similar to the data recorded by Neville and Cressman. Suppose total adaptation and implicit learning are measured over three rotations sizes $\left(20^{\circ}, 40^{\circ}\right.$, and $\left.60^{\circ}\right)$ : total learning $\left(19^{\circ}, 36^{\circ}, 53^{\circ}\right)$, implicit learning $\left(10^{\circ}, 10^{\circ}, 10^{\circ}\right)$. As expected, total adaptation increases with rotation size. Implicit learning remained constant, as in the Neville and Cressman data set. Next, suppose we estimate explicit strategy, by subtracting total adaptation and implicit learning. This will yield the explicit strategy estimates: $\left(9^{\circ}, 26^{\circ}, 43^{\circ}\right)$. Thus, in this example, our implicit and explicit learning measures are dependent. How well does the competition model match these data?

To answer this question, we can calculate the implicit learning gain, $p_{i}$, for each rotation size. This can be calculated as $p_{i}=x_{i}^{s s} /\left(r-x_{e}^{s s}\right)$. For the example above, the implicit learning gain would be equal to the set: $(0.909,0.714,0.588)$. What this means, is that as the rotation increases, the implicit learning gain decreases by approximately $35.3 \%$. These variations in $p_{i}$ will not produce a good match between the data and model. To show this, we can estimate the optimal $p_{i}$ that would minimize the squared error between the measured implicit learning, and model-predicted implicit learning. In this example, the optimal $p_{i}$ value is 0.693 . Using this gain, we can now use the model to predict how much implicit learning should occur in each rotation size condition. This would yield: $\left(7.62^{\circ}, 9.70^{\circ}, 11.78^{\circ}\right)$ in predicted learning. We can see even though implicit learning remained constant in the 'real' data, the competition equation predicts a 54.5\% increase in implicit learning across the 3 rotation sizes.

What does this mean? This hypothetical example demonstrates that calculating explicit learning via a subtraction between total adaptation and implicit learning, will not automatically yield a good match between the empirical data and the competition model. Rather, the only way that the competition model will match data, is in the case where the conditions tested in an experiment abide by the principle that $p_{i}$ is constant (or at least very similar) across various experimental conditions (e.g., rotation sizes). The three data sets we analyze in Fig. 1, must obey this property, and thus, are intrinsically compatible with Eq. (4).

Nevertheless, there is another way to corroborate the relationship between the model and data in Fig. 1 that does not involve using implicit and explicit learning measures at the same time. We described this method in Section 1.1. By noting that $x_{e}^{s s}=x_{T}^{s s}-x_{i}^{s s}$, we can substitute this expression into Eq. (4) to obtain a relationship between implicit learning and total adaptation: $x_{i}^{s s}=p_{i}\left(1-p_{i}\right)^{-1}\left(r-x_{T}^{s s}\right)$. This equation represents an alternate way to express the competition model which can be compared with the empirical data. Fortunately, here we compare $x_{i}^{s s}$ and $x_{T}^{s s}$ which were directly measured on separate trials (they are not dependent, in a statistical sense). 
A simple thought experiment shows that when data do not agree with the competition model, $x_{i}^{s s}$ and $x_{e}^{s s}$ can show a correlation, but $x_{i}^{s s}$ and $x_{T}^{s s}$ will not (in general). Suppose 3 participants have $10^{\circ}$, $15^{\circ}$, and $5^{\circ}$ implicit learning, respectively, and all have $20^{\circ}$ total learning. Subtracting total learning and implicit learning will yield an estimated $10^{\circ}, 5^{\circ}$, and $15^{\circ}$ explicit strategy, respectively. The competition model will predict that implicit-explicit and implicit-total will show negative correlations. For these participants, the implicit-explicit correlation is -1 , but the implicit-total correlation is 0 , inconsistent with competition. That is, implicit-explicit show a correlation spuriously, but implicit-total were sampled independently, yielding a robust way to test the competition theory.

Thus, in the main text we repeated our competition model analysis in Fig. 1 using the alternate equation. We used total adaptation measured in Exp. 1, Neville and Cressman, and Tsay et al., $2021^{35}$ to predict implicit learning. To do this, we identified a $p_{i}$ value in each data set that minimized the squared error between measured implicit learning and model-predicted implicit learning (in Neville and Cressman, the $p_{i}$ value was calculated across 6 conditions ( 3 rotations, 2 instruction types), in Exp. 1, the $p_{i}$ value was calculated across 5 conditions (all 4 rotation sizes in the stepwise group, as well as the $60^{\circ}$ abrupt condition), in Tsay et al., the $p_{i}$ value was calculated across all 4 rotation sizes).

Our results are shown in Fig. 1-Supplement 2. The competition model predicted nearly identical implicit learning patterns when total adaptation was used as the independent variable ('model-2') and when explicit adaptation was used as the independent variable ('model-1'). This control analyses shows that the close correspondence between measured behavior and model-predicted behavior in Fig. 1, is not due to the way we empirically operationalized our learning measures. Rather, the properties embedded in the competition model (with a constant implicit learning gain) were intrinsically present within the data.

\section{Appendix 4}

At various points in our work, we analyze how well the competition model, or independence model, can predict changes in implicit learning across various conditions. These models have one free parameter, the implicit learning gain: $p_{i}=b_{i}\left(1-a_{i}+b_{i}\right)^{-1}$. In each case, we assumed that the implicit learning gain is the same across experimental groups, i.e., only 1 gain is selected and applied to all experimental conditions. We made this choice to minimize the number of free variables. That is, allowing $p_{i}$ to vary across groups, would allow arbitrarily precise matches to the data. However, holding $p_{i}$ constant across conditions, shows that the same equation applies across conditions, limiting overfitting and increasing model confidence.

This assumption, however, may seem inappropriate given that the implicit learning gain depends on error sensitivity, which varies with conditions such as error size ${ }^{26,40,78}$. However, it is important to note that the implicit learning gain responds weakly to changes in error sensitivity. This insensitivity is due to the appearance of error sensitivity $\left(b_{i}\right)$ in both the gain's numerator and denominator: $p_{i}=b_{i}\left(1-a_{i}+b_{i}\right)^{-1}$. For example, let us suppose that participants in Condition 1 have $b_{i}=0.2$, but in Condition 2 is only $b_{i}=0.3$, a $50 \%$ increase. For an implicit retention factor of 0.9565 (see in Methods: Measuring properties of implicit learning), the implicit learning gain in Condition 1 would be $p_{i}$ $=0.821$ versus $p_{i}=0.873$ in Condition 2 . Thus, even though implicit error sensitivity was $50 \%$ larger in Condition 2 , the implicit learning gain would change only $6.3 \%$. For a more extreme case where implicit 
error sensitivity was double (0.4) in Condition 2, there would still only be a $9.8 \%$ change difference in implicit learning gain.

For these reasons, there are no physiologic changes in $b_{i}$ that could create the $46.2 \%$ increase in implicit learning in Fig. $2 \mathrm{C}$ (ratio of no instruction to instruction groups), and $82.3 \%$ increase in learning in Fig. $2 \mathrm{G}$ (ratio of stepwise to abrupt). To show this, we conducted sensitivity analyses. Imagine that across two conditions, a reference condition, and a test condition, implicit sensitivity varies. We set the reference implicit error sensitivity to $0.1,0.1625,0.225,0.2875,0.35$. We chose these values, because steady-state implicit error sensitivity determines steady-state implicit learning, which varied between 0.1 and 0.35 in Exps. 2 and 3; see Fig. 3-Supplement 1). Then we calculated how much $p_{i}$ (total learning) will change, when this reference error sensitivity increases to a test error sensitivity: test error sensitivity was capped at the physiologic, yet exceedingly unlikely, upper bound of 1 . Because $p_{i}$ also depends on implicit retention, we also tested several possible values between 0.95 to 0.99 . The results are depicted in Fig. 2-Supplement 2. Each column denotes the same analysis, but with a different retention factor. Each line denotes a different reference error sensitivity. The $\mathrm{x}$-axis continuously varies the test error sensitivity.

For example, for the point highlighted by the black arrow in the second column: this point shows that total implicit learning will increase by about $20 \%$ (y-axis) in a scenario where implicit retention = 0.96, and implicit error sensitivity increases from 0.1625 (i.e., it is on the red line) in the reference condition to 0.8 (i.e., the $\mathrm{x}$-axis value) in the test condition. This example is illustrative. Here $b_{i}$ increase from 0.1625 to 0.8 , a $392 \%$ increase, but total implicit learning only increases $20 \%$. In sum, extreme variations in implicit error sensitivity, still produce small changes in total implicit learning.

In Fig. 2-Supplement 2, we indicated the $46.2 \%$ and $82.3 \%$ changes in Figs. $2 \mathrm{C}$ and $\mathrm{G}$ with dashed horizontal lines. Note how no curve crosses either level, even though these represent several hundred \% changes in implicit error sensitivity. Thus, it is not possible that variations in implicit error sensitivity could generate the changes in total implicit learning we examined in Fig. 2.

On the other hand, consider the competition model, $x_{i}^{s s}=p_{i}\left(r-x_{e}^{s s}\right)$. Distributing the $p_{i}$ term yields: $x_{i}^{s s}=p_{i} r-p_{i} x_{e}^{s s}$. Note that $p_{i}$ varied between about 0.6 to 0.8 in our studies, meaning that implicit learning is very sensitive to competition. The implicit system will decrease by approximately $60-80 \%$ each unit increase in explicit strategy. For these reasons, the competition model more readily produces large fluctuations in implicit learning, such as those observed in Fig. 2.

\section{Appendix 5.}

In Experiment 1 in the main text, we analyze how abrupt and gradual perturbation onset alters the steady-state distribution for implicit and explicit learning. In this appendix, we conduct a similar investigation for data collected by Saijo and Gomi $(2010)^{33}$. In the first section, we describe the results of our analysis. In the second section, we describe the experimental paradigm and detail the methods used in our analysis.

\subsection{Suppressing explicit strategy enhances procedural learning}

In the main text (Figs. 2D-G), we observed that gradual perturbations reduce explicit re-aiming relative to abrupt rotations. The competition theory predicts that these reductions in aiming will lead to increases in implicit adaptation. That is, suppose participants adapt with an explicit strategy (Fig. 2- 
Supplement 3B, aim, solid magenta line), but this strategy is then suppressed (Fig. 2-Supplement 3B, aim, dashed magenta line). Reductions in explicit strategy will increase the residual steady-state target error that drives implicit adaptation. Thus, Eq. (4) predicts that suppressing explicit aiming will increase implicit learning (Fig. 2-Supplement 3B, H2, right, compare dashed blue and solid black implicit lines). However, a model where the implicit system responds to SPEs (Eq. (5)) does not predict any change in implicit learning.

We corroborated these predictions in Experiment 1 (Figs. 2D-G). Here we describe similar data collected by Saijo and Gomi $(2010)^{33}$. Participants were exposed to an abrupt (Fig. 2-Supplement 3A, abrupt) or gradual (Fig. 2-Supplement $3 \mathrm{~A}$, gradual) rotation. The abrupt perturbation was immediately set to $60^{\circ}$, but the gradual perturbation reached this magnitude over time, in six $10^{\circ}$ steps. Each rotation size persisted for 36 trials ( 3 cycles, 12 adaptation targets). Participants in the abrupt condition adapted rapidly to the perturbation, greatly decreasing their target error to about $5^{\circ}$ over about 10 perturbation cycles (Fig. 2-Supplement 3C, abrupt). In the gradual group, target errors remained small throughout training. Curiously, total adaptation was smaller in the gradual condition; participants exhibited a terminal error nearly 3 times greater than the abrupt condition (Fig. 2-Supplement 3C, gradual).

At this point, the perturbation was abruptly removed, revealing large aftereffects in each group. Paradoxically, even though participants in the gradual group had adapted less completely to the rotation, they exhibited larger aftereffects (Fig. 2-Supplement 3F, data), which remained elevated throughout the entire washout period (Fig. 2-Supplement 3C, aftereffect).

Here we demonstrate that these patterns are consistent with the competition theory, provided we make several assumptions. First, we assume that the initial washout cycle is driven by implicit learning. While this is likely not entirely true, we should note that when Morehead et al. ${ }^{22}$ measured aim reports during washout with a $45^{\circ}$ rotation, participants appeared to immediately "turn off" their explicit strategy. Nevertheless, this assumption likely introduces some error in our modeling approach. Second, we assume that participants in the abrupt group used larger strategies than participants in the gradual group. This assumption seems reasonable, considering that we also measured larger strategies in the abrupt group in Experiment 1, as did Yin and Wei (2020) $)^{25}$. Furthermore, as shown in Fig. 2 in the primary manuscript ${ }^{33}$, subjects in the abrupt group exhibited a sharp increase in reaction time upon rotation onset, consistent with explicit strategy use ${ }^{32,44}$.

With these assumptions, we investigated how well the competition and independence models predicted the observed data. To simulate these models, we estimated the explicit strategies in each group. Neville and Cressman ${ }^{30}$ measured the explicit response to a $60^{\circ}$ rotation, demonstrating that participants re-aimed their hand approximately $35^{\circ}$ consistently over the adaptation period (see yellow points in Figs. 2-Supplement 3D\&E, explicit aim). This estimate agreed well with the data; participants in the abrupt condition adapted $55^{\circ}$ and exhibited an aftereffect of approximately $20^{\circ}$ (Fig. 2-Supplement $3 \mathrm{~F}$, data, abrupt), suggesting about $35^{\circ}$ of re-aiming. In the gradual group, we assumed that little to no re-aiming occurred. This also seemed consistent with the data; participants in the gradual group adapted approximately $40^{\circ}$ and exhibited an aftereffect of approximately $38^{\circ}$ (Fig. 2-Supplement 3F, data, gradual) suggesting $<5^{\circ}$ of re-aiming. Using these estimates, we constructed hypothetical explicit learning timecourses, as shown in Figs. 2-Supplement 3D\&E, explicit aim).

We next used the state-space model to simulate the implicit learning timecourse, in cases where the implicit system learned solely due to SPE (Fig. 2-Supplement 3D, implicit angle) or solely due to 
target error (Fig. 2-Supplement 3E, implicit angle), under the assumption that participants in both the abrupt and gradual groups had the same implicit error sensitivity $\left(b_{i}\right)$ and retention factor $\left(a_{i}\right)$. The parameter sets that yielded the closest match to the measured behavior (Fig. 2-Supplement 3C) are shown in Figs. 2-Supplement 3D\&E. In both cases, the models predicted that abrupt learning would be more complete than gradual learning (i.e., steady-state error is smaller in the abrupt condition).

However, the implicit states predicted by SPE learning and target error learning possessed a critical difference. According to Eq. (4), the target error model predicted that the total extent of implicit learning would be suppressed by explicit strategy in the abrupt condition, yielding a smaller aftereffect (Fig. 2-Supplement 2E, implicit angle). However, according to Eq. (5), the SPE model predicted that implicit learning should reach the same level, yielding identical aftereffects (Fig. 2-Supplement 3D, implicit angle).

In summary, the differences in aftereffects across the abrupt and gradual conditions (Fig. 2Supplement 3F, data) were accurately predicted by the competition theory (Fig. 2-Supplement 3F, competition), but not the independence equation (Fig. 2-Supplement 3F, indep.). Suppressing explicit strategy revealed competition between implicit and explicit systems which suggested that the implicit system predominantly responded to target error. Furthermore, it is interesting to note that these data were consistent with our observation that steady-state adaptation is greater when explicit learning is large and implicit adaptation is small (Figs. $5 G \& H$ ). These trends are consistent with competition (Figs. 5D-F).

\subsection{Methods used to analyze Saijo and Gomi (2010)}

2269

2270

To understand how suppressing explicit strategy might alter implicit learning, we considered data collected by Saijo and Gomi ${ }^{33}$. In one of their experiments, the authors tested how perturbation onset altered the adaptation process. Subjects were divided into either an abrupt $(n=9)$ or gradual group $(n=9)$, and reached to 1 of 12 targets, which were ordered pseudorandomly in each cycle of 12 trials. After a baseline period of 8 cycles, a visuomotor rotation was introduced. The perturbation period lasted 32 cycles. After this, the perturbation was removed for 6 cycles of a washout condition. Participants were exposed to either an abrupt rotation where the perturbation magnitude suddenly changed from $0^{\circ}$ to $60^{\circ}$, or a gradual condition where the perturbation magnitude increased over smaller increments $\left(10^{\circ}\right.$ increments that lasted 3 cycles each, Fig. 2-Supplement 3A).

Here, we considered why participants in the abrupt perturbation condition achieved greater adaptation during the rotation period (smaller error in Fig. 2-Supplement 3C) but exhibited a smaller aftereffect when the perturbation was removed. Our theory suggested that this may be due to competition. If the gradual condition suppressed explicit awareness of the rotation ${ }^{25}$, then Eq. (4) would predict increases in implicit learning which were observed in the aftereffects measured during the washout period (where explicit strategies were disengaged). However, the SPE model (Eq. (5)) would predict the same amount of implicit adaptation: the same aftereffect in each condition.

To test these hypotheses, we simulated implicit adaptation using the state-space model in Eq. (3). In Fig. 2-Supplement 3D, we used an SPE for the error term in Eq. (3). In Fig. 2-Supplement 3E, we used the target error for the error term in Eq. (3). We imagined that the total reach angle was determined based on the sum of implicit and explicit learning. However, these authors did not directly measure explicit strategies. Fortunately, Neville and Cressman ${ }^{30}$ measured explicit strategies using 
inclusion and exclusion trials during a $60^{\circ}$ abrupt rotation (yellow points, explicit aim in Figs. 2Supplement 3D\&E).

We used these measurements in our abrupt simulations. Neville and Cressman observed that explicit strategies rapidly reached $35.5^{\circ}$ and remained stable during adaptation. To approximate these data, we simulated abrupt explicit strategy using the exponential curve: $x_{e}=35.5-10 e^{-2 t}$ (Figs. 2Supplement 3D\&E, explicit aim, black line). Note that the nature of this exponential curve is entirely inconsequential to our analysis, apart from its saturation level. Outside of the rotation period, we assumed explicit strategy was zero. This is consistent with data from Morehead et al. ${ }^{22}$ that showed almost immediate disengagement in aiming strategy during washout (though this assumption likely introduced error into our modeling approach). For the gradual condition, we assumed explicit strategy was zero throughout the entire experiment (Figs. 2-Supplement 3D\&E, explicit aim, gradual), as the participants remained largely unaware of the rotation. This seemed consistent with the data; gradual participants adapted approximately $40^{\circ}$, and exhibited an aftereffect of about $38^{\circ}$, indicating a re-aiming angle less than even $5^{\circ}$. Note, our primary results (Fig. 2-Supplement $3 \mathrm{~F}$ ) were unchanged in a sensitivity test where we assumed $10^{\circ}$ of re-aiming in the gradual group (Fig. 2-Supplement 4).

Thus, our simulations included two free parameters: error sensitivity $\left(b_{i}\right)$ and retention faction $\left(a_{i}\right)$ for the implicit system. In each simulation, we assumed that these parameters were identical across the gradual and abrupt groups. To fit these parameters, we minimized the following cost function:

$$
\theta_{\text {fit }}=\underset{\theta}{\operatorname{argmin}} \sum_{n}\left(e_{\text {abrupt }}^{(n)}-\hat{e}_{\text {abrupt }}^{(n)}\right)^{2}+\sum\left(e_{\text {gradual }}^{(n)}-\hat{e}_{\text {gradual }}^{(n)}\right)^{2}
$$

2309

2310

2311

2312

2313

2314

2315

2316

2317

2318

2319

2320

2321

2322

2323

2324

2325

2326

2327

2328

2329

Eq. (10) is the sum of squared errors between the directional errors predicted by the model (Figs. 2Supplement 3D\&E, directional error) and observed in the data (Fig. 2-Supplement 3C) across all trials in the abrupt and gradual conditions. Note that each simulation incorporated variability. We simulated noisy directional errors using the standard errors shown in the data in Fig. 2-Supplement $3 \mathrm{C}$. In the explicit state, we added variability to each trial using the standard error in explicit strategy reported by Neville and Cressman $^{30}$. For the implicit state, we used $20 \%$ of the explicit variability, given that aiming strategies are more variable than implicit corrections ${ }^{12}$. We repeated these simulations 20,000 times, each time resampling our noise sources and then fitting our parameter set $\left(a_{i}\right.$ and $\left.b_{i}\right)$ by minimizing Eq. (10) with fmincon in MATLAB R2018a. The mean implicit curves for the SPE learning model and target error learning models are shown in Figs. 2-Supplement 3D\&E respectively (implicit angle; mean \pm SD). Critically, in each simulation we measured the aftereffect that occurred on the first cycle of the washout period (Figs. 2-Supplements 3D\&E, aftereffect). The mean and standard deviation in these aftereffects is reported in Fig. 2-Supplemement 3F).

Finally, note that we obtained the directional errors in Fig. 2-Supplement $3 \mathrm{C}$ directly from the primary figure in the original manuscript (using the GRABIT routine in MATLAB R2018a). Please also note in the actual experiment, on some trials (7.1\% of all trials), the perturbation was introduced midway during the reach to test feedback corrections at only 1 target location (the $0^{\circ}$ target). These trials were not relevant for our current analysis. Otherwise, the visuomotor rotation was applied during the entire movement. Also note that because the authors analyzed feedback responses, participants made $15 \mathrm{~cm}$ movements, with a 0.6 second movement duration at baseline. Here, we only wanted to consider the feedforward adaptive component. Fortunately, the authors reported initial movement errors $100 \mathrm{~ms}$ 
following movement onset that could not have been altered by feedback. Therefore, we used these early measures of adaptation in the current study.

\section{Appendix 6}

Adapted movement patterns exhibit generalization: a decay in adaptation measured when participants reach towards new areas in the workspace ${ }^{46-48}$. Recent studies have observed that this generalization is centered where participants aim their movement, as opposed to the visual target ${ }^{49,50}$. In Exps. 1-3, we measured implicit adaptation by instructing participants to aim directly to the target without an explicit strategy. Had implicit learning truly been "centered" at the aiming location and not the target, these no aiming trials may underapproximate total implicit learning. This discrepancy will increase with re-aiming. Thus, it may appear that individuals who aim more, possess less implicit learning. These generalization properties could contribute to the negative subject-to-subject relationships we observed in Fig. 3 . In other words, could an SPE model with plan-based generalization produce the implicit-explicit correlations that we observed in Fig. 3? Our main text explores this possibility in Fig. 4. Here, we expand on these analyses to provide additional intuition, derivations, and technical background.

\subsection{Comparing our data to past generalization curves}

We compared data in Exps. 2 and 3 (Figs. 4A-C), with generalization curves measured by Krakauer et al. $(2000)^{47}$, Day et al. $(2016)^{49}$, and McDougle et al. $(2017)^{50}$. This last study is most important because aiming was controlled during generalization measurements and CW and CCW rotations were counterbalanced. In Fig. 4A we normalized our data so that true implicit learning equals measured implicit learning ( $100 \%$ generalization) when the re-aiming angle is $0^{\circ}$. To estimate total implicit learning (the value we normalized to), we used the $y$-intercepts corresponding to the regressions in Figs. $3 \mathrm{G}$ and 30 ( $25.5^{\circ}$ and $19.7^{\circ}$ in Exps. 2 \& 3) which extrapolates total implicit learning when explicit strategy is zero. Note that dimensioned data (in degrees) are shown in Figs. 4B\&C. As described in our Results, these comparison showed that implicit learning in Exps. 2 and 3 (Fig. 4A) declined nearly 300\% faster than the generalization curves predicted. For example, given the explicit strategies used in the No PT Limit groups in Exps. 2 and 3, McDougle et al. would predict about a $5^{\circ}$ reduction in implicit learning, whereas the actual data varied by about $15-20^{\circ}$. SPE generalization was too small in magnitude to match 2359 our data.

This analysis, however, has a critical limitation. Assuming that our implicit learning measures are altered by generalization, the explicit strategy we estimated will be affected too. That is, in Experiments 1-3 explicit strategy was estimated as total adaptation minus implicit learning. Had generalization reduced the implicit measures, it would falsely inflate our explicit measures. While it is tempting to compare our data in Figs. 2 or $4 \mathrm{~A}-\mathrm{C}$ with past generalization curves, this should not be done without correcting the explicit strategy measures. Such corrections create a substantial narrowing in the generalization curve. In Fig. $4 \mathrm{C}$ we show corrected implicit-explicit generalization curves that best match the data in Exps. 2 and 3. This process is described in A6.2. These curves had $\sigma=5.16^{\circ}$ and $5.76^{\circ}$ in Exps. $\left.37.76^{\circ}\right)$. Altogether, to obtain the implicit-explicit relationships we report in Exps. 2 and 3 would require unphysiological generalization curves that are an order of magnitude narrower than any study has reported to date. 


\subsection{Deriving SPE generalization models}

2374

2375

2376

2377

2378

2379

2380

2381

2382

2383

2384

2385

2386

2387

2388

2389

2390

2391

2392

2393

2394

2395

2396

2397

2398

2399

2400

2401

2402

2403

2404

2405

2406

2407

2408

2409

2410

2411

2412

2413

The competition model (Eq. (4)) can be represented as $x_{i}^{s s}=p_{i}\left(r-x_{e}^{s s}\right)$, where $p_{i}$ is an implicit learning gain (between 0 and 1). Multiplying out $p_{i}$ yields, $x_{i}^{s s}=p_{i} r-p_{i} x_{e}^{s s}$. Assuming $p_{i}$ is roughly constant (see Appendix 4 ), this expression predicts implicit learning with vary with explicit strategy according to a line with slope $-p_{i}$ and bias $p_{i} r$. In other terms, while the bias in implicit learning will increase with rotation size, the slope that relates implicit and explicit learning should not be altered by the rotation's magnitude.

Let us now derive an SPE generalization model. In this model implicit learning is driven by SPEs, and also exhibits plan-based generalization when it is measured. To derive this model, we begin with Eq. (5), the independence model: $x_{i}^{s s}=p_{i}$. This encodes SPE learning. Next, we add generalization; measured implicit learning (what we obtained when participants are instructed to stop aiming) is related to steady-state implicit learning by the generalization curve, $g$, which varies with steady-state explicit strategy, $x_{e}^{s s}$. Thus, we have: $x_{i}^{\text {measured }}=x_{i}^{s s} g\left(x_{e}^{s s}\right)$. Normally, this generalization curve would be modeling via a nonlinear cosine, or normal, tuning function. However, our data suggested that implicit and explicit varied linearly. Thus, we considered two model classes: (1) $g\left(x_{e}^{s s}\right)$ is linear and (2) $g\left(x_{e}^{s s}\right)$ is Gaussian. For the linear model, $g\left(x_{e}^{s s}\right)=1+m x_{e}^{s s}$ and for the normal model, $g\left(x_{e}^{s s}\right)=\exp \left(-\left(x_{e}^{s s} / \sigma\right)^{2}\right)$. By combining together all equations, we get: $x_{i}^{\text {measured }}=p_{i} r\left(1+m x_{e}^{s s}\right)$ for the linear model and $x_{i}^{\text {measured }}=p_{i} r \exp \left(-\left(x_{e}^{s s}\right)\right.$ $\sigma)^{2}$ ) for the normal model. Here $m$ is the generalization line's slope (negative), and $\sigma$ is the normal distribution's standard deviation.

For the linear model, this indicates that measured implicit learning and explicit strategy will vary according to a line with slope $p_{i} r m$ and bias $p_{i} r$. Thus, the rotation size will alter the slope relating implicit and explicit learning. In the Gaussian generalization model, nonlinearity also contributes to this variation. In the competition model, the slope does not vary with rotation size (see equation above). Thus a key way to compare competition with generalization is to examine whether the implicit-explicit slope changes with rotation size. This is discussed in more detail in A6.3.

Critically, $x_{e}^{s s}$ in the SPE generalization model is equal to the total steady-state explicit strategy. In Exps. 1-3, our Tsay et al., and our Neville and Cressman analysis, we did not calculate total explicit strategy. Rather our explicit strategies were estimated by subtracting total adaptation and implicit learning. Thus, we cannot fit the SPE generalization model to our data without correcting our explicit strategy measures as our explicit strategies will overapproximate true explicit strategy. To say this another way, suppose that the implicit learning we measured, $x_{i}^{\text {measured }}$ is less than total implicit learning $x_{i}^{s s}$. The explicit strategy we calculated was total adaptation, $x_{T}^{s s}$, minus $x_{i}^{\text {measured }}$. It should instead be $x_{T}^{s s}$ $-x_{i}^{s s}$. We need to correct the explicit measures in the SPE generalization model.

To do this, start with a Gaussian generalization model: (Eq. A6.1) $x_{i}^{\text {measured }}=x_{i}^{s s} \exp \left(-0.5\left(x_{e}^{s s} / \sigma\right)^{2}\right)$. Generalization causes a discrepancy between measured implicit learning and total implicit learning. The total amount that implicit learning is underapproximated, $x_{i}^{s s}-x_{i}^{\text {measured }}$, is equal and opposite to the total amount that explicit learning is overapproximated. Thus, we have (Eq. A6.2) $x_{e}^{\text {measured }}=x_{e}^{s s}+x_{i}^{s s}$ $x_{i}^{\text {measured }}$. Eq. A6.2 can be rearranged (Eq. A6.3): $x_{e}^{s s}=x_{e}^{\text {measured }}-x_{i}^{s s}+x_{i}^{\text {measured }}$. Combining Eqs. A6.1 and A6.3 yields the expression: (Eq. A6.4): $x_{e}^{s s}=x_{e}^{\text {measured }}-x_{i}^{s s}+x_{i}^{s s} \exp \left(-0.5\left(x_{e}^{s s} / \sigma\right)^{2}\right)$.

Equations $A 6.1$ and A6.4 together express and constrain the relationship between (1) total implicit learning, (2) total explicit learning, (3) measured implicit learning and (4) measured explicit 
learning. The same process can be used to correct the linear SPE generalization model. Here we begin with the implicit generalization line: (Eq. A6.5): $x_{i}^{\text {measured }}=x_{i}^{s s}\left(1+m x_{e}^{s s}\right)$. As above, the discrepancy $x_{i}^{s s}$ $x_{i}^{\text {measured }}$ will be equal and opposite to the discrepancy between $x_{e}^{s s}$ and $x_{e}^{\text {measured }}$. Thus, (Eq. A6.6) $x_{e}^{\text {measured }}=x_{e}^{s s}+x_{i}^{s s}-x_{i}^{\text {measured }}$.

Combining these equations together yields (Eq. A6.7) $x_{i}^{\text {measured }}=x_{i}^{s s}\left(1+m x_{e}^{\text {measured }} /\left(1-m x_{i}^{s s}\right)\right)$. We fit the SPE generalization models to the data noting that $x_{i}^{s s}=p_{i} r$ in the generalization model. These models have at 2 unknown parameters: $p_{i}$ and $m$ in the linear model, and $p_{i}$ and $\sigma$ in the normal model. In some cases (e.g., Figs. 4D-F), $m$ and $\sigma$ were set equal to the implicit generalization properties in McDougle et al. (more on this below). When these parameters were set, we used fmincon to identify the optimal $p_{i}$ that minimized the squared error between measured implicit learning, and the implicit learning predicted by Eqs. A6.1 (Gaussian) and A6.5 (linear) above. Note in the Gaussian model, we also needed to constrain that the model's solutions satisfy Eq. A6.4.

In other cases, we did not constrain $m$ or $\sigma$. Instead, we used fmincon (MATLAB R2021a) to identify the optimal $p_{i}$ and $m$ (linear), or $p_{i}$ and $\sigma$ (Gaussian), that minimized the squared error as described above.

\subsection{Comparing the competition model to SPE generalization models}

In Figs. 4D-F we compare the competition model to the SPE generalization models above. We did this in multiple ways. Note that the competition model possesses one unknown parameter, $p_{i}$. The SPE models possess two, $m$ and $p_{i}$ in the linear model, and $m$ and $\sigma$ in the normal model. In one analysis, we used the data collected by McDougle et al. (2017), to estimate the generalization parameters $m$ and $\sigma$. To estimate $m$, we used the initial two points on the generalization curve in Fig. 4A. This yielded $m=-0.011$. For the $\sigma$ parameter, we used the value calculated by McDougle et al. for their data: $\sigma=37.76^{\circ}$. These parameters were used in Figs. 4D-F, in the SPE gen. linear and SPE gen. normal models.

In Figs. 4D-F, we fit all 3 models to the stepwise group's implicit and explicit learning measures in B4 in Exp. 1 (the $60^{\circ}$ rotation period). The fitting procedure is described in A6.2 above. This revealed the optimal $p_{i}$ that best matched the implicit learning measures. Next, we used these parameters to predict the implicit-explicit relationship across the held-out rotation sizes (i.e., the B1, B2, and B3 periods). Each model's curve is shown in Fig. 4D.

To determine how well each model generalized to the held-out $15^{\circ}, 30^{\circ}$, and $45^{\circ}$ rotations, we calculated the RMSE between the model and measured data. We compared this error using an rmANOVA as reported in our Results (Section 1.6). Interestingly, the Gaussian model had slightly worse predictive power than the linear model (Fig. $4 \mathrm{E}, \mathrm{p}<0.01$ ). This was likely because the underlying data did not appear to be normally distributed.

Issues with the linear and normal SPE generalization functions were due to an intrinsic property possessed by the SPE generalization model; the relationship between implicit and explicit learning should vary as implicit learning increases. To understand this, suppose two participants have an explicit strategy of $20^{\circ}$, which hypothetically yields $80 \%$ generalization. If the first participant has $15^{\circ}$ implicit learning, they will exhibit a $15^{\circ}(0.2)=3^{\circ}$ decrease in implicit learning. If the second participant has $7.5^{\circ}$ implicit learning, they will exhibit only a $1.5^{\circ}$ decrease. What this means is that as total implicit learning changes, the gain that relates implicit learning and explicit learning will also vary. Because implicit learning increased with rotation size in Exp. 1 (stepwise), then the generalization curve will differ in 
slope across each period. For the Gaussian generalization model, there is an additional factor which alters the gain: sampling across the nonlinear distribution. As the rotation gets larger, explicit strategies increase, which results in systematic changes in where the normal distribution is sampled, hence yielding variable implicit-explicit relationships when assessed via a linear regression within the B1, B2, B3, and B4 periods.

These variations in slope/gain did not match the measured data (Fig. 4F). Here, we fit a separate well as the $95 \% \mathrm{Cl}$. Remarkably, the measured implicit-explicit slope appeared to be constant across all rotation sizes. This invariance was consistent with the competition theory (Fig. 4F, competition) which possesses an implicit gain $p_{i}$ that remains constant across rotations (like the data). It was not inconsistent with each generalization model, where slope varied across rotation sizes. Note, the error bars on model predictions in Fig. 4F were estimated with bootstrapping; we sampled participants with replacement, fit the models to collapsed participant behavior, and calculated their slope. For the Gaussian models, there is no one slope (nonlinear) so we calculated the slope in the region bounded by the explicit strategies seen in the measured data over each rotation period. Also note that any negative explicit strategies (only 3 points in the $15^{\circ}$ period and 1 in the $45^{\circ}$ period) were ignored during this calculation.

We also compared the models using AIC. For this analysis, we used the stepwise participants in Exp. 1. This is the only experiment where the model can be fit to individual participants, because implicit and explicit learning was assayed across the 4 rotation sizes. Thus, we fit all the models in Figs. 4D-F to individual participants. We used the same process described above, where $m$ and $\sigma$ were set to McDougle et al. values in the SPE generalization models. The results are shown in Fig. 4G. As expected, AIC strongly favored the competition model. At left, we show the generalization models' AIC values relative to that of the competition model (positive values mean competition is more likely to explain the data). At right, we show how many participants are best described by each of the 3 models.

A potential issue is that generalization properties differed between McDougle et al. and our data. Perhaps the SPE generalization would exhibited better performance with another $m$ or $\sigma$ value. To assess this possibility, we conducted a sensitivity analysis. We repeated the entire analysis in Figs. 4D-G described above, but varied $m$ and $\sigma$ across a wide range. For the range's lower bound we reduced the McDougle et al. generalization parameters by $50 \%$. For the upper bound we doubled the values. The sensitivity analysis results are shown in Fig. $4 \mathrm{H}$. The left inset shows the prediction error at each generalization width, similar to Fig. 4E. The right inset counts the total participants best explained by each model, according to AIC as in Fig. 4G. Across the entire range, the competition model had smaller error and better explained the data.

In sum, data in Exps. 1-3 were poorly explained by an SPE model extended with generalization. While plan-based generalization may promote negative implicit-explicit correlations, its contribution is small relative to the competition theory.

\subsection{Abrupt and gradual rotations in Exp. 1}

In Results Section 1.3, we observed that perturbing individuals in a stepwise manner led to an increase in implicit learning and a reduction in explicit strategy. These observations qualitatively and quantitatively matched the competition model (Figs. 2D-G). But there is an alternate possibility. Suppose 
that the implicit system is driven by SPE as in the independence model but exhibits plan-based generalization. In Appendix 6.2 we derive this SPE generalization model. This model could predict a reduction in implicit learning via two steps: (1) both abrupt and stepwise groups have equal implicit learning, but the abrupt rotation leads to greater re-aiming. (2) More aiming in the abrupt rotation results in a decrease in the implicit learning measured at the target due to plan-based generalization. This hypothesis could be summarized with an SPE generalization model in which measured implicit learning at the target is equal to total implicit learning via: $x_{i}^{\text {measured }}=x_{i}^{s s} \exp \left(-0.5\left(x_{e}^{s s} / \sigma\right)^{2}\right)$. Could this model lead to the observed data?

Initially, let us assume that $\sigma=37.76^{\circ}$ as measured by McDougle et al. ${ }^{50}$ To estimate the change in implicit learning between abrupt and gradual implicit learning, we can calculate the reduction in implicit aftereffect expected given a normal distribution with $\sigma=37.76^{\circ}$, for the $39.5^{\circ}$ and $29.9^{\circ}$ explicit strategies measured in the abrupt and stepwise groups. Aiming straight to the target in the abrupt group would yield $57.86 \%$ remaining implicit aftereffect. In the stepwise group, it would yield $73.09 \%$ remaining aftereffect. Altogether, the model predicts that stepwise implicit learning should increase by $100(73.09 / 57.86-1)$, or $26.3 \%$, over the abrupt group. On the contrary, the abrupt and stepwise implicit aftereffects were $11.72^{\circ}$ and $21.36^{\circ}$, respectively. This is an $82.3 \%$ increase in implicit learning.

In sum, similar to our analysis of Exps. 2 and 3 in Fig. 5, while generalization will produce a negative implicit-explicit relationship, the implicit learning variations we observed were much larger in Exp. 1 than predicted by generalization alone. In this case, the $82.3 \%$ increase in implicit learning is more than 3-fold larger than the $26.3 \%$ increase predicted by the implicit generalization properties measured by McDougle et al. (2017). Suppose $\sigma=37.76^{\circ}$ does not accurately represent our data. To match the measured data, $\sigma$ would need to be smaller, to narrow the generalization curve. This is unlikely to be the case given that in Exp. 1 used 3 targets, whereas McDougle et al. used 1. Additional targets do not narrow the generalization curve, they would widen it (Krakauer et al., 2000). Still, let us proceed. Rather than assume that $\sigma=37.76^{\circ}$, we can fit a normal distribution to the measured data. In the abrupt group, implicit and explicit learning were $11.72^{\circ}$ and $39.5^{\circ}$, respectively. In the stepwise group, implicit and explicit learning were $21.36^{\circ}$ and $29.9^{\circ}$, respectively. Fitting a normal curve to these data, would yield the curve shown in Fig. 4-Supplement $1 \mathrm{~A}$. The optimal $\sigma$ is $23.6^{\circ}$ and total implicit learning would need to be $47.8^{\circ}$.

While implicit learning equal to $47.8^{\circ}$, or $90 \%$ the total adapted responses, appears high, there is a more important issue. These values create an unphysical scenario. For abrupt learning an $x_{i}^{s s}=47.8^{\circ}$ and $x_{e}^{s s}=39.5^{\circ}$ would indicate that total adaptation should equal $47.8^{\circ}+39.5^{\circ}=87.3^{\circ}$ (Fig. 4 Supplement 1B). This is larger than the rotation's size and thus, is unphysical. In the stepwise group as well, total predicted learning would be about $77.7^{\circ}$, larger than the rotation size.

There is a deep issue here, as described in Appendix 6.2. The problem is that as the generalization curve narrows (e.g., $\sigma=23.6^{\circ}$ vs. $37.76^{\circ}$ ), not only does implicit learning measured at the target drastically underapproximate total implicit learning at the aim location, but the explicit strategy we estimated via $x_{e}^{s s}=x_{T}^{s s}-x_{i}^{s s}$ will substantially overestimate true explicit strategy. As $\sigma$ decreases, the issue will grow and the analyses above become inappropriate, leading to unphysical systems. To understand this, suppose $x_{i}^{s s}$ is larger than $x_{i}^{\text {measured }}$. Explicit strategy in Exp. $1, x_{e}^{\text {measured }}=x_{T}^{s s}-x_{i}^{\text {measured }}$. When this measured strategy is taken as actual explicit strategy in the generalization curve, this is equivalent to saying that total learning will be equal to $x_{e}^{\text {measured }}$, plus total implicit learning, $x_{i}^{\text {ss }}$. Total 
learning would be strategy $\left(x_{T}^{s s}-x_{i}^{\text {measured }}\right)$ plus total implicit learning $x_{i}^{s s}$. This would be $x_{T}^{s s}-x_{i}^{\text {measured }}+x-$ $i_{i}^{s s}$. Herein lies the contradiction. Because $x_{i}^{s s}$ is larger than $x_{i}^{\text {measured }}$, by using the estimated explicit strategy as the actual explicit strategy in the model, total learning in the model will automatically be larger than actual total learning. As we described above, this problem can progress so far as to predict that total learning is larger than the rotation.

The key idea is that both implicit and explicit learning need to be corrected by the generalization curve in our data. This correction is outlined in Appendix 6.2. Using Eqs. A6.1 and A6.4, we identified the $\sigma$ and $x_{i}^{\text {ss }}$ that minimized the squared error between $x_{i}^{\text {measured }}$ predicted by an SPE generalization model, and the measured stepwise and abrupt implicit values. The model revealed that the optimal $\sigma$ and $x_{i}^{s s}$ were $3.87^{\circ}$ and $45.69^{\circ}$, which produced the curve shown in Fig. 4-Supplement $1 \mathrm{C}$ (corrected model). This curve shows how measured implicit learning and explicit learning will interact. This is not the true implicit- explicit generalization curve. That curve is in Fig. 4-Supplement 1D (corrected model). The generalization curve required by the model, was implausible: it had a width of $\sigma=3.87^{\circ}$, compared to $37.76^{\circ}$ as measured by McDougle et al. This is why the model's distribution in Fig. 4-Supplement 1D is so narrow.

The relationship between Figs. 4D-Supplements 1C\&D may not be intuitive. To explain how these curves are entwined, consider the stepwise learning point in inset C. This point lies roughly at $20^{\circ}$ implicit learning and $30^{\circ}$ explicit strategy. This explicit strategy is the estimated strategy calculated in Exp. 1: total adaptation - measured implicit learning. Note that total implicit learning is about $45^{\circ}$. Thus, measured implicit learning is about $45-20=25^{\circ}$ smaller than total implicit learning. This means that our estimated explicit strategy at $30^{\circ}$, is about $25^{\circ}$ too large. Thus, the actual strategy is much smaller: $30-25$ $=5^{\circ}$. These corrections reveal the mapping between insets $\mathrm{C}$ and $\mathrm{D}$. The point, $x_{i}=20^{\circ}$ and $x_{e}=30^{\circ}$, will approximately lie at $x_{i}=20 / 45 \times 100=44.4 \%$, and $x_{e}=5^{\circ}$.

In sum, we conclude that our abrupt vs. stepwise analysis in Figs. 2D-G does not match implicit

2564

2565

2566

2567

2568

2569

2570

2571

2572

2573

2574

2575

2576

2577

2578

2579

2580 generalization. While incorrect, we began with the assumption that the implicit learning measures in Exp. 1 represent generalized learning, but explicit strategies represent total re-aiming. But in this model, the change in implicit learning in the actual data, was three times larger than that predicted by generalization as measured in McDougle et al. Moreover, this analysis is flawed, in that only correcting implicit measures with generalization, but not explicit measures, produced a situation where total adaptation would have exceeded the rotation's magnitude. This is because explicit strategies are estimated using total adaptation minus implicit learning. When we corrected the SPE generalization model so that both the implicit and explicit learning we measured were corrected by a generalization curve, the model required that plan-based generalization resemble a Gaussian with $\sigma=3.87^{\circ}$, an unphysiological scenario. The generalization model is not a viable alternate to the competition theory.

\subsection{Instructions and variations in rotation size}

In Neville and Cressman $(2018)^{30}$, implicit learning did not vary across $20^{\circ}, 40^{\circ}$, and $60^{\circ}$ rotations. Saturated responses like this resemble implicit learning properties exhibited in invariant error-clamp ${ }^{1,40}$ paradigms. In such experiments, the implicit system appears to reach a ceiling that does not depend on the rotation's magnitude (at least when rotations are less than $90^{\circ}$ ). Does this same phenotype cause the saturation we explored using the competition model in Neville and Cressman (2018)? 
In isolation this might appear so, but there is one issue: the response to instruction. The authors also tested how implicit learning and explicit strategies responded to instructions. Coaching participants increased explicit strategy but decreased implicit learning. This variation in implicit learning would not be explained by invariant error-clamp implicit learning properties, which would predict that implicit learning should always saturate at the same level. One idea that could potentially rescue this alternate hypothesis is generalization; perhaps implicit learning truly is the same across the instruction group and no instruction group but only appears variable because instructed participants used larger strategies. This idea, however, would directly contradict the implicit response to rotation size. Supposing all groups had the same implicit learning, greater explicit strategy in the $40^{\circ}$ and $60^{\circ}$ rotations, should produce a reduction in the measured implicit learning due to plan-based generalization.

In sum, an SPE learning model with a ceiling on implicit learning, would require complete $(100 \%)$ generalization, to show the saturation phenotype in Fig. 1D. However, there would be no way to capture the reduction in implicit learning seen in the instruction group with complete implicit generalization - a contradiction. Conversely, the reduction in implicit learning in the instruction group would need implicit generalization. But variations in explicit strategy across the $20^{\circ}, 40^{\circ}$, and $60^{\circ}$ rotations would alter implicit learning, violating the saturated implicit learning phenotype in the data. Thus, there is no way that these data can be described by an upper ceiling on implicit learning as in invariant error-clamp studies $^{1,40}$

As discussed in our Results, this is not true in the competition model. The exact same competition model parameters (i.e., implicit learning gain $p_{i}$ ) parsimoniously explained implicit responses to rotation size in Fig. $1 \mathrm{G}$ and instruction in Fig. 2C.

There is one last possibility to consider. Perhaps plan-based generalization alone could cause the decrease in implicit learning due to instruction, and the saturation in implicit learning across rotation size. In an SPE generalization model, the instruction and no-instruction groups could reach the same implicit learning level but show differences in implicit learning measured at the target due to variations in explicit strategy. In addition, implicit learning should scale according to $p_{i}$ as the rotation increases. Perhaps true implicit learning does vary across the $20^{\circ}, 40^{\circ}$, and $60^{\circ}$ rotation periods, but appears saturated because as rotation size increases so do strategies, reducing the implicit learning measured at the target due to generalization. We evaluated both these possibilities.

Let us begin with the response to rotation size. In Fig. 4-Supplements $1 \mathrm{E}$ and F, we fit a Gaussian SPE generalization model to the implicit and explicit responses measured in the no-instruction group. As described in Appendix 6.2, inset $E$ shows uncorrected explicit strategy estimates: total adaptation minus implicit learning. Inset $F$ shows the true implicit-explicit plan-based generalization curve that produces the data in inset E. These curves were produced by an implicit learning gain $p_{i}=0.56$, and $\sigma=11.2^{\circ}$. This shows that a generalization curve could yield a saturation phenotype, as shown in inset $\mathrm{E}$. Here, the same implicit curve is shown $\left(p_{i}=0.56\right.$ and $\left.\sigma=11.2^{\circ}\right)$ but is scaled by the rotation size $r$ as predicted by the SPE model. Increases in implicit learning due to the rotation size are counterbalanced by increases in explicit strategy which generalize less at the target. However, while such a model produces a saturation phenotype, the generalization curve's width as shown in inset $F$, is not physiological. Rather, to produce the measured responses, the curve's width $\left(11.2^{\circ}\right)$ would need to be $70 \%$ narrower than the generalization properties measured by McDougle et al. $\left(\sigma=37.76^{\circ}\right)$. This extreme narrowing has not been observed in past studies. Moreover, the notion that generalization in Neville \& Cressman would be 
narrower than that in McDougle et al., is inconsistent with known implicit generalization properties ${ }^{47,49,50}$. As shown in the Krakauer et al. generalization curves in Fig. $4 \mathrm{~A}$, increasing the number of training targets in Neville \& Cressman (3 targets) would widen the generalization curve relative to McDougle et al., which only used 1 training target.

Next, we repeated the analyses described above, on the implicit-explicit responses to instruction

2628

2629

2630

2631

2632

2633

2634

2635

2636

2637

2638

2639

2640

2641

2642

2643

2644

2645

2646

2647

2648

2649

2650

2651

2652

2653

2654

2655

2656

2657

2658

2659

2660

2661

2662

2663

2664 in Fig. 4-Supplements $1 \mathrm{G} \& \mathrm{H}$. The best generalization model $\left(p_{i}=0.5\right.$ and $\left.\sigma=9.8^{\circ}\right)$ could produce changes in generalized implicit learning that were consistent with the data, as shown in inset $G$. However, as in the response to rotation size described above, the required generalization properties were not physiologically consistent with past measurements as shown in inset $\mathrm{H}$. The generalization curve would need to be about $74 \%$ narrower than that measured by McDougle et al. Thus, again, while in principle generalization could produce changes in implicit learning, it would require implausible implicit learning properties.

6.6 Nonmonotonic implicit learning in Tsay et al. $(2021)^{35}$

In Tsay et al. $(2021)^{35}$ participants exhibited a non-monotonic implicit response to $15^{\circ}, 30^{\circ}, 60^{\circ}$, and $90^{\circ}$ rotations as shown in Fig. $1 \mathrm{~N}$. In the main text, we explain how this phenotype could be explained by the competition model. Namely, variations in strategy, could lead to changes in the residual target error that drives implicit learning in the competition model. Could another model also produce these data?

We considered that the decrease in implicit learning observed in the $90^{\circ}$ rotation group resembles a pattern shown by the implicit process in invariant error-clamp paradigms ${ }^{1,40}$; rotations larger than $90^{\circ}$ cause a drop in implicit learning. Morehead et al. ${ }^{1}$ suggested that a similar drop occurs in response to standard rotations, at least when participants are told to ignore the cursor and aim to the target (i.e., they did not use explicit strategies). Thus, might it be that reductions in implicit learning in response to large rotations are an intrinsic property of the implicit system, rather than a phenomenon caused by error competition? The experiments conducted by Morehead et al., have another similarity to the Tsay et al. observations. In particular, in standard rotation conditions (plus a no aiming instruction), Morehead et al. observed that a $7.5^{\circ}$ rotation caused a reduction in implicit learning relative to larger rotation sizes tested. This decrease in implicit learning cannot be due to error competition, because participants did not aim in this study. In sum, could it be that both the increase in implicit learning between $15-30^{\circ}$ in Tsay et al. as well as the drop in implicit learning between $60-90^{\circ}$ are caused by the implicit system's intrinsic learning properties, versus a competition with explicit strategy?

This is unlikely. First, In Morehead et al., subjects in the $7.5^{\circ}$ standard rotation condition achieved complete adaptation: a total reach angle of $7.5^{\circ}$. This $7.5^{\circ}$ level was smaller than that achieved in a $7.5^{\circ}$ invariant error-clamp. To explain these results, Morehead et al., suggested that implicit learning stopped in the standard rotation condition, because the error was completely canceled (i.e., both the rotation and total implicit achieved were $7.5^{\circ}$, creating a $0^{\circ}$ error). In the error-clamp condition, error never decreased and continued to drive implicit learning to its saturation point.

The data in Tsay et al., however, cannot be explained by the error cancellation mechanism. In the

$15^{\circ}$ rotation in Tsay et al., implicit learning reached only $7.6^{\circ}$, and thus did not completely cancel the error. Morehead et al. would have predicted that implicit learning should continue until $15^{\circ}$ to cancel the error. Thus, unlike Morehead et al., the increase in implicit learning between the $15^{\circ}$ and $30^{\circ}$ 
rotations in Tsay et al. cannot be explained by an error cancellation mechanism. This increase clearly violates invariant error clamp learning properties, where implicit learning reaches the same saturation point across error sizes less than $95^{\circ}$, unless error is canceled. This same argument can be made in the scaling phenotype in Exp. 1, as well as the scaling phenotype observed earlier by Salomonczyk et al. ${ }^{36}$

Next, consider the decrement in implicit learning shown in Fig. $1 \mathrm{~N}$ with the $90^{\circ}$ rotation. It remains possible that this decrease is due to a rotation-insensitivity that is intrinsic to the implicit process (rather than error competition). However, it is error that drives learning, not rotations. While the large rotations used by Morehead et al. resemble the $90^{\circ}$ group in Tsay et al., target errors were totally mismatched in these two studies. In Morehead et al., participants in both the error-clamp and standard rotation groups were told not to aim and to ignore the cursor. Because there was no strategy, the implicit learning curve reached approximately $10^{\circ}$, leaving an $85^{\circ}$ target error. Past studies have shown that error sensitivity will be exceedingly small in response to such extreme errors ${ }^{40,78,79}$. In our view, this insensitivity to extremely large errors likely led to the attenuation in implicit learning observed in Morehead et al. Instructions to "ignore the cursor" may further exacerbate reductions in sensitivity to these large errors.

However, in Tsay et al., subjects were allowed to aim. Total learning reached about $85^{\circ}$, leaving a $5^{\circ}$ target error: an error much more inclined to drive implicit learning. Comparing steady-state adaptation to this $5^{\circ}$ residual error with the $85^{\circ}$ residual error in Morehead et al., is not reasonable in our view.

In sum, the increase in implicit learning in the $15^{\circ}$ and $30^{\circ}$ groups could only be described by error competition, not an error cancellation mechanism as in Morehead et al. Second, the residual target errors experienced in Morehead et al. were about $80^{\circ}$ larger in their $95^{\circ}$ rotation group, than the $90^{\circ}$ rotation group in Tsay et al. For these reasons, attenuation in implicit learning in these 2 studies was likely caused by differing mechanisms: a drastic reduction in target errors (the competition hypothesis) in Tsay et al., and an unresponsiveness to extreme target error in Morehead et al. (which could have been exacerbated by telling participants to ignore the cursor). For the learning patterns in Tsay et al., the competition model seems the most parsimonious choice, not only given its quantitative match to the data (Figs. $1 Q$ and Fig. 1-Supplement 2), but also because it alone (not the error-clamp learning properties in Morehead et al.) can explain implicit responses across the many other cases described in Figs. 1 and 2: abrupt and stepwise responses in Exp. 1 (as well as Salomonczyk et al.), rotation responses between $15-60^{\circ}$ in Tsay et al., as well as implicit behavior in Neville \& Cressman. This is not to mention that the implicit learning properties in Morehead et al. provide no clear way to interpret the pairwise relationships between implicit learning, explicit strategy, and total learning detailed at length in Figs. 3-5 at the individual-participant level.

\section{Appendix 7}

2702

2703

In Section 1.6, we consider how an SPE model might also predict negative correlations between implicit learning and explicit strategy. Suppose that implicit learning is driven by SPEs and is not altered by explicit strategy. However, a subject with a better implicit learning system (e.g., a higher implicit error sensitivity) will require less explicit re-aiming to reach a desired adaptation level. In other words,

2705 individuals with large SPE implicit learning may use less explicit strategy relative to those with less SPE implicit learning. Like the competition theory, this scenario would also yield a negative relationship 
between implicit and explicit learning, due to the way explicit strategies respond to variation in the implicit system. We will now show the diverging predictions this model makes, relative to the competition theory.

\subsection{Competition model predictions: implicit learning responds to variations in explicit strategy} Here we will start with the competition theory. In this model, the implicit system responds to variation in explicit strategy according to: $x_{i}^{s s}=p_{i}\left(r-x_{e}^{s s}\right)$. Clearly, this predicts a negative relationship between implicit learning and explicit strategy (Fig. 5D). Next, we note that total adaptation is given by $x_{T}^{s s}=x_{i}^{s s}+$ $x_{e}^{s s}$. We can solve for $x_{e}^{s s}$ and substitute this into the model, yielding the following relationship between implicit learning and total adaptation: $x_{i}^{s s}=p_{i}\left(1-p_{i}\right)^{-1}\left(r-x_{T}^{s s}\right)$. This is the expression tested in Appendix 3 where we analyzed our data in Fig. 1 using steady-state implicit learning and total adaptation. We can rearrange this equation, solving for $x_{T}^{s s}$ yielding the dual expression: $x_{T}^{s s}=r+p_{i}^{-1}\left(p_{i}-1\right) x_{i}^{s s}$. This expression is written within the inset in Fig. 5F. Both equation variants show that in a stable learning system $\left(p_{i}<1\right)$ that implicit learning and total adaptation will exhibit a negative relationship. We can repeat this analysis, but this time solve for $x_{i}^{s s}$, and substitute into Eq. 4 , to obtain a relation between explicit learning and total adaptation. This yields $x_{T}^{s s}=p_{i} r+\left(1-p_{i}\right) x_{e}^{s s}$. Note that this expression is provided in Fig. 5E. Again, noting that $p_{i}<1$, this predicts a positive relation between explicit strategy and total adaptation.

To summarize, the competition model makes three predictions about the pairwise relationships between implicit learning, explicit strategy, and total adaptation. First, as explicit strategies increase, this will tend to increase total adaptation (i.e., positive relation as in Fig. 5E). As explicit strategy increases, the residual target error will decrease, leading to less implicit learning. This predicts that implicit learning will exhibit a negative correlation with both explicit strategy (Fig. 5D) and total adaptation (Fig. 5F).

\subsection{SPE model predictions: explicit strategy responds to variations in implicit learning}

Now, let us suppose we have the opposite scenario to the competition model. In an SPE model, implicit learning does not respond to explicit strategy. Suppose implicit learning varies randomly across subjects (due to inter-subject variability in implicit learning properties, e.g., error sensitivity) and explicit strategy responds to this variability in implicit learning. In this framework, competition occurs but with a reversed causal structure. Now, assuming $x_{i}^{s s}$ is due to an independent SPE learning mechanism, this will yield a residual target error of $r-x_{i}^{s s}$. A negative relationship between implicit and explicit learning occurs in the event that explicit strategies respond in proportion to this residual target error: $x_{e}^{s s}=p_{e}(r-$ $\left.x_{i}^{s s}\right)$, where $p_{e}$ is an explicit learning gain. This equation is the same as the competition model in Eq. (4), where the roles of $x_{e}^{s s}$ and $x_{i}^{s s}$ are reversed. Thus, similar relationships between $x_{T}^{\text {ss }}$ and each system occur. Assuming that $p_{e}$ is less than 1 (i.e., the explicit system does not overcompensate for the remaining error, yielding total learning $>r$ ) then the relationship between total adaptation and implicit learning will now be positive, with $x_{T}^{s s}=p_{e} r+\left(1-p_{e}\right) x_{i}^{s s}$, and the relationship between total adaptation and explicit learning will now be negative: $x_{T}^{s s}=r+p_{e}^{-1}\left(p_{e}-1\right) x_{e}^{s s}$. Note that these expressions are provided in Figs. 5B\&C.

To summarize, the SPE model makes three predictions about the pairwise relationships between implicit learning, explicit strategy, and total adaptation. First, as implicit learning increases, this will tend 
to increase total adaptation (i.e., positive relation as in Fig. 5C). But as implicit learning increases, there is a smaller target error for the explicit system to correct, leading to less explicit strategy. This predicts that explicit strategy will exhibit a negative correlation with both implicit learning (Fig. 5A) and total adaptation (Fig. 5B). This provides a way to compare the competition and SPE models.

\subsection{Simulating variations in implicit and explicit learning across participants}

2755

We constructed Figs. 5A-F to provide more intuition on how to compare the competition and SPE model

2756 predictions described above. In these toy-simulations, we first fit $p_{i}$ and $p_{e}$ in the equations above to the implicit and explicit measures in the No PT Limit group in Exp. 3 yielding $p_{i}=0.669$ and $p_{e}=0.689$. These values are not important; the same qualitative behavior will occur provided they are between 0 and 1 . For SPE model simulations we used $p_{e}$. We assumed that implicit learning varied across participants according to a normal distribution. For the distribution's mean we calculated the average implicit learning measured in the No PT Limit group. For the distribution's standard deviation, we used $4^{\circ}$. Then, we calculated explicit learning according to $x_{e}^{s s}=p_{e}\left(r-x_{i}^{s s}\right)$ for each participant. We then simulated "measurements" of implicit and explicit learning by adding a normal random variable with mean zero and standard deviation $2^{\circ}$ to these simulated implicit and explicit learning measures. We simulated 250 participants in total.

Simulations for the competition theory were similar. Here, we simulated explicit strategies

\subsection{Comparing pairwise implicit-explicit-total correlations between competition and SPE models}

In this Appendix we show that both an SPE model and a target error learning model could exhibit negative participant-level correlations between implicit learning and explicit strategy. But their predictions diverge on the relationships between total adaptation and each individual learning system. Target error learning predicts a negative implicit-total correlation and positive explicit-total correlation. SPE learning predicts a positive implicit-total correlation and a negative explicit-total correlation. To test these predictions, we considered how total learning was related to implicit and explicit adaptation measured in the No PT Limit group in Exp. 3. Our observations closely agreed with the competition theory; greater explicit strategy was associated with greater total adaptation (Fig. 5G, $\rho=0.84, p<0.001$ ), whereas greater implicit learning was associated with lower total adaptation (Fig. $5 \mathrm{H}, \rho=-0.70, p<0.001$ ).

We repeated similar analyses across additional data sets that also measured implicit learning via exclusion (i.e., no aiming) trials: (1) the $60^{\circ}$ rotation groups (combined across gradual and abrupt 
groups) in Experiment $1,(2)$ the $60^{\circ}$ rotation groups reported by Maresch and colleagues ${ }^{41}$ (combined across the CR, IR-E, and IR-EI groups), and (3) the $60^{\circ}$ rotation group described by Tsay and colleagues ${ }^{35}$. We obtained the same result as in Experiment 3. Participants exhibited negative correlations between implicit learning and explicit strategy (Fig. 5-Supplements 1G-I), positive correlations between explicit strategy and total learning (Fig. 5-Supplements 1D-F), and negative correlations between implicit learning and total learning (Fig. 5-Supplements $1 \mathrm{~A}-\mathrm{C}$ ). These additional studies also matched the competition theory's predictions.

\subsection{Critical exceptions to these predictions}

2800

2801 The competition theory predicts on average that implicit learning will exhibit a negative correlation with total adaptation (across individual participants). However, this prediction assumes that implicit learning is only driven by target errors, a condition we explore more completely in Part 3. Second, it assumes that implicit learning properties $\left(a_{i}\right.$ and $b_{i}$, summarized with the gain $p_{i}$ above) are identical across participants, an unlikely possibility. Variation in the implicit learning gain (e.g., Participant $A$ has an implicit system that is more sensitive to error) will promote a positive correlation between implicit and total adaptation, that will weaken the negative correlations we described above. Two examples where this appears to occur are shown in Fig. 5-Supplement 2A. Inter-subject variability in the implicit learning gain can dominate inter-subject variability in explicit strategy, which would lead to a positive relationship between implicit learning and total adaptation. It should be noted that the converse is not true in the independence model. SPE learning rules will always promote a positive relationship between implicit learning and total adaptation and will not show a negative correlation, despite inter-subject variability in implicit and explicit learning gains. A more thorough discussion on these matters is provided in Appendix 8.

\section{Appendix 8.}

In Section 1.6, we detail how an SPE independence model, and a target error competition theory predict implicit and explicit learning should vary across participants. To review, the SPE model predicts (1) positive correlations between implicit learning and total adaptation, and (2) negative correlations between explicit strategy and total adaptation. On the other hand, the competition theory predicts (1) negative correlations between implicit learning and total adaptation, and (2) positive correlations between explicit strategy and total adaptation. We noted several datasets that supported the competition theory: our data in Experiment 3 (Figs. $5 \mathrm{G}$ and $\mathrm{H}$ ), experiments conducted by Maresch and colleagues $^{41}$ (Fig. 5-Supplements 1A, D, and G), our data in Experiment 1 (Fig. 5-Supplements 1B, E, and $\mathrm{H}$ ), and data collected by Tsay and colleagues ${ }^{35}$ (Fig. 5-Supplements 1C, F, and I). Here we detail a critical nuance in the competition theory's predictions that may result in little to no correlation between implicit learning and total adaptation.

\subsection{Subject-to-subject correlations in implicit learning within the competition theory}

2828 The competition theory (i.e., target error learning model) will not always produce a negative relationship between implicit learning and total adaptation. In Section 1.6, we explained that the competition theory, $x_{i}^{s s}=p_{i}\left(r-x_{e}^{s s}\right)$, does on average predict a negative correlation between implicit learning and 
implicit learning systems, but Participant $A$ has superior explicit strategy. Overall, this means Participant $A$ will adapt more to the perturbation. However, their greater strategy will create a smaller driving force for the implicit system, yielding less implicit learning. Thus, total adaptation is positively correlated with explicit strategy, but negatively correlated with implicit learning.

To restate this idea, in a target error model, between-subject variation in explicit strategy creates a negative relationship between implicit learning and total adaptation. However, these predictions rely on a key assumption; implicit learning properties must be the same across all participants to yield negative correlations. In other words, in our Participants $A$ and $B$ example, both participants were assumed to have the same $p_{i}$ parameter, a term that depends on implicit error sensitivity and retention. Between-subject variation in these implicit learning properties, however, will promote a positive relationship between total adaptation and implicit learning. Thus, it is entirely possible that the negative correlations promoted by between-subjects explicit variability can be negated by the positive correlations promoted by between-subjects implicit variability, yielding no correlation in some instances.

To illustrate this, consider the toy simulation in Fig. 5-Supplement 4A. At left, we simulate total implicit learning using the competition equation $\left(p_{i}=0.8\right)$ across 35 participants adapting to a $30^{\circ}$ rotation, whose explicit strategies vary according to a normal distribution (mean $=12^{\circ}$, S.D. $=4^{\circ}$ ). Note the strong negative relationship between implicit learning and total adaptation. At right, we show the same data (same explicit strategies) but introduce variability in implicit learning ( $p_{i}$ in the model is varied according to a normal distribution with mean $=0.8$, and S.D. $=0.1$ ). Even though these data arise from the same competition equation, adding between-subject variation in implicit learning properties yields zero correlation between implicit learning and total adaptation $\left(p=0.199, R^{2}=0.05\right)$.

The competition equation predicts that the correlation between implicit learning and total adaptation is uniquely susceptible to contamination with between-subject implicit variability. That is, while the correlation between implicit learning and total adaptation (Fig. 5-Supplement 4A, right) was not statistically significant, the same simulated data exhibited a strong positive correlation between explicit strategy and total adaptation (Fig. 5-Supplement $4 B$ right; $p<0.001, R^{2}=0.42$ ), and a strong negative correlation between implicit learning and explicit strategy (Fig. 5-Supplement 4C, right; $\left.p<0.001, R^{2}=0.77\right)$.

Thus, with implicit variability the competition theory can simultaneously exhibit no correlation between implicit learning and total adaptation, a strong positive correlation between explicit strategy and total adaptation, and a strong negative correlation between implicit learning and explicit strategy.

To conclude, correlative phenomena in the competition theory represent a balance between negative correlations induced by between-subject explicit variability, and positive correlations induced by between-subject implicit variability. Observing negative correlations is a probabilistic phenomenon. A given study can easily fail to yield a statistically significant correlation between total adaptation and implicit learning, yet still be governed by the competition equation. To maximize the probability of detecting a negative correlation between implicit learning and total adaptation, there are several critical factors that should be considered by the experimenter.

To describe these factors, we compare our data in Experiment 3 (Figs. $5 \mathrm{G}$ and $\mathrm{H}$ ) to experimental conditions where we detected no statistically significant correlation between implicit learning and total adaptation. These include the $30^{\circ}$ rotation groups collected in Tsay et al. $(2021)^{35}$ and 
Experiment 1 (Fig. 5-Supplement 2A, middle and right). These studies used similar experimental procedures, yet only our data in Exp. 3 yielded a statistically significant correlation between implicit learning and total adaptation. Here we describe 4 key factors that may have played a role in these differences. For each factor, we will perform simulations using the competition equation. Factors 1 and 2 deal with statistical power. Factors 3 and 4 deal with how changes in explicit strategy use alter the ability to measure correlations between implicit learning and total adaptation.

\subsection{Factor 1. Statistical power: total number of trials}

2882

Because correlations between implicit learning and total adaptation are a balance between two opposing variability sources, high statistical power will increase one's ability to detect them in an experiment. One simple way to increase this power, is to increase the total number of trials used to measure total adaptation and implicit learning. That is, each reaching movement is corrupted by motor variability. To better estimate total adaptation and implicit learning, averaging over more trials lessens the effect of trial-to-trial reach variability on subject-to-subject correlations. This can be especially problematic for the number of aftereffect trials used to measure implicit learning, which remain limited in many studies.

Consider the simulations in Fig. 5-Supplement 2B. These simulations show a power analysis where we vary the total number of aftereffect trials in simulation, to detect the probability that an experiment with 30 participants will yield a statistically significant correlation between implicit and total adaptation. Here, implicit learning is set by the competition equation. All simulation parameters are held still (e.g., explicit parameter variability, implicit parameter variability, mean explicit strategy; see Methods below) except the total number of aftereffect trials used to calculate implicit learning. That is, we average over simulated trials to calculate total learning, explicit strategy, and implicit learning. Each simulated trial differs due to motor execution noise (i.e., varied according to a normal distribution). We repeat each simulation 40000 times with 30 participants in each simulation, and calculate the total fraction of iterations where there was a statistically significant negative correlation between total adaptation and implicit learning (Fig. 5-Supplement 2B, red, left), no statistically significant correlation between total adaptation and implicit learning (Fig. 5-Supplement 2B, black, left), and a positive statistically significant correlation between these two variables (Fig. 5-Supplement 2B, green, left).

This power analysis qualitatively demonstrates that increasing the number of aftereffect trials greatly improves one's ability to detect a negative statistically significant correlation between total adaptation and implicit learning. We should note that our study (Experiment 3 ) is an outlier, in that we used a very large number of no feedback (and no aiming) trials to measure implicit learning: 80 trials. In cases where we did not detect a statistically significant correlation, the total aftereffect trial count was much smaller: Tsay et al. (2021) used only 20 trials to measure the implicit aftereffect and Exp. 1 used only 18 trials (Fig. 5-Supplement 2B, right). Thus, Exp. 3 was more likely to produce a negative correlation between implicit learning and total adaptation, given this experimental factor.

\subsection{Factor 2. Statistical power: motor variability}

The second factor that plays an important role in measuring subject-to-subject correlations, is also related to statistical power: motor variability. Like trial count (Factor 1 ), the more variable a participant's reaching movements are, the poorer one's estimate for total learning and implicit learning. To show 
this, we repeated our power analysis process described above (using the competition model), but this time held all simulation parameters constant, except trial-to-trial variability in executing a movement. We sampled this motor execution noise parameter for each participant; some simulated subjects had higher trial-to-trial variability than others. We gradually increased the mean motor noise parameter across participants, as well as the variation in motor noise across participants. Results are shown in Fig. 5-Supplement 2C.

Motor execution noise plays a strong role in detecting statistically significant negative correlations between implicit learning and total adaptation (Fig. 5-Supplement 2C, left, red); as motor execution noise increases, the probability of detecting a statistically significant correlation falls sharply. Therefore, studies where subjects have smaller trial-to-trial variability in reaching movements, will be more likely to detect negative correlations between total adaptation and implicit learning. For example, we calculated the trial-by-trial variability in reach angle during the no feedback periods in our data (Exp. 3 ) as well as the $30^{\circ}$ rotation datasets we described above (Tsay et al., 2021 and Experiment 1). We used this period so that trial-to-trial volatility in explicit strategy did not corrupt our estimate of motor variability (i.e., trial-to-trial variability is much larger during asymptotic behavior).

As shown in Fig. 5-Supplement $2 \mathrm{C}$ at right, participants in Experiment 3 exhibited smaller trialby-trial reach variability (one-way ANOVA, $F=6.84, p=0.002$ ) than both the Tsay dataset (post-hoc test: $p=0.003$ ) as well as Experiment 1 (post-hoc text: $p=0.015$ ). Thus, Exp. 3 was more likely to produce a negative correlation between implicit learning and total adaptation, given this experimental factor. In addition, it should be noted that motor noise variability (Factor 2) will act synergistically with limited aftereffect trials (Factor 1 ) to impair one's ability to detect accurate implicit learning measures.

While it may be difficult to control motor noise, experimenters should consider the following parameters: (1) movement displacement, (2) the type of experimental apparatus (laptop vs. robot vs. tablet), (3) the speed of the reaching or shooting movements, and (4) target location. These experimental conditions will alter reaching variability and may improve one's ability to detect negative correlations between total adaptation and implicit learning.

\subsection{Factor 3: explicit strategy use} Factors 3 and 4 relate less to statistical power, and more to the variability sources that underly subjectto-subject differences in implicit and explicit learning. A critical factor that determines one's probability of detecting negative correlations between implicit learning and total adaptation, is total explicit strategy use. To detect how overall strategy use affects the ability to obtain statistically significant correlations, we again used our power analyses. This time, we repeated our power analysis procedure, but varied the mean of the normal distribution used to simulate variable explicit strategies; we gradually increased the mean strategy use across our simulations, in the case where participants adapted to a $30^{\circ}$ rotation. All other simulation parameters remained constant across simulations $(n=30$ in each simulation, 40000 iterations for each explicit strategy level). The results are shown in Fig. 5-Supplement $2 \mathrm{E}$, at left.

Strategy use strongly affects one's ability to detect negative statistically significant correlations between implicit learning and total adaptation (Fig. 5-Supplement 2E, left, red). When participants use little explicit strategy on average, it is more difficult to obtain a statistically significant implicit-total adaptation correlation. In other words, at a given rotation size, studies where participants use greater 
strategies are more likely to yield a negative relationship between implicit learning and total adaptation. Comparing participants in Experiment 3 to the Tsay dataset and Experiment 1 (Fig. 5-Supplement 2E, right), we noted that participants in the Tsay dataset exhibited large reductions in explicit strategy use (one-way ANOVA, $\mathrm{F}=11.09, \mathrm{p}<0.001$; post-hoc tests had $\mathrm{p}<0.001$ for Experiment 3 vs. Tsay and Experiment 1 vs. Tsay). Thus, participants in the Tsay experiment were least likely to exhibit negative correlations between implicit learning and total adaptation, according to this experimental factor.

\subsection{Factor 4: Between-subject variability in explicit strategy use}

Recall that the relationship between implicit learning and total adaptation is a balance between variability sources: positive correlations induced by between-subjects implicit variability, and negative correlations induced by between-subjects explicit variability. Thus, more variability in explicit strategy increases how likely one is to detect a negative correlation between implicit and total adaptation. To demonstrate this, we performed a final power analysis. All parameters were constant across simulations, except variability in strategy use. For each simulation $(n=30)$ we sampled strategies from a normal distribution; we gradually increased the SD of this normal distribution across simulations (40000 simulations for each level) while holding mean explicit strategy constant. The results are shown in Fig. 5Supplement 2D, at left.

These simulations demonstrated two critical properties. First, as subject-to-subject variability in strategy use increases, so too does the likelihood of detecting a negative relationship between implicit learning and total adaptation (Fig. 5-Supplement 2D, left, red). Second, when between-subject explicit variability is very low, there is even a chance of detecting positive correlations between total adaptation and implicit learning (Fig. 5-Supplement 2D, left, green) even in the competition theory. This key point should be kept in mind when experiments use conditions where strategy use is minimal across participants (e.g., exceedingly gradual rotations; very small rotations, etc.).

Along these lines, we should note that between-subject variability in explicit strategy use was greatest in Experiment 3. As compared to the Tsay dataset and Experiment 1, explicit variability was 32\% and $72 \%$ greater in Experiment 3, respectively (Fig. 5-Supplement 2D, right). Therefore again, Experiment 3 was most likely to produce a negative correlation between implicit learning and total adaptation.

\subsection{Unique susceptibility in the correlation between implicit learning and total adaptation}

It is important to reiterate that with target error learning, negative correlations between implicit learning and total adaptation are uniquely challenging to detect. That is, there is more power to detect positive correlations between explicit strategy and total adaptation. For example, though we did not detect a negative relationship between implicit learning and total adaptation in the $30^{\circ}$ conditions tested by Tsay et al. (2021) and in Experiment 1, we did detect a positive correlation between explicit strategy and total adaptation in these experiments (Fig. 5-Supplements $3 \mathrm{~A}$ and $\mathrm{B}$ ).

Fig. 5-Supplement 3 (panels C-F), again shows the power analyses on Factors 1-4 illustrated in Fig. 5-Supplement 2, but this time investigates the correlation between explicit strategy and total adaptation. Across all 4 factors, the power analyses demonstrated that experiments should yield greater probability of detecting positive correlations between explicit strategy and total adaptation (Fig. 5Supplements $3 \mathrm{C}-\mathrm{F}$, green curves at top), than negative correlations between implicit learning and total 
adaptation (Fig. 5-Supplements 3C-F, red curves at top). These data are recapitulated in the simulated $\mathrm{R}^{2}$ statistic across the two correlations (Fig. 5-Supplement 3, panels C-F, bottom row); the correlation between total adaptation and explicit strategy was greater than the correlation between total adaptation and implicit learning.

\subsection{Appendix 8 summary}

Here we explained processes that impact the correlation between implicit learning and total adaptation in the competition theory. Between-subject variability in explicit strategy and implicit learning properties promote positive and negative correlations between implicit learning and total adaptation, respectively. This contention makes it possible that correlations between implicit learning may be weak or absent in an experiment. We explored 4 key experimental factors that researchers should consider in their data sets to maximize the chance of detecting negative correlations between implicit learning and total adaptation. However, this is by no means a complete list. For example, greater SPE learning will drastically undermine the negative correlations between implicit learning and total adaptation produced by target error learning. Thus, we expect that conditions which use multiple visual landmarks (e.g., aiming targets) are unlikely to show negative correlations between implicit learning and total adaptation.

\subsection{Appendix 8 methods}

Here we analyzed data collected in Experiment 1, Experiment 3, and Tsay et al. (2021). Implicit and explicit learning measures were calculated as reported in the Methods section in our main text. These implicit and explicit learning measures were used to calculate the correlations shown in Fig. 5Supplement $2 \mathrm{~A}$ and Fig. 5-Supplements $3 \mathrm{~A}$ and $\mathrm{B}$. In addition, the explicit measures were used to calculate the strategy use in Fig. 5-Supplement 2E. Each dot in the right-most inset represents an individual participant. Variations in explicit strategy across experiments were assessed with a one-way ANOVA, with Bonferroni-corrected post-hoc tests. In addition, Fig. 5-Supplement 2D (at right) shows the std. dev. in explicit strategy across participants within the 3 experimental conditions. In Fig. 5Supplement 2C, we estimated motor variability within individual participants. To do this we calculated the standard deviation in the reach angle across trials in the no-aiming period at the end of each experiment. We chose this period to prevent volatility in explicit strategy from inflating our motor variability measure. Each dot in the right-most inset shows the reach angle standard deviation for a single participant. We assessed differences in motor variability across the 3 experiments using a oneway ANOVA, with Bonferroni-corrected post-hoc tests.

In Fig. 5-Supplement 4, we provide toy simulations to illustrate how variation in implicit learning properties alters pairwise relationships between implicit learning, explicit strategy, and total adaptation. For the left-most inset in panels $A, B$, and $C$, we simulated a condition with no variability in implicit learning properties. That is, we used the competition equation to simulate implicit learning, but held $a_{i}$ and $b_{i}$ constant across all participants (each individual dot in the panel). We chose $a_{i}$ and $b_{i}$ so that the implicit learning gain, $p_{i}$, was equal to 0.8 . We simulated 35 participants adapting to a $30^{\circ}$ rotation. Explicit strategy was sampled for each participant using a normal distribution with a mean of $12^{\circ}$ and a standard deviation of $4^{\circ}$. The right-most inset in panels $A, B$, and $C$, use the exact same explicit strategies. However, here we allow $p_{i}$ (i.e., implicit learning properties) to vary across participants. To 
simulate this variation, we sample $p_{i}$ according to a normal distribution with a mean of 0.8 and a standard deviation of 0.1 .

Finally, Fig. 5-Supplement 2 and Fig. 5-Supplement 3 show 4 power analyses. The power analyses were the same across these two figures, only, Fig. 5-Supplement 2 focuses on how implicit learning relates to total adaptation, and Fig. 5-Supplement 3 considers how explicit strategy relates to total adaptation. In these power analyses, there are several parameters. First, implicit error sensitivity was uniformly sampled between 0.9 and 0.95 . Implicit error sensitivity was uniformly sampled between 0.2 and 0.3 . The rotation size was always $30^{\circ}$. Other simulation parameters varied across each power analysis. For each power analysis, there was one parameter that varied across simulations, but all other parameters were fixed to default values. The default values were as follows. Explicit learning was sampled for each participant using a normal distribution with a mean of $10^{\circ}$ and a standard deviation of $6^{\circ}$. The total number of trials used to measure total adaptation, implicit learning, and explicit learning was equal to 40 . Motor variability had a mean of $12^{\circ}$ across participants, with a standard deviation of $6^{\circ}$.

Power analyses in Fig. 5-Supplement 2B and Fig. 5-Supplement $3 \mathrm{C}$ used the default parameter values but varied the total number of probe trials used to measure implicit and explicit learning between 1 and 80. Power analyses in Fig. 5-Supplement 2C and Fig. 5-Supplement 3D used the default parameter values but varied the average motor variability between $5^{\circ}$ and $20^{\circ}$, and the standard deviation in motor variability between $2^{\circ}$ and $10^{\circ}$. As mean motor variability increased across simulations, so did the subject-level standard deviation. Power analyses in Fig. 5-Supplement 2D and Fig. 5-Supplement 3E used the default parameter values but varied the standard deviation in strategy use between participants between $0.1^{\circ}$ and $8^{\circ}$. Finally, power analyses in Fig. 5-Supplement $2 \mathrm{E}$ and Fig. 5-Supplement 3F used the default parameters values but varied average strategy use between $0^{\circ}$ and $20^{\circ}$.

In these power analyses, the parameter of interest was varied linearly between its two extreme values. For each value we conducted 40,000 simulations, each time sampling random variables for 30 participants according to the distributions noted above. Across these simulations we calculated the probability that a negative statistically significant relationship occurred between implicit learning and total adaptation (red lines in Fig. 5-Supplement 2 and Fig. 5-Supplement 3), a positive statistically significant relationship occurred between implicit learning and total adaptation (green lines in Fig. 5Supplement 2), no statistically significant relationship occurred between implicit learning and total adaptation (black lines in Fig. 5-Supplement 2), and a positive statistically significant relationship occurred between explicit learning and total adaptation (green lines in Fig. 5-Supplement 3). Statistically significant relationships were detected using a regression across the 30 participants in each simulation $(p<0.05)$. The bottom row in Fig. 5-Supplement 3, shows the average $R^{2}$ statistic for these linear regressions.

\section{Data availability}

Source data files generated or analyzed during this study, as well as the associated analysis code, are included as supplements to Figures 1-10, as well as their associated Figure Supplements, and have also been deposited in OSF under accession code MZS6A. 

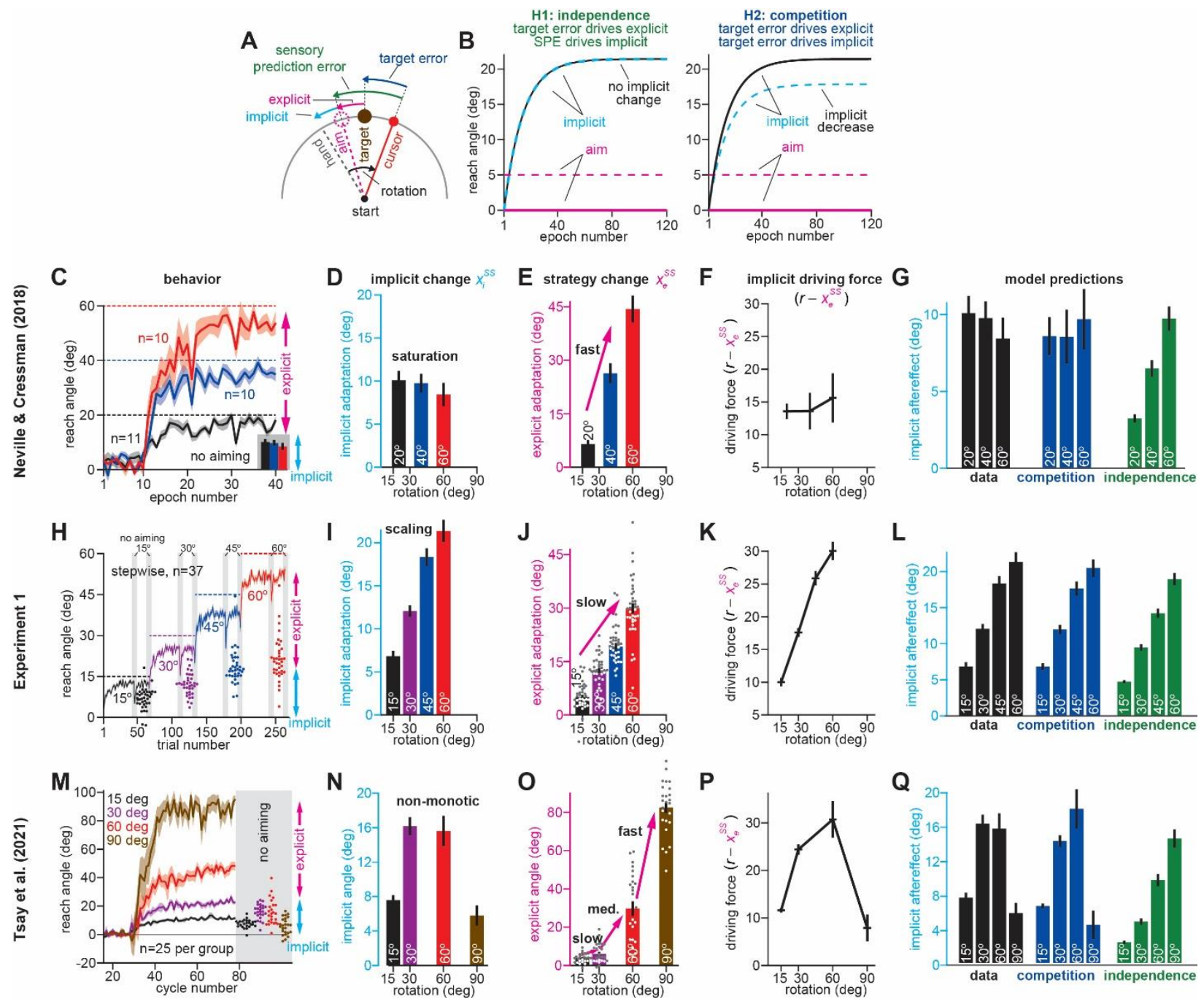

Figure 1. Total implicit learning is shaped by competition with explicit strategy. A. Schematic of visuomotor rotation. Participants move from S to T. Hand path is composed of explicit (aim) and implicit corrections. Cursor path is perturbed by rotation. We explored two hypotheses: prediction error ( $\mathrm{H} 1$, aim vs. cursor) vs. target error ( $\mathrm{H} 2$, target vs. cursor) drives implicit learning. B. Prediction error hypothesis predicts that enhancing aiming (dashed magenta) will not change implicit learning (black vs. dashed cyan) according to the independence equation. Target error hypothesis predicts that enhancing aiming (dashed magenta) will decrease implicit adaptation (black vs. dashed cyan). C. Data reported by Neville and Cressman ${ }^{30}$. Participants were exposed to either a $20^{\circ}, 40^{\circ}$, or $60^{\circ}$ rotation. Learning curves are shown. The "no aiming" inset shows implicit learning measured via exclusion trials at the end of adaptation. Explicit strategy was calculated as the decrease in reach angle during the no aiming period. D. Implicit learning measured during no aiming period in Neville and Cressman yielded a "saturation" phenotype. E. Explicit strategies calculated in Neville \& Cressman dataset by subtracting exclusion trial reach angles from the total adapted reach angle. F. The implicit learning driving force in the competition theory: difference between rotation and explicit learning in Neville and Cressman. G. Implicit learning predicted by the competition and independence models in Neville and Cressman. Models were fit assuming that the implicit learning gain was identical across rotation sizes. H. Experiment 1 . Subjects in the stepwise group $(n=37)$ experienced a $60^{\circ}$ rotation gradually in 4 steps: $15^{\circ}, 30^{\circ}, 45^{\circ}$, and $60^{\circ}$. Implicit learning was measured via exclusion trials (points) twice in each rotation period (gray 'no aiming'). I. Total implicit learning calculated during each 
3101 rotation period in the stepwise group yielded a "scaling" phenotype. J. Explicit strategies were calculated in the 3102 stepwise group by subtracting exclusion trial reach angles from the total adapted reach angle. K. The implicit 3103 learning driving force in the competition theory: difference between rotation and explicit learning in the stepwise 3104 group. L. Implicit learning predicted by the competition and independence models in the stepwise group. Models 3105 were fit assuming that implicit learning gain was constant across rotation size. M. Data reported by Tsay and 3106 colleagues $^{35}$. Participants were exposed to either a $15^{\circ}, 30^{\circ}, 60^{\circ}$, or $90^{\circ}$ rotation. Learning curves are shown. The 3107 "no aiming" inset shows implicit learning measured via exclusion trials at the end of adaptation. N. Implicit 3108 learning measured during no aiming period in Tsay et al. yielded a "non-monotonic" phenotype. O. Explicit strategies calculated in Tsay et al. dataset by subtracting exclusion trial reach angles from the total adapted reach angle. P. Implicit learning driving force in the competition theory: difference between rotation and explicit learning in Tsay et al. Q. Total implicit learning predicted by the competition and independence models in Tsay et al. 3112 Models were fit assuming that the implicit learning gain was identical across rotation sizes. Error bars show mean \pm 3113 SEM, except in the independence predictions in $\mathbf{G}, \mathbf{L}$, and $\mathbf{Q}$; independence predictions show mean and standard 3114 deviation across 10,000 bootstrapped samples. Points in $\mathbf{H}, \mathbf{J}, \mathbf{M}$, and $\mathbf{O}$ show individual participants. 
1. Measuring changes in implicit adaptation after priming the explicit system
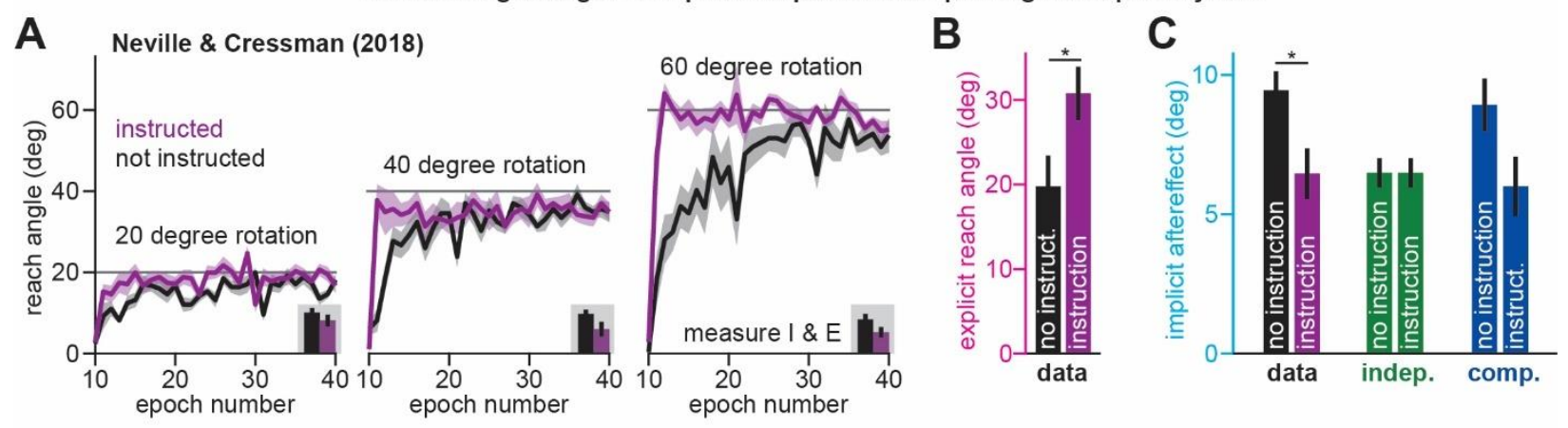

2. Measuring changes in implicit adaptation after decreasing rotation awareness

E
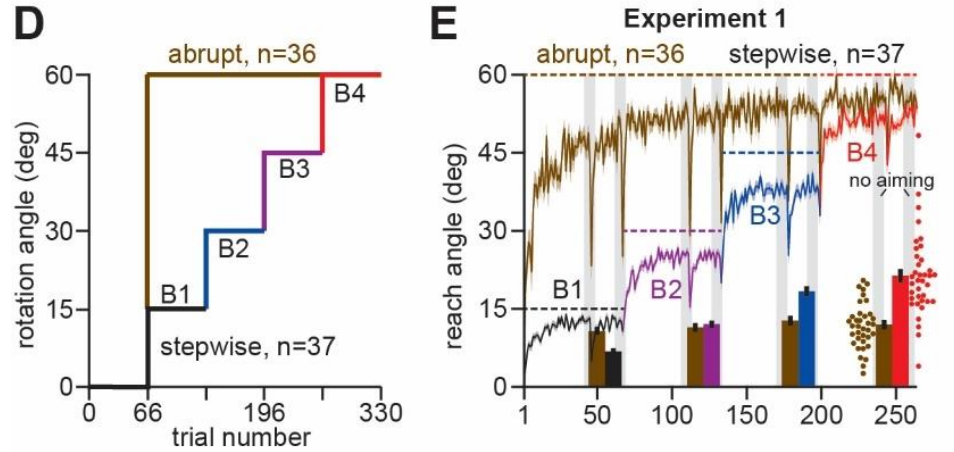

$\mathbf{F}$
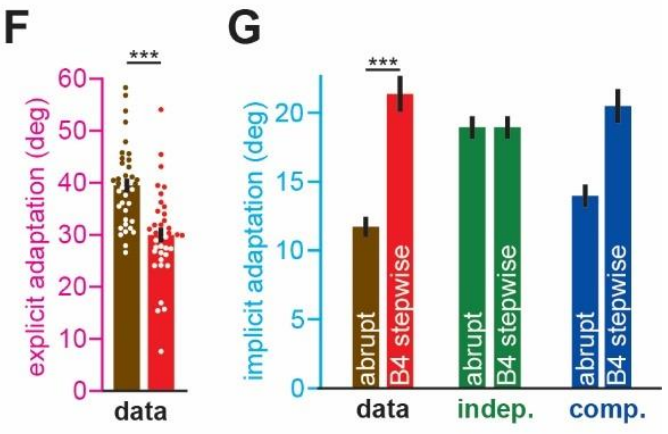

Figure 2. Increases or decreases in explicit strategy change implicit adaptation. A. Neville \& Cressman (2018) tested participants in two conditions: an uninstructed condition (black) and an instructed condition (purple) where subjects were briefed about the upcoming rotation and its solution. Instruction increased the adaptation rate across three rotation sizes: $20^{\circ}, 40^{\circ}$, and $60^{\circ}$. Insets in gray shaded area show implicit adaptation measured via exclusion trials at the end of adaptation. B. Here we show the average strategy across all rotation sizes in the instructed (black) and uninstructed (purple) conditions. Explicit strategy was calculated by subtracting implicit learning (exclusion trials) from total adaptation. Instruction increased explicit strategy use. C. The data show implicit adaptation averaged across all 3 rotation sizes. The independent (SPE learning) and competition (target error learning) models were fit to these data assuming that implicit error sensitivity and retention were identical across rotation sizes and instruction conditions (i.e., identical $a_{i}$ and $b_{i}$ across all 6 groups). Error bars for model predictions refer to mean and standard deviation across 10,000 bootstrapped samples. D. In Experiment 1 we tested participants in either an abrupt condition or a stepwise (gradual) condition. Here we show the rotation schedule. E. Here we show learning curves in the abrupt and stepwise conditions in Experiment 1. Bars show implicit adaptation measured during each rotation period (4 blocks total) via exclusion trials. Individual learning measures are shown in the terminal $60^{\circ}$ learning period for both groups (points at bottom-right). F. We calculated explicit strategies during the terminal $60^{\circ}$ learning period by subtracting implicit learning measures from total adaptation (mean over last 20 trials). Gradual onset reduced explicit strategy use. G. The data show total implicit learning measured in the $60^{\circ}$ rotation period. The competition (blue) and independence (green) models were fit to the data assuming that the implicit learning parameters were the same across the abrupt and stepwise groups. Error bars for the model show the mean and standard deviation across 1000 bootstrapped samples. Statistics in $\mathbf{B}$, $\mathbf{F}$, and $\mathbf{G}$ denote a two-sample t-test: ${ }^{*} \mathrm{p}<0.05,{ }^{* *} \mathrm{p}<0.001$. Error bars in $\mathbf{A}, \mathbf{B}, \mathbf{C}$ (data), E, F, and $\mathbf{G}$ (data) denote

SEM.

Points

in

E and

F show

individual

participants. 
A

Experiment 2, No PT Limit

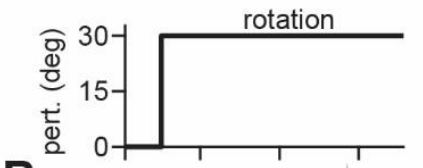

B
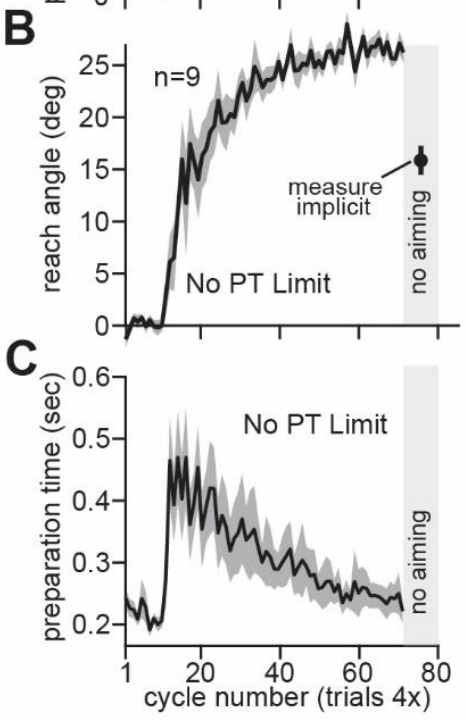

\section{$F$}

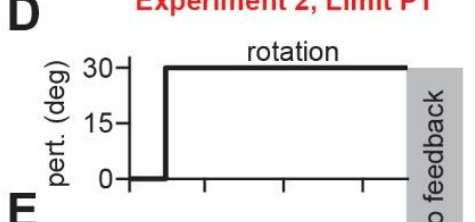

E
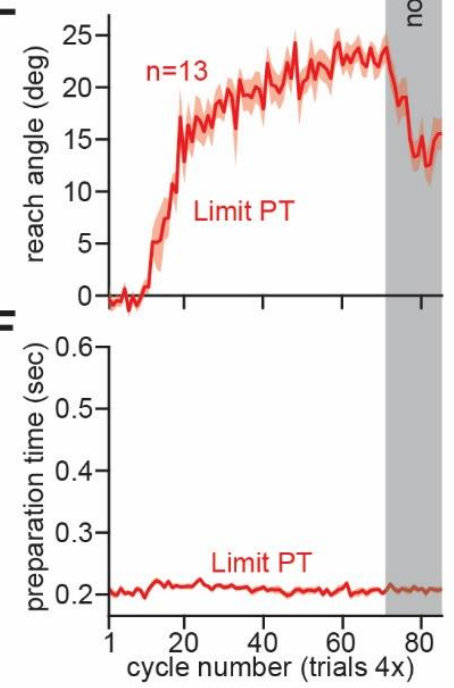

H

Experiment 3, No PT Limit

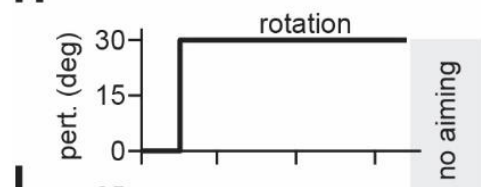

I
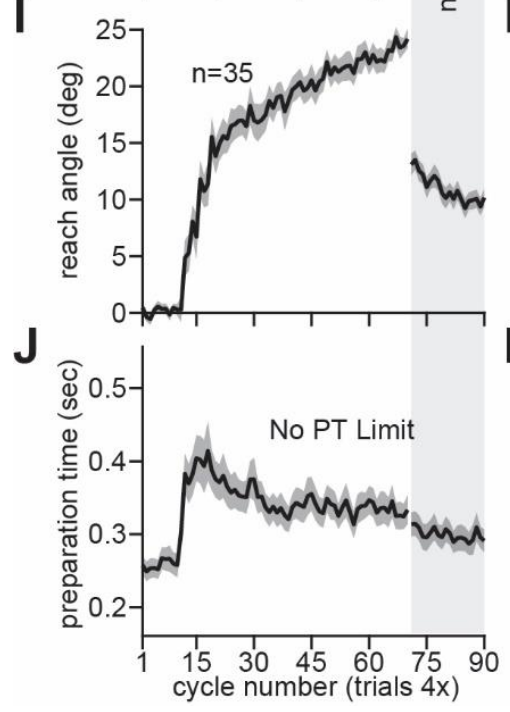

Figure 3. Strategy suppresses implicit learning across individual participants. A-C. In Experiment 2, participants in the No PT Limit (no preparation time limit) group adapted to a $30^{\circ}$ rotation. The paradigm is shown in $\mathbf{A}$. The learning curve is shown in B. Implicit learning was measured via exclusion trials (no aiming). Preparation time is shown in $\mathbf{C}$ (movement start minus target onset). D-F. Same as in A-C, but in a limited preparation time condition (Limit PT). Participants in the Limit PT group had to execute movements with restricted preparation time (F). The task ended with a prolonged no visual feedback period where memory retention was measured (E, gray region). $\mathbf{G}$. 
Total implicit and explicit adaptation in each participant in the No PT Limit condition (points). Implicit learning measured during the terminal no aiming probe. Explicit learning represents difference between total adaptation (last 10 rotation cycles) and implicit probe. The black line shows a linear regression. The blue line shows the theoretical relationship predicted by the competition equation which assumes implicit system adapts to target error. The parameters for this model prediction (implicit error sensitivity and retention) were measured in the Limit PT group. $\mathbf{H}-\mathrm{J}$. In Experiment 3, participants adapted to a $30^{\circ}$ rotation using a personal computer in the No PT Limit condition. The paradigm is shown in $\mathbf{H}$. The learning curve is shown in I. Implicit learning was measured at the end of adaptation over a 20-cycle period where participants were instructed to reach straight to the target without aiming and without feedback (no aiming seen in I). We measured explicit adaptation as difference between total adaptation and reach angle on first no aiming cycle. We measured "early" implicit aftereffect as reach angle on first no aiming cycle. We measured "late" implicit aftereffect as mean reach angle over last 15 no aiming cycles. KM. Same as in H-J, but for a Limit PT condition. N. Explicit adaptation measured in the No PT Limit condition in Experiment 2 (E2), No PT Limit condition in Experiment (E3, black), and Limit PT condition in Experiment 3 (E3, red). O. Late implicit learning in the Experiment 3 No PT Limit group (No Lim.) and Experiment 3 Limit PT group (PT Limit). P. Correspondence between late implicit learning and explicit strategy in the Experiment 3 No PT Limit group. Q. Same as in $\mathbf{G}$ but where model parameters are obtained from the Limit PT group in Experiment 3, and points represent subjects in the No PT Limit group in Experiment 3. Early implicit learning is used. Throughout all insets, error bars indicate mean \pm SEM across participants. Statistics in $\mathbf{N}$ and $\mathbf{O}$ are two-sample t-tests: $n$.s. means $p>0.05, * * * p<0.001$. 

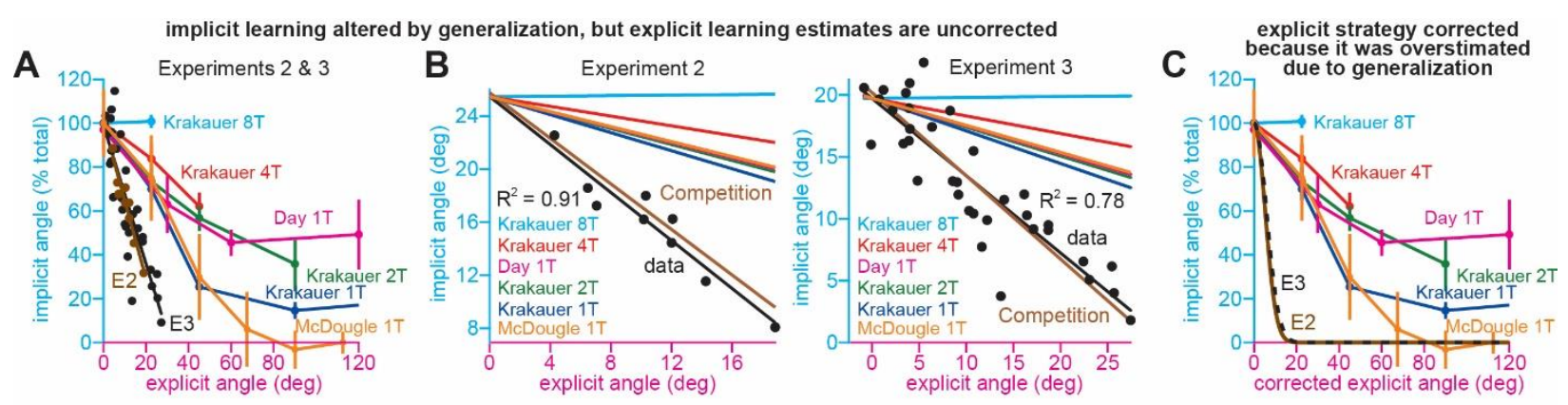

D
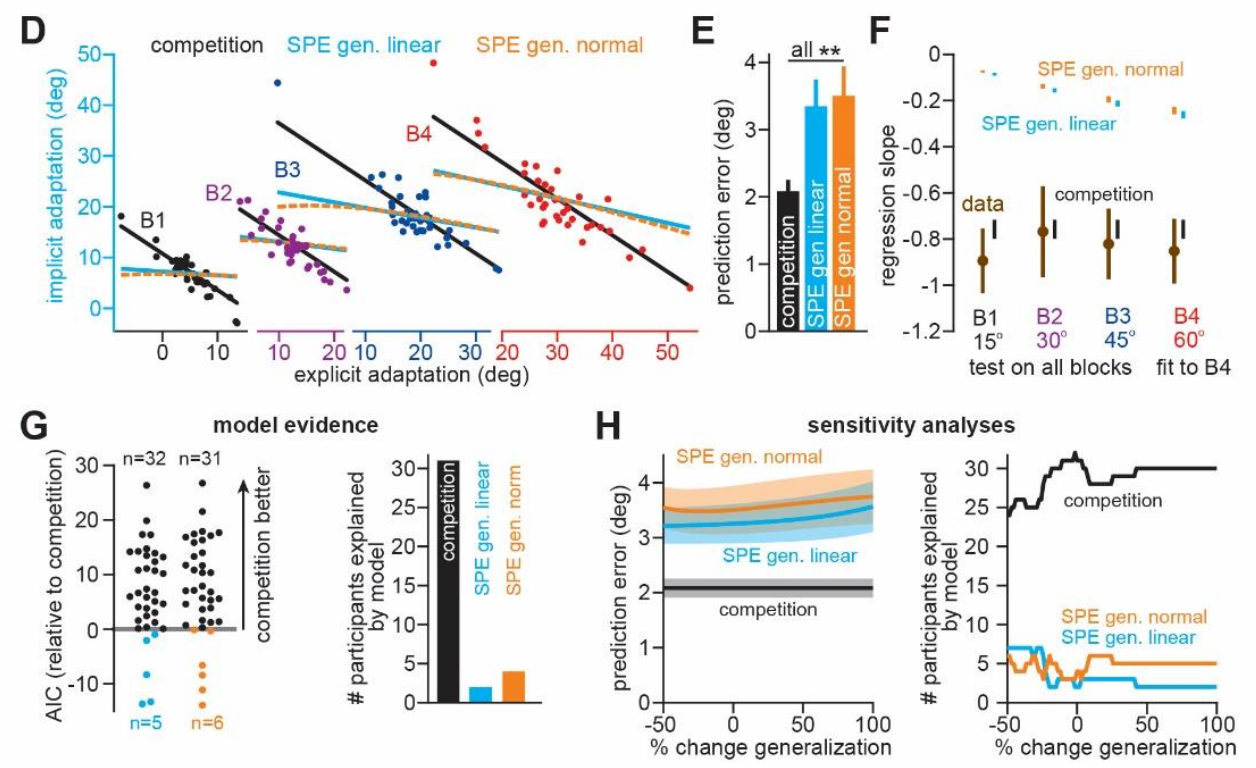

Figure 4. Correlations between implicit and explicit learning are consistent with competition, not SPE generalization. A. Aim-centered generalization could create the illusion that implicit and explicit systems compete. To evaluate this possibility, we compared the implicit-explicit relationship in Exps. $2 \& 3$ to generalization curves reported in Krakauer et al. (2000) ${ }^{47}$, Day et al. $(2016)^{49}$, and McDougle et al. (2017) ${ }^{50}$. The 1T, 2T, 4T, and 8T labels correspond to the number of adaptation targets in Krakauer et al. The gold McDougle et al. curve is particularly relevant because the authors controlled aiming direction on generalization trials and counterbalanced $\mathrm{CW}$ and CCW rotations. Data in Exps. 2\&3 are shown overlaid on the inset. Implicit learning declined about 300\% more rapidly with increases in re-aiming that that observed by Day et al. The solid black and brown lines show the competition theory predictions. Implicit learning in Experiments 1 and 2 was normalized to its theoretical maximum, reached when re-aiming is equal to zero. The value used to normalize was determined via linear regression (25.5 in Exp. 2, $19.7^{\circ}$ in Exp. 3). B. Same as in A, but without normalizing implicit learning. Generalization curves were converted to degrees by multiplying the curves in $\mathbf{A}$ by the max. implicit learning value in Exp. $2\left(25.5^{\circ}\right)$ or Exp. $3\left(19.7^{\circ}\right)$. C. The comparisons in A and B are not correct. Under the generalization hypothesis, each data point's explicit strategy needs to be corrected according to generalization. This inset shows the true implicit-explicit generalization curve that would be required to produce the data in $\mathbf{A}$ and $\mathbf{B}$. The E2 and E3 lines show the Exp. 2 and Exp. 3 curves. D. Points show implicit and explicit learning measured in the stepwise individual participants studied in Exp. 1 ( $\mathrm{B} 1$ is $15^{\circ}$ period, $\mathrm{B} 2$ is $30^{\circ}$ period, $\mathrm{B} 3$ is $45^{\circ}$ period, and $\mathrm{B} 4$ is $60^{\circ}$ period). 3 models were fit to participant data in the $60^{\circ}$ period. Competition model fit is shown in black. A linear generalization (SPE gen. linear) with slope set by McDougle et al. is shown in cyan. A Gaussian generalization (SPE gen. normal) with width set by McDougle et al. is shown in gold. Since models were fit to B4 data, the B1, B2, and 
3186 the held-out $15^{\circ}, 30^{\circ}$, and $45^{\circ}$ periods in D. F. Linear regressions fit to each rotation block in D. Brown points and 3187 lines (data) show the regression slope and $95 \% \mathrm{Cl}$. The black (competition), cyan (SPE gen. linear), and gold (SPE 3188 gen. normal) are model predictions where lines are 95\% Cls estimated via bootstrapping. G. All 3 models in D-F 3189 were fit to individual participant behavior in the stepwise group. At left, the AIC for each model is compared to 3190 that of the competition model. At right, the total number of subjects best captured by each model is shown. $\mathbf{H}$. 3191 Same as $\mathbf{E}$ and $\mathbf{G}$ but where the generalization width was varied in a sensitivity analysis. We tested values between 3192 one-half the McDougle et al. generalization curve $(-50 \%)$ and twice the McDougle et al. generalization curve 3193 (+100\%). Error bars in E show mean \pm SEM. Statistics in E are post-hoc tests following one-way rm-ANOVA: $3194 * *$ * $<0.01$. 
Simulation: SPE learning model: implicit system exhibits variable learning, explicit system compensates
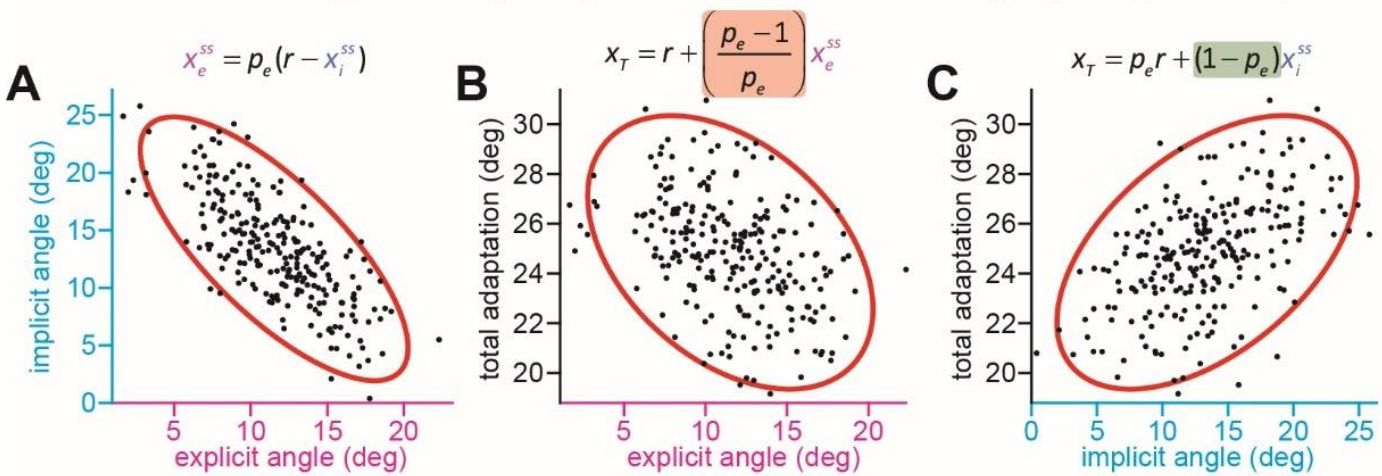

Simulation: competition model: explicit system exhibits variable learning, implicit system compensates
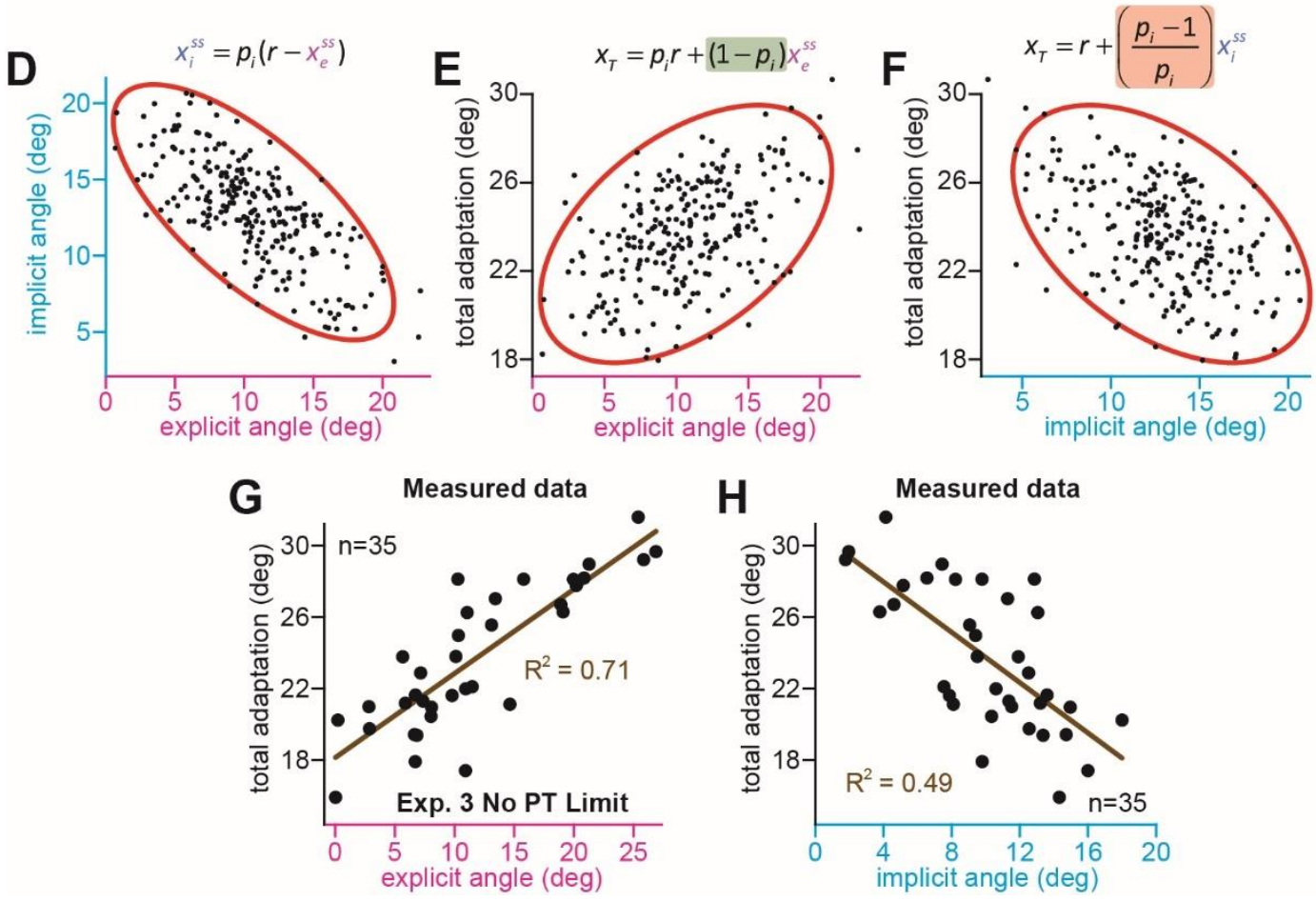

Figure 5. Implicit-explicit correlations with total adaptation match the competition theory. The competition equation states that $x_{i}^{s s}=p_{i}\left(r-x_{e}^{s s}\right)$, where $p_{i}$ is a scalar learning gain depending on $a_{i}$ and $b_{i}$. The competition between steady-state implicit $\left(x_{i}^{s s}\right)$ and explicit $\left(x_{e}^{s s}\right)$ adaptation predicted by this model is simulated in $\mathbf{D}$ across 250 hypothetical participants. The model $p_{i}$ is fit to data in Experiment 3. Total learning is given by $x_{T}^{s s}=x_{i}^{s s}+x_{e}^{s s}$. These two equations can be used to derive expressions relating total learning $\left(x_{T}^{s s}\right)$ to steady-state implicit $\left(x_{i}^{s s}\right)$ and explicit $\left(x_{e}^{s s}\right.$ ) learning. In $\mathbf{E}$ we show that the competition theory predicts a positive relationship between explicit learning and total adaptation (equation at top derived in Methods, green denotes a positive gain). In $\mathbf{F}$ we show that the competition theory predicts a negative relationship between implicit learning and total adaptation (equation at top derived in Methods, red shading denotes negative gain). In A-C we consider an alternative model. Suppose that implicit learning is immune to explicit strategy and varies independently across participants. This is equivalent to the SPE learning model. But in this case, the explicit system could respond to variability in implicit learning via another competition equation: $x_{e}^{s s}=p_{e}\left(r-x_{i}^{s s}\right)$. Here $p_{e}$ is an explicit learning gain (must be less than 1 to yield a stable system). In $\mathbf{A}$ we show the negative relationship between implicit and explicit adaptation predicted by this alternate SPE learning model. In B we show that when the explicit system responds to implicit 
3210 variability (SPE learning) there is a negative relationship between total adaptation and explicit strategy. The 3211 equation at top is derived in Methods. In C, we show that the SPE learning model will yield a positive relationship 3212 between implicit learning and total adaptation. Equation at top derived in Methods. G. We measured the 3213 relationship between explicit strategy and total adaptation in Exp. 3 (No PT Limit group). Total learning exhibits a 3214 positive correlation with explicit strategy. H. Same concept as in G, but here we show the relationship between 3215 total learning and implicit adaptation. The patterns in $\mathbf{G}$ and $\mathbf{H}$ are consistent with the competition theory 

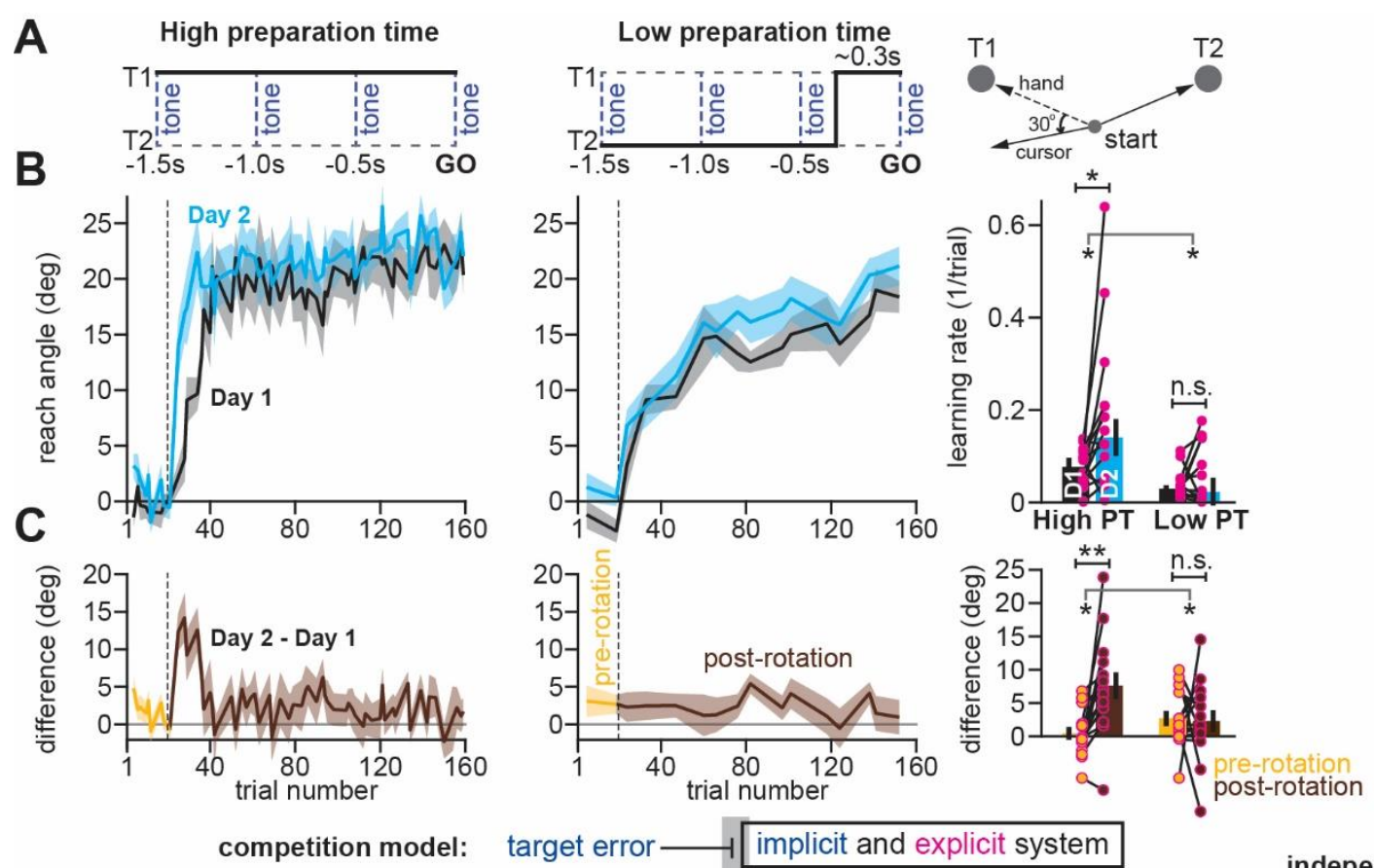

target error $\longrightarrow$ implicit and explicit system
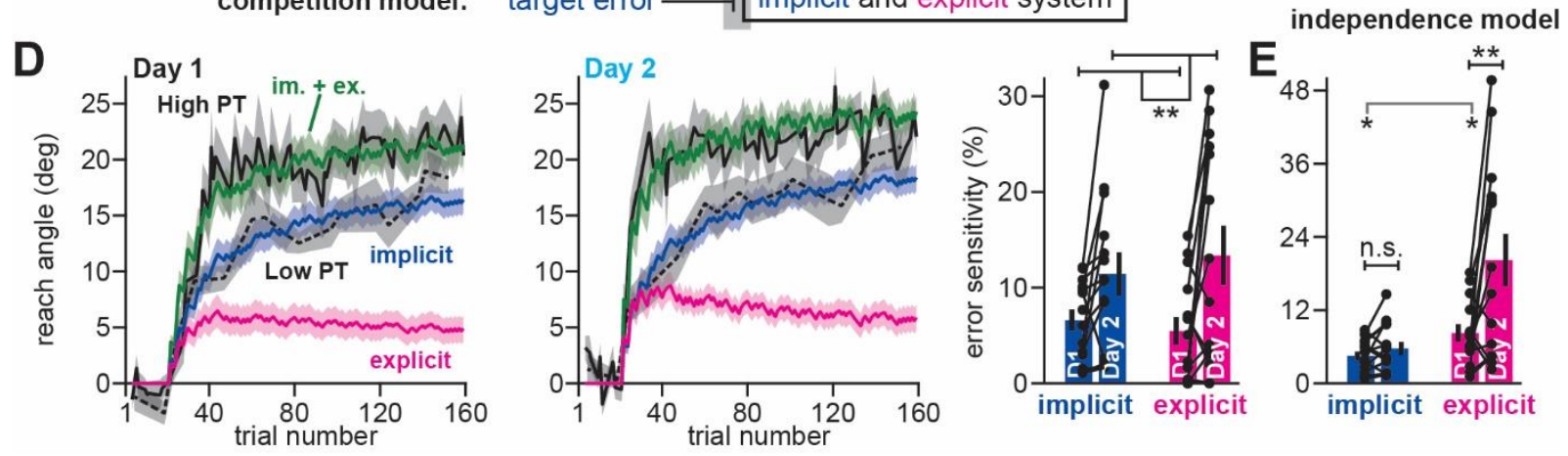

Figure 6. Competition predicts changes in implicit error sensitivity without changes in implicit learning rate. A. Haith and colleagues ${ }^{13}$ instructed participants to reach to Targets T1 and T2 (right). Participants were exposed to a $30^{\circ}$ visuomotor rotation at Target $\mathrm{T} 1$ only. Participants reached to the target coincident with a tone. Four tones were played with a $500 \mathrm{~ms}$ inter-tone-interval. On most trials (80\%) the same target was displayed during all four tones (left, High preparation time or High PT). On some trials (20\%) the target switched approximately $300 \mathrm{~ms}$ prior to the fourth tone (middle, Low preparation time or Low PT). B. On Day 1, participants adapted to a $30^{\circ}$ visuomotor rotation (Block 1, black) followed by a washout period. On Day 2, participants again experienced a $30^{\circ}$ rotation (Block 2, blue). At left, we show the reach angle expressed on High PT trials during Blocks 1 and 2. Dashed vertical line shows perturbation onset. At middle, we show the same but for Low PT trials. At right, we show learning rate on High and Low PT trials, during each block. C. As an alternative to the rate measure shown at right in $\mathbf{B}$, we calculated the difference between reach angle on Blocks 1 and 2. At left and middle, we show the learning curve differences for High and Low PT trials, respectively. At right, we show difference in learning curves before and after the rotation. 'Pre-rotation' shows the average of Day 2 - Day 1 prior to rotation onset. 'Post-rotation' shows the average of Day 2 - Day 1 after rotation onset. D. We fit a state-space model to the learning curves in Blocks 1 and 2 assuming that target errors drove implicit adaptation. Low PT trials captured the implicit system (blue). High PT trials captured the sum of implicit and explicit systems (green). Explicit trace (magenta) is the difference between the High and Low PT predictions. At right, we show error sensitivities predicted by the model. 
3236 predicted error sensitivities are shown. Error bars across all insets show mean \pm SEM, except for the learning rate 3237 in $\mathbf{B}$ which displays the median. Two-way repeated-measures ANOVA were used in B, C, D, and E. For B and C, 3238 exposure number and preparation time condition were main effects. For $\mathbf{D}$ and $\mathbf{E}$ exposure number and learning 3239 system (implicit vs explicit) were main effects. Significant interactions in B, C, and E prompted follow-up one-way 3240 repeated-measures ANOVA (to test simple main effects). Statistical bars where two sets of asterisks appear (at left 3241 and right) indicate interactions. Statistical bars with one centered set show main effects or simple main effects.

3242 Statistics: n.s. means $p>0.05, * p<0.05, * * p<0.01$. 
A
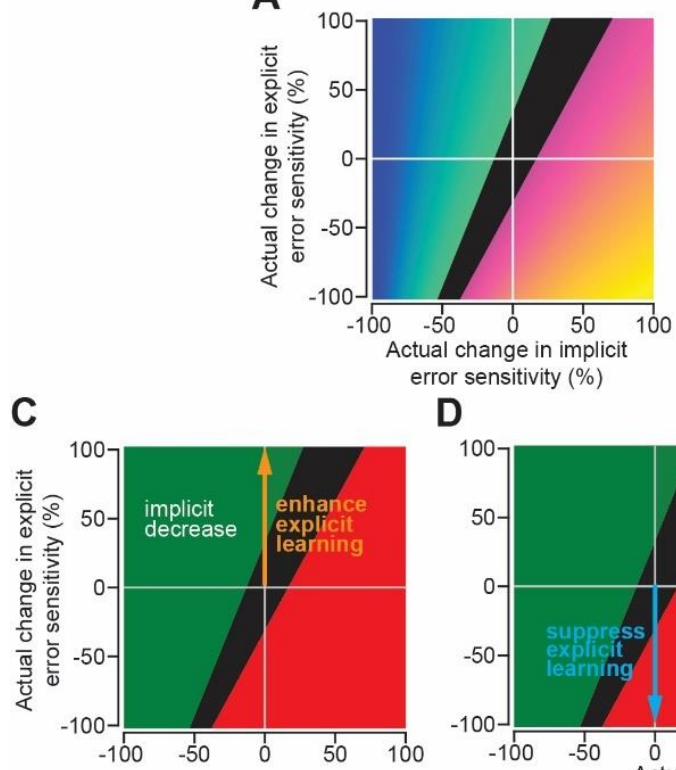

D
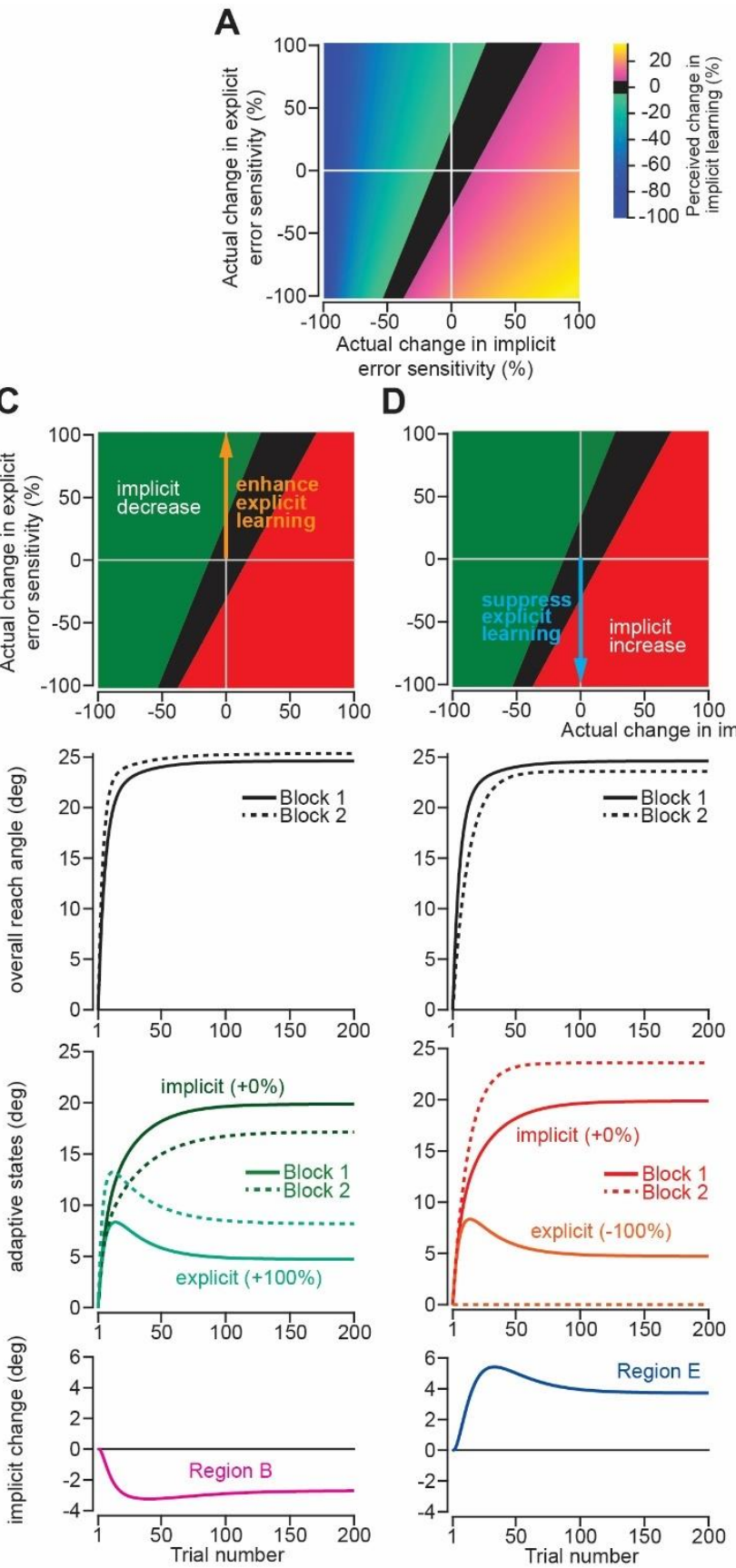

E
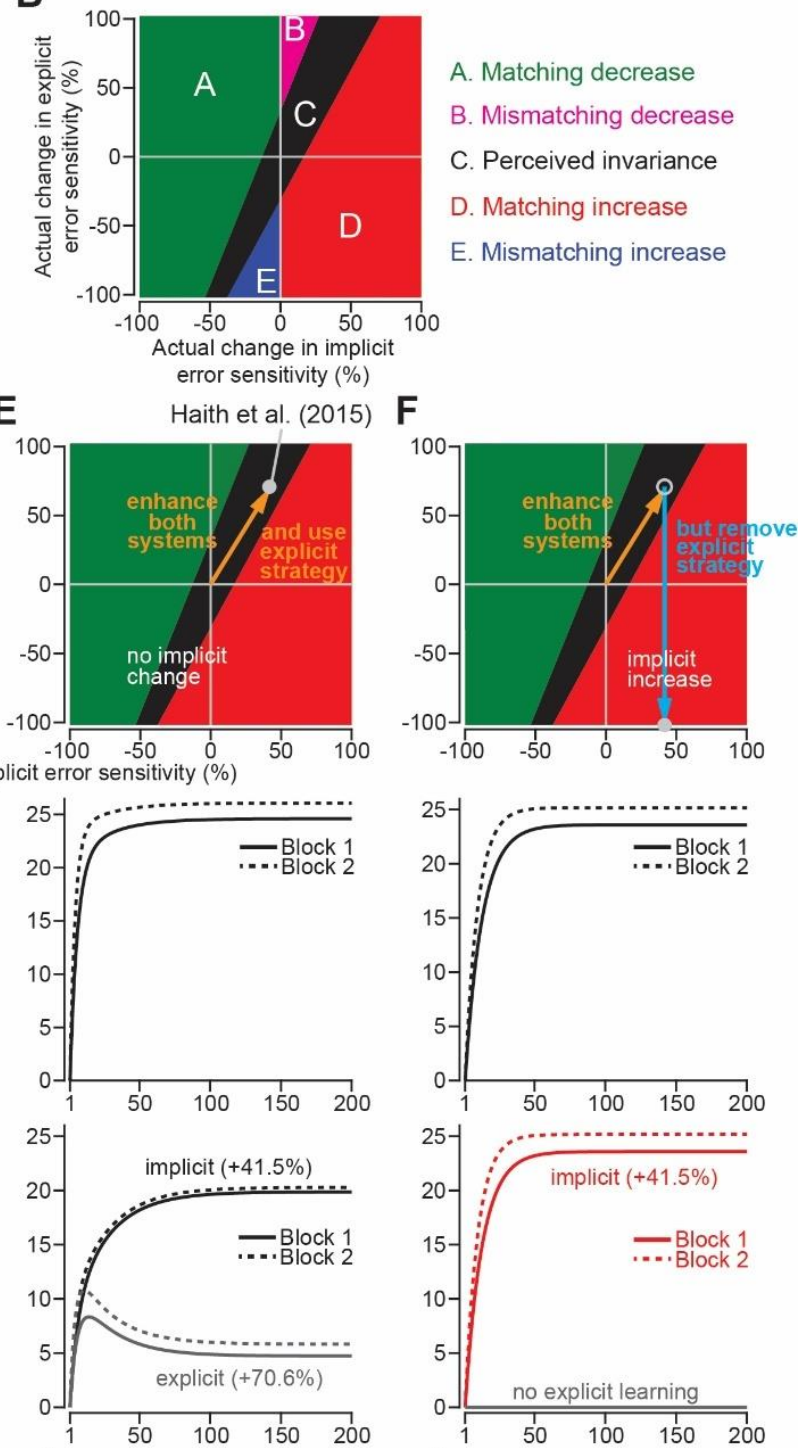

B. Mismatching decrease

C. Perceived invariance

D. Matching increase

E. Mismatching increase
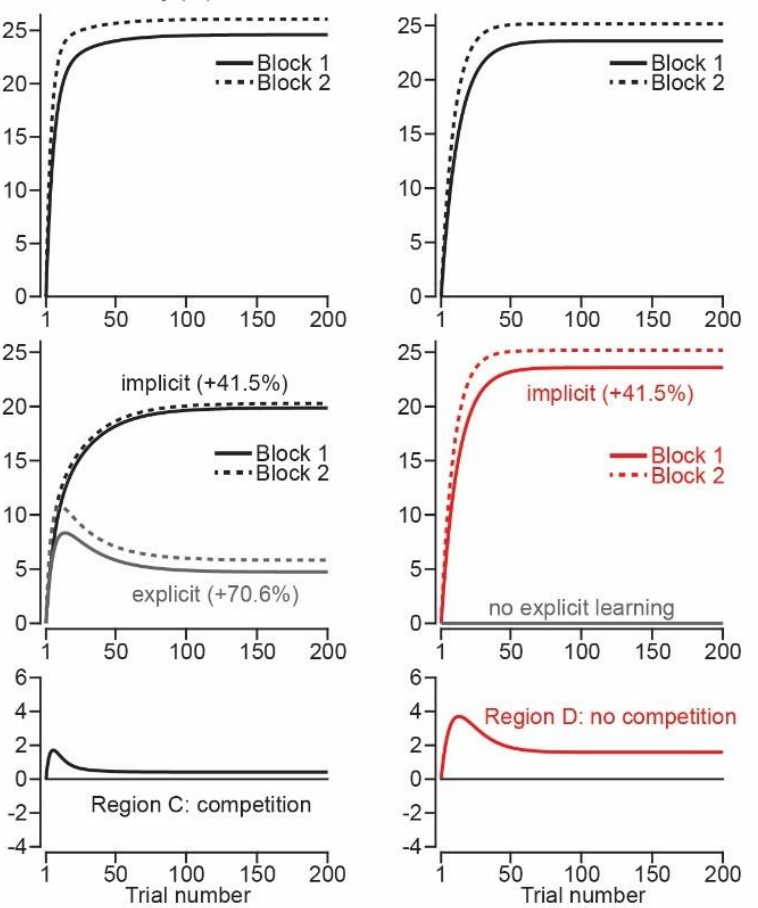

A. Matching decrease

Figure 7. Changes in implicit adaptation depend on both implicit and explicit error sensitivity. A. Here we depict the competition map. The $x$-axis shows change in implicit error sensitivity between reference and test conditions. The $y$-axis shows change in explicit error sensitivity. Colors indicate the percent change in implicit adaptation (measured at steady-state) from the reference to test conditions. Black region denotes an absolute change less than 5\%. The map was constructed with Eq. (8). B. The map can be described in terms of 5 different regions. In Region A (matching increase), implicit error sensitivity and total implicit adaption both increase in test condition. Region $D$ is same, but for decreases in error sensitivity and total adaptation. In Region B (mismatching decrease) implicit learning decreases though its error sensitivity is higher or same. In Region E (mismatching increase), implicit learning increases though its error sensitivity is lower or same. Region C shows a perceived invariance where implicit adaptation changes less than 5\%. C. Row 1: effect of enhancing explicit learning. Row 2: total 
3254 learning increases. Row 3: implicit and explicit learning shown in Blocks 1 and 2, where only difference is $100 \%$ 3255 increase in explicit error sensitivity. Row 4: change in implicit learning (Block 1 to 2). D. Row 1: effect of 3256 suppressing explicit learning. Row 2: total learning decreases. Row 3: implicit and explicit learning shown in Blocks 32571 and 2, where explicit error sensitivity decreases 100\%. Row 4: implicit learning change (Block 1 to 2). E. Row 1: 3258 model simulation for Haith et al. ${ }^{13}$ Row 2: Total learning increases. Row 3: implicit and explicit learning during 3259 Blocks 1 and 2 where implicit error sensitivity increases by $41.5 \%$ and explicit error sensitivity increases by $70.6 \%$. 3260 Row 4: negligible change in implicit learning (Block 1 to 2). F. Same as in E except here explicit strategy is 3261 suppressed during Blocks 1 and 2. 

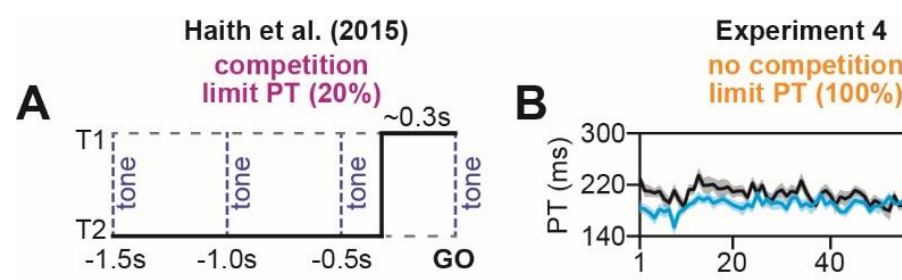

Experiment 4

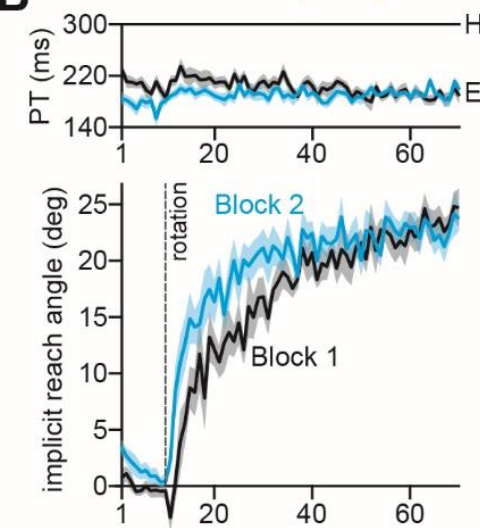

Haith et al. (2015)
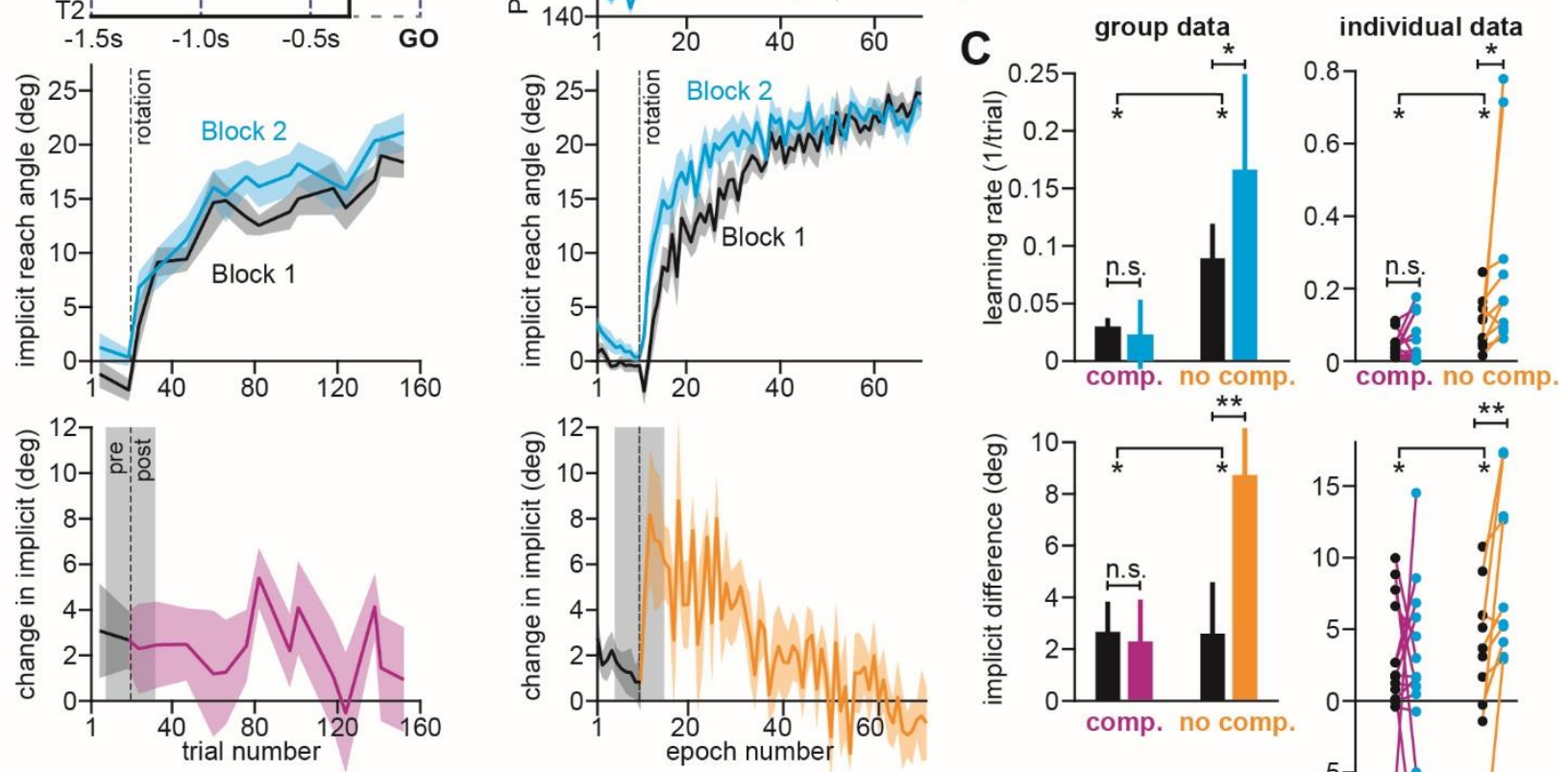

Figure 8. Removing explicit strategy reveals savings in implicit adaptation. A. Top: Low preparation time (Low PT) trials in Haith and colleagues ${ }^{13}$ used to isolate implicit learning. Middle: learning during Low PT in Blocks 1 and 2. Bottom: difference in Low PT learning between Blocks 1 and 2. B. Similar to A, but here (Experiment 4) explicit learning was suppressed on every trial, as opposed to only $20 \%$ of trials. To suppress explicit strategy, we restricted reaction time on every trial. The reaction time during Blocks 1 and 2 is shown at top. At middle, we show how participants adapted to the rotation under constrained reaction time. At bottom, we show the difference between the learning curves in Blocks 1 and 2. These two periods were separated by washout cycles with veridical feedback (not shown). C. Here we measured savings in Haith et al. (20\% of trials had reaction time limit) and Experiment 3 (100\% of trials had reaction time limit). Top row: we quantify savings by fitting an exponential curve to each learning curve. Data are the rate parameter associated with the exponential. Left column shows group-level data (median). Right column shows individual participants. Bottom row: we quantify savings by comparing how Blocks 1 and 2 differed before perturbation onset (black), and after perturbation onset (purple and yellow). At left, error bars show mean \pm SEM. At right, individual participants are shown. Error bars in $\mathbf{A}$ and $\mathbf{B}$ indicate mean \pm SEM. Statistics in C show mixed-ANOVA (exposure number is within-subject factor, experiment type is between-subject factor). Significant interactions were observed both in rate (top) and angular (bottom) savings measure. Follow-up simple main effects were assessed via one-way repeated-measures ANOVA. Statistical bars where two sets of asterisks appear (at left and right) indicate interactions. Statistical bars with a centered set show simple main effects.

Statistics:

n.s.

means

$p>0.05$,

${ }^{*} \mathrm{p}<0.05$,

$* * \mathrm{p}<0.01$. 
A
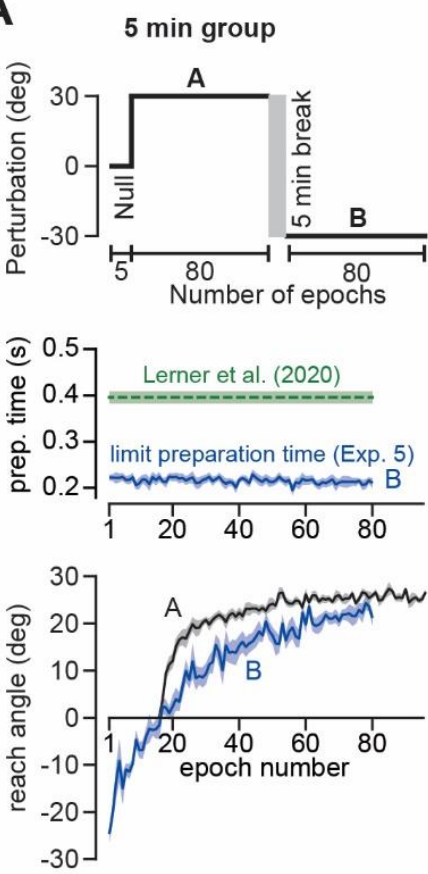

B $24 \mathrm{hr}$ group
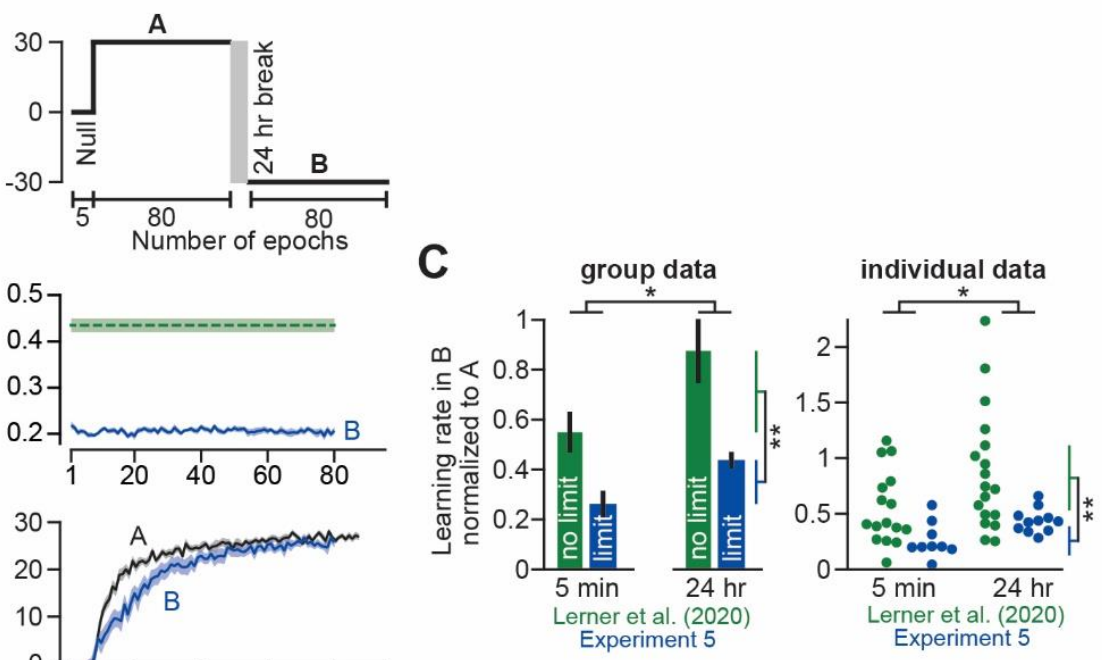

Experiment 5
3281

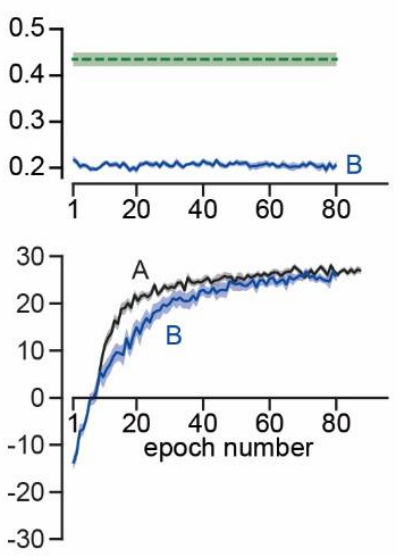

Figure 9. Removing explicit strategy reveals anterograde interference in implicit adaptation. A. Top: participants were adapted to a $30^{\circ}$ rotation $(A)$. Following a 5 -minute break, participants were then exposed to a $-30^{\circ}$ rotation (B). This A-B paradigm was similar to that of Lerner \& Albert et al. ${ }^{19}$. Middle: to isolate implicit adaptation, we imposed strict reaction time constraints on every trial. Under these constraints, reaction time (blue) was reduced by approximately $50 \%$ over that observed in the self-paced condition (green) studied by Lerner \& Albert et al. ${ }^{19}$ Bottom: learning curves during $A$ and $B$ in Experiment 5; under reaction time constraints, the interference paradigm produced a strong impairment in the rate of implicit adaptation. To compare learning during $A$ and $B, B$ period learning was reflected across $y$-axis. Furthermore, the curves were temporally aligned such that an exponential fit to the A period and exponential fit to the B period intersected when the reach angle crossed $0^{\circ}$. This alignment visually highlights differences in the learning rate during the $A$ and $B$ periods. B. Here we show the same analysis as in A but when exposures A and B were separated by 24 hours. C. To measure the amount of anterograde interference on the implicit learning system, we fit an exponential to the $A$ and $B$ period behavior. Here we show the B period exponential rate parameter divided by the A period rate parameter (values less than 1 indicate a slowing of adaptation). At left, group-level statistics are shown. At right, individual participants are shown. Data in the Limit PT (limited preparation time) condition in Experiment 5 are shown in blue. Data from Lerner \& Albert et al. (no preparation time limit) are shown in green. A two-way ANOVA was used to test for differences in interference (preparation time condition (i.e., experiment type) was one between-subject factor, time-elapsed between exposures ( $5 \mathrm{~min}$ vs $24 \mathrm{hr}$ ) was the other between-subject factor). Statistical bars indicate each main effect. Statistics: ${ }^{*} p<0.05,{ }^{* *} p<0.01$. Error bars in each inset show mean \pm SEM. 

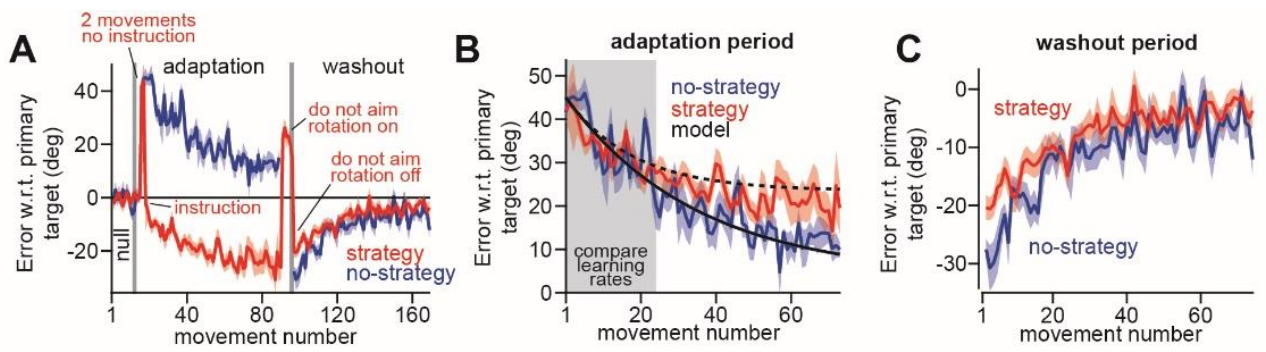

D

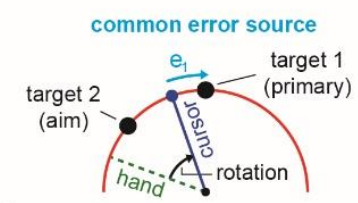

E
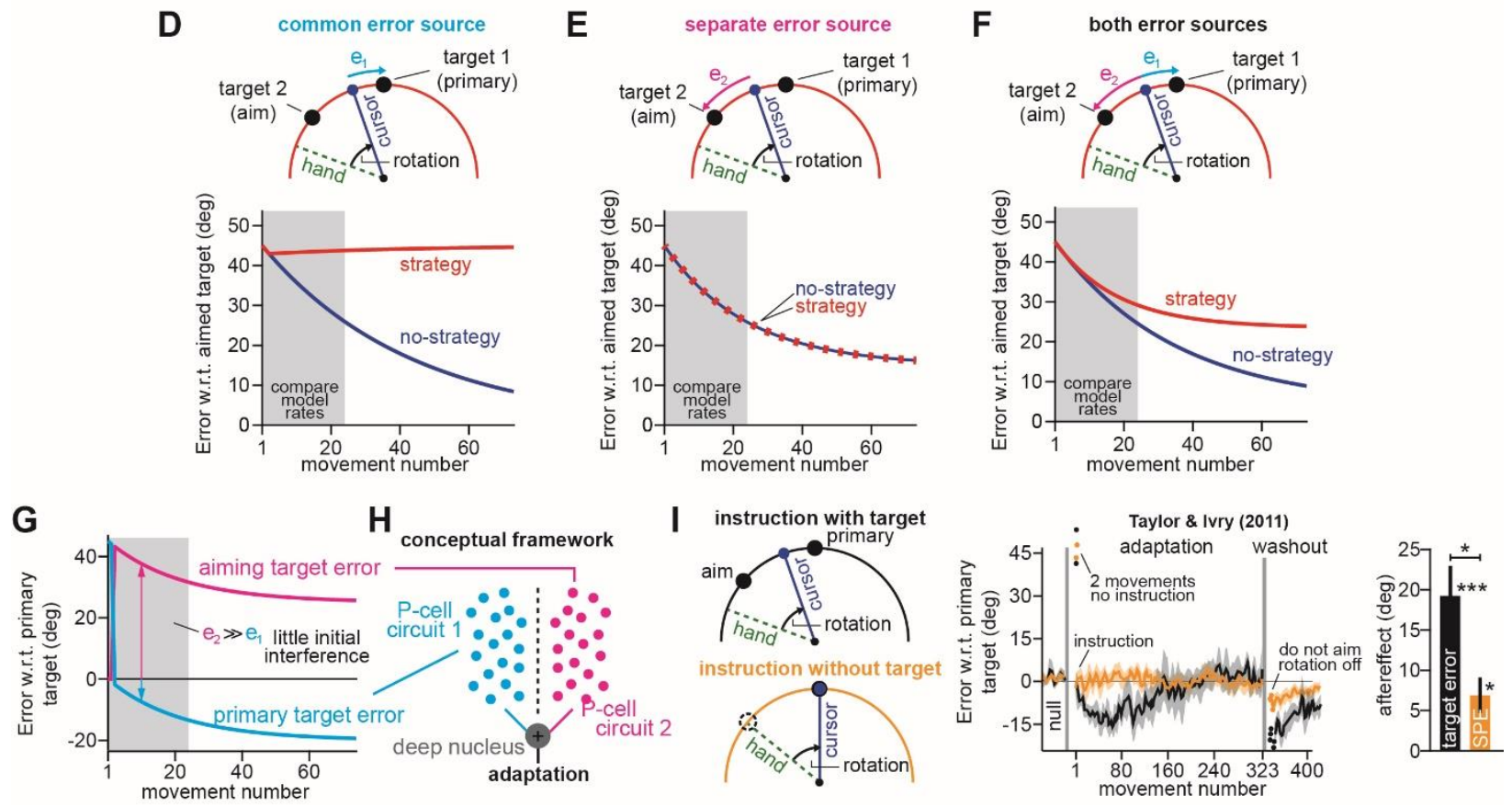

Figure 10. Two visual targets create two implicit error sources. A. Data reported in Mazzoni and Krakauer ${ }^{2}$. Blue shows error between primary target and cursor during adaptation and washout. Red shows the same, but in a strategy group that was instructed to aim to a neighboring target (instruction) to eliminate target errors, once participants experienced two large errors ( 2 cycles no instruction). B. The error between the cursor and the aimed target during the adaptation period. These curves are the same as in $\mathbf{A}$ except we use the aimed target rather than primary target, so as to better compare learning curves across groups. C. The washout period reported in $\mathbf{A}$. Here error is relative to primary target, though in this case aimed and primary targets are the same. D. We modeled behavior when implicit learning adapts to primary target errors $e_{1}$. Note that the no-strategy learning group resembles data. However, strategy learning exhibits no drift because the implicit system has zero error. Note here that the primary target error of $0^{\circ}$ is a $45^{\circ}$ aimed target error in the strategy group. E. Similar to D, except here the implicit system adapts to errors between the cursor and aimed target, termed $e_{2}$. F. In this model, the strategy group adapts to both the primary target error and the aimed target error ( $e_{1}$ and $e_{2}$ at top). The no-strategy group adapts only to the primary target error. Learning parameters are identical across groups. G. We show how aiming target and primary target errors evolve in the strategy group in F. H. A potential neural substrate for implicit learning. The primary target error and aiming target error engage two different sub-populations of Purkinje cells in the cerebellar cortex. These two implicit learning modules combine at the deep nucleus. I. Data reported in Taylor and Ivry ${ }^{8}$. Before adaptation, subjects were taught to re-aim their reach angles. In the "instruction with target" group, participants re-aimed during adaptation with the aid of neighboring aiming targets (top-left). In the "instruction without target" group, participants re-aimed during adaptation without any aiming targets, solely based on the remembered instruction from the baseline period. The middle shows learning curves. In both groups, 
3324 the first 2 movements were uninstructed, resulting in large errors (2 movements no instruction). Note in the 3325 "instruction with target" group, there is an implicit drift as in A, but participants eventually reverse this by 3326 changing explicit strategy. There is no drift in the "instruction without target" group. At right, we show the implicit 3327 aftereffect measured by telling participants not to aim (first no feedback, no aiming cycle post-adaptation). 3328 Greater implicit adaptation resulted from physical target. Error bars show mean \pm SEM. Statistics: ${ }^{*} p<0.05$, $3329 * * * p<0.001$. 
3 possible scenarios (1) strategies grow at same rate as rotation
3331

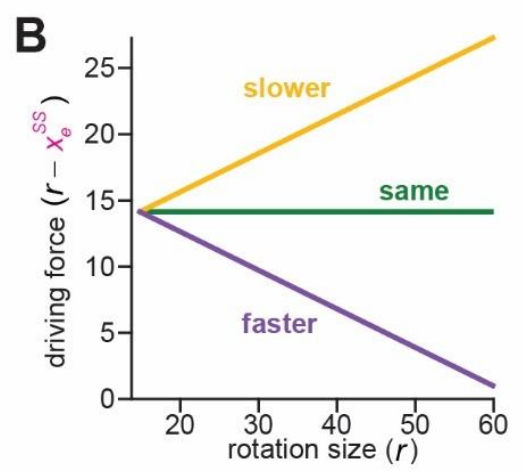

(2) strategies grow at slower rate than rotation
(3) strategies grow at faster rate than rotation
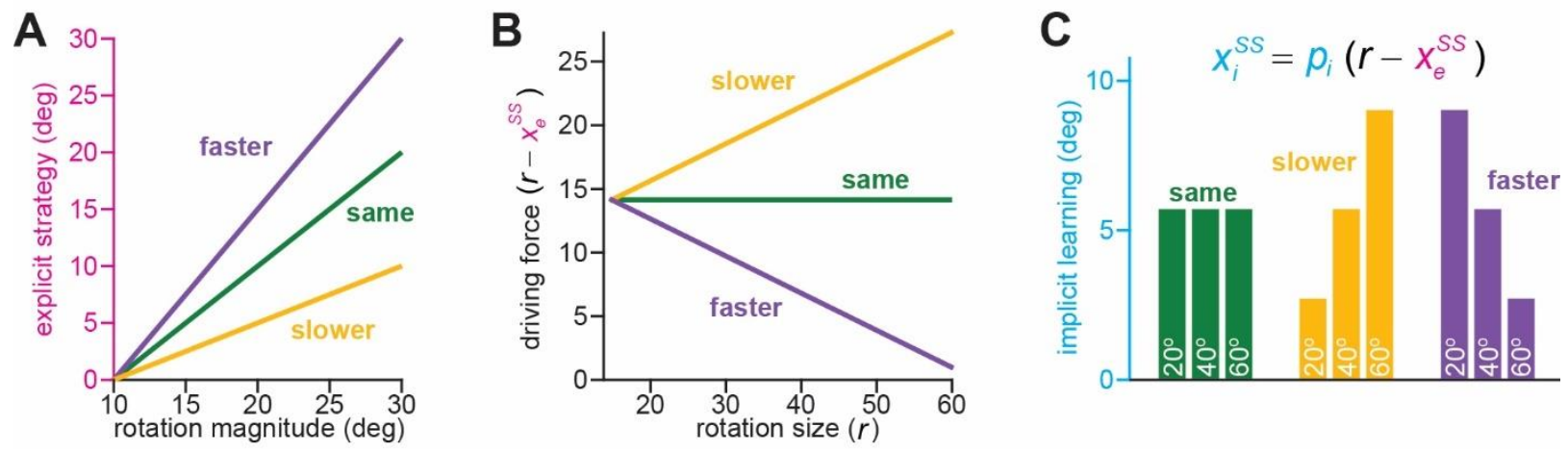

Figure 1-Supplement 1. Implicit learning can exhibit various phenotypes in the competition theory. Here we consider how implicit learning can respond to changes in rotation size in the competition theory. A. Total implicit learning in the competition theory is altered by explicit strategy. We show three cases: (1) strategy increases at the same rate as rotation size ("same", gain=1), (2) strategy increases more slowly than rotation size ("slower", gain<1), (3) strategy increases faster than rotation size ("faster", gain>1). Gain here is equal to each line's slope (it is not dependent on the intercept, which is non-zero). B. In the competition theory, the driving input to the implicit system is the error (i.e., difference) between the rotation and steady-state explicit strategy. Thus, when explicit strategy and rotation size grow by the same amount ("same"), the implicit driving force remains constant. When explicit strategy grows more than the rotation ("faster"), the implicit driving force decreases as the rotation gets larger. When explicit strategy grows less than the rotation ("slower"), the implicit driving force increases as the rotation gets larger. C. In the competition theory (equation at top), implicit learning is proportional to the implicit driving forces depicted in $\mathbf{B}$. The proportionality constant, $p_{i}$, depends on implicit error sensitivity and retention (see Eq. (4)). Thus, in the "same" scenario in $\mathbf{A}$ and $\mathbf{B}$, implicit learning will remain the same across rotation sizes. In the "slower" scenario in A and B, implicit learning will increase with the rotation. In the "faster" scenario in $\mathbf{A}$ and B, implicit learning with decrease as the rotation increases. 


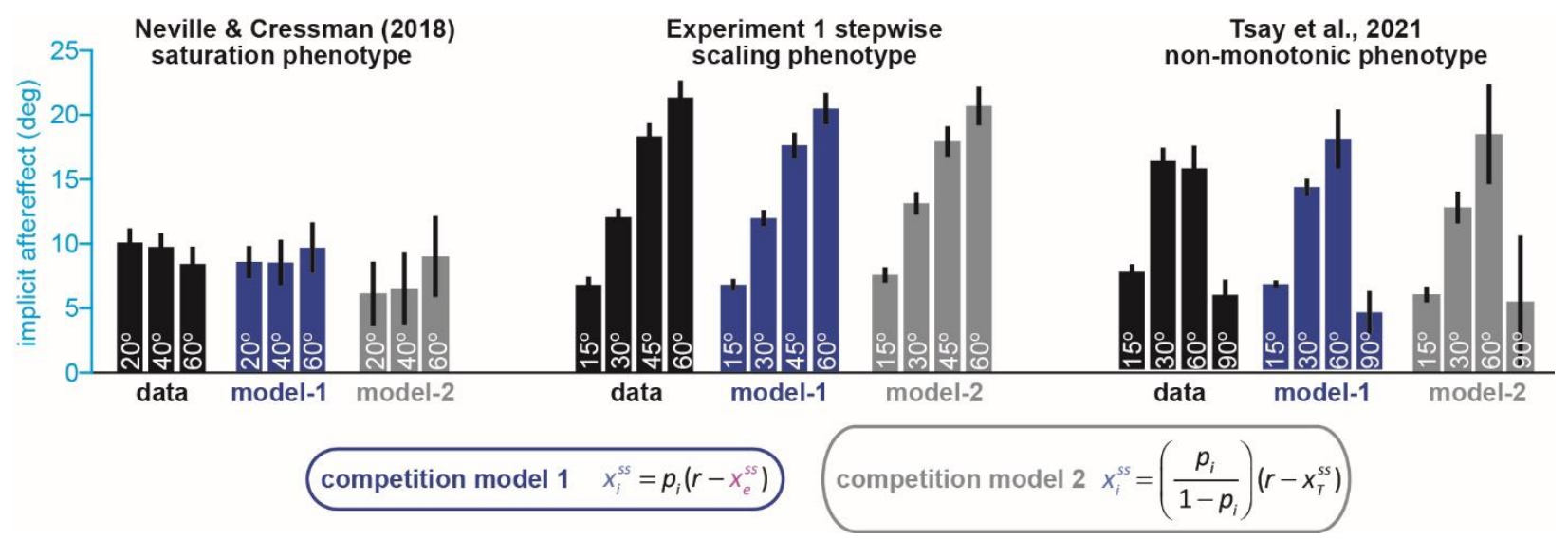

Figure 1-Supplement 2. Variations between total learning and implicit learning are consistent with the competition model. In Fig. 1, we evaluate how well the competition equations matches data across 3 distinct implicit learning phenotypes: saturation, scaling, and non-monotonic responses. These three implicit learning phenotypes are shown again here (data, black bars; each group from left to right shows a different phenotype). The competition model is intuitively stated as a relationship between implicit learning and explicit strategy. This equation is denoted in blue: 'competition model 1'. Blue bars show how much implicit learning was predicted in each experiment, using explicit strategy and 'competition model 1' ('model-1' under each set of bars). The competition model can be stated another way. Noting that total adaptation is equal to the sum of implicit and explicit learning, we can replace explicit learning in 'competition model 1', with total adaptation minus implicit learning. Algebraic simplification yields 'competition model 2', shown in gray. This is an equivalent competition model, only this time, it is stated as a relationship between implicit learning and total adaptation (which were measured on separate trials). The gray bars ('model-2') show how much implicit learning was predicted by 'competition model 2', using measured total adaptation. Competition models 1 (implicit predicted using explicit) and 2 (implicit predicted using total adaptation) yielded nearly identical predictions. More detail on these comparisons is provided in Appendix 1. 


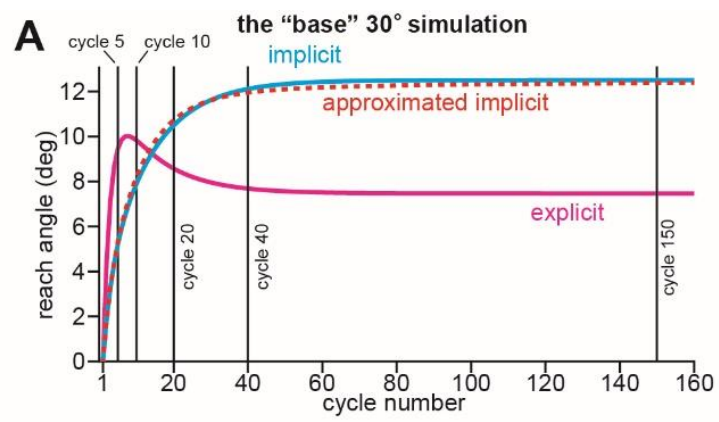

B evolution of the implicit state over time explicit error sensitivity $0.15 \quad 0.15 \quad 0.435 \quad 0.93 \quad$ implicit learning properties are all the same
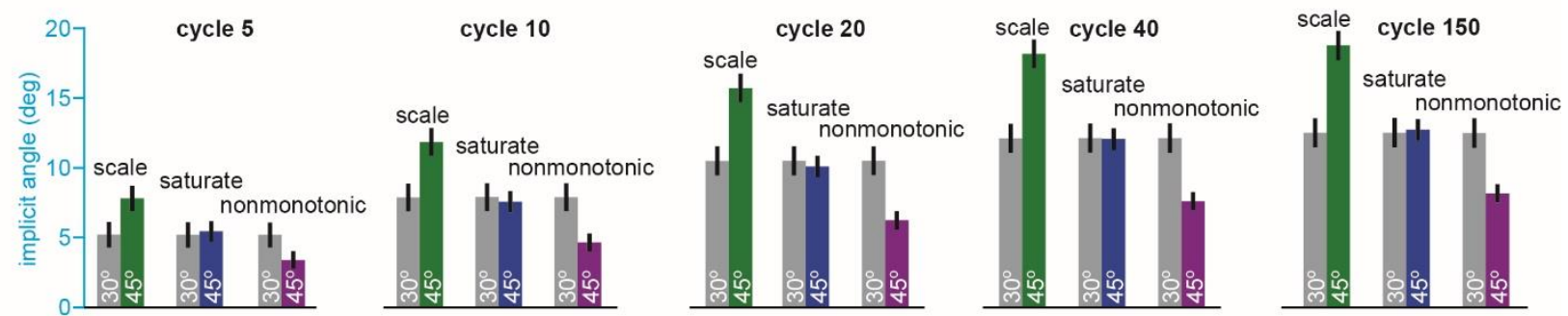

C

evolution of the explicit state over time explicit error sensitivity $\begin{array}{llll}0.15 & 0.15 & 0.435 & 0.93\end{array}$

implicit learning properties are all the same
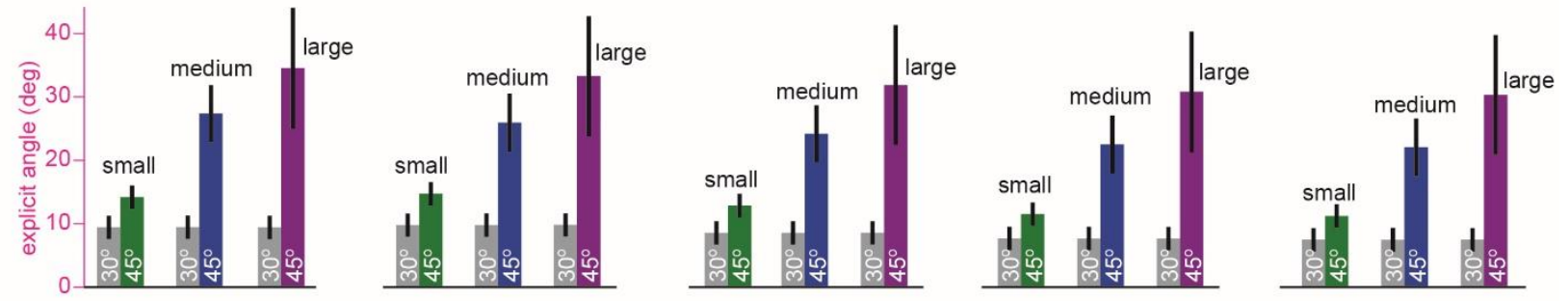

Fig. 1-Supplement 3. Scaling, saturation, and non-monotonic phenotypes across the implicit learning timecourse. A. A "base" simulation where implicit and explicit systems adapt to target error. A response to a $30^{\circ}$ rotation is shown. This response matches the gray bars in $\mathbf{B}$ and $\mathbf{C}$. Note the vertical lines. These indicate moments in time where the implicit and explicit responses were calculated in B and C: from left to right, 5, 10, 20, 40, and 150 rotation cycles. Also note the red dashed "approximated implicit" line. This shows the implicit approximation detailed in Appendix A1.1, where $x_{e}$ is replaced with the average explicit strategy up until that cycle number. In B and $\mathbf{C}$, we show implicit and explicit responses measured at each vertical bar in $\mathbf{A}$. Left to right shows the early-tolate evolution of each adaptive process. B shows implicit learning. C shows explicit learning. Green, blue, and purple bars correspond to a $45^{\circ}$ rotation response. In addition to changing the rotation magnitude, explicit error sensitivity was also modulated to create the scaling, saturation, and nonmonotonic implicit learning modes. In green, $b_{e}$ remained at 0.15 (the same as the gray "base" simulation). In blue, $b_{e}$ was increased to 0.435 . In purple, $b_{e}$ was increased dramatically to 0.93 . The scale, saturate, and nonmonotonic phenotypes can be seen at all timepoints in $\mathbf{B}$. 


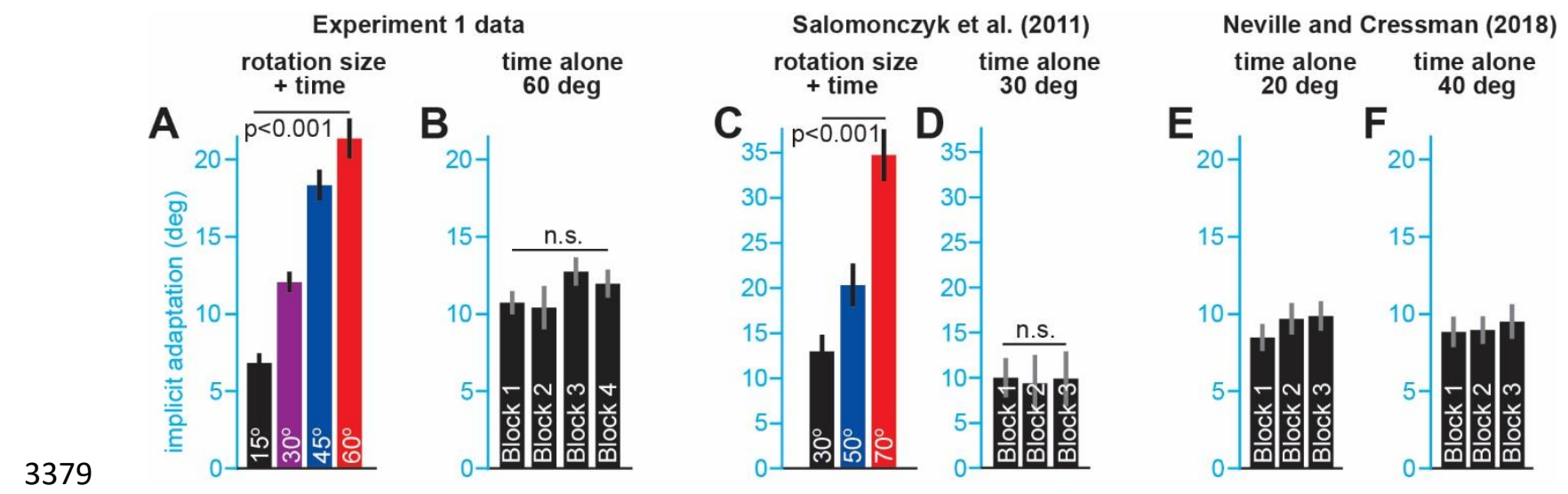

Fig. 1-Supplement 4. Changes in implicit learning across blocks. A. Implicit learning measured during each block in the stepwise group in Exp. 1. B. Implicit learning measured during each block in the abrupt group in Exp. 1. C. Implicit learning measured in a stepwise condition in Salomonczyk et al. (2011) ${ }^{36}$. D. Implicit learning measured in a $30^{\circ}$ group over 3 learning blocks in Salomonczyk et al. (2011). E. Implicit learning measured in a $20^{\circ}$ group over 3 learning blocks in Neville \& Cressman $(2018)^{30}$. F. Same as E, but for a $40^{\circ}$ group. 

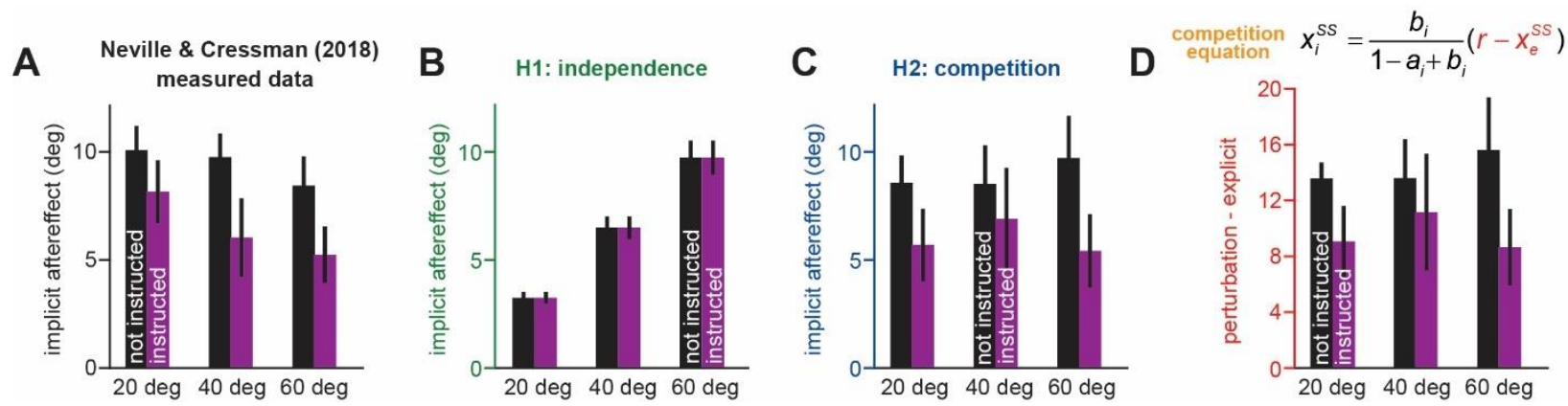

Figure 2-Supplement 1. Changes in implicit adaptation in response to awareness and rotation size. Data reported from Neville and Cressman (2018) ${ }^{30}$. A. Participants were separated into 1 of 6 groups. Groups differed based on verbal instruction (instructed purple; non-instructed black) and rotation magnitude ( $20^{\circ}$ left; $40^{\circ}$ middle; $60^{\circ}$ right). Here we show implicit learning measured using exclusion trials (reach without re-aiming) at the end of adaptation. B. Here we show implicit aftereffects predicted by a model where implicit system learns from SPE only. C. Here we show implicit aftereffects predicted by a model where implicit system learns from target error only. D. The competition theory (target error learning) predicts that implicit learning will be proportional to the difference between the rotation size and the total explicit strategy. Here we show this quantity for all 6 experimental groups. Note that model predictions in $\mathbf{B}$ and $\mathbf{C}$ assume that the implicit learning gain is the same across all $\mathbf{6}$ experimental groups. Error bars for data show mean \pm SEM. Error bars for model predictions refer to mean and standard deviation across 10,000 bootstrapped samples. 


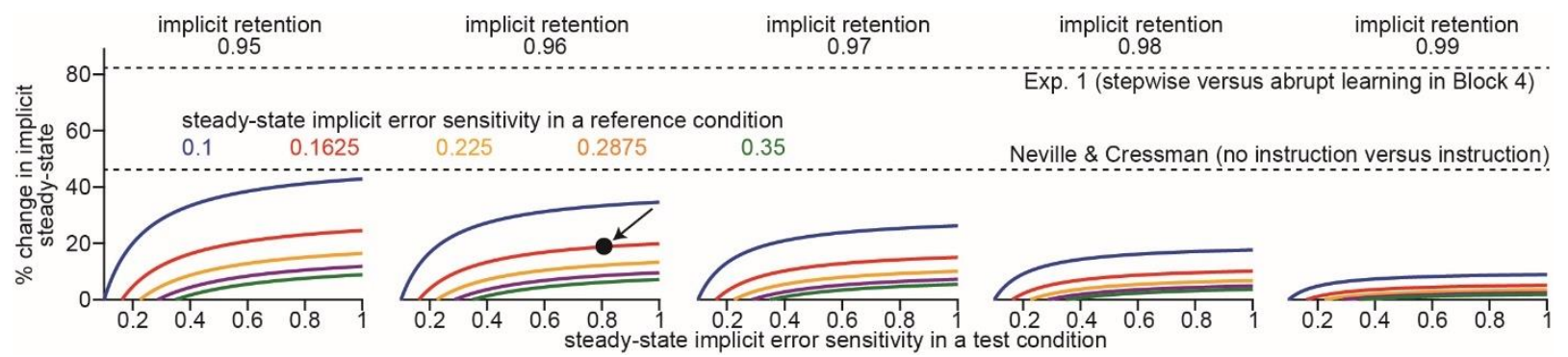

Figure 2-Supplement 2. Total implicit adaptation varies slowly with changes in implicit error sensitivity. We used a sensitivity analysis to explore whether changes in error sensitivity could explain the variations in implicit learning in Exp. 1 (abrupt vs. stepwise) and Neville \& Cressman (instruction vs. no instruction). Above we compare a 'reference' and a 'test' condition. We chose several possible implicit error sensitivity and retention levels in our 'reference'. From left to right, we test implicit retention factors between 0.95 and 0.99 . The colors in each inset, denote different reference error sensitivities: from 0.1 to 0.35 . Each curve shows how much implicit learning will increase in a 'test' condition (i.e., the $y$-axis) over the reference condition; $y$-axis denotes the percent change in total implicit learning and $\mathrm{x}$-axis denotes error sensitivity in the test condition. For example, for the point highlighted by the black arrow in the second column: this point shows that total implicit learning will increase by about $20 \%$ (y-axis) in a scenario where implicit retention $=0.96$, and implicit error sensitivity increases from 0.1625 (i.e., it is on the red line) in the reference condition to 0.8 (i.e., the $x$-axis value) in the test condition. 
3411

3412

3413

3414

3415

3416

3417

3418

3419

3420

3421

3422

3423

3424

3425

3426

3427

3428
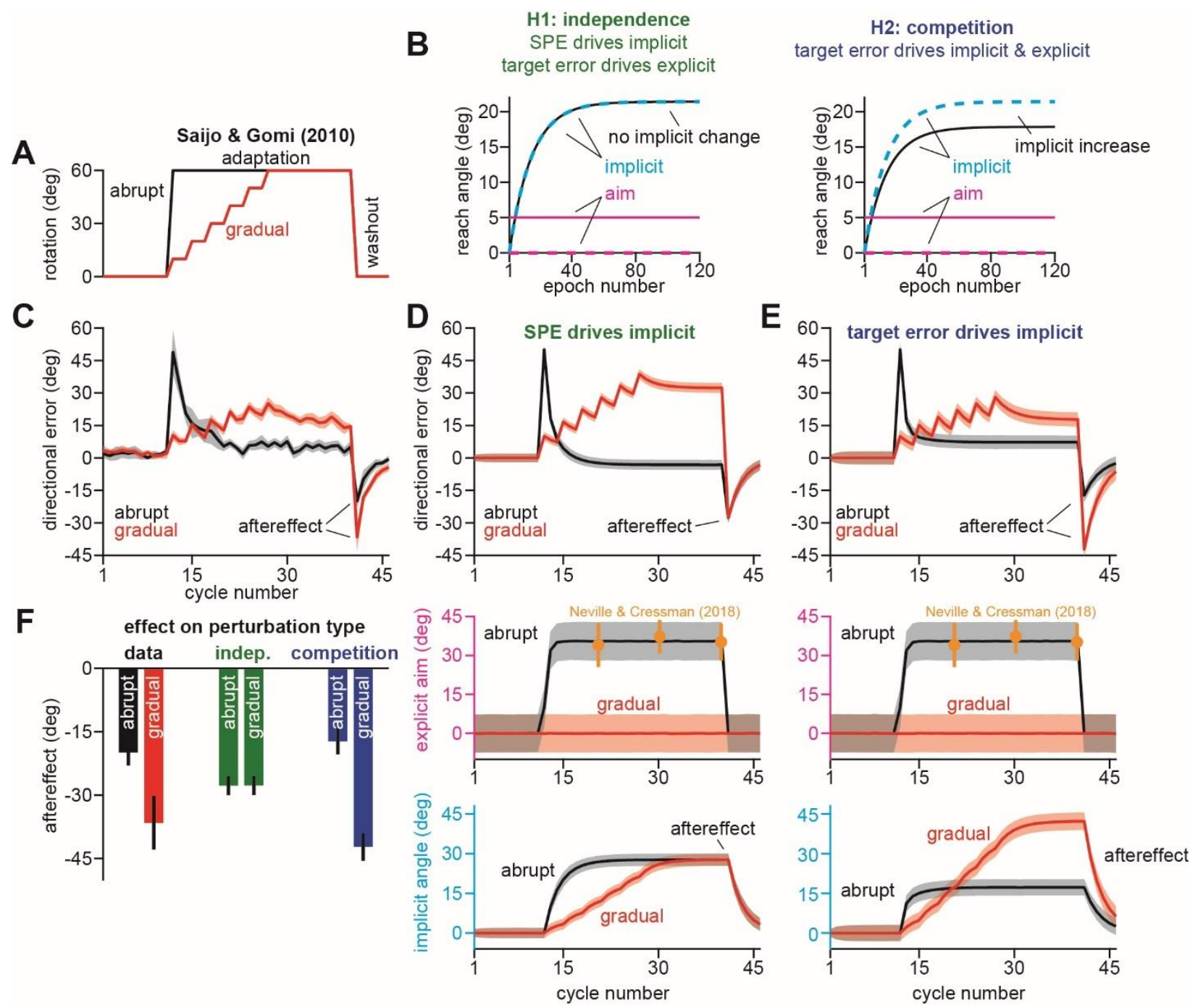

Figure 2-Supplement 3. Suppressing explicit strategy increases total implicit adaptation. Data reported from Saijo and $\mathrm{Gomi}^{33}$. A. Participants experienced an abrupt or gradual $60^{\circ}$ rotation (followed by washout). B. We explored two hypotheses: prediction error ( $\mathrm{H} 1$, aim vs. cursor) vs. target error ( $\mathrm{H} 2$, target vs. cursor) drives implicit learning. Prediction error hypothesis predicts that suppressing aiming (dashed magenta) through gradual perturbation onset will not change implicit learning (black vs. dashed cyan). Target error hypothesis predicts that suppressing aiming (dashed magenta) will increase implicit adaptation (black vs. dashed cyan). C. Directional error during adaptation. Note that while the abrupt group exhibited greater adaptation during the rotation, they also showed a smaller aftereffect suggesting less implicit adaptation. D. We simulated a state-space model where the implicit system learned from SPE. The model parameters were selected to best fit the data in $\mathbf{C}$. In the middle row, hypothetical abrupt explicit strategy was simulated based on data reported by Neville and Cressman ${ }^{30}$ (yellow points). The gradual explicit strategy was assumed to be zero because participants were less aware. At bottom, we show implicit learning predicted by an SPE error source. Note the identical saturation levels. E. Same as in D, but for implicit adaptation based on target error. Note greater implicit learning in gradual condition at the bottom row. Models in $\mathbf{D}$ and $\mathbf{E}$ were fit assuming that implicit error sensitivity and retention are identical across abrupt and gradual conditions. F. Here we show the implicit aftereffect on the first washout cycle (12 total trials). Model predictions for SPE learning (indep.) and target error learning (competition) are shown. Data show mean \pm SEM across participants. Error bars for model are mean and standard deviation across 20,000 bootstrapped samples. 

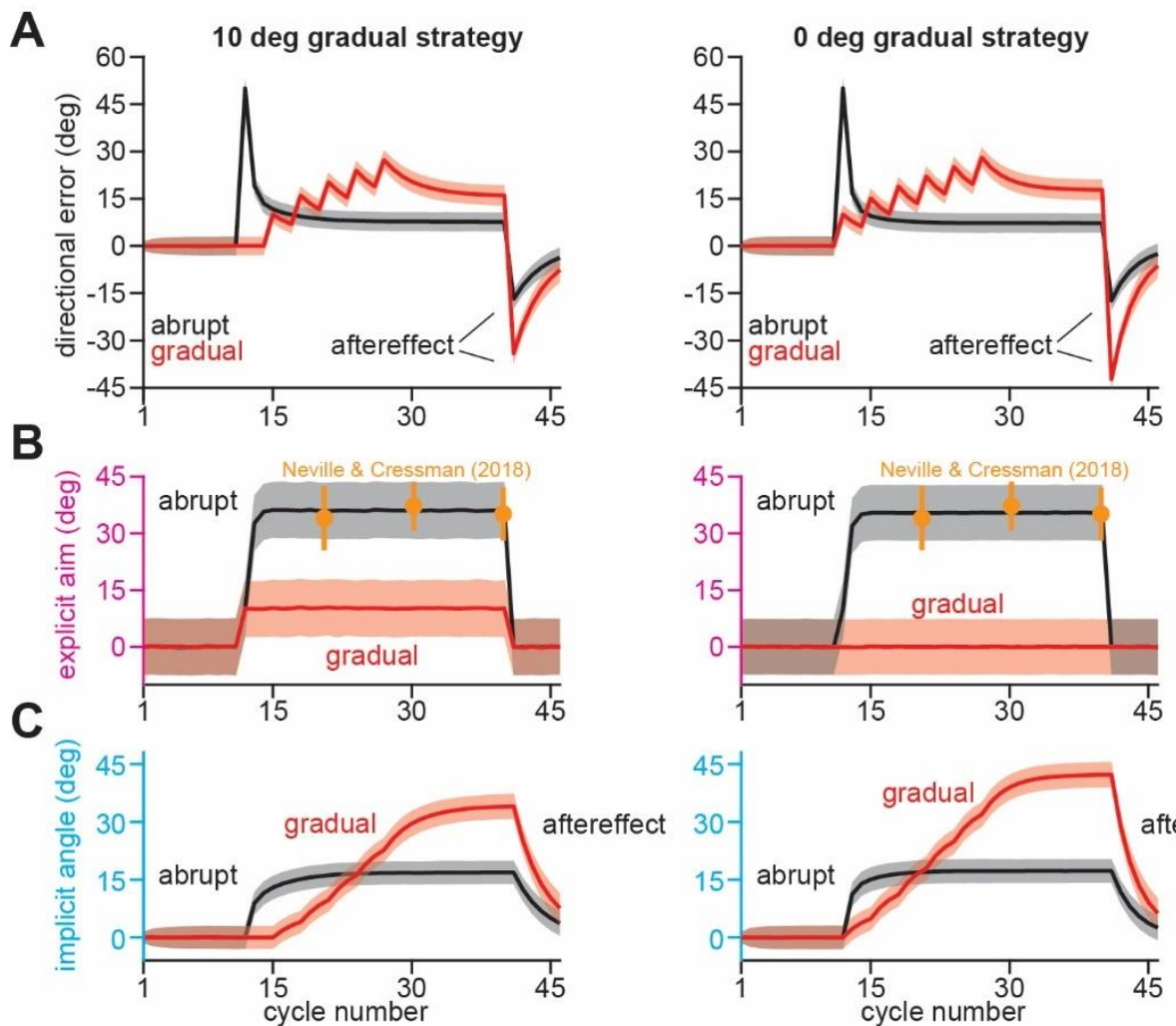

Figure 2-Supplement 4. The competition model is compatible with various explicit strategy levels in Saijo and Gomi (2010). In Appendix 2, we analyzed the Saijo \& Gomi (2010) ${ }^{33}$ abrupt and gradual learning conditions with both the competition and independence models. We measured whether each model could predict total implicit learning,
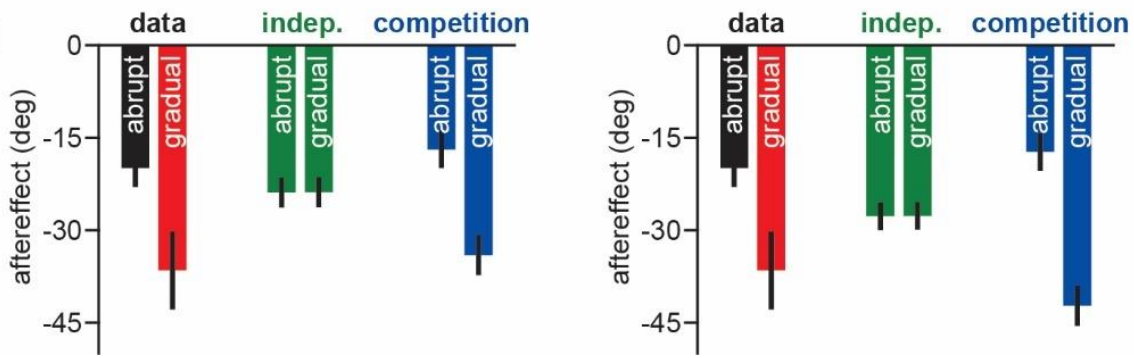
with the assumption that the initial washout reach angle primarily reflected implicit adaptation. These analyses assumed that participants in the gradual condition did not use explicit strategy. In the right column, we reproduce competition model's predictions under this zero strategy assumption, as in Fig. 2-Supplements 2E and F. Next, we repeated these analyses in an alternate scenario, where explicit strategy was assumed to be $10^{\circ}$ during the rotation period. This new control analysis is shown in the left column. We observed that model predictions were qualitatively similar whether gradual learning was simulated with $0^{\circ}$ strategy, or $10^{\circ}$ strategy. A shows directional errors predicted by competition. B shows the explicit strategies used as input in these simulations. C shows the implicit response to the rotation and explicit strategies predicted by the competition model. $\mathbf{D}$ shows the aftereffect predicted by the competition model (blue), independence model (green), and that observed in the actual experiment (data). For the model predictions, we used the implicit angle on the initial washout cycle. 

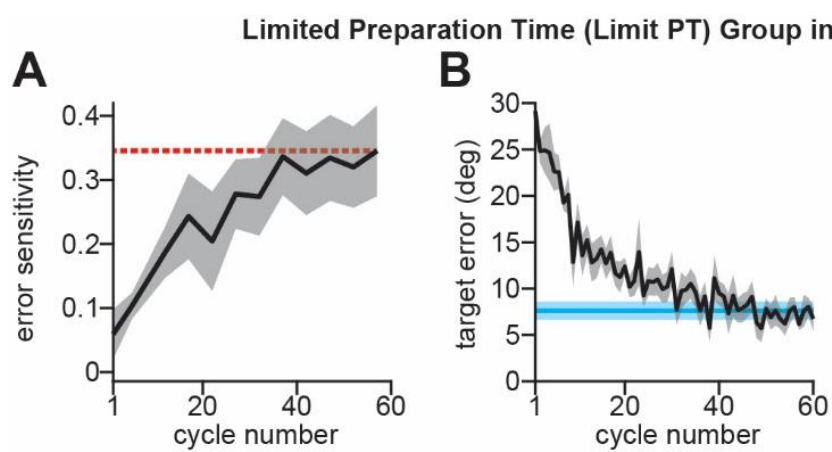

Limited Preparation
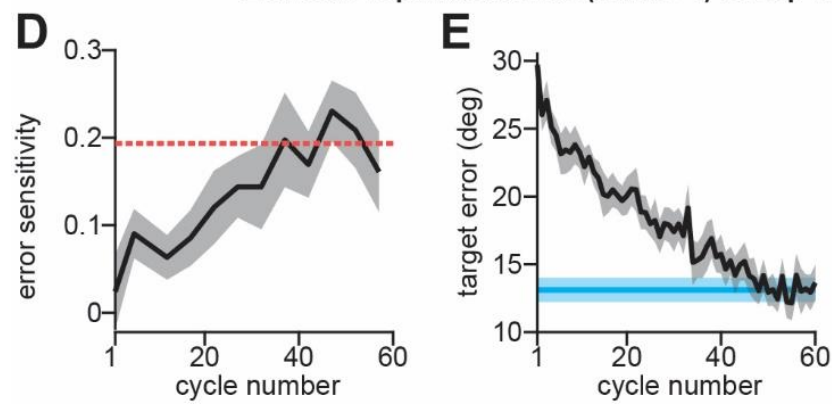

C

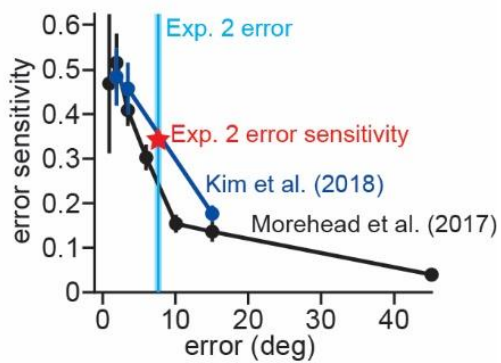

Figure 3-Supplement 1. Implicit error sensitivity varies with error. A. We empirically estimated error sensitivity on each trial in the limited preparation time (Limit PT) group in Experiment 2. The dashed horizontal line indicates the steady-state error sensitivity used in our competition theory predictions in Fig. 3G. B. Here we show error on each trial in the Limit PT group in Experiment 3. The horizontal cyan line shows the terminal error over the last 10 cycles. C. Error sensitivity curves reported in Kim et al. (2018) ${ }^{40}$ denoted E1 (Morehead et al. (2017) ${ }^{1}$ results) and E2 (Kim et al. (2018) results). These two studies used invariant error-clamp tasks to isolate implicit learning. We compared our implicit learning measure in the Limit PT condition in Exp. 2 to these values. The vertical blue line shows the terminal error in $\mathbf{B}$. The red star shows the terminal error sensitivity measured in $\mathbf{A}$. In panels $\mathbf{D}-\mathbf{F}$, we show the same data as in A-C, except for the Limit PT condition in Experiment 3 where participants were tested on a personal laptop. Shaded error bars denote mean \pm SEM across participants. 
A

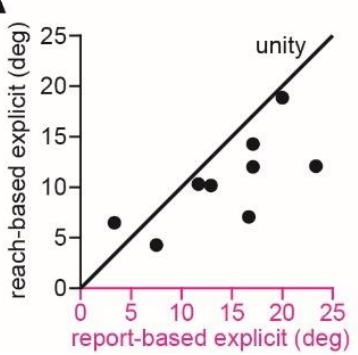

B

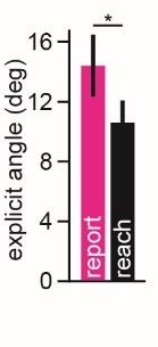

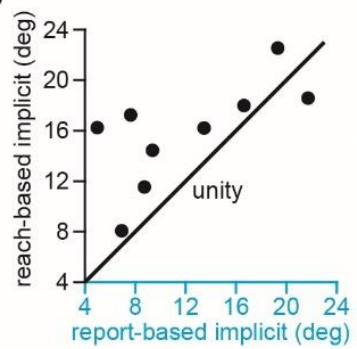

C

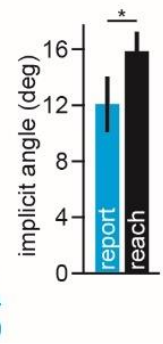

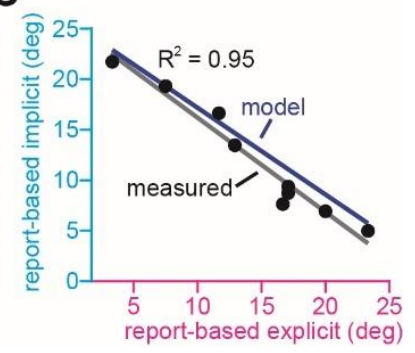

Figure 3-Supplement 2. Comparing implicit and explicit adaptation via reported strategies. In Fig. 3, when analyzing the No PT-Limit group (no preparation time limit) in Experiment 2, we measured implicit learning using exclusion trials at the end of adaptation. Next, we estimated explicit strategies by subtracting this reach-based implicit learning measure from the total adaptation measured over the last 10 cycles of adaptation (reach-based explicit measure). In addition, we also asked participants to report their explicit strategies after the probe period. Participants were shown a ring of circles surrounding each target and asked to indicate which circle best represented their aiming direction at the end of the experiment. We averaged this report-based explicit measure across all 4 adaptation targets, taking the absolute value for any misreported strategies (25\% of all reports in opposite direction). We estimated report-based implicit learning by subtracting the reported explicit strategy from the total adaptation measured over the last 10 rotation cycles. A. Here we compare report-based explicit strategy with reach-based explicit strategy. Each point represents an individual participant. The solid line is the unity line. The bars at right show the mean value for each explicit measure. B. Similar to A except here we compare reportbased implicit learning with reach-based implicit learning. C. Here we compared report-based implicit and reportbased explicit learning measures. We also show the relationship predicted by the competition theory in blue (same as in Fig. 3G). Error bars show mean \pm SEM across participants. Statistics in A and B show paired t-tests: ${ }^{*} p<0.05$. 

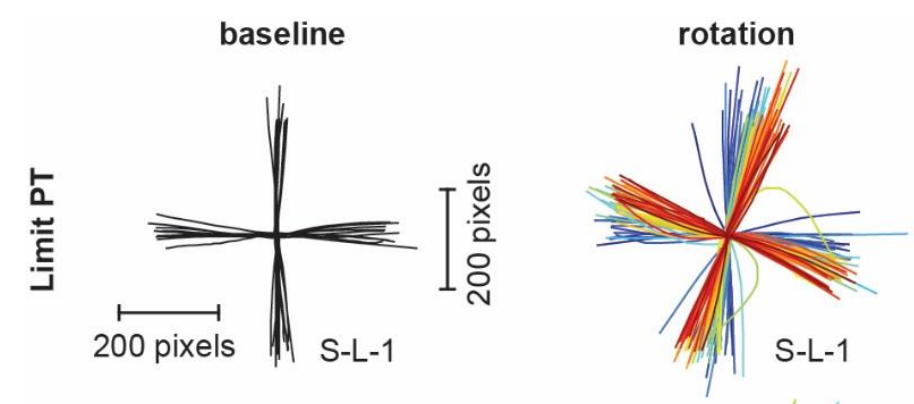

no aiming
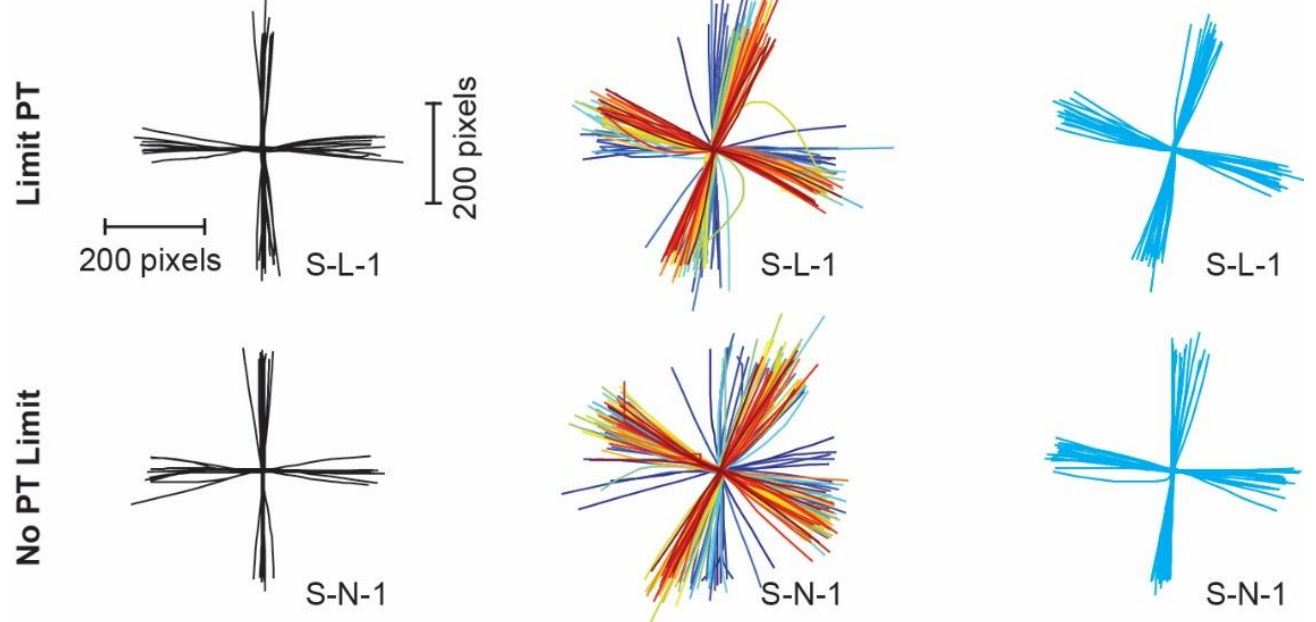

Figure 3-Supplement 3. Movement paths in Exp. 3 were straight and brisk. In Exp. 3, we tested participants remotely in a laptop-based rotation study. Here we show movement paths recorded by the computer in two example subjects: one in the Limit PT group (top row) and one in the No PT Limit group (bottom row). The left column shows trajectories during the baseline period. Note the 4 different groupings reflect the 4 different targets used in the task. The middle column shows trajectories during the rotation period. The color indicates the rotation trial number (blue is early in the rotation period, red is late in the rotation period). The data show a clear rotation in participant movement angle. Reach trajectories remained straight. Finally, the right column shows movement paths during the terminal period where participants were instructed to move straight to each target, without any cursor feedback. 

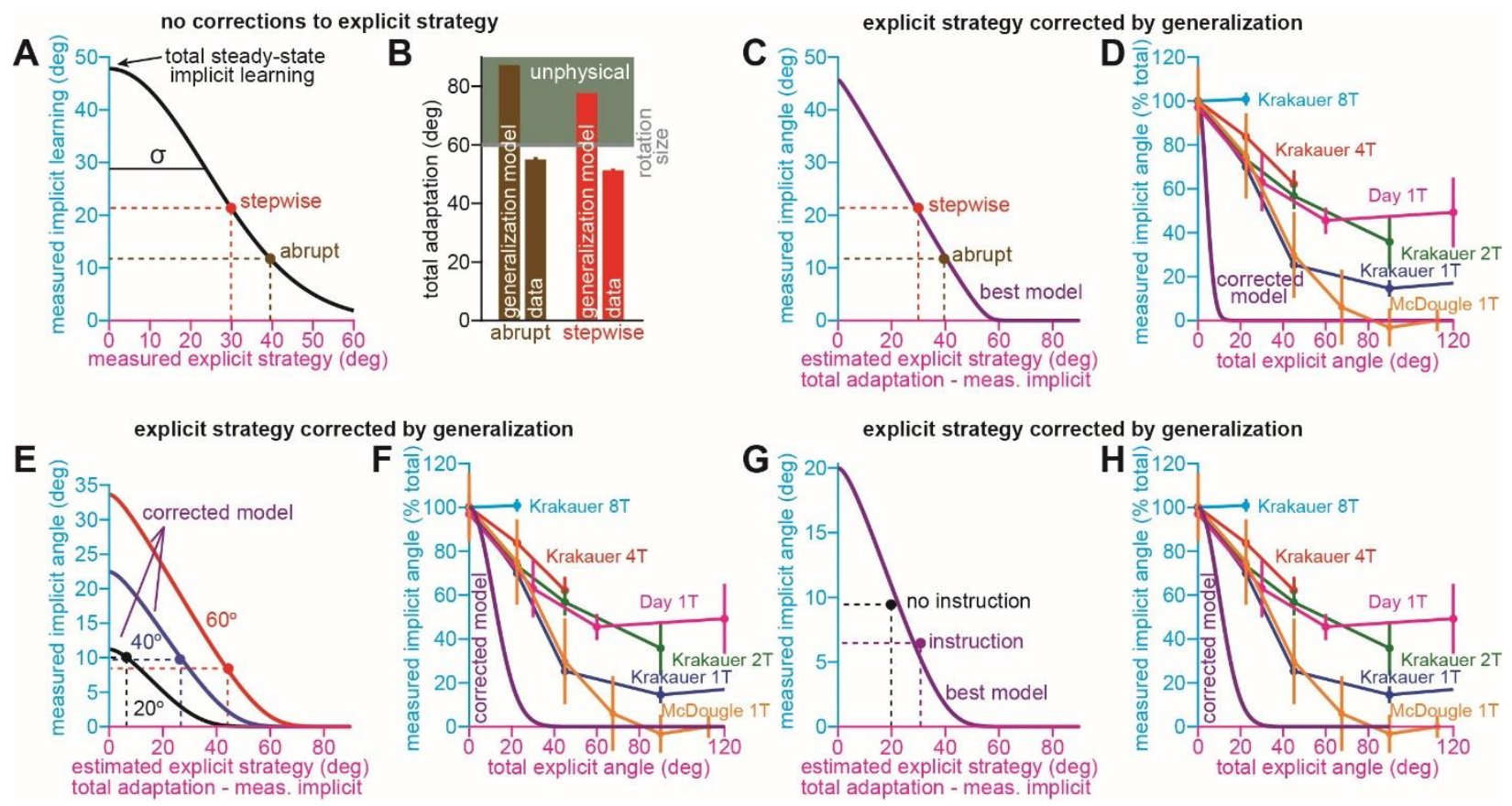

Figure 4-Supplement 1. Generalization is inconsistent with implicit variations. A. We fit a Gaussian generalization curve to the implicit and explicit learning measures in the stepwise (red) and abrupt (brown) groups in Exp. 1. The curve's peak indicates total steady-state implicit learning. For both stepwise and abrupt groups to have the same total implicit learning, this would require total implicit learning to equal $47.8^{\circ}$ (total steady-state implicit learning in the graph) and a standard deviation equal to $23.6^{\circ}$ ( $\sigma$ in the graph). B. The analysis in A does not yield physiological results. Total adaptation would be calculated by adding the total steady-state implicit learning to the explicit strategy ( $x$-axis in A). This would yield total learning equals $87.3^{\circ}$ and $77.7^{\circ}$ in the abrupt and stepwise groups, respectively (bars labeled generalization model). Yet the rotation was only $60^{\circ}$ : gray line (rotation size). Thus, total learning much exceed the rotation to yield the measurements in $\mathbf{A}$. Total learning in both the stepwise and abrupt groups is also provided in the inset (bars labeled data). C\&D. The violation in A\&B is that measured explicit strategy is estimated as total adaptation minus measured implicit learning. Explicit strategy estimate will greatly overestimate true explicit strategy. In these insets, we corrected this in the model. In C we show the model when implicit learning decrements due to generalization, and strategy is estimated as total adaptation minus generalized implicit learning (best model). In $\mathbf{D}$ we show the true underlying generalization curve that produces the implicitexplicit measures in C. The curve is shown in the corrected model. Note that such a curve is physiologically inconsistent with previously measured implicit learning properties. In $\mathbf{E}$ and $\mathbf{F}$, we conduct a similar analysis, but this time analyze the response to the $20^{\circ}, 40^{\circ}$, and $60^{\circ}$ no-instruction rotations in Neville \& Cressman $(2018)^{30}$. E shows the model's behavior when implicit and explicit learning are both biased by generalization. The 3 curves correspond to each rotation size (total implicit learning scales because it is equal to $p_{i}$ ). In $\mathbf{F}$ we show the true Gaussian generalization curve (corrected model) that is needed to produce the data in $\mathbf{C}$. Last, in $\mathbf{G}$ and $\mathbf{H}$, we analyzed the response to instruction in Neville and Cressman. These insets are analogous to $\mathbf{C}$ and $\mathbf{D}$. 
Aftereffects at target and aim direction

A $\begin{gathered}\text { Day et } \\ \text { al. (2016) }\end{gathered} \quad \begin{gathered}\text { McDougle et } \\ \text { al. (2017) }\end{gathered}$

all report trials
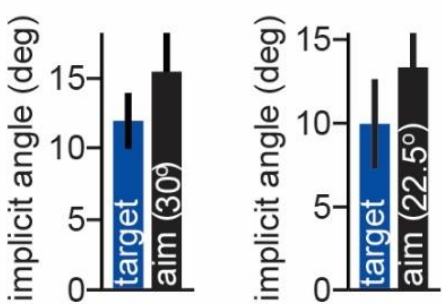

Comparing aim reports to implicit learning measured at target

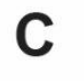

Day et

al. (2016)

D Maresch et al. (2020)

E Experiment 2

all report trials

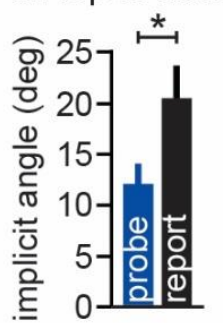

$5 \%$ report trials

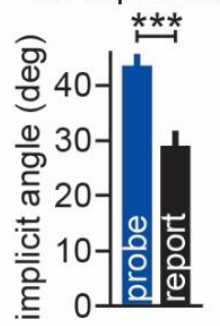

no report trials

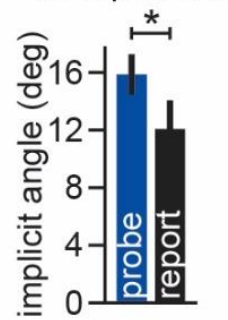

3507

3509

3510

3511

3512

3513

3514

3515

3516

3517

3518

3519

3520

3521

3522

3523

3524

3525

3526

3527

3528

3529

3530

Figure 4-Supplement 2. Differences in generalization across visuomotor rotation tasks. A. Data collected by Day et al. $^{49}$, reported in Fig. 2 of the original manuscript (Fig. 4 in current manuscript). Here, participants were exposed to a $45^{\circ}$ rotation while reaching to a single target. On each trial they were asked to report their aiming direction, using a ring of visual landmarks. In the "target" group, implicit aftereffects were measured at the trained target location. In the "aim" group, implicit aftereffects were probed at a target location $30^{\circ}$ away from the trained target, consistent with the direction of the most frequently reported aim. Here we show data from the first aftereffect cycle after the rotation period. B. Similar to A except for data reported by McDougle et al ${ }^{50}$ (Fig. $3 \mathrm{~A}$ of the original manuscript). Participants were also exposed to a $45^{\circ}$ rotation while reaching to a single target. At the end of the experiment, participants were exposed to an aftereffect block where participants were told to move straight to the target without re-aiming. Here we take two relevant points from the generalization curve measured at the end of learning. The "target" condition represents aftereffects probed at the training target. The "aim" condition shows the aftereffect measured at $22.5^{\circ}$ away from the primary target, which was the target closest to the mean reported explicit re-aiming strategy of $26.2^{\circ}$. C. Data again from Day et al. The "probe" implicit learning measure is the same as A. The "report" condition shows the amount of implicit learning estimated by subtracting the reported explicit strategy from the reported reach angle on the last cycle of the rotation. D. Similar to $\mathbf{C}$, but for the intermittent reporting (IR-E) group reported by Maresch et al. (Fig. $4 \mathrm{~b}$ of the original manuscript). In this group aim was only intermittently reported (4 trials for every 80 normal adaptation trials). Thus, in most cases, participants only had to attend to a single target when reaching. The authors also used 8 training targets (as opposed to 1 in A-C). The "probe" condition corresponds to the total implicit learning measured at the end of adaptation by telling participants to reach without re-aiming. The "report" condition corresponds to the total implicit learning estimated at the end of adaptation by subtracting the reported aim direction from the measured reach angle. E. Here we report implicit learning measured using the "probe" and "report" conditions in Experiment 2, analogous to the measures described in D. Error bars show mean \pm 
A

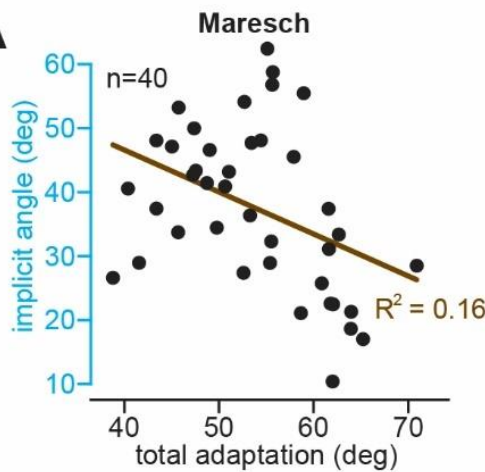

D
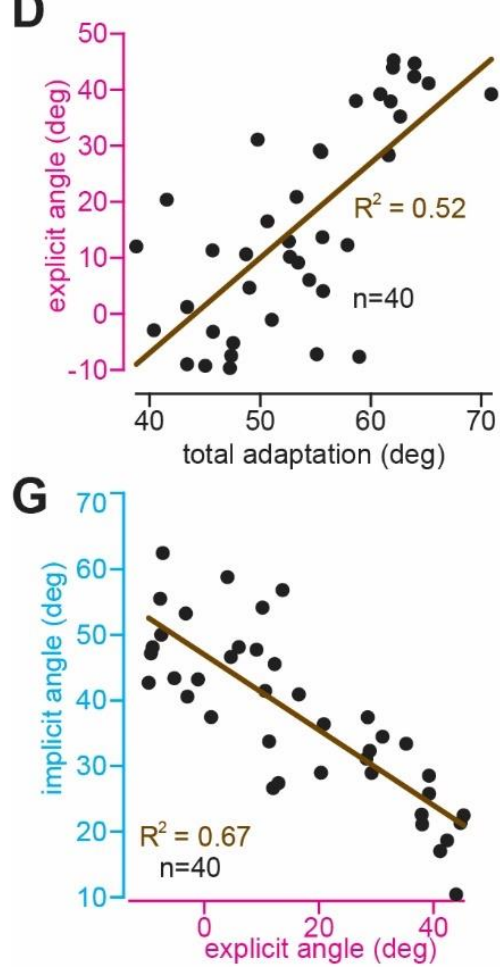

B

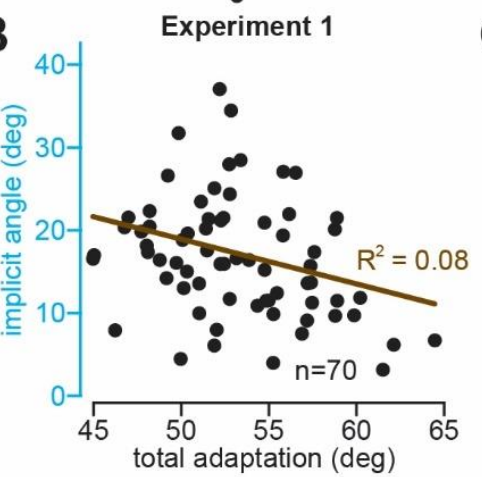

E

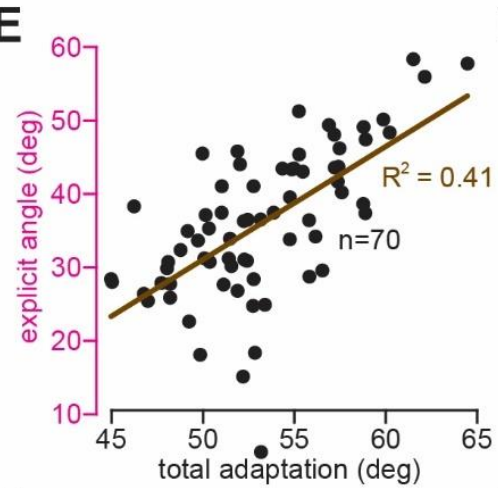

H

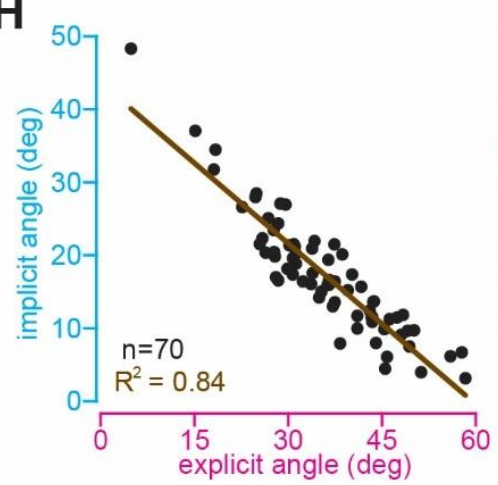

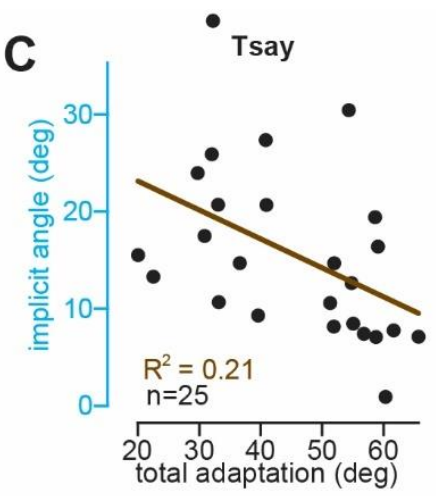

F
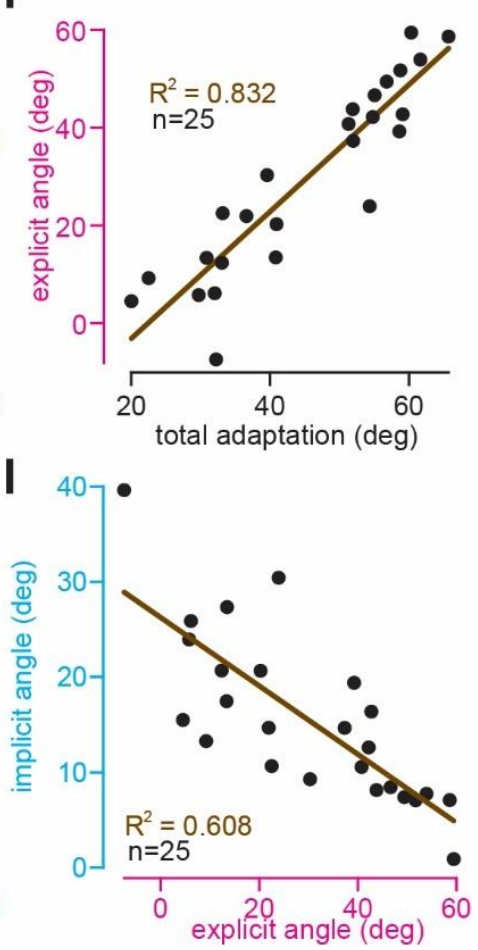

Figure 5-Supplement 1. Relationships between implicit, explicit, and total learning indicate competition. Data were analyzed across 3 experiments. In the left column, we report participants in the CR, IR-E, and IR-EI groups in Maresch et al. ${ }^{41}$ In the middle column, we report participants collapsed across the abrupt and stepwise $60^{\circ}$ rotation period in Experiment 1 . In the right column, we report participants in the $60^{\circ}$ rotation group in Tsay et al. ${ }^{35}$ In each experiment, we analyzed implicit learning, explicit learning, and total adaptation. Implicit and explicit learning were estimated with exclusion ("no aiming") trials. A-C. The relationship between implicit learning and total adaptation. D-F. The relationship between explicit learning and total adaptation. G-I. Relationship between implicit and explicit learning. All lines in A-I denote a linear regression. The associated $\mathrm{R}^{2}$ statistic is shown in each inset. All relationships were statistically significant $(p<0.05)$. Dots in each inset denote individual participants. 

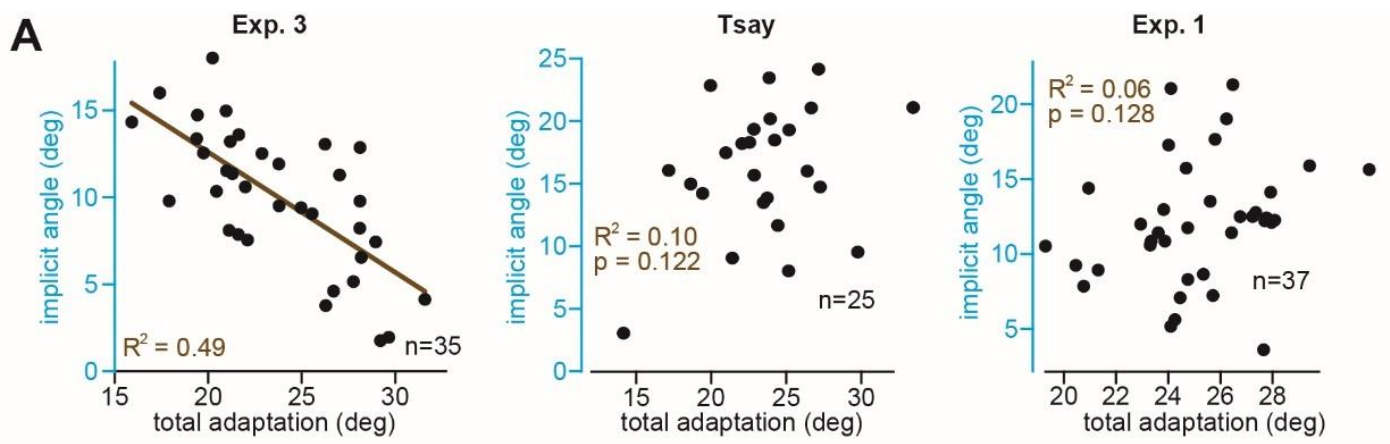

B more implicit aftereffect trials increases probability
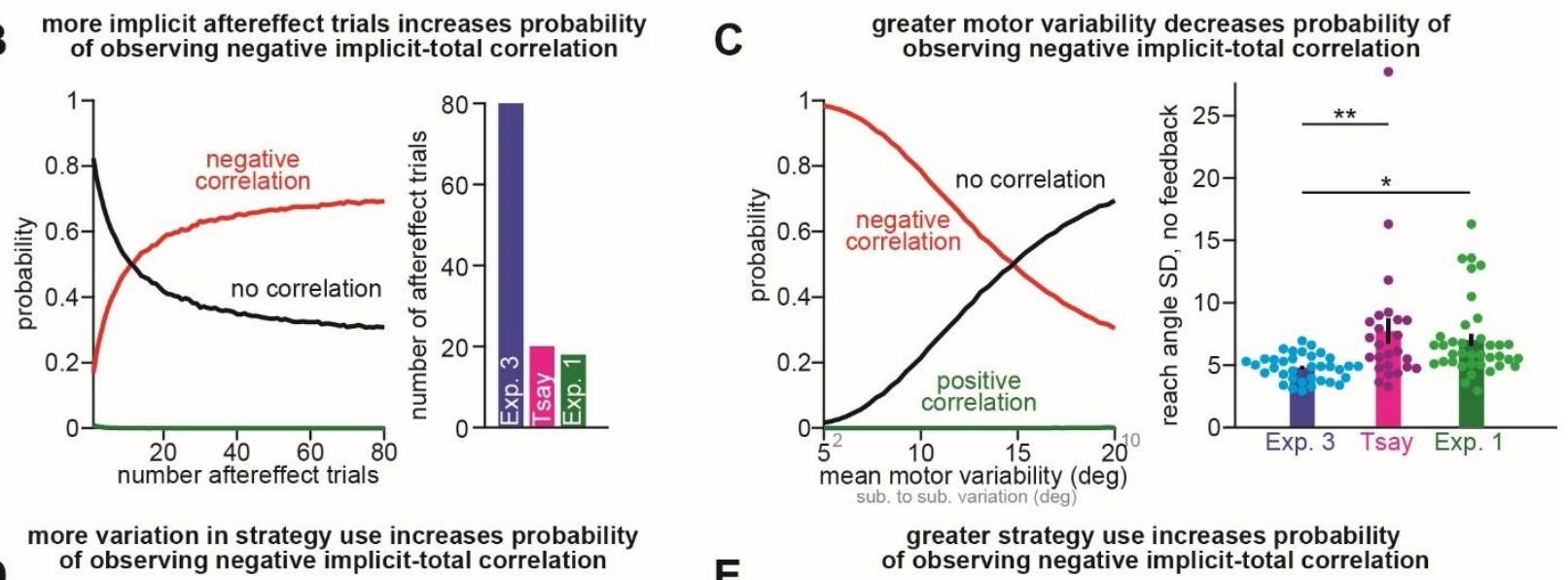

more variation in strategy use increases probability

D

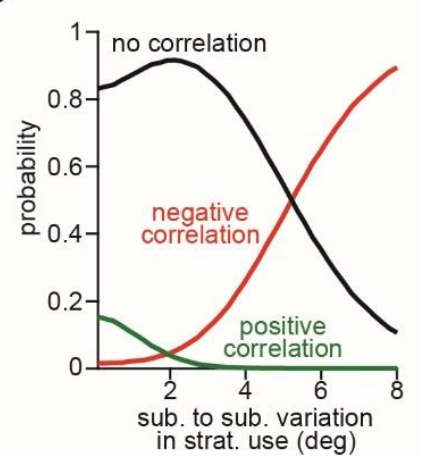

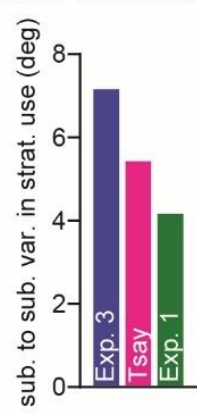

3543

Figure 5-Supplement 2. Factors that weaken the correlation between implicit learning and total adaptation. A. At left we reproduce the relationship between implicit learning and total adaptation in the No PT Limit group in Experiment 3. In the middle inset, the same analysis is shown for participants in the $30^{\circ}$ group in Tsay et al. ${ }^{35}$ At right, the same analysis is shown for participants in the stepwise $30^{\circ}$ rotation period in Experiment 1 . Relationships between implicit learning and total adaptation were not statistically significant $(p>0.05)$ at middle and right. In B-E we explore factors that can weaken the relationship between implicit learning and total adaptation in the competition theory. The four factors are: B, total number of aftereffect trials used to measure implicit learning, $\mathbf{C}$, motor variability in the reach, $\mathbf{D}$, between-subject variability in strategy use, and $\mathbf{E}$, total strategy use in the subject population. At left in each inset we conducted a power analysis. In this power analysis, $n=30$ participants were simulated. Explicit strategies were randomly sampled. Implicit learning was then obtained via the competition equation. Implicit, explicit, and total learning were calculated for each simulated participant, by averaging over a set number of trials. Simulations were repeated 40,000 times. The probability that a negative relationship (red line), positive relationship (green line), and no relationship (black line) occurred is shown in the left inset. In B, at 
3558 left, we show that with fewer trials to measure implicit learning, the probability that an experiment will yield a 3559 statistically significant relationship between implicit learning and total adaptation decreases substantially. At right, 3560 we compare the total number of "no aiming" trials used to measure implicit learning in Exp. 3, Tsay et al., and Exp. 35611 (stepwise). In C, at left, we show that increases in trial-to-trial reach variability (i.e., motor execution noise) 3562 dramatically reduce the probability than an experiment will produce a statistically significant relationship between 3563 implicit learning and total adaptation. At right, we analyze trial-to-trial variability during the no aiming period in 3564 each experiment. In $\mathbf{D}$, at left, we show that little variability in strategy use across participants reduces the 3565 probability that an experiment will yield a negative relationship between implicit learning and total adaptation. At 3566 right, we show the standard deviation in explicit strategies across subjects in the 3 experiments. In $\mathbf{E}$, at left, we 3567 show that little overall strategy use in the subject population decreases the probability that an experiment will 3568 yield a negative relationship between implicit learning and total adaptation. At right, we compare explicit 3569 strategies across the 3 experiments. Statistics in C and $\mathbf{E}$ denote a one-way ANOVA. 

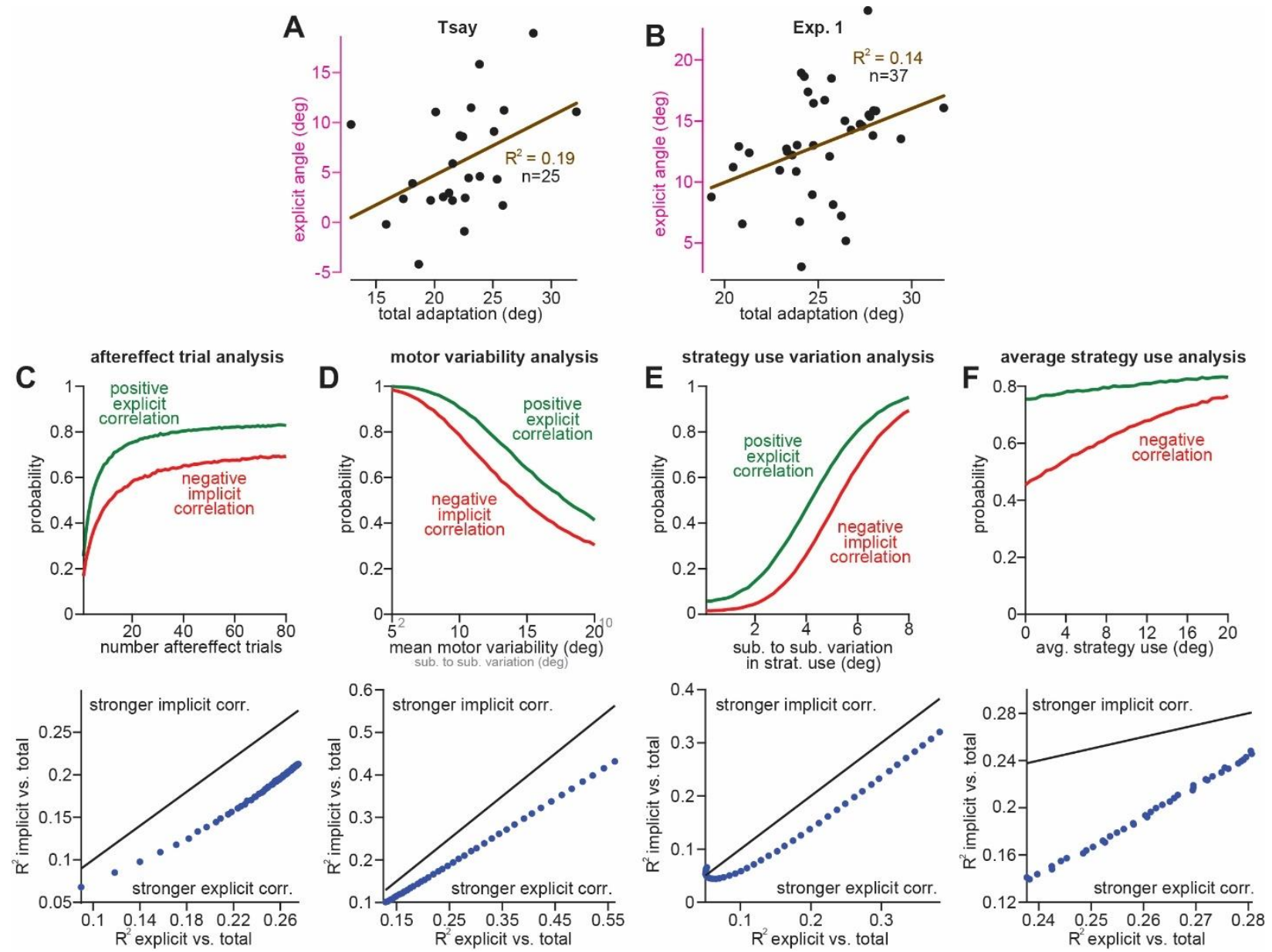

Figure 5-Supplement 3. Correlations between explicit learning and total adaptation are more robust to betweensubject implicit variability. A. Here we show the correlation between explicit strategy and total adaptation in the $30^{\circ}$ rotation group in Tsay et al. ${ }^{35} \mathbf{B}$. Same as A, but for the stepwise $30^{\circ}$ rotation period in Experiment 1 . In C-F we show implicit and explicit correlations with total adaptation can be weakened by 4 factors: $\mathbf{C}$, total number of aftereffect trials used to measure implicit and explicit learning, D, motor variability in the reach, E, betweensubject variability in strategy use, and $\mathbf{F}$, total strategy use in the subject population. At left in each inset we conducted a power analysis. In this power analysis, $n=30$ participants were simulated. Explicit strategies were randomly sampled. Implicit learning was then obtained via the competition equation. Implicit, explicit, and total learning were calculated for each simulated participant, by averaging over a set number of trials. Simulations were repeated 40,000 times. At top we show the probability over these iterations that a statistically significant positive relationship between explicit strategy and total adaptation (green lines) and negative relationship between implicit learning and total adaptation (red lines) occur. At bottom, we calculated the average $R^{2}$ value for the implicit-total and explicit-total regressions. Each point compares the two $\mathrm{R}^{2}$ values for each simulation condition above, with the unity line (black). In C, we show that more aftereffect trials improves the probability of obtaining statistically significant correlations, but the explicit-total correlation is stronger than the implicit-total correlation. In D, we show the less motor variability improves the probability of obtaining statistically significant correlations, but the explicit-total correlation is stronger than the implicit-total correlation. In E, we show that greater subject-tosubject variability in strategy improves the probability of obtaining statistically significant correlations, but the explicit-total correlation is stronger than the implicit-total correlation. In F, we show that greater overall strategy 
3592 improves the probability of obtaining statistically significant correlations, but the explicit-total correlation is 3593 stronger than implicit-total correlation. 

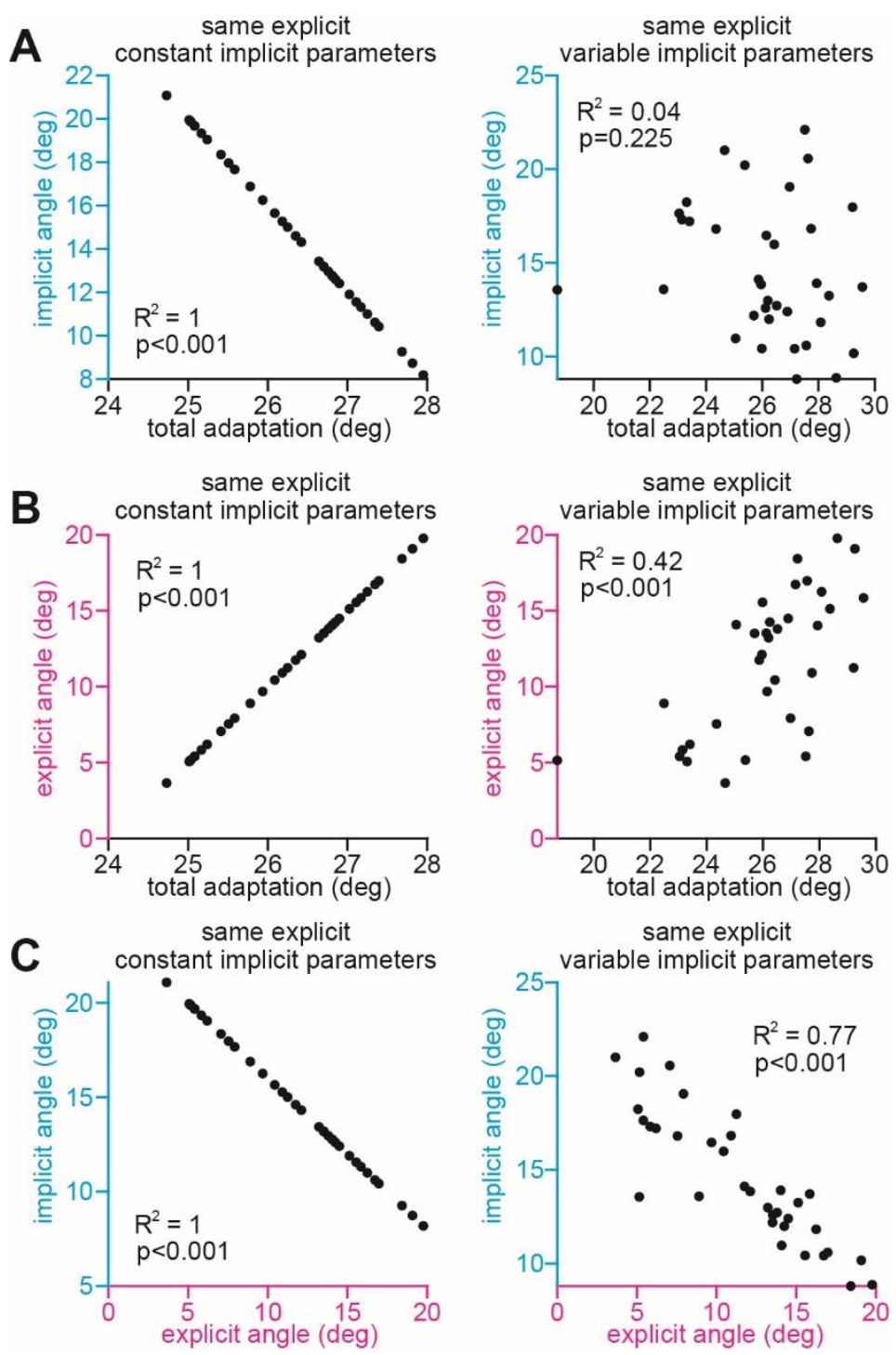

Figure 5-Supplement 4. Variance in implicit learning properties weakens the relationship between implicit learning and total adaptation in the competition theory. Here we consider two sources of variability: (1) subject-to-subject variability in explicit strategy, and (2) subject-to-subject variability in implicit learning. We simulate explicit strategies across 35 participants. The explicit strategies are the same in the left column (same explicit) and the right column (same explicit). They are sampled from a normal distribution: mean $=12^{\circ}, \mathrm{SD}=4^{\circ}$. However, the left and right columns differ in terms of implicit variability. In the left column, there is no implicit variability across subjects. Implicit learning was calculated using the competition theory, using the same implicit learning gain $\left(p_{i}=\right.$ 0.8). In the right column, we added variability to this implicit learning gain. This represents the more realistic scenario, that implicit retention and error sensitivity vary across participants. The implicit learning gain was randomly sampled with a normal distribution, mean $=0.8, S D=0.1$. In $\mathbf{A}$ we show the relationship between implicit learning and total adaptation in these toy cases. In B, we show the relationship between explicit learning and total adaptation in these toy cases. In $\mathbf{C}$, we show the relationship between implicit learning and explicit learning in these toy cases. Note that the relationship between implicit learning and total adaptation ( $\mathbf{A}$, at right) was uniquely susceptible to variability $(p=0.225)$, whereas other correlations remained strong and statistically significant ( $B$ and 


\section{A}
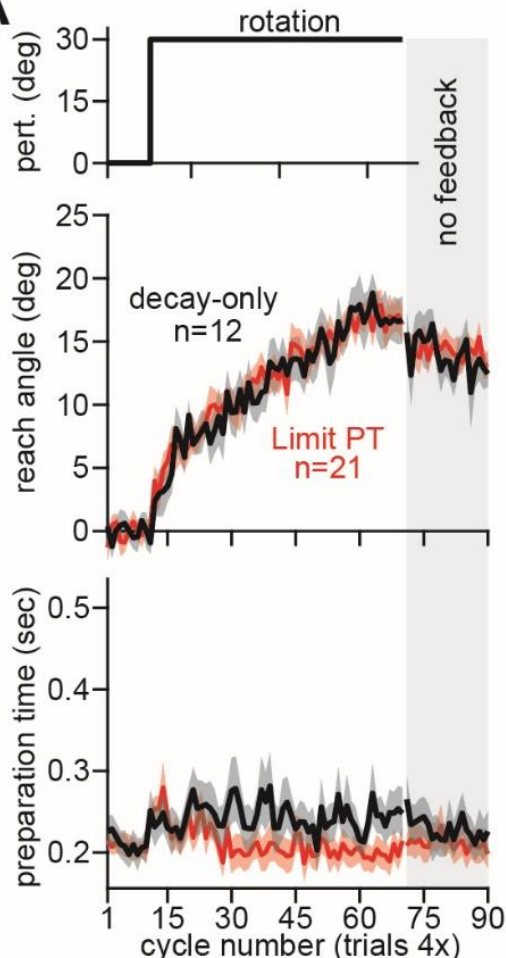

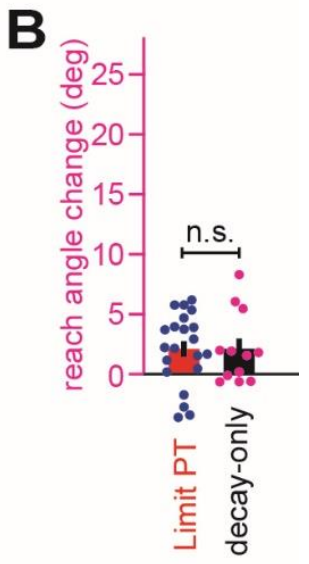

Figure 8-Supplement 1. Limiting preparation time eliminates explicit strategy use. A. Participants were exposed to a $30^{\circ}$ rotation. The perturbation schedule is shown at top. The reach angle on each cycle is shown in the middle. The preparation time on each cycle is shown at bottom. On each trial we imposed a strict upper limit on reaction time in an attempt to suppress explicit strategy. Participants were separated into two groups. These groups had an identical perturbation schedule but received different instructions prior to the terminal no feedback period. In the Limit PT group ( $n=21$, red), participants were instructed to aim directly at the target without re-aiming. In the decay-only group ( $n=12$, black), participants were instructed to imagine the rotation was still present, and to continue trying to move the "imagined cursor" through the target. Change in reach angle in the Limit PT no feedback condition could be due to abandoning explicit strategy and also decay in a temporally-labile implicit process (instruction period was about 30 seconds in duration). Change in reach angle in the decay-only no feedback condition was intended to isolate any decay in a temporally-labile implicit process. B. Change in reach angle due to instruction. Here we subtracted the initial reach angle during the no feedback period from the total adaptation measured prior to instruction. We observed no statistically significant difference between the two groups (two-sample t-test) suggesting that change in reach angle during the no feedback period was almost entirely due to involuntary decay in a temporally-labile implicit process. Error bars indicate mean \pm SEM. 


\section{References}

3629

3630

3631

3632

3633

3634

3635

3636

3637

3638

3639

3640

3641

3642

3643

3644

3645

3646

3647

3648

3649

3650

3651

3652

3653

3654

3655

3656

3657

3658

1. Morehead, J. R., Taylor, J. A., Parvin, D. E. \& Ivry, R. B. Characteristics of Implicit Sensorimotor Adaptation Revealed by Task-irrelevant Clamped Feedback. Journal of Cognitive Neuroscience 29, 1061-1074 (2017).

2. Mazzoni, P. \& Krakauer, J. W. An implicit plan overrides an explicit strategy during visuomotor adaptation. Journal of Neuroscience 26, 3642-3645 (2006).

3. Leow, L.-A., Marinovic, W., de Rugy, A. \& Carroll, T. J. Task errors drive memories that improve sensorimotor adaptation. Journal of Neuroscience (2020) doi:10.1523/JNEUROSCI.1506-19.2020.

4. Körding, K. P. \& Wolpert, D. M. The loss function of sensorimotor learning. Proceedings of the National Academy of Sciences 101, 9839 LP - 9842 (2004).

5. Langsdorf, L., Maresch, J., Hegele, M., McDougle, S. D. \& Schween, R. Prolonged response time helps eliminate residual errors in visuomotor adaptation. Psychonomic Bulletin \& Review 28, 834-844 (2021).

6. Tseng, Y.-W., Diedrichsen, J., Krakauer, J. W., Shadmehr, R. \& Bastian, A. J. Sensory prediction errors drive cerebellum-dependent adaptation of reaching. Journal of Neurophysiology 98, 54-62 (2007).

7. Kawato, M. Internal models for motor control and trajectory planning. Current Opinion in Neurobiology 9, 718-727 (1999).

8. Taylor, J. A. \& Ivry, R. B. Flexible cognitive strategies during motor learning. PLOS Computational Biology 7, e1001096 (2011).

9. Wong, A. L. \& Shelhamer, M. Using prediction errors to drive saccade adaptation: the implicit double-step task. Experimental Brain Research 222, 55-64 (2012).

10. Kim, H. E., Parvin, D. E. \& Ivry, R. B. The influence of task outcome on implicit motor learning. elife 8, e39882 (2019).

11. McDougle, S. D., Bond, K. M. \& Taylor, J. A. Explicit and Implicit Processes Constitute the Fast and Slow Processes of Sensorimotor Learning. Journal of Neuroscience 35, 9568-9579 (2015).

12. Miyamoto, Y. R., Wang, S. \& Smith, M. A. Implicit adaptation compensates for erratic explicit strategy in human motor learning. Nature Neuroscience 23, 443-455 (2020).

13. Haith, A. M., Huberdeau, D. M. \& Krakauer, J. W. The Influence of Movement Preparation Time on the Expression of Visuomotor Learning and Savings. Journal of Neuroscience 35, 5109-5117 (2015). 
14. Coltman, S. K., Cashaback, J. G. A. \& Gribble, P. L. Both fast and slow learning processes contribute to savings following sensorimotor adaptation. Journal of Neurophysiology 121, 15751583 (2019).

15. Kojima, Y., Iwamoto, Y. \& Yoshida, K. Memory of Learning Facilitates Saccadic Adaptation in the Monkey. Journal of Neuroscience 24, 7531-7539 (2004).

16. Medina, J. F., Garcia, K. S. \& Mauk, M. D. A mechanism for savings in the cerebellum. Journal of Neuroscience 21, 4081-4089 (2001).

17. Mawase, F., Shmuelof, L., Bar-Haim, S. \& Karniel, A. Savings in locomotor adaptation explained by changes in learning parameters following initial adaptation. Journal of Neurophysiology 111, 1444-1454 (2014).

18. Sing, G. C. \& Smith, M. A. Reduction in learning rates associated with anterograde interference results from interactions between different timescales in motor adaptation. PLOS Computational Biology 6, e1000893 (2010).

19. Lerner, G. et al. The Origins of Anterograde Interference in Visuomotor Adaptation. Cerebral Cortex 30, 4000-4010 (2020).

20. Caithness, G. et al. Failure to consolidate the consolidation theory of learning for sensorimotor adaptation tasks. Journal of Neuroscience 24, 8662-8671 (2004).

21. Huberdeau, D. M., Krakauer, J. W. \& Haith, A. M. Practice induces a qualitative change in the memory representation for visuomotor learning. Journal of Neurophysiology 122, 1050-1059 (2019).

22. Morehead, J. R., Qasim, S. E., Crossley, M. J. \& Ivry, R. Savings upon Re-Aiming in Visuomotor Adaptation. Journal of Neuroscience 35, 14386-14396 (2015).

23. Avraham, G., Keizman, M. \& Shmuelof, L. Environmental Consistency Modulation of Error Sensitivity During Motor Adaptation is Explicitly Controlled. Journal of Neurophysiology (2019) doi:10.1152/jn.00080.2019.

24. Avraham, G., Morehead, J. R., Kim, H. E. \& Ivry, R. B. Reexposure to a sensorimotor perturbation produces opposite effects on explicit and implicit learning processes. PLOS Biology 19, e3001147 (2021).

25. Yin, C. \& Wei, K. Savings in sensorimotor adaptation without an explicit strategy. Journal of Neurophysiology 123, 1180-1192 (2020).

26. Albert, S. T. et al. An implicit memory of errors limits human sensorimotor adaptation. Nature Human Behaviour (2021) doi:10.1038/s41562-020-01036-x. 
27. Smith, M. A., Ghazizadeh, A. \& Shadmehr, R. Interacting adaptive processes with different timescales underlie short-term motor learning. PLOS Biology 4, e179 (2006).

28. Albert, S. T. \& Shadmehr, R. Estimating properties of the fast and slow adaptive processes during sensorimotor adaptation. Journal of Neurophysiology 119, 1367-1393 (2018).

29. Thoroughman, K. \& Shadmehr, R. Learning of action through adaptive combination of motor primitives. Nature 407, 742-7 (2000).

30. Neville, K.-M. \& Cressman, E. K. The influence of awareness on explicit and implicit contributions to visuomotor adaptation over time. Experimental Brain Research 236, 2047-2059 (2018).

31. Benson, B. L., Anguera, J. A. \& Seidler, R. D. A spatial explicit strategy reduces error but interferes with sensorimotor adaptation. Journal of Neurophysiology 105, 2843-2851 (2011).

32. Fernandez-Ruiz, J., Wong, W., Armstrong, I. T. \& Flanagan, J. R. Relation between reaction time and reach errors during visuomotor adaptation. Behavioural Brain Research 219, 8-14 (2011).

33. Saijo, N. \& Gomi, H. Multiple Motor Learning Strategies in Visuomotor Rotation. PLOS ONE 5, e9399 (2010).

34. Bond, K. M. \& Taylor, J. A. Flexible explicit but rigid implicit learning in a visuomotor adaptation task. Journal of Neurophysiology 113, 3836-3849 (2015).

35. Tsay, J. S., Ivry, R. B., Lee, A. \& Avraham, G. Moving outside the lab: The viability of conducting sensorimotor learning studies online. Neurons, Behavior, Data analysis, and Theory 5, (2021).

36. Salomonczyk, D., Cressman, E. K. \& Henriques, D. Y. P. Proprioceptive recalibration following prolonged training and increasing distortions in visuomotor adaptation. Neuropsychologia 49, 3053-3062 (2011).

37. Taylor, J. A., Krakauer, J. W. \& Ivry, R. B. Explicit and Implicit Contributions to Learning in a Sensorimotor Adaptation Task. Journal of Neuroscience 34, 3023-3032 (2014).

38. Shadmehr, R., Brandt, J. \& Corkin, S. Time-dependent motor memory processes in amnesic subjects. Journal of Neurophysiology 80, 1590-1597 (1998).

39. Vaswani, P. A. et al. Persistent Residual Errors in Motor Adaptation Tasks: Reversion to Baseline and Exploratory Escape. Journal of Neuroscience 35, 6969-6977 (2015).

40. Kim, H. E., Morehead, J. R., Parvin, D. E., Moazzezi, R. \& Ivry, R. B. Invariant errors reveal limitations in motor correction rather than constraints on error sensitivity. Communications Biology 1, 19 (2018).

41. Maresch, J., Werner, S. \& Donchin, O. Methods matter: Your measures of explicit and implicit processes in visuomotor adaptation affect your results. European Journal of Neuroscience 53, 504-518 (2021). 
3724

3725

3726

3727

3728

3729

3730

3731

3732

3733

3734

3735

3736

3737

3738

3739

3740

3741

3742

3743

3744

3745

3746

3747

3748

3749

3750

3751

3752

3753

3754

3755

42. Kagerer, F. A., Contreras-Vidal, J. L. \& Stelmach, G. E. Adaptation to gradual as compared with sudden visuo-motor distortions. Experimental Brain Research 115, 557-561 (1997).

43. Bromberg, Z., Donchin, O. \& Haar, S. Eye Movements during Visuomotor Adaptation Represent Only Part of the Explicit Learning. eNeuro 6, ENEURO.0308-19.2019 (2019).

44. McDougle, S. D. \& Taylor, J. A. Dissociable cognitive strategies for sensorimotor learning. Nature Communications 10, 40 (2019).

45. Leow, L.-A., Gunn, R., Marinovic, W. \& Carroll, T. J. Estimating the implicit component of visuomotor rotation learning by constraining movement preparation time. Journal of Neurophysiology 118, 666-676 (2017).

46. Hwang, E. J. \& Shadmehr, R. Internal models of limb dynamics and the encoding of limb state. Journal of Neural Engineering 2, S266-78 (2005).

47. Krakauer, J. W., Pine, Z. M., Ghilardi, M. F. \& Ghez, C. Learning of visuomotor transformations for vectorial planning of reaching trajectories. Journal of Neuroscience 20, 8916-8924 (2000).

48. Fernandes, H. L., Stevenson, I. H. \& Kording, K. P. Generalization of stochastic visuomotor rotations. PLOS ONE 7, e43016 (2012).

49. Day, K. A., Roemmich, R. T., Taylor, J. A. \& Bastian, A. J. Visuomotor Learning Generalizes Around the Intended Movement. eNeuro 3, ENEURO.0005-16.2016 (2016).

50. McDougle, S. D., Bond, K. M. \& Taylor, J. A. Implications of plan-based generalization in sensorimotor adaptation. Journal of Neurophysiology 118, 383-393 (2017).

51. Herzfeld, D. J., Vaswani, P. A., Marko, M. K. \& Shadmehr, R. A memory of errors in sensorimotor learning. Science 345, 1349-1353 (2014).

52. Zarahn, E., Weston, G. D., Liang, J., Mazzoni, P. \& Krakauer, J. W. Explaining Savings for Visuomotor Adaptation: Linear Time-Invariant State-Space Models Are Not Sufficient. Journal of Neurophysiology 100, 2537-2548 (2008).

53. Huberdeau, D. M., Haith, A. M. \& Krakauer, J. W. Formation of a long-term memory for visuomotor adaptation following only a few trials of practice. Journal of Neurophysiology 114, 969-977 (2015).

54. Tsay, J. S., Haith, A. M., Ivry, R. B. \& Kim, H. E. Interactions between sensory prediction error and task error during implicit motor learning. bioRxiv 2021.06.20.449180 (2021) doi:10.1101/2021.06.20.449180.

55. Hwang, E. J., Smith, M. A. \& Shadmehr, R. Dissociable effects of the implicit and explicit memory systems on learning control of reaching. Experimental Brain Research 173, 425-437 (2006). 
56. Morehead, J. R. \& Orban de Xivry, J.-J. A Synthesis of the Many Errors and Learning Processes of Visuomotor Adaptation. bioRxiv 2021.03.14.435278 (2021) doi:10.1101/2021.03.14.435278.

57. Milner, B. Les troubles de la memoire accompagnant des lesions hippocampiques bilaterales. Physiologie de hippocampe (1962).

58. Gabrieli, J. D., Corkin, S., Mickel, S. F. \& Growdon, J. H. Intact acquisition and long-term retention of mirror-tracing skill in Alzheimer's disease and in global amnesia. Behavioral Neuroscience 107, 899-910 (1993).

59. Donchin, O. et al. Cerebellar regions involved in adaptation to force field and visuomotor perturbation. Journal of Neurophysiology 107, 134-147 (2012).

60. Smith, M. A. \& Shadmehr, R. Intact ability to learn internal models of arm dynamics in Huntington's disease but not cerebellar degeneration. Journal of Neurophysiology 93, 2809-2821 (2005).

61. Izawa, J., Criscimagna-Hemminger, S. E. \& Shadmehr, R. Cerebellar Contributions to Reach Adaptation and Learning Sensory Consequences of Action. Journal of Neuroscience 32, 42304239 (2012).

62. Wong, A. L., Marvel, C. L., Taylor, J. A. \& Krakauer, J. W. Can patients with cerebellar disease switch learning mechanisms to reduce their adaptation deficits? Brain 142, 662-673 (2019).

63. Becker, M. I. \& Person, A. L. Cerebellar Control of Reach Kinematics for Endpoint Precision. Neuron 103, 335-348.e5 (2019).

64. Morton, S. M. \& Bastian, A. J. Cerebellar contributions to locomotor adaptations during splitbelt treadmill walking. Journal of Neuroscience 26, 9107-9116 (2006).

65. Kording, K. P., Tenenbaum, J. B. \& Shadmehr, R. The dynamics of memory as a consequence of optimal adaptation to a changing body. Nature Neuroscience 10, 779-786 (2007).

66. MacLeod, C. M. Forgotten but Not Gone: Savings for Pictures and Words in Long-Term Memory. Journal of Experimental Psychology 14, 195-212 (1988).

67. Ebbinghaus, H. Uber das Gedachtnis. (Dunacker and Humblot, 1885).

68. Kitago, T., Ryan, S. L., Mazzoni, P., Krakauer, J. W. \& Haith, A. M. Unlearning versus savings in visuomotor adaptation: comparing effects of washout, passage of time, and removal of errors on motor memory. Frontiers in Human Neuroscience 7, 307 (2013).

69. Leow, L.-A., de Rugy, A., Marinovic, W., Riek, S. \& Carroll, T. J. Savings for visuomotor adaptation require prior history of error, not prior repetition of successful actions. Journal of Neurophysiology 116, 1603-1614 (2016). 
70. Wilterson, S. A. \& Taylor, J. A. Implicit Visuomotor Adaptation Remains Limited after Several Days of Training. eNeuro 8, ENEURO.0312-20.2021 (2021).

71. Miall, R. C., Jenkinson, N. \& Kulkarni, K. Adaptation to rotated visual feedback: a re-examination of motor interference. Experimental Brain Research 154, 201-210 (2004).

72. Hadjiosif, A. M. \& Smith, M. A. Savings is restricted to the temporally labile component of motor adaptation. in Translational and Computational Motor Control 1-2 (2015).

73. Alhussein, L., Hosseini, E. A., Nguyen, K. P., Smith, M. A. \& Joiner, W. M. Dissociating effects of error size, training duration, and amount of adaptation on the ability to retain motor memories. Journal of Neurophysiology 122, 2027-2042 (2019).

74. Hosseini, E. A., Nguyen, K. P. \& Joiner, W. M. The decay of motor adaptation to novel movement dynamics reveals an asymmetry in the stability of motion state-dependent learning. PLOS Computational Biology 13, e1005492 (2017).

75. Joiner, W. M., Sing, G. C. \& Smith, M. A. Temporal specificity of the initial adaptive response in motor adaptation. PLOS Computational Biology 13, e1005438 (2017).

76. Zhou, W., Fitzgerald, J., Colucci-Chang, K., Murthy, K. G. \& Joiner, W. M. The temporal stability of visuomotor adaptation generalization. Journal of Neurophysiology 118, 2435-2447 (2017).

77. de Brouwer, A. J., Albaghdadi, M., Flanagan, J. R. \& Gallivan, J. P. Using gaze behavior to parcellate the explicit and implicit contributions to visuomotor learning. Journal of Neurophysiology 120, 1602-1615 (2018).

78. Marko, M. K., Haith, A. M., Harran, M. D. \& Shadmehr, R. Sensitivity to prediction error in reach adaptation. Journal of Neurophysiology 108, 1752-1763 (2012).

79. Wei, K. \& Kording, K. Relevance of error: what drives motor adaptation? Journal of neurophysiology 101, 655-664 (2009).

80. Heald, J. B., Lengyel, M. \& Wolpert, D. M. Contextual inference underlies the learning of sensorimotor repertoires. Nature 600, 489-493 (2021).

81. Tsay, J. S., Kim, H. E., Parvin, D. E., Stover, A. R. \& Ivry, R. B. Individual differences in proprioception predict the extent of implicit sensorimotor adaptation. Journal of Neurophysiology (2021) doi:10.1152/jn.00585.2020.

82. Tsay, J. S., Kim, H. E., Haith, A. M. \& Ivry, R. B. Proprioceptive Re-alignment drives Implicit Sensorimotor Adaptation. bioRxiv 2021.12.21.473747 (2021) doi:10.1101/2021.12.21.473747.

83. Sedaghat-Nejad, E. \& Shadmehr, R. The cost of correcting for error during sensorimotor adaptation. Proceedings of the National Academy of Sciences 118, e2101717118 (2021).

84. Medina, J. F. Teaching the cerebellum about reward. Nature Neuroscience 22, 846-848 (2019). 
85. Wagner, M. J., Kim, T. H., Savall, J., Schnitzer, M. J. \& Luo, L. Cerebellar granule cells encode the expectation of reward. Nature 544, 96-100 (2017).

86. Heffley, W. et al. Coordinated cerebellar climbing fiber activity signals learned sensorimotor predictions. Nature Neuroscience 21, 1431-1441 (2018).

87. Kostadinov, D., Beau, M., Blanco-Pozo, M. \& Häusser, M. Predictive and reactive reward signals conveyed by climbing fiber inputs to cerebellar Purkinje cells. Nature Neuroscience 22, 950-962 (2019).

88. Tanaka, H., Sejnowski, T. J. \& Krakauer, J. W. Adaptation to Visuomotor Rotation Through Interaction Between Posterior Parietal and Motor Cortical Areas. Journal of Neurophysiology 102, 2921-2932 (2009).

89. Shadmehr, R., Smith, M. A. \& Krakauer, J. W. Error correction, sensory prediction, and adaptation in motor control. Annual Review of Neuroscience 33, 89-108 (2010).

90. Leow, L.-A., Marinovic, W., de Rugy, A. \& Carroll, T. J. Task errors contribute to implicit aftereffects in sensorimotor adaptation. European Journal of Neuroscience 48, 3397-3409 (2018).

91. Huang, V. S., Haith, A., Mazzoni, P. \& Krakauer, J. W. Rethinking motor learning and savings in adaptation paradigms: model-free memory for successful actions combines with internal models. Neuron 70, 787-801 (2011).

92. Hanajima, R. et al. Modulation of error-sensitivity during a prism adaptation task in people with cerebellar degeneration. Journal of Neurophysiology 114, 2460-2471 (2015).

93. Kojima, Y. \& Soetedjo, R. Elimination of the error signal in the superior colliculus impairs saccade motor learning. Proceedings of the National Academy of Sciences 115, E8987--E8995 (2018).

94. Bastian, A. J., Martin, T. A., Keating, J. G. \& Thach, W. T. Cerebellar ataxia: abnormal control of interaction torques across multiple joints. Journal of Neurophysiology 76, 492-509 (1996).

95. Herzfeld, D. J., Kojima, Y., Soetedjo, R. \& Shadmehr, R. Encoding of error and learning to correct that error by the Purkinje cells of the cerebellum. Nature Neuroscience 21, 736-743 (2018).

96. Herzfeld, D. J., Kojima, Y., Soetedjo, R. \& Shadmehr, R. Encoding of action by the Purkinje cells of the cerebellum. Nature 526, 439-442 (2015).

97. Maschke, M., Gomez, C. M., Ebner, T. J. \& Konczak, J. Hereditary cerebellar ataxia progressively impairs force adaptation during goal-directed arm movements. Journal of Neurophysiology $\mathbf{9 1}$ 230-238 (2004).

98. Martin, T. A., Keating, J. G., Goodkin, H. P., Bastian, A. J. \& Thach, W. T. Throwing while looking through prisms. I. Focal olivocerebellar lesions impair adaptation. Brain 119 ( Pt 4, 1183-1198 (1996). 
3854 